\title{
Increasing the Depth of Mass Spectrometry-based Structural Analysis of Protein complexes through the use of Multiple Cross-linkers
}

\author{
Yue-He Ding ${ }^{1,2}$, Sheng-Bo Fan ${ }^{3}$, Shuang Li ${ }^{1}$, Bo-Ya Feng ${ }^{4}$, Ning Gao, ${ }^{4}$ Keqiong Ye \\ ${ }^{1}$, Si-Min $\mathrm{He}^{3}$, Meng-Qiu Dong ${ }^{1,2}$ \\ ${ }^{1}$ National Institute of Biological Sciences, Beijing, Beijing 102206, China \\ ${ }^{2}$ Graduate Program in Chinese Academy of Medical Sciences and Peking Union Medical College, Beijing, China \\ ${ }^{3}$ Key Lab of Intelligent Information Processing of Chinese Academy of Sciences (CAS); Institute of Computing \\ Technology of CAS; University of CAS, China. \\ ${ }^{4}$ Ministry of Education Protein Science Laboratory, Center for Structural Biology, School of Life Sciences, and \\ Department of Chemistry, Tsinghua University, Beijing 100084, China
}

*To whom correspondence should be addressed. Email: dongmengqiu@nibs.ac.cn

Supplemental Text. Reagents. …………………….........................................................

Supplemental Text. Cross-linking of the 70S ribosome ....................................................................

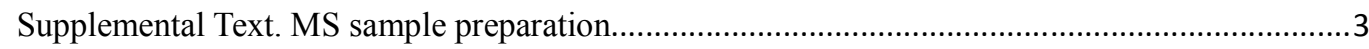

Supplemental Text. Mass spectrometry analysis............................................................................

Supplemental Text. Identification of cross-links using pLink .......................................................

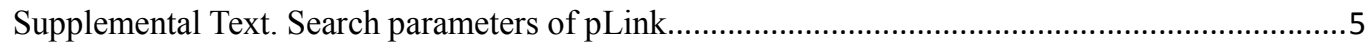

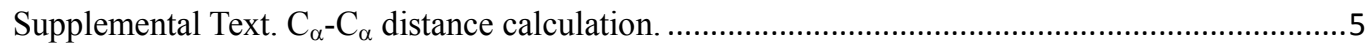

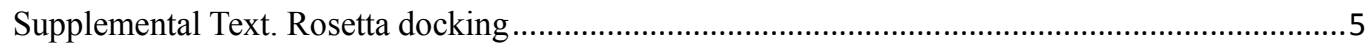

Supplemental Figure 1. Virtual cross-links of 1808 protein complexes. .................................

Supplemental Figure 2. Cross-linkers used in this study....................................................

Supplemental Figure 3. Experimental optimization of EDC/sulfo-NHS cross-linking. ................10

Supplemental Figure 4. Experimental optimization of amine-sulfhydryl cross-linkers................12

Supplemental Figure 5. Effects of TCEP and IAA treatment on amine-sulfhydryl cross-linking. .14

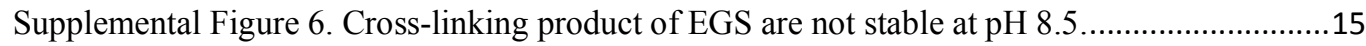

Supplemental Figure 7. $\mathrm{C}_{\alpha}-\mathrm{C}_{\alpha}$ distance distribution of the nine cross-linkers in the BSA, Aldolase,

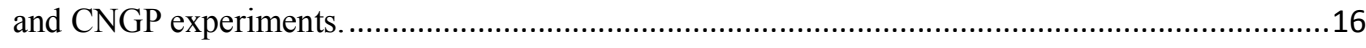

Supplemental Figure 8. Comparison of amine-amine or amine-sulfhydryl cross-linkers..............17 
Supplemental Figure 9. $\mathrm{C}_{\alpha}$ - $\mathrm{C}_{\alpha}$ distance distributions of the cross-linkers by molecular dynamic

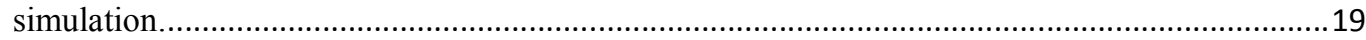

Supplemental Figure 10. Multiple cross-linkers provide complementary structural information...20

Supplemental Figure 11. Rosetta docking of Gar1 onto Cbf5-Nop10 .............................................21

Supplemental Figure 12. Rosetta docking of Nhp2 onto the CNG complex................................22

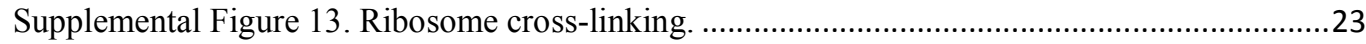

Supplemental Figure 14. Cross-links between RL1, RL7, RL31, and RS1 ...................................25

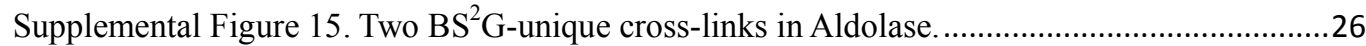

Supplemental Figure 16. Representative $\mathrm{MS}^{2}$ spectra of the nine cross-linkers..............................27

Supplemental Table 1. Cross-links identified in BSA, Aldolase, and the CNGP complex.............28

Supplemental Table 2. Cross-links identified in the 70S ribosome................................................55

Supplemental Table 3. Cross-links identified between Cbf5 and Gar1 1.........................................70

Supplemental Table 4. Cross-links identified between Nop10 and Nhp2 ......................................70

Supplemental Table 5. Clusters from docking of Gar1 onto Cbf5-Nop10 using Rosetta. ...............71

Supplemental Table 6. Clusters from docking of Nhp2onto Cbf5-Nop10-Gar1 using Rosetta......77 


\section{Supplemental Text. Reagents.}

All nine cross-linkers $\left(\mathrm{BS}^{2} \mathrm{G}, \mathrm{BS}^{3}\right.$, DSS, EGS, AMAS, GMBS, Sulfo-GMBS, EDC, Sulfo-NHS, and TFCS), as well as tris(2-carboxyethyl) phosphine (TCEP) and 2-Iodoacetamide (IAA), were purchased from Pierce biotechnology (Thermo Scientific). HEPES, DMSO, $\mathrm{NaCl}, \mathrm{KCl}, \mathrm{MgCl}_{2}$, Urea, $\mathrm{CaCl}_{2}$, Methylamine, Hydroxylamine $\cdot \mathrm{HCl}$, and Tris were purchased from Sigma. Acetonitrile (ACN), Formic acid (FA), Acetone, and $\mathrm{NH}_{4} \mathrm{HCO}_{3}$ were purchased from J. T. Baker. Mass-spectrometry-grade Trypsin was purchased from Promega.

GST was purified from E. coli and dissolved in $20 \mathrm{mM}$ HEPES, $150 \mathrm{mM} \mathrm{NaCl}$, pH 7.5 at 0.3 $\mathrm{mg} / \mathrm{ml}$. For BSA, lyophilized protein from Sigma was dissolved in $20 \mathrm{mM}$ HEPES, $150 \mathrm{mM} \mathrm{NaCl}$ at varying $\mathrm{pH}$ levels (as required). For Aldolase, an ammonium sulfate suspension from Sigma was dissovled in $20 \mathrm{mM}$ HEPES, $150 \mathrm{mM} \mathrm{NaCl}$ at the required $\mathrm{pH}$, and ammonium sulfate was removed using an Amicon filter. The CNGP complex was purified and assembled as previously described $^{1}$, and dissolved in $20 \mathrm{mM}$ HEPES, $500 \mathrm{mM} \mathrm{NaCl}$ at $\mathrm{pH}$ 8.0. $70 \mathrm{~S}$ ribosomes were purified as previously described ${ }^{2}$ and dissolved, at a final concentration of $1 \mu \mathrm{M}$, in $20 \mathrm{mM}$ HEPES, $100 \mathrm{mM} \mathrm{KCl}$, and $10 \mathrm{mM} \mathrm{MgCl}_{2}$ at $\mathrm{pH}$ 7.5.

\section{Supplemental Text. Cross-linking of the $70 \mathrm{~S}$ ribosome}

The crude $70 \mathrm{~S}$ ribosome was cross-linked by $\mathrm{BS}^{2} \mathrm{G}, \mathrm{BS}^{3}$, DSS, EGS, GMBS or Sulfo-GMBS at three different linker concentrations $(0.7 \mathrm{mM}, 1.5 \mathrm{mM}$, and $3 \mathrm{mM})$. For the EDC cross-linker reaction, three higher concentrations were used (3.0 mM EDC/4.5 mM Sulfo-NHS, $6.0 \mathrm{mM}$ EDC/9.0 mM Sulfo-NHS, and $12.0 \mathrm{mM} \mathrm{EDC/15.0} \mathrm{mM} \mathrm{Sulfo-NHS).} \mathrm{Reactions} \mathrm{were} \mathrm{quenched} \mathrm{as}$ described above.

\section{Supplemental Text. MS sample preparation}

Proteins were precipitated from the sample solution with a 4-fold volume of acetone for at least 30 minutes at $-20^{\circ} \mathrm{C}$. Pellets were air dried and resuspended in urea buffer $(8 \mathrm{M}$ urea, $100 \mathrm{mM}$ Tris, and $\mathrm{pH}$ 8.5). After reduction (5 $\mathrm{mM}$ TCEP, RT, $20 \mathrm{~min})$ and alkylation (10 $\mathrm{mM}$ iodoacetamide, RT, $15 \mathrm{~min})$, the samples were diluted with $2 \mathrm{M}$ urea with Tris buffer $(100 \mathrm{mM}, \mathrm{pH} 8.5)$. Denatured proteins were digested by trypsin $\left(1: 50\right.$ enzyme:substrate, $\mathrm{O} / \mathrm{N}$ at $\left.37^{\circ} \mathrm{C}\right)$, and the reactions were quenched with $5 \%$ formic acid (final conc.). TCEP and IAA treatments were not performed for amine-sulfhydryl cross-linkers. For EGS, Tris buffer at $\mathrm{pH} 7.0$ was used to prevent hydrolysis of EGS, and formic acid was supplied just prior to loading sample peptides for LC-MS/MS analysis (Supplemental Text).

\section{Supplemental Text. Mass spectrometry analysis}

The cross-linked HR-13 peptide was analyzed using LC-MS/MS on an Agilent 1200 LC system coupled to an LTQ Orbitrap-XL mass spectrometer (Thermo Fisher Scientific). To minimize variation, three linear peptides (DRVYIHPF, DRV $\mathrm{p}_{\mathrm{p}} \mathrm{YHHPF}$, and HSDAVFTDNYTR) were spiked in the samples to normalize each MS analysis. Peptides were loaded onto an analytical column (100 $\mu \mathrm{m}$ ID, packed with $8 \mathrm{~cm}$ Luna $\mathrm{C} 18$ resin ( $3 \mu \mathrm{m} 100 \AA$, Phenomenex)) using a pressure bomb. The peptides were separated using an acetonitrile gradient from $0-28 \%$ in $18 \mathrm{~min}$, and $28-$ 
$80 \%$ in an additional $2 \mathrm{~min}$ at a flow rate of $200 \mathrm{nl} / \mathrm{min}$. Spectra were acquired in data-dependent mode: the two most intense precursor ions from each full scan (resolution 60,000) were isolated for HCD MS2 (resolution 7,500, NCE 45) with a dynamic exclusion time of $5 \mathrm{~s}$. Precursors with $1+$ or unassigned charge states were rejected. Ion chromatographs were constructed using Xcalibur software, and the peak areas and intensities were normalized with those of the spiked peptides.

The LC-MS/MS analysis of BSA, Aldolase, and the CNGP complex was performed on an Accela Open AS (Thermo Fisher Scientific) coupled to an Orbitrap-Velos-Pro mass spectrometer (Thermo Fisher Scientific). Peptides were loaded onto an analytical column as noted above. The peptides were separated using an acetonitrile gradient from $0-28 \%$ in $45 \mathrm{~min}$ and $28-80 \%$ in and additional $5 \mathrm{~min}$ at a flow rate of $200 \mathrm{nl} / \mathrm{min}$. Spectra were acquired in data-dependent mode: the 10 most intense precursor ions from each full scan (resolution 60,000) were isolated for HCD MS2 (resolution 7,500, NCE 35) with a dynamic exclusion time of 30 s. Precursors with 1+, 2+, or unassigned charge states were rejected with the aim of increasing the $\mathrm{MS}^{2}$ spectral counts from cross-linked peptides.

The LC-MS/MS analysis of EGS cross-linking and all ribosome samples was performed on an Easy-nLC 1000 UHPLC (Thermo Fisher Scientific) coupled to a Q Exactive-Orbitrap mass spectrometer (Thermo Fisher Scientific). Peptides were loaded on a pre-column (75 $\mu \mathrm{m} \mathrm{ID,} 8 \mathrm{~cm}$ long, packed with ODS-AQ $12 \mathrm{~nm}-10 \mu \mathrm{m}$ beads from YMC Co., Ltd.) and separated on an analytical column (75 mm ID, $11 \mathrm{~cm}$ long, packed with Luna C18 $1.8 \mathrm{~mm} 100 \AA$ resin from Welch Materials) using an acetonitrile gradient from $0-25 \%$ in $55 \mathrm{~min}$ ( $83 \mathrm{~min}$ for the ribosome samples) at a flow rate of $200 \mathrm{nl} / \mathrm{min}$. The top 10 most abundant precursor ions from each full scan (resolution 70,000) were isolated for HCD MS2 (resolution 17,500; NCE 27), with a dynamic exclusion time of $30 \mathrm{~s}$. Precursors with unassigned charge states or charge states of $1+, 2+$, or $>6+$, were excluded.

\section{Supplemental Text. Identification of cross-links using pLink}

The search parameters used for pLink were similar to those previously described elsewhere ${ }^{3}$ and the details are in the Supporting Text blow. The protein sequences of BSA, Aldolase, or CNGP were used for database searching, as appropriate. For the ribosome sample, a conventional protein identification was performed using Prolucid against an E. coli database from UniProt-Swissprot, and a specified database was generated for pLink searching ${ }^{3}$. $\mathrm{MS}^{2}$ spectra were labeled with pLabel $^{3}$, requiring 20 p.p.m. mass deviations. For the BSA, Aldolase, and CNGP data, the results were filtered with the following criteria: FDR $<5 \%$, E-Value $<0.01$, each cross-link must be identified in at least two of the four replicates and have a total spectral count of four or more. For the $70 \mathrm{~S}$ ribosome data, the results were filtered by requiring FDR $<5 \%$, E-Value $<0.01$ and at least two spectra among six replicates (two technical repeats for each cross-linker concentration). Representative spectra for each cross-linker are shown in Supplemental Figure 16. 


\section{Supplemental Text. Search parameters of pLink}

The search parameters used for pLink were similar to those previously described elsewhere ${ }^{3}$ : instrument $\mathrm{HCD}$, precursor mass tolerance 20 p.p.m., fragment mass tolerance 20 p.p.m., cross-linker $\mathrm{BS}^{2} \mathrm{G}$ (cross-linking sites $\mathrm{K}$ and protein $\mathrm{N}$ terminus, cross-link mass-shift 96.0211296, mono-link mass-shift 114.0316942), $\mathrm{BS}^{3} / \mathrm{DSS}$ (cross-linking sites $\mathrm{K}$ and protein $\mathrm{N}$ terminus, cross-link mass-shift 138.0680796, mono-link mass-shift 156.0786442), EGS (cross-linking sites $\mathrm{K}$ and protein $\mathrm{N}$ terminus, cross-link mass-shift 226.04774, mono-link mass-shift 244.0583), AMAS (cross-linking sites K or protein $\mathrm{N}$ terminus with Cysteine, cross-link mass-shift 137.011, mono-link mass-shift 155.02156), GMBS/Sulfo-GMBS (cross-linking sites $\mathrm{K}$ or protein $\mathrm{N}$ terminus with cysteine, cross-link mass-shift 165.0422, mono-link mass-shift 183.05276), EDC (cross-linking sites $\mathrm{K}$ or protein $\mathrm{N}$ terminus with $\mathrm{D}$ or E, cross-link mass-shift -18.01056), TFCS (cross-linking sites $\mathrm{K}$ or protein $\mathrm{N}$ terminus with $\mathrm{D}$ or $\mathrm{E}$, cross-link mass-shift 95.0735), for non-sulfhydryl cross-linker fixed modification C 57.02146, peptide length minimum 4 amino acids and maximum 100 amino acids per chain, peptide mass minimum 400 and maximum 10,000 Da per chain, enzyme Trypsin, two missed cleavage sites per chain (four per cross-link).

\section{Supplemental Text. $\mathrm{C}_{\alpha}-\mathrm{C}_{\alpha}$ distance calculation.}

The $\mathrm{C}_{\alpha}-\mathrm{C}_{\alpha}$ Euclidean distances were calculated with an in-house Perl script with the coordinates from the PDB files. For BSA and Aldolase, the status of a cross-link (either intra- or inter-molecular) could not be determined based on the sequences of the cross-linked peptides. We thus calculated all of the possible combinations and picked the ones with the shortest $\mathrm{C}_{\alpha}-\mathrm{C}_{\alpha}$ distance. When calculating structure compatibility, the distance cutoffs are: $20.3 \AA$ for $B S^{2} \mathrm{G}, 24.0$ $\AA$ for $\mathrm{BS}^{3} / \mathrm{DSS}, 28.7 \AA$ for EGS, $11.2 \AA$ for EDC, $20.5 \AA$ for TFCS, $16.0 \AA$ for AMAS and $18.2 \AA$ for GMBS/Sulfo-GMBS.

\section{Supplemental Text. Rosetta docking}

Rosetta Version 3.5 was used to do the protein-protein docking simulations. The structures of the docking partners from PDB were prepacked using the docking prepack protocol, and the smaller partner was treated as the ligand. In low resolution docking, 100,000 (for Gar1 to Cbf5-Nop10) or 200,000 (for Nhp2 to Cbf5-Nop10-Gar1) models were generated. To save CPU time, only $\mathrm{C}_{\alpha}$ was considered for ligand RMSD (L-RMSD) calculations in this study. The 200 models with the lowest energy were clustered using an R script, as described previously ${ }^{4}$. Hierarchical cluster trees were cut at an L-RMSD threshold of $20 \AA$. The best model of each cluster was refined with the Rosetta local refinement protocol, and the pose with lowest energy was chosen as the representative model. Rosetta filtering was turned off to save CPU time, but additional filtering was performed optionally with an in-house Perl script which required that the models satisfy all constraints given. Structures were illustrated using PyMOL ${ }^{5}$. 
The CXMS constraints were given in the following format: AtomPair \{atom_name1\} \{residue_number1Chain_ID1\} \{atom_name2\} \{residue_number2Chain_ID2\} BOUNDED $\{\mathrm{lb}\}$ $\{\mathrm{ub}\}\{\mathrm{sd}\}\{$ rswitch $\{\operatorname{tag}\}$. The parameters were $l b=0, u b=30, s d=1$ and $r$ switch $=0.5$. The BOUNDED function is shown below.

$$
f(x)=\left\{\begin{array}{r}
0 \text { for } 0 \leq x \leq u b \\
\left(\frac{x-u b}{s d}\right)^{2} \text { for } u b<x \leq u b+\text { rswitch } \cdot s d \\
\frac{1}{s d}(x-(u b+\text { rswitch } \cdot s d))+\left(\frac{r s w i t c h \cdot s d}{s d}\right)^{2} \text { for } x>u b+\text { rswitch } \cdot \text { sd }
\end{array}\right.
$$

Flags for Rosetta docking are shown below.

Docking prepack flags:

-s $\{$ protein.pdb\}

$-\mathrm{ex} 1$

-ex2aro

-use_input_sc

-docking_local_refine

-out:overwrite

-out:file:fullatom

-mute core.io.database

Low resolution docking flags:

-s \{protein.pdb\}

-partners \{docking partners\}

-low_res_protocol_only

-randomize1

-randomize2

-spin

-no_filters

-constraints:cst_file \{constrain_file\}

-out:nstruct $\{100000$ or 200000$\}$

-out:overwrite

Local refine flags:

-s \{protein.pdb\}

-partners \{docking_partners\}

-ex1

-ex2aro

-dock_pert 38

-native \{native_structure.pdb

-norepack1

-docking:sc_min

-dock_rtmin

-out:overwrite

-out:file:fullatom

-mute core.io.database 
-out:nstruct $\{1000\}$ 


\section{Supplemental Figure 1. Virtual cross-links of 1808 protein complexes.}

Distribution of vXLs using amine-amine, amine-sulfhydryl, and amine-carboxyl cross-linkers, and their combinations, with a $22 \AA \mathrm{C}_{\beta}-\mathrm{C}_{\beta}$ distance cutoff $\left(\sim 24 \AA\right.$ for $\mathrm{C}_{\alpha}-\mathrm{C}_{\alpha}$ distance like $\left.\mathrm{BS}^{3} / \mathrm{DSS}\right)$. Details for complexes with more than five vXLs are shown in $\mathbf{b}$.

\section{Supplemental Figure 1}

a

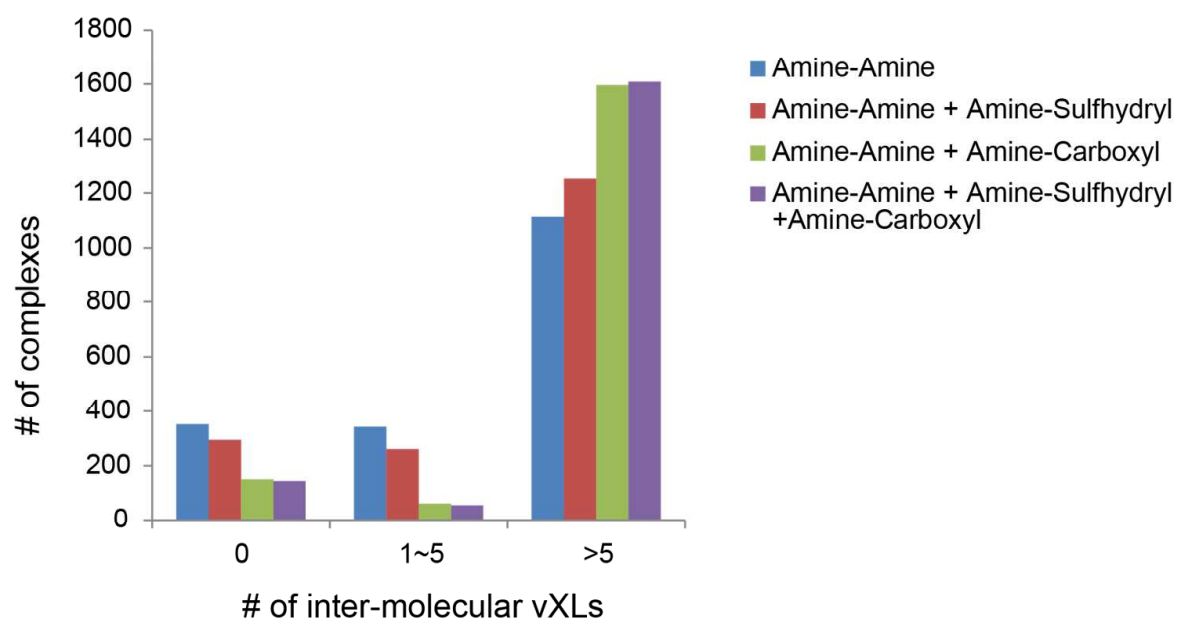

b

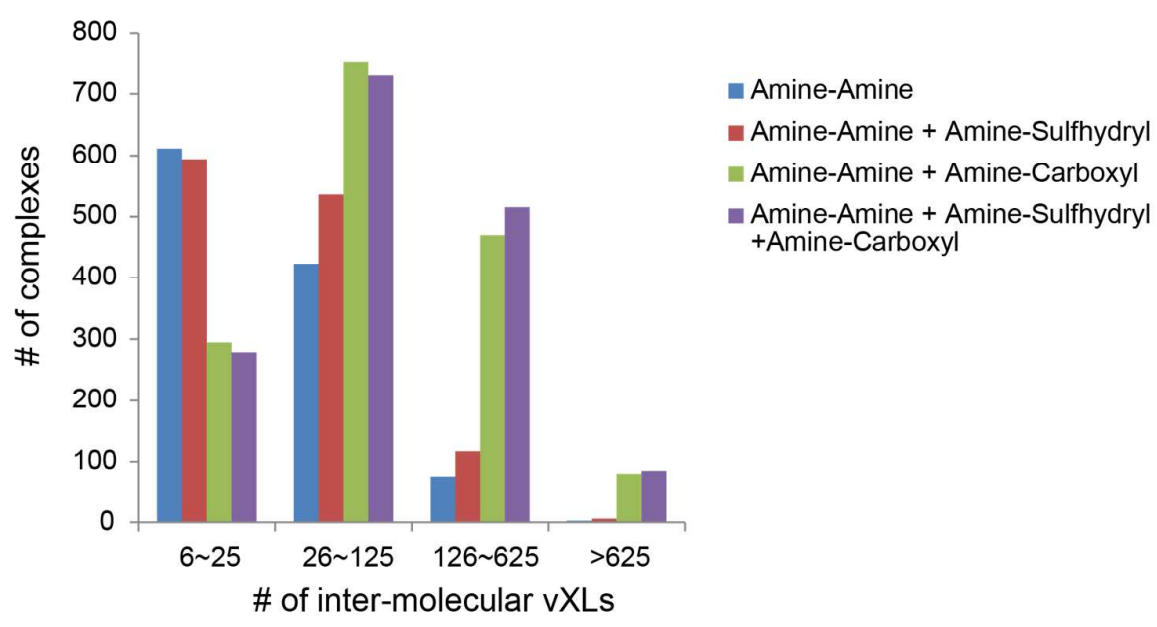




\section{Supplemental Figure 2. Cross-linkers used in this study.}

Nine cross-linkers, including amine-amine (BS ${ }^{2} \mathrm{G}, \mathrm{BS}^{3}$, DSS and EGS), amine-sulfhydryl (AMAS, GMBS and Sulfo-GMBS), and amine-carboxyl (EDC and TFCS) cross-linkers were evaluated in this study. The spacer arm lengths and theoretical $\mathrm{C}_{\alpha}-\mathrm{C}_{\alpha}$ distances are shown under the corresponding chemical structures.

Supplemental Figure 2

$-\mathrm{NH}_{2} \&-\mathrm{NH}_{2}$

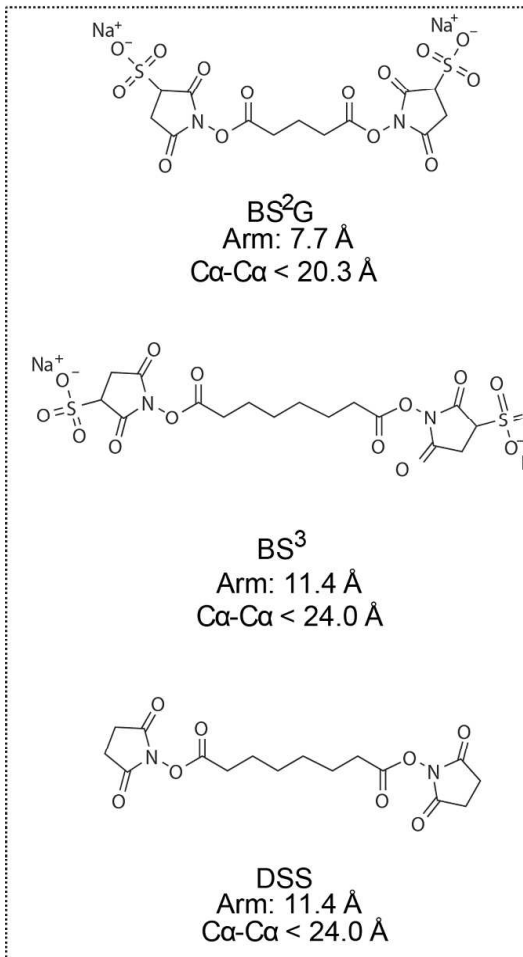

$-\mathrm{NH}_{2} \&-\mathrm{COOH}$

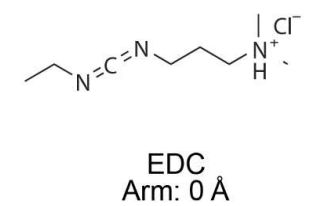

$\mathrm{C} \alpha-\mathrm{C} \alpha<9.9 \AA(\mathrm{K}-\mathrm{D})$ or $11.2 \AA(\mathrm{K}-\mathrm{E})$

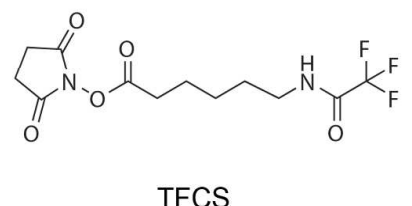

Arm: $9.3 A$

$\mathrm{C} \alpha-\mathrm{C} \alpha<19.2 \AA(\mathrm{K}-\mathrm{D})$ or $20.5 \AA(\mathrm{K}-\mathrm{E})$
$-\mathrm{NH}_{2} \&-\mathrm{SH}$

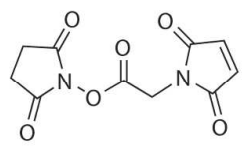

AMAS

$\mathrm{Ca}-\mathrm{Ca}<16.0 \AA$

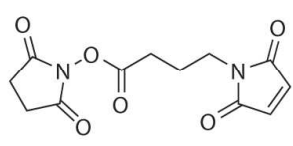

GMBS

Arm: $9.1 \AA$

$\mathrm{Ca}-\mathrm{Ca}<18.2 \AA$

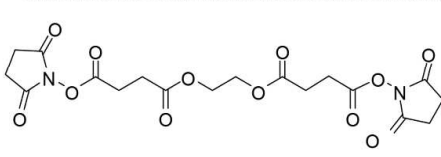

$\underbrace{O}_{0}$

Sulfo-GMBS

Arm: $9.1 \AA$

$\mathrm{C} \alpha-\mathrm{C} \alpha<18.2 \AA$ 


\section{Supplemental Figure 3. Experimental optimization of EDC/sulfo-NHS cross-linking.}

(a) Scheme of EDC/Sulfo-NHS cross-linking. The scheme is from the commercial EDC product instructions (Thermo Scientific). EDC was first reacted with carboxylic acid to form an unstable o-Acylisourea Intermediate that can produce stable amide bonds with primary amines or can undergo hydrolysis and release the carboxylic acid. Sulfo-NHS transforms the o-Acylisourea Intermediate to an amine-reactive Sulfo-NHS ester that is more stable and capable of reacting with primary amines to form an amide bond. (b) EDC cross-linking with or without Sulfo-NHS under different $\mathrm{pH}$ conditions were evaluated using SDS-PAGE, and the identification results are shown in (c). Concentrations of EDC and TFCS tested by both SDS-PAGE (d-e) and MS identification (f-g). The optimized conditions are labeled with red boxes. 
Supplemental Figure 3<smiles>[R]C(=O)O</smiles>

Carboxylic Acid<smiles>CCN=C=NCCC[N+](C)(C)[O-]</smiles>

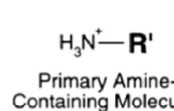<smiles>NS(=O)(=O)O[Mg]C1CC(=O)N(O)C1=O</smiles><smiles>[R]C(=O)OC(=NCCCNC)NCC</smiles>

Sulfo-NHS<smiles></smiles><smiles>C1CCCCC1</smiles><smiles>C1CCCCC1</smiles>
Primary AmineAmide Bond
Formation Active

b

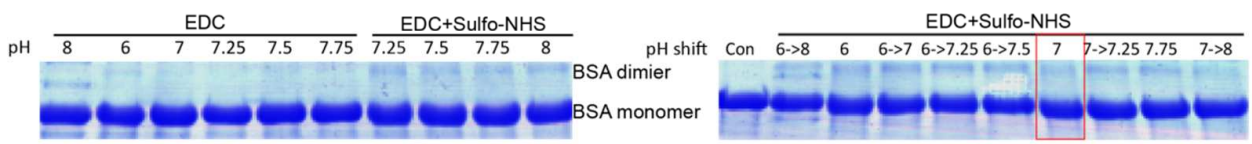

C

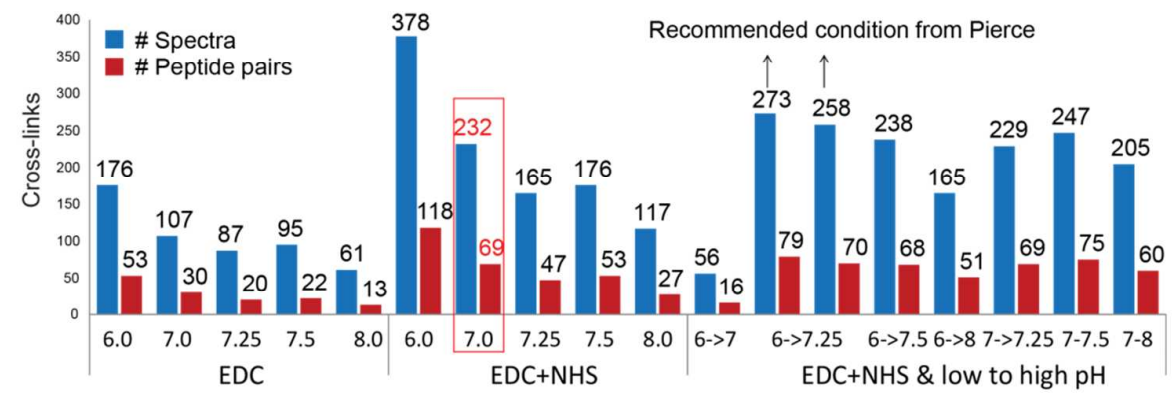

d

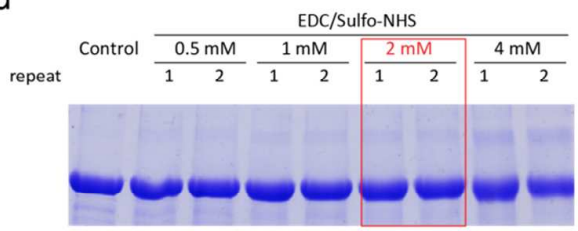

f

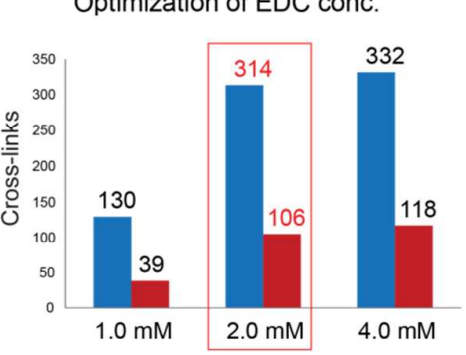

e

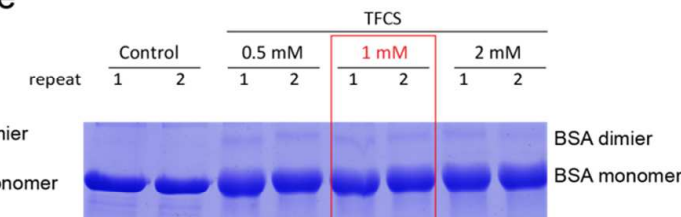

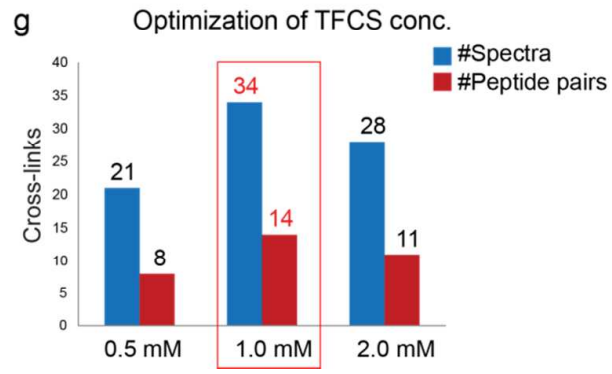




\section{Supplemental Figure 4. Experimental optimization of amine-sulfhydryl}

cross-linkers.

(a) Estimating the cross-linking products of BSA with AMAS, GMBS, and Sulfo-GMBS using SDS-PAGE. (b) Intensities of cross-linking products from a synthesized peptide were measured with LC-MS/MS. (c) SDS-PAGE showing the cross-linking products of GST with AMAS or GMBS with a reaction time from 10 minutes to 4 hours. (d) SDS-PAGE showing the BSA dimer with different concentrations of AMAS, GMBS, and Sulfo-GMBS. (e) The numbers of cross-linking site pairs of BSA identified with different concentrations of AMAS, GMBS and Sulfo-GMBS are shown in the bar graphs. The optimized conditions are labeled with red boxes. 
Supplemental Figure 4

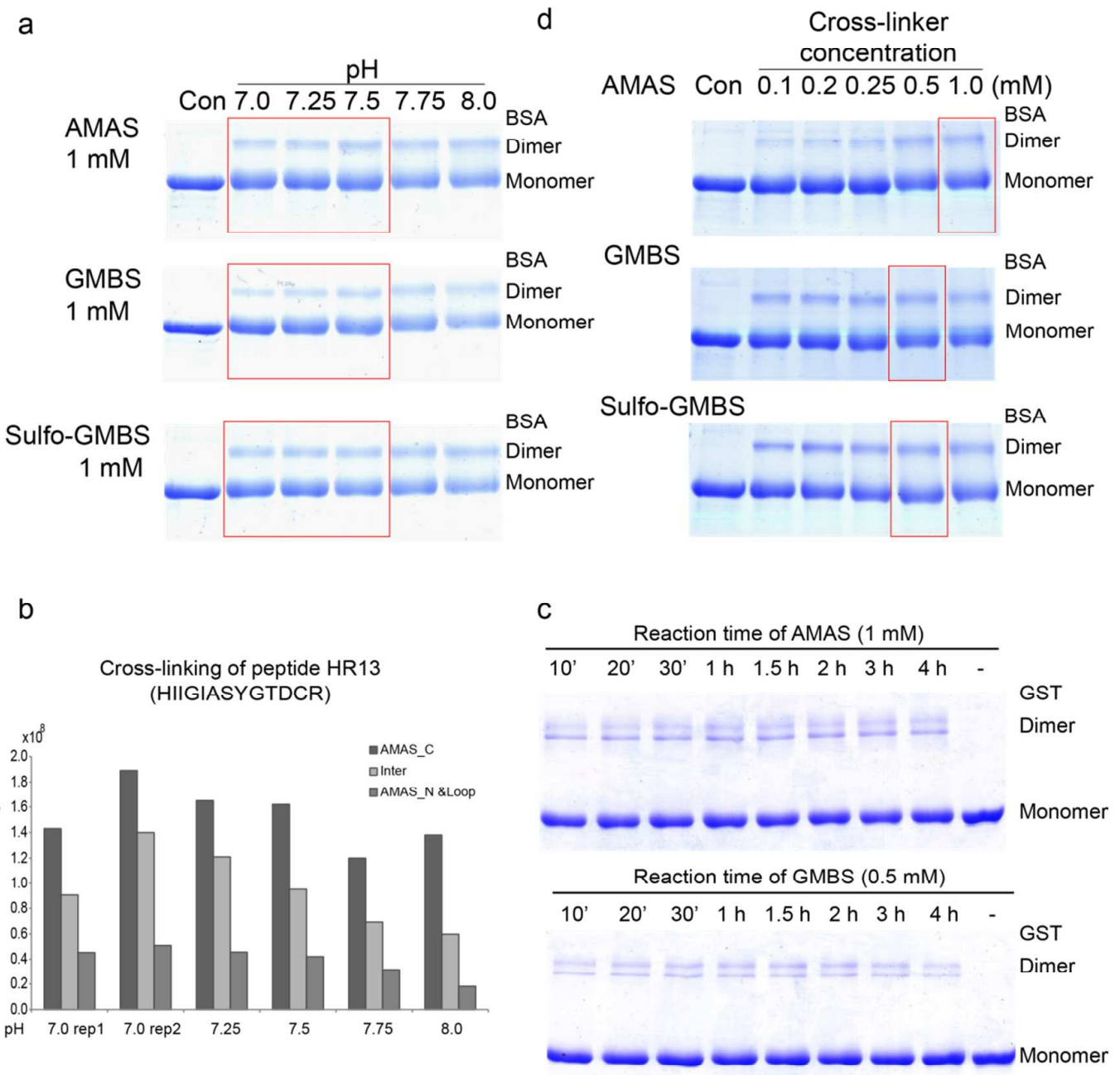

e
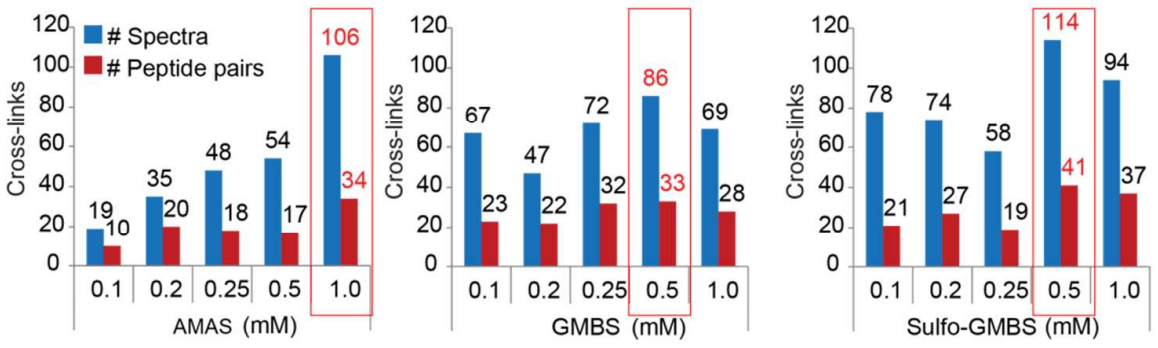


\section{Supplemental Figure 5. Effects of TCEP and IAA treatment on amine-sulfhydryl cross-linking.}

(a) LC-MS chromatographs of AMAS mono-link to the N-terminus $(\mathrm{m} / \mathrm{z} 771.85$, charge $2+)$ or to cysteine (m/z 780.86, charge 2+), loop-linked (m/z 771.85, charge $2+)$, and Inter-linked $(\mathrm{m} / \mathrm{z}$ 737.60 , charge $4+$ ) peptides were reconstructed using Xcalibur. The peak intensities are labeled. (b) The peak area of these peptides were calculated using Xcalibur, as shown in the bar graph. (c) The number of cross-linked site pairs from the CNGP complex identified with AMAS, GMBS, and Sulfo-GMBS treated with or without TCEP and IAA, shown as bar graphs. (d) The number of cross-linked peptide pairs of Aldolase identified with Sulfo-GMBS treated with or without $\beta M E$. The percentage represents how many cross-links were structurally compatible.

Supplemental Figure 5

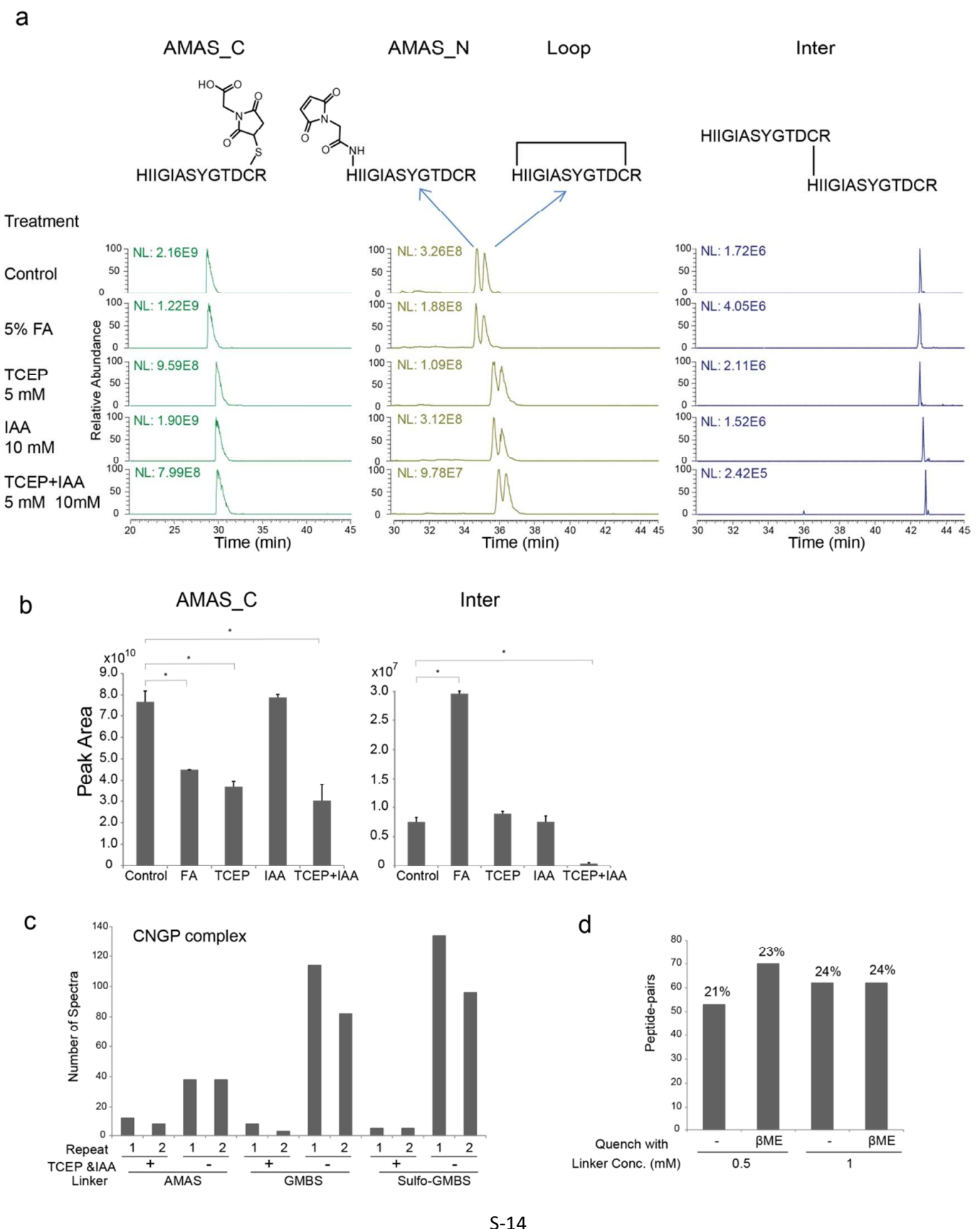




\section{Supplemental Figure 6. Cross-linking product of EGS are not stable at pH 8.5.}

In addition to mono-linked (adduction mass: 244.06), loop-linked, Inter-linked, and regular peptides, there are two hydrolyzed forms of mono-linked peptides (adduction mass: 100.01 and 144.04). The spectral counts of each form of cross-linking product are presented in the bar graph.

\section{Supplemental Figure 6}

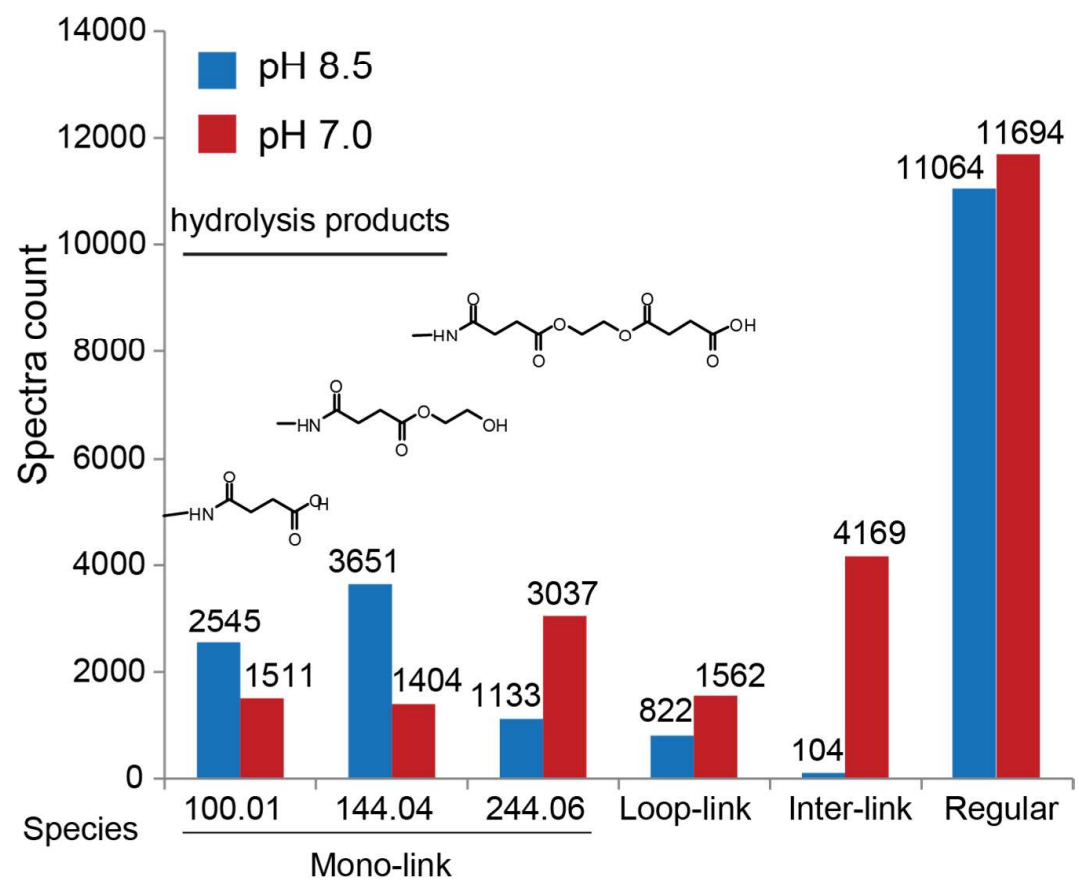




\section{Supplemental Figure 7. $\mathrm{C}_{\alpha}-\mathrm{C}_{\alpha}$ distance distribution of the nine cross-linkers in the BSA, Aldolase, and CNGP experiments.}

The cross-links of BSA, Aldolase, and CNGP were combined, and the distributions of $\mathrm{C}_{\alpha}-\mathrm{C}_{\alpha}$ distances were plotted with a bin size of $5 \AA$. The green lines indicate a distance of $24 \AA$, and the red lines show the theoretical maximum $\mathrm{C}_{\alpha}-\mathrm{C}_{\alpha}$ distance of the corresponding cross-linker.

Supplemental Figure 7
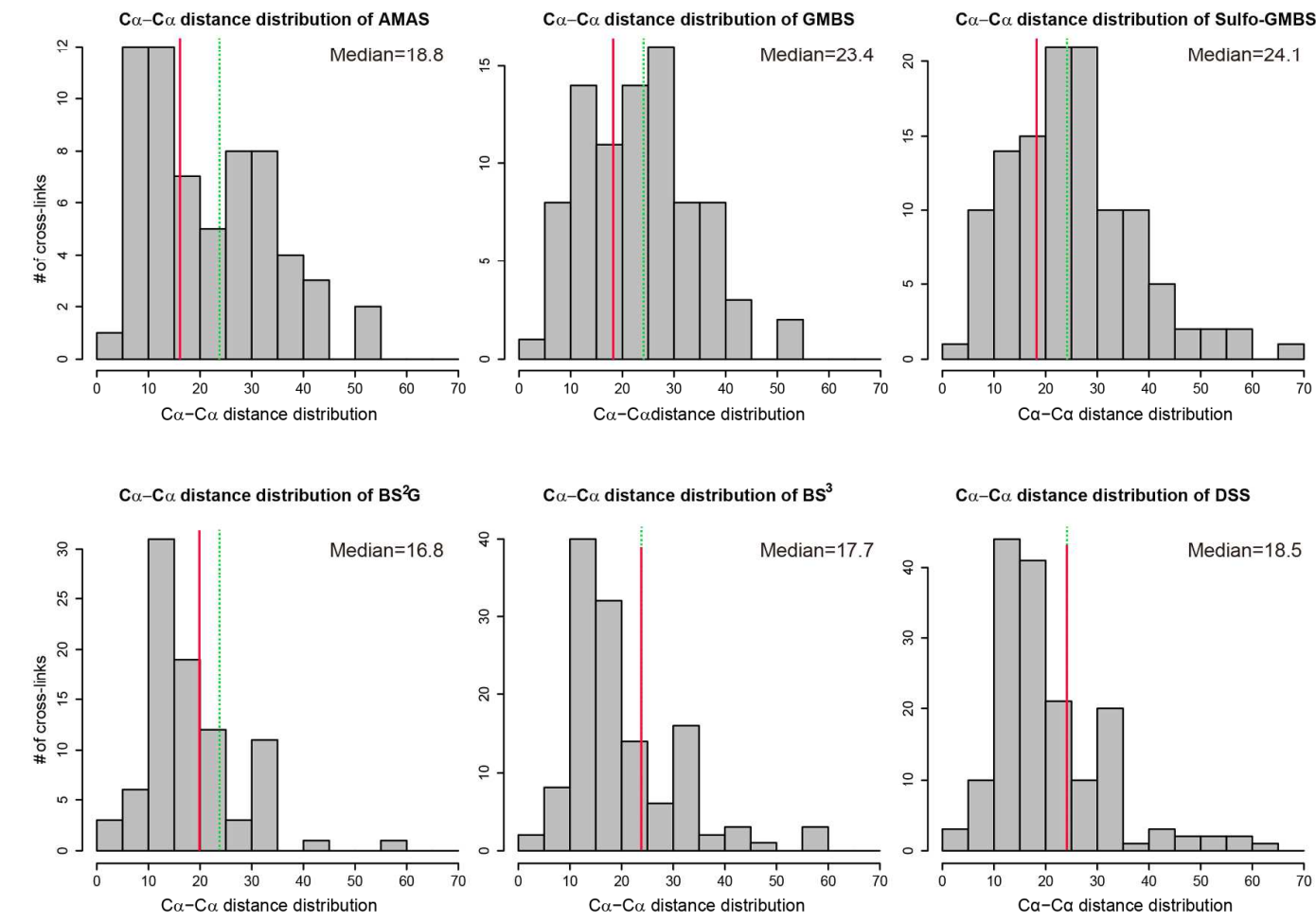

$\mathrm{C}_{\alpha}-\mathrm{C} \alpha$ distance distribution of $\mathrm{BS}^{3}$

$\mathrm{C}_{\alpha}-\mathrm{C}_{\alpha}$ distance distribution of DSS
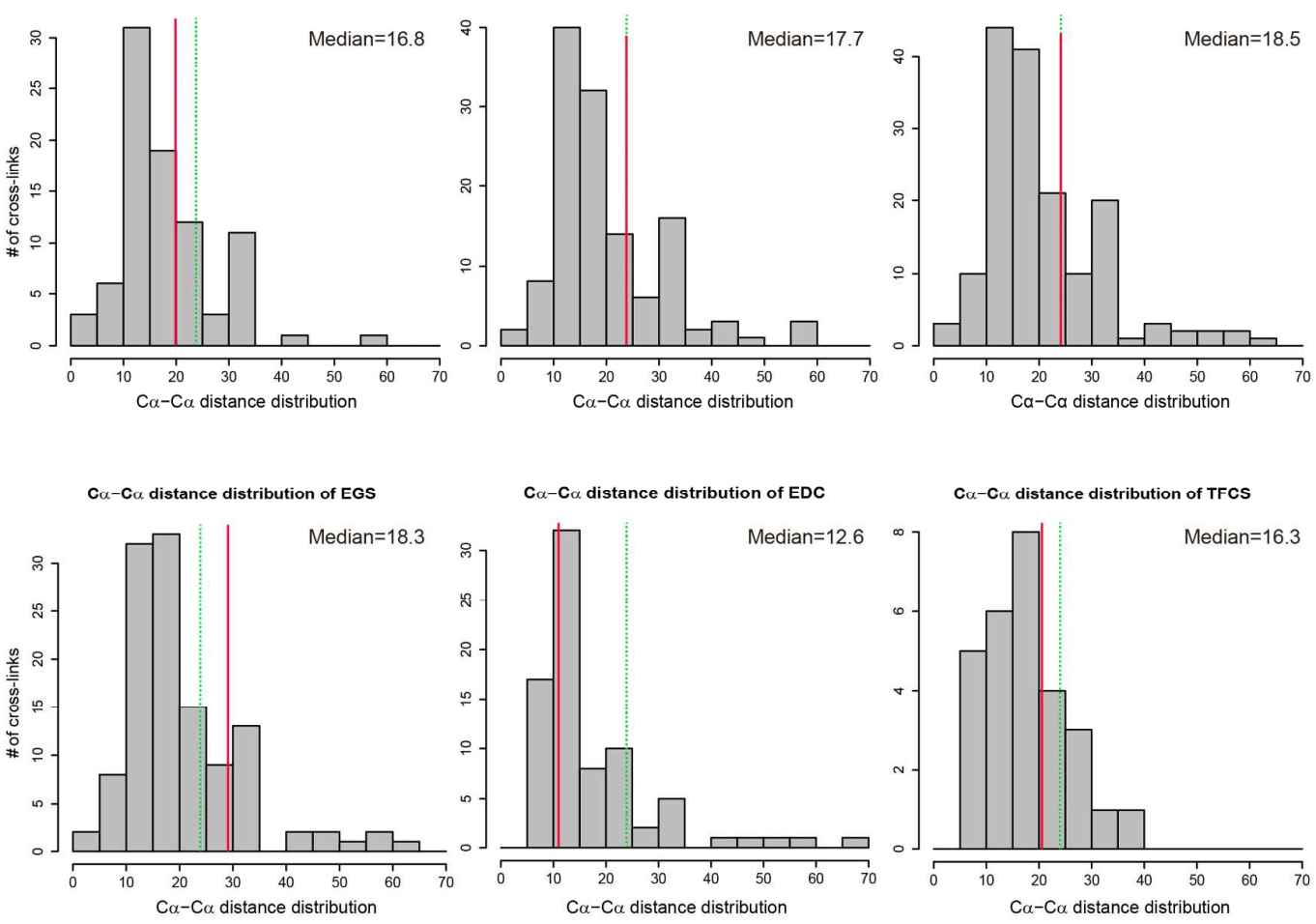

$\mathrm{C} u-\mathrm{C} u$ distance distribution of TFCS

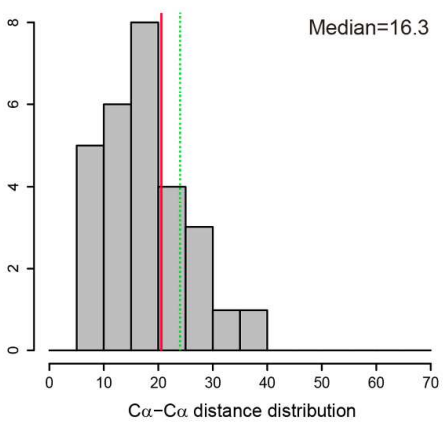


Supplemental Figure 8. Comparison of amine-amine or amine-sulfhydryl cross-linkers.

(a) Venn diagram comparing cross-links of BSA, Aldolase, and the CNG complex identified with amine-amine cross-linkers or amine-sulfhydryl cross-linkers. (b) Accumulation curves of identified non-redundant cross-links. Data from BSA, Aldolase, and CNGP are merged in the "Total" panel. 
Supplemental Figure 8

a

Amine-Amine cross-link
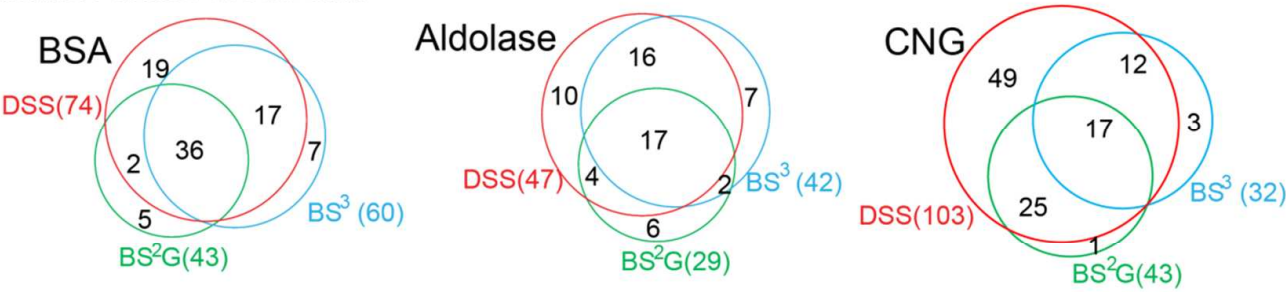

Amine-Sulfhydryl cross-link
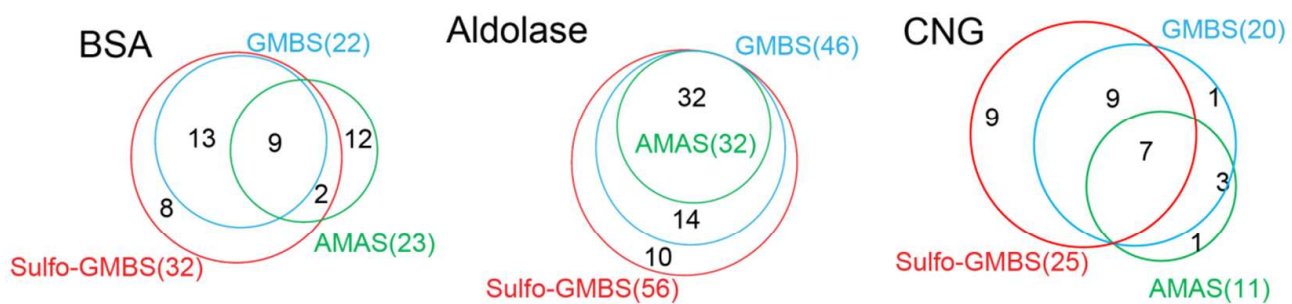

b
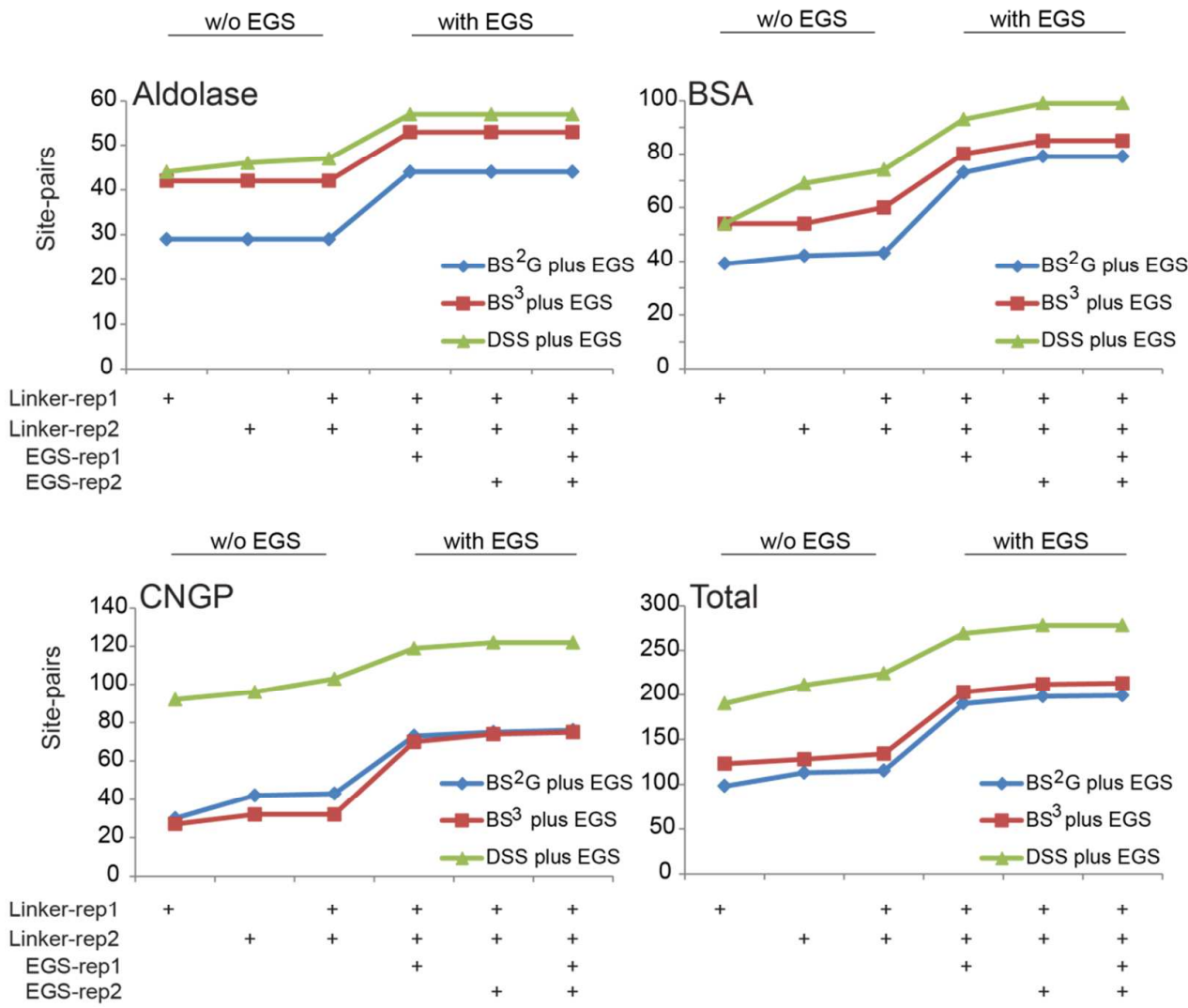
Supplemental Figure 9. $\mathrm{C}_{\alpha}-\mathrm{C}_{\alpha}$ distance distributions of the cross-linkers by molecular dynamic simulation.

Supplemental Figure 9
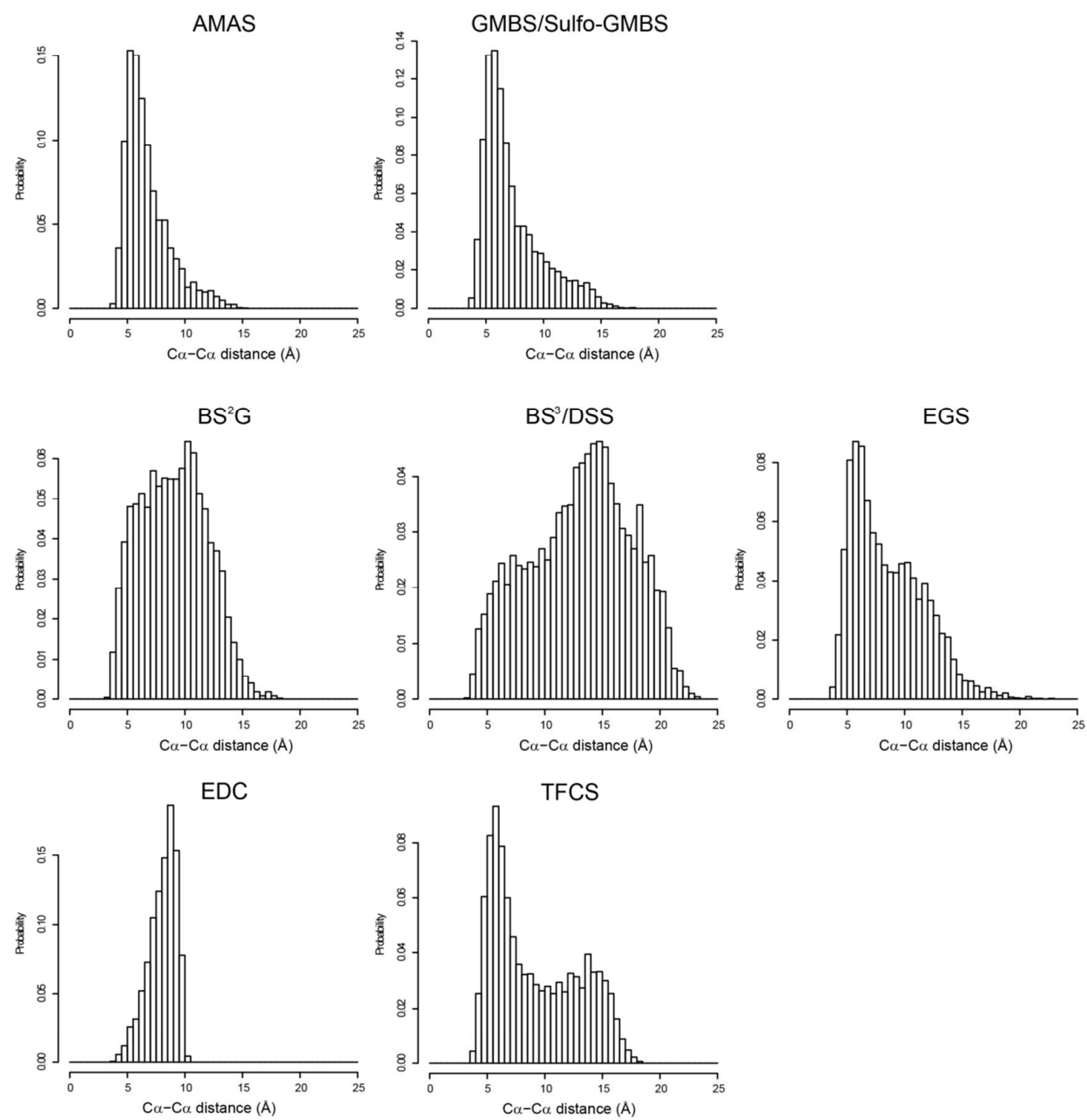


\section{Supplemental Figure 10. Multiple cross-linkers provide complementary structural information.}

(a) Intra-molecular cross-links of BSA (PDB: $3 \mathrm{~V} 03^{6}$ ). (b) Intra-molecular cross-links of Aldolase (PDB: 1ZAH). (c) Inter-molecular cross-links of the Aldolase homotetramer (PDB: 1ZAH ${ }^{7}$ ), with each chain in a different color. Amine-amine cross-links are colored blue, amine-sulfhydryl red, and amine-carboxyl magenta.

Supplemental Figure 10

a
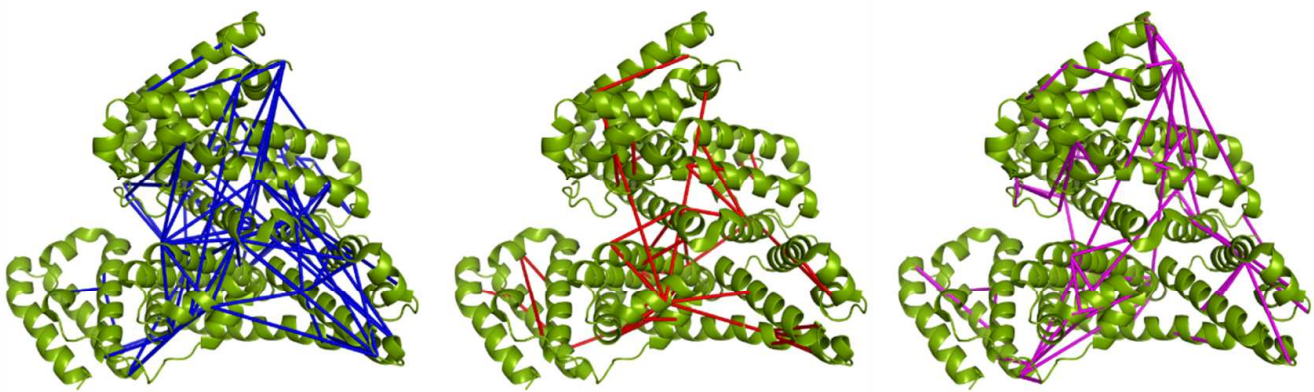

K(or N-term)-K(or N-term)

K(or N-term)-C

K(or N-term)-D/E

b
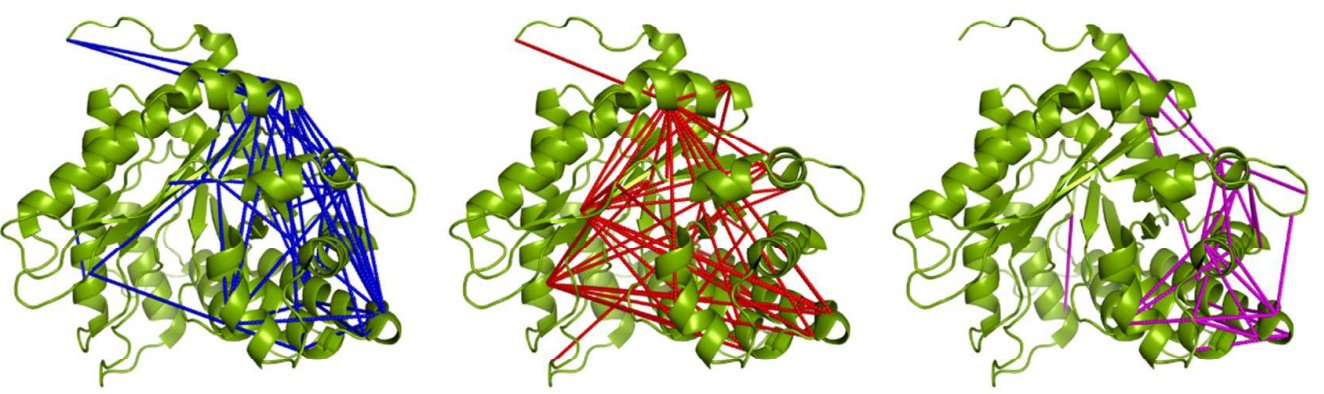

C

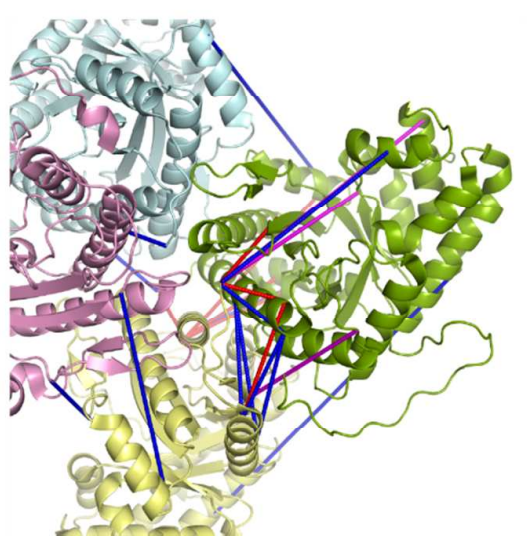




\section{Supplemental Figure 11. Rosetta docking of Gar1 onto Cbf5-Nop10.}

Cluster size and L-RMSD to the native structure are plotted for (a) low resolution docking, (b) low resolution docking with an additional filter, (c) local refinement of the representative model from (a), and (d) local refinement of the representative models from (b). Cluster numbers and average cluster size with different numbers of constraints applied are shown in (e) and (f), respectively.

Supplemental Figure 11

a
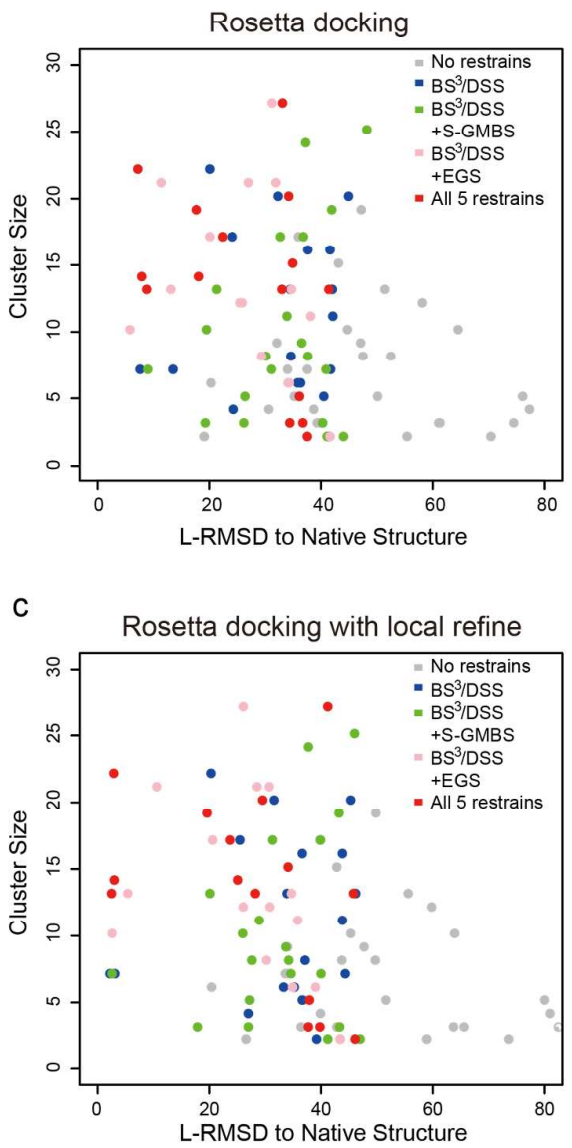

e

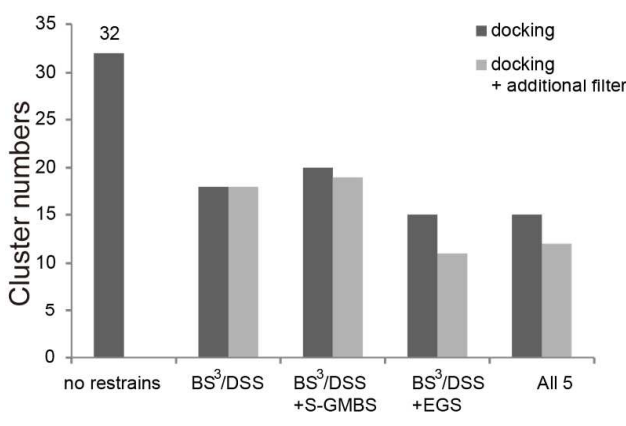

b

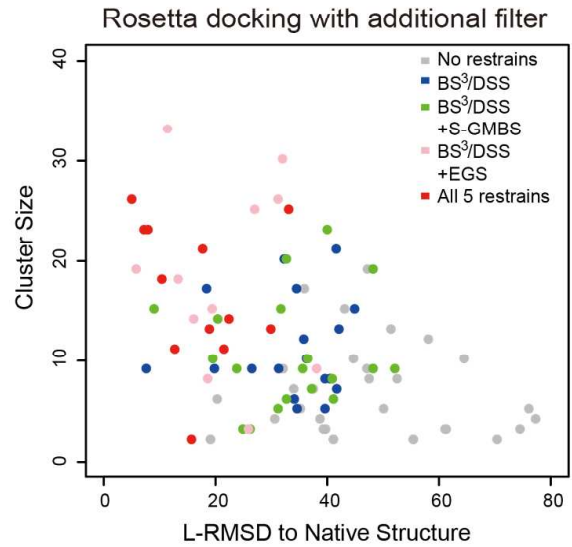

d Rosetta docking with additional filter and local refine

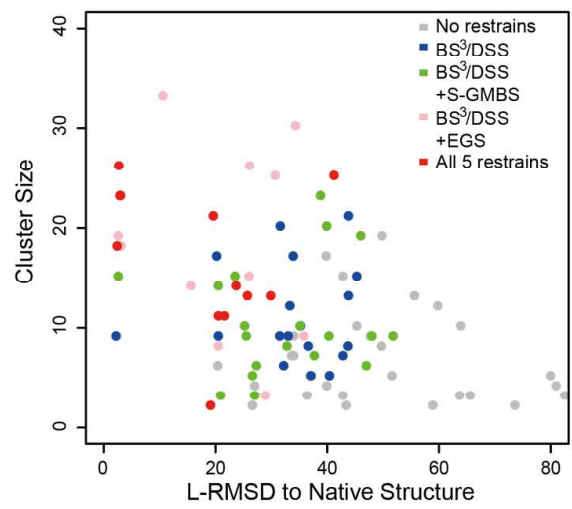

f

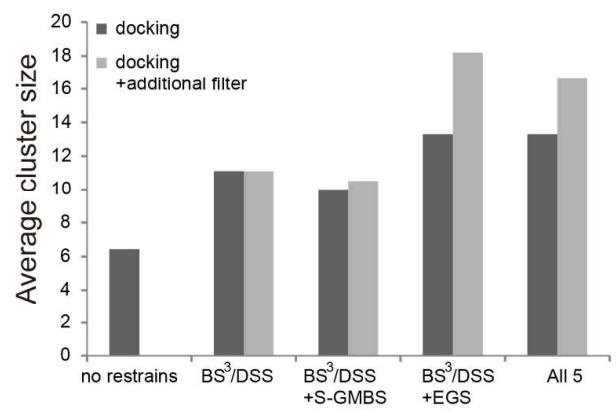




\section{Supplemental Figure 12. Rosetta docking of Nhp2 onto the CNG complex.}

(a) Cluster size and L-RMSD to the homology model are plotted. (b) L-RMSD distances between the final four clusters and the homology model are shown in tree view.

Supplemental Figure 12

a

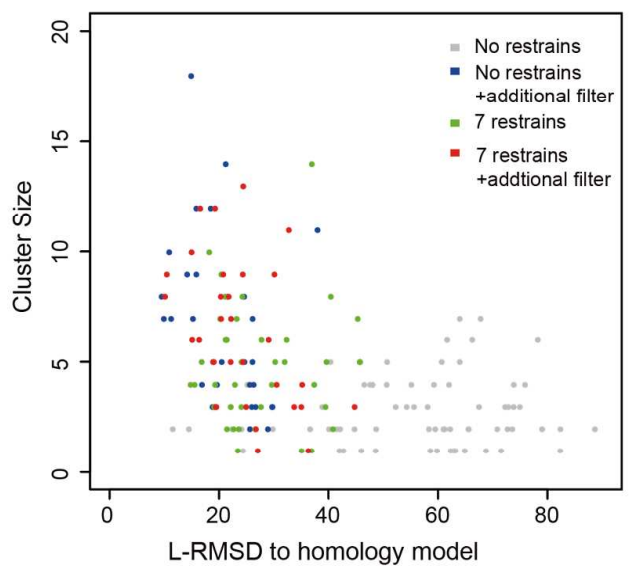

$b$

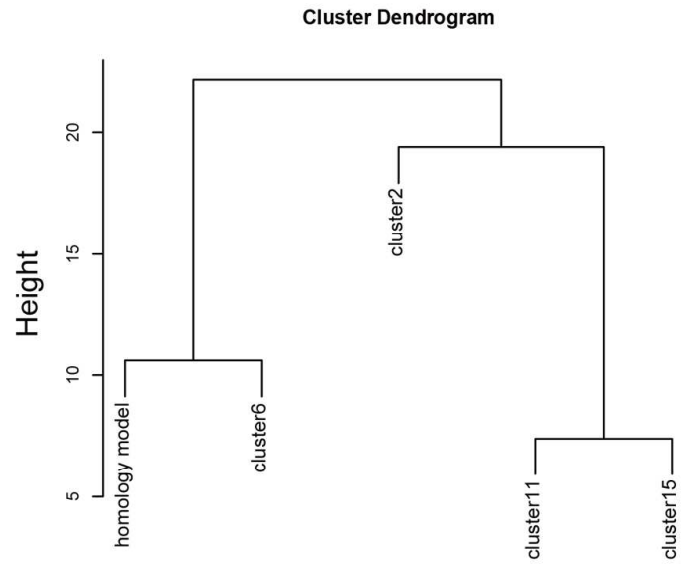




\section{Supplemental Figure 13. Ribosome cross-linking.}

(a) Comparison of the site pairs identified from the four amine-amine cross-linkers in the $70 \mathrm{~S}$ ribosome. (b-e) The $\mathrm{C}_{\alpha}-\mathrm{C}_{\alpha}$ distances of the cross-links were calculated based on the crystal structure 3OFA and 3OFC, and the distributions were plotted with a bin size of $5 \AA$. The green lines indicate a distance of $24 \AA$, and the red lines show the theoretical maximum $\mathrm{C}_{\alpha}-\mathrm{C}_{\alpha}$ distance of the corresponding cross-linker. 
Supplemental Figure 13

a

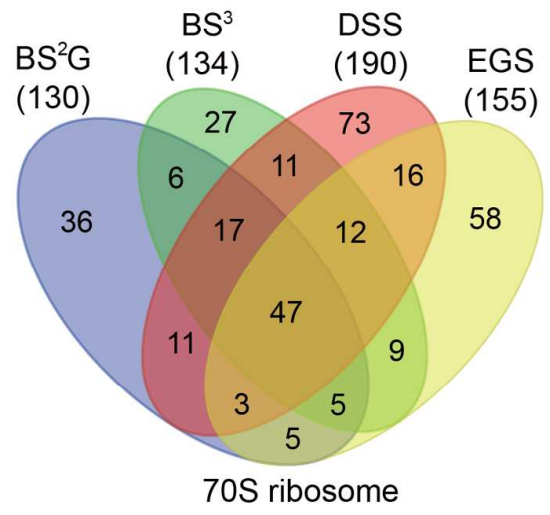

b

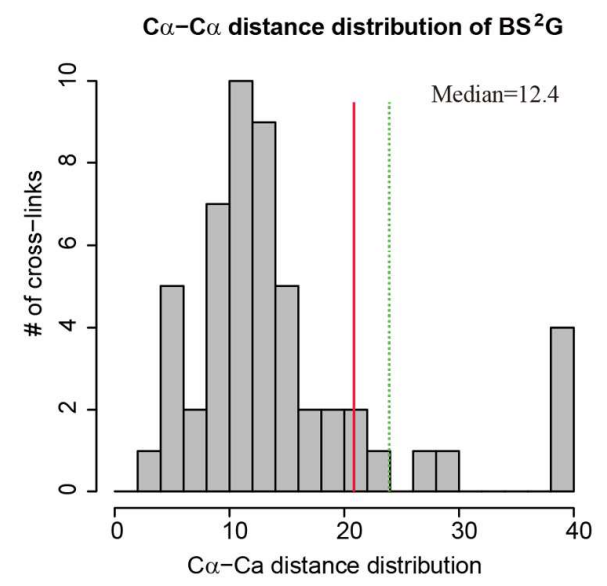

C

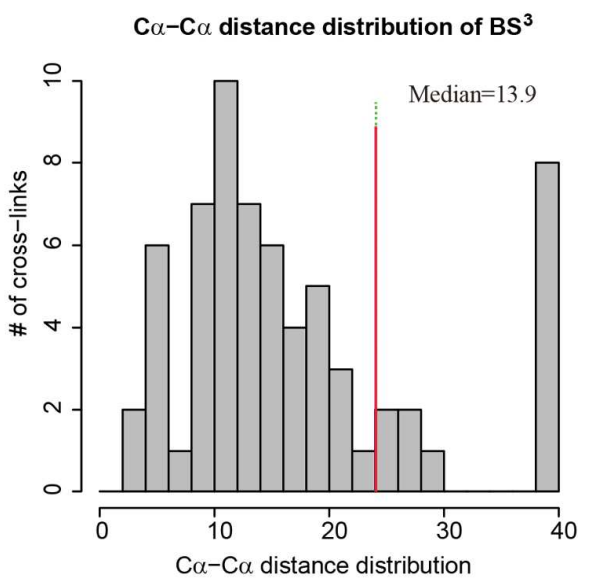

d $\mathrm{C} \alpha-\mathrm{C} \alpha$ distance distribution of DSS

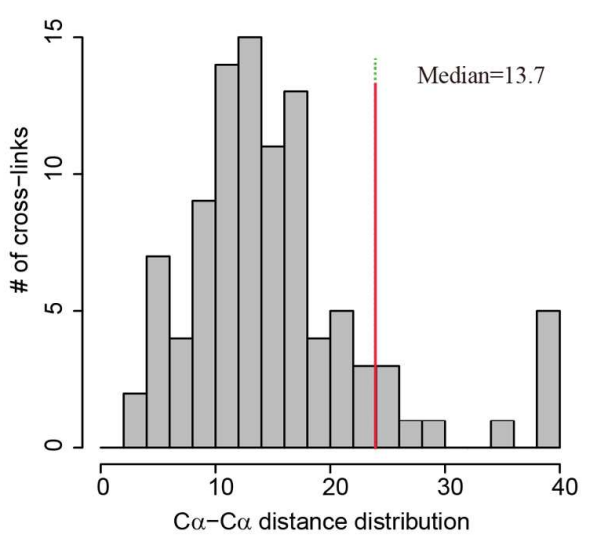

e

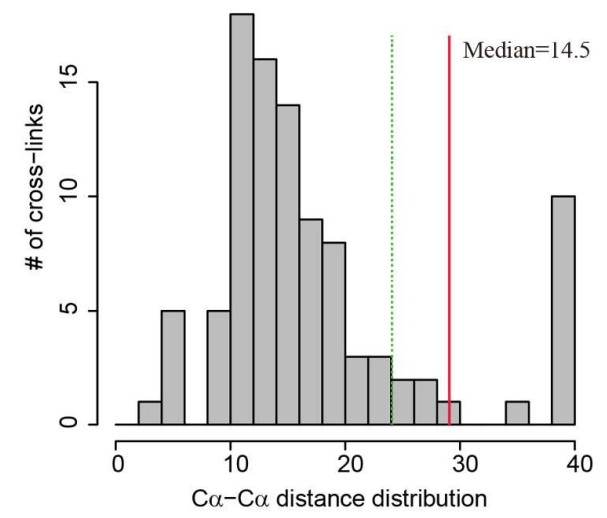




\section{Supplemental Figure 14. Cross-links between RL1, RL7, RL31, and RS1.}

The subunits cross-linked with RL1, RL7, RL31, or RS1 are colored, and the cross-linked residues are shown as spheres with the color of the cross-linker with which they were identified.

Supplemental Figure 14
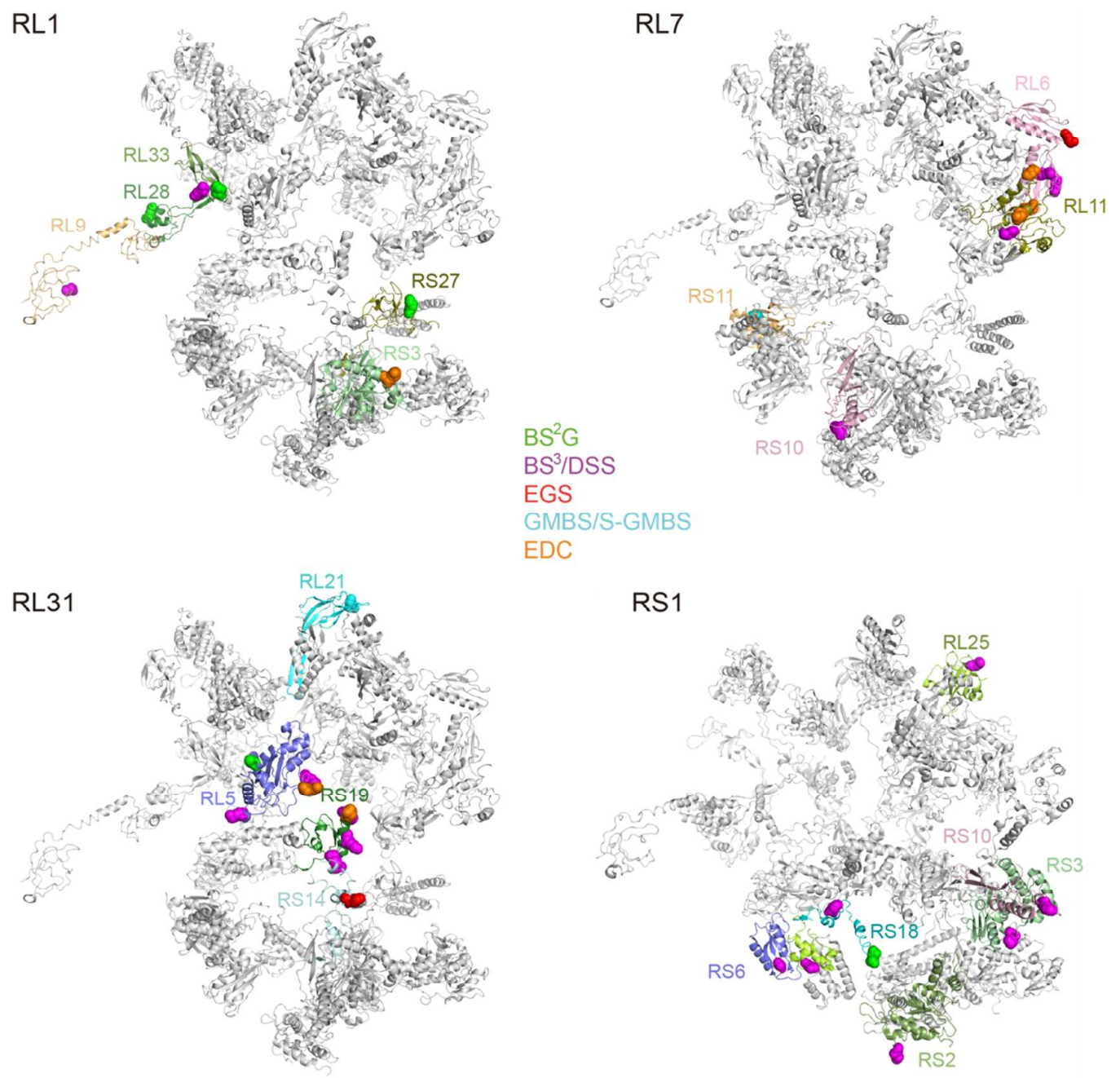


\section{Supplemental Figure 15. Two $\mathrm{BS}^{2} \mathrm{G}$-unique cross-links in Aldolase.}

Two BS ${ }^{2}$ G-unique cross-links (K138-K146 and K138-K107) represented using PyMOL. The cross-linked site pairs are close to each other, with a $\mathrm{C}_{\alpha}-\mathrm{C}_{\alpha}$ distance less than $14 \AA$.

Supplemental Figure 15

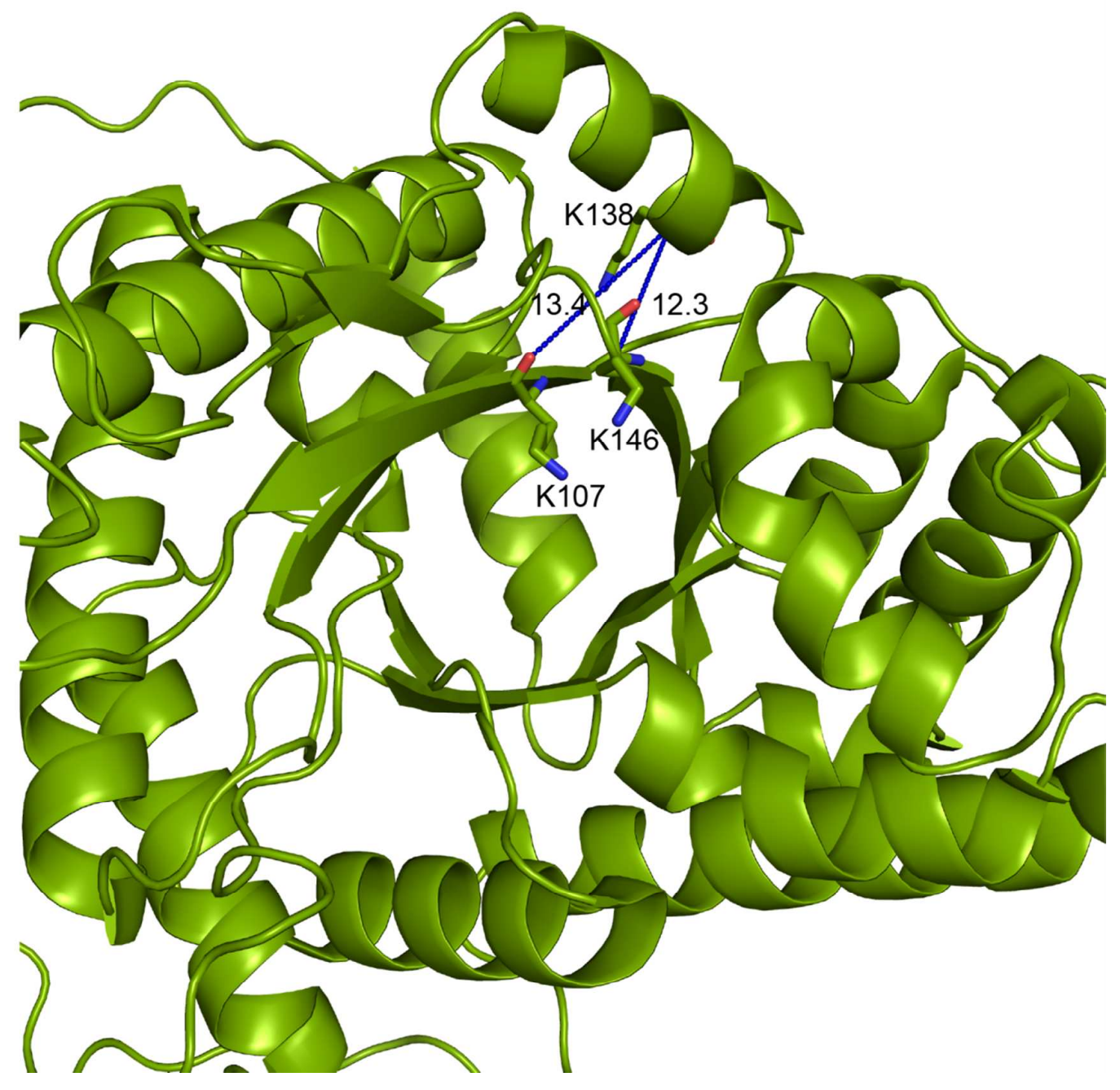




\section{Supplemental Figure 16. Representative $\mathrm{MS}^{2}$ spectra of the nine cross-linkers.}

$\mathrm{MS}^{2}$ spectra of cross-linked peptides for each cross-linker labeled with pLabel, with a $20 \mathrm{ppm}$ threshold for each peak. The cysteine colored in red indicates a Carbamidomethyl_C modification. Supplemental Figure 16
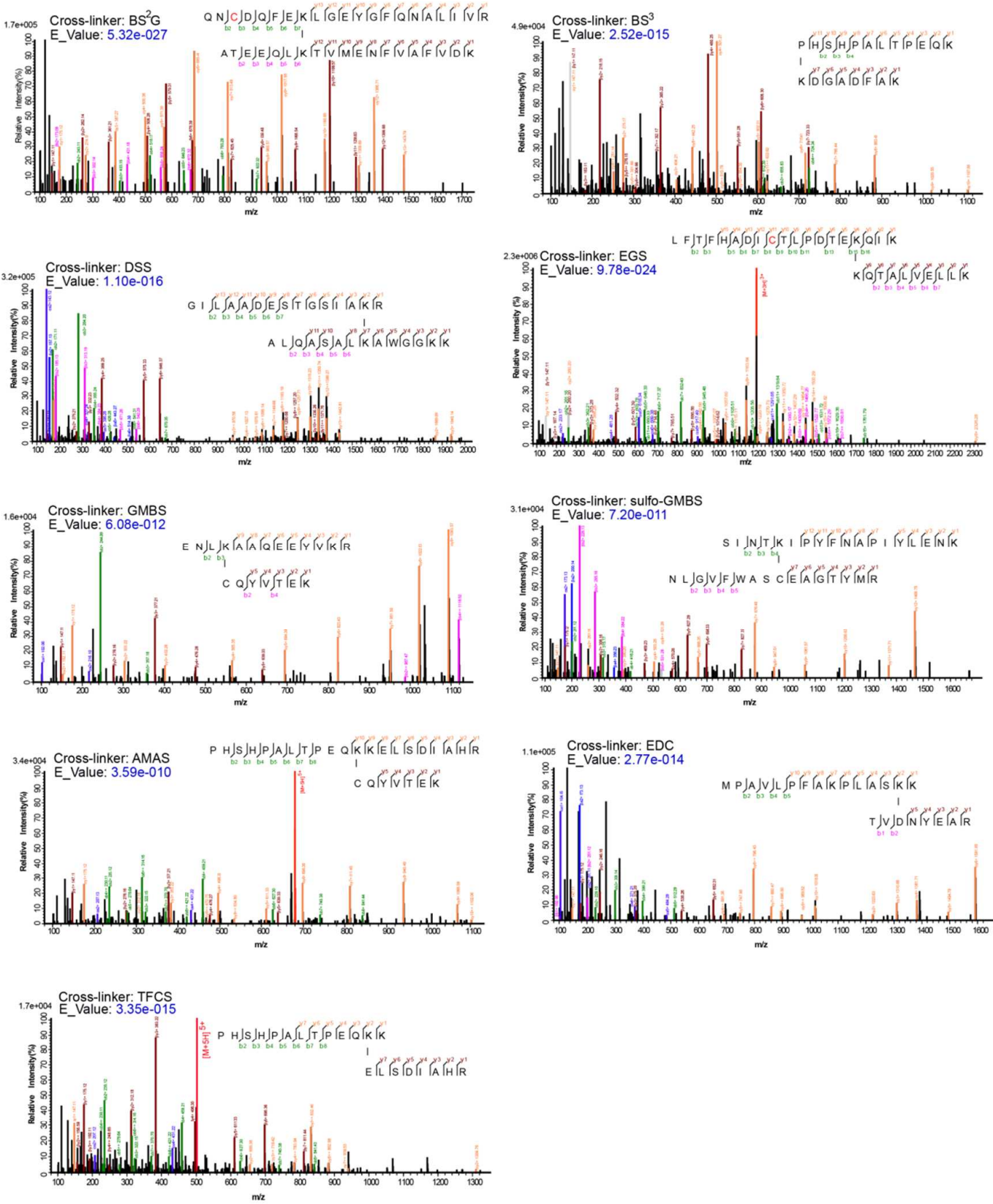


\section{Supplemental Table 1. Cross-links identified in BSA, Aldolase, and the CNGP complex.}

Adjacent residues were used to estimate the $\mathrm{C} \alpha$-C $\alpha$ distances when the cross-linked residues were missing in the PDB structure.

\begin{tabular}{|c|c|c|c|c|c|c|c|c|}
\hline \multicolumn{9}{|c|}{ AMAS } \\
\hline Site-pairs & Inter/Intra & $\begin{array}{c}\text { \#total } \\
\text { spec }\end{array}$ & \#Exp1 & \#Exp2 & \#Exp3 & \#Exp4 & Best E_value & Ca-Ca distance \\
\hline Aldolase(1)-Aldolase(201) & Inter & 74 & 14 & 18 & 22 & 20 & $3.2 \mathrm{E}-11$ & 10.2 \\
\hline Aldolase(98)-Aldolase(134) & Intra & 64 & 20 & 19 & 11 & 14 & $1.7 \mathrm{E}-09$ & 18.3 \\
\hline Aldolase(100)-Aldolase(134) & Intra & 51 & 14 & 19 & 9 & 9 & $3.6 \mathrm{E}-10$ & 23.1 \\
\hline Aldolase(134)-Aldolase(139) & Intra & 51 & 21 & 14 & 8 & 8 & $7.3 \mathrm{E}-09$ & 8.4 \\
\hline Aldolase(12)-Aldolase(201) & Inter & 45 & 11 & 13 & 10 & 11 & $1.3 \mathrm{E}-10$ & 19.3 \\
\hline Aldolase(41)-Aldolase(134) & Intra & 41 & 18 & 13 & 4 & 6 & $3.7 \mathrm{E}-14$ & 28.4 \\
\hline Aldolase(134)-Aldolase(146) & Intra & 36 & 15 & 11 & 5 & 5 & $3.8 \mathrm{E}-09$ & 11.2 \\
\hline Aldolase(134)-Aldolase(329) & Intra & 34 & 12 & 10 & 5 & 7 & $3.7 \mathrm{E}-11$ & 33.0 \\
\hline Aldolase(13)-Aldolase(134) & Intra & 21 & 6 & 7 & 2 & 6 & $1.6 \mathrm{E}-09$ & 18.2 \\
\hline Aldolase(134)-Aldolase(311) & Intra & 18 & 5 & 6 & 3 & 4 & $6.9 \mathrm{E}-06$ & 33.9 \\
\hline Aldolase(201)-Aldolase(316) & Intra & 17 & 4 & 4 & 4 & 5 & $4.7 \mathrm{E}-11$ & 40.7 \\
\hline Aldolase(110)-Aldolase(149) & Intra & 17 & 9 & 2 & 4 & 2 & $2.1 \mathrm{E}-04$ & 10.5 \\
\hline Aldolase(41)-Aldolase(149) & Intra & 16 & 4 & 4 & 4 & 4 & $1.1 \mathrm{E}-07$ & 21.1 \\
\hline Aldolase(41)-Aldolase(201) & Intra & 15 & 5 & 4 & 3 & 3 & $1.8 \mathrm{E}-13$ & 28.1 \\
\hline Aldolase(201)-Aldolase(329) & Intra & 15 & 4 & 4 & 3 & 4 & 7.4E-10 & 30.4 \\
\hline Aldolase(139)-Aldolase(149) & Intra & 15 & 4 & 4 & 3 & 4 & $3.5 \mathrm{E}-04$ & 25.9 \\
\hline Aldolase(149)-Aldolase(329) & Intra & 14 & 5 & 4 & 1 & 4 & $1.7 \mathrm{E}-11$ & 29.7 \\
\hline Aldolase(107)-Aldolase(149) & Intra & 14 & 4 & 5 & 2 & 3 & $9.2 \mathrm{E}-05$ & 12.2 \\
\hline Aldolase(134)-Aldolase(316) & Intra & 13 & 2 & 4 & 1 & 6 & $1.1 \mathrm{E}-07$ & 35.3 \\
\hline Aldolase(134)-Aldolase(317) & Intra & 12 & 3 & 3 & 2 & 4 & $1.8 \mathrm{E}-09$ & 34.3 \\
\hline Aldolase(27)-Aldolase(72) & Intra & 11 & 6 & - & 5 & - & $4.3 \mathrm{E}-16$ & 4.5 \\
\hline Aldolase(201)-Aldolase(311) & Intra & 11 & 4 & 6 & - & 1 & $5.1 \mathrm{E}-11$ & 31.5 \\
\hline Aldolase(146)-Aldolase(149) & Intra & 10 & 3 & 3 & 2 & 2 & $1.1 \mathrm{E}-04$ & 10.1 \\
\hline Aldolase(98)-Aldolase(149) & Intra & 10 & 4 & 5 & - & 1 & $1.9 \mathrm{E}-04$ & 27.7 \\
\hline Aldolase(100)-Aldolase(149) & Intra & 10 & 3 & 3 & 2 & 2 & $2.3 \mathrm{E}-04$ & 30.0 \\
\hline Aldolase(134)-Aldolase(321) & Intra & 8 & 3 & 5 & - & - & $1.2 \mathrm{E}-10$ & 34.7 \\
\hline Aldolase(201)-Aldolase(321) & Intra & 7 & 4 & - & 3 & - & $2.5 \mathrm{E}-09$ & 39.8 \\
\hline Aldolase(149)-Aldolase(316) & Intra & 7 & 3 & 1 & 3 & - & $2.0 \mathrm{E}-03$ & 34.6 \\
\hline Aldolase(139)-Aldolase(201) & Inter & 6 & - & 2 & 2 & 2 & $1.1 \mathrm{E}-12$ & 36.3 \\
\hline Aldolase(201)-Aldolase(317) & Intra & 5 & 3 & - & 2 & - & $4.0 \mathrm{E}-07$ & 42.2 \\
\hline Aldolase(149)-Aldolase(317) & Intra & 5 & 3 & 2 & - & - & $6.0 \mathrm{E}-04$ & 35.4 \\
\hline Aldolase(149)-Aldolase(311) & Intra & 4 & 2 & - & 2 & - & $2.9 \mathrm{E}-04$ & 28.5 \\
\hline bsa(368)-bsa(377) & Intra & 57 & 3 & 12 & 17 & 25 & $3.4 \mathrm{E}-11$ & 16.3 \\
\hline bsa(294)-bsa(315) & Intra & 51 & 12 & 21 & 12 & 6 & $2.6 \mathrm{E}-11$ & 32.5 \\
\hline
\end{tabular}




\begin{tabular}{|c|c|c|c|c|c|c|c|c|}
\hline bsa(471)-bsa(475) & Intra & 28 & 8 & 14 & 4 & 2 & $3.4 \mathrm{E}-12$ & 6.0 \\
\hline bsa(199)-bsa(465) & Intra & 22 & 2 & 11 & 5 & 4 & $1.5 \mathrm{E}-10$ & 15.5 \\
\hline bsa(359)-bsa(377) & Intra & 21 & 1 & 12 & 7 & 1 & $2.8 \mathrm{E}-11$ & 18.3 \\
\hline bsa(199)-bsa(211) & Intra & 19 & 2 & 3 & 6 & 8 & $1.8 \mathrm{E}-08$ & 12.1 \\
\hline bsa(159)-bsa(176) & Intra & 19 & 2 & 6 & 8 & 3 & $6.4 \mathrm{E}-06$ & 14.4 \\
\hline bsa(273)-bsa(277) & Intra & 18 & - & 12 & 5 & 1 & 4.2E-09 & 8.2 \\
\hline bsa(513)-bsa(524) & Intra & 15 & - & 3 & 6 & 6 & $4.0 \mathrm{E}-12$ & 12.0 \\
\hline bsa(93)-bsa(101) & Intra & 14 & 2 & 6 & 5 & 1 & $4.5 \mathrm{E}-10$ & 8.5 \\
\hline bsa(499)-bsa(513) & Intra & 12 & - & 3 & 4 & 5 & $1.3 \mathrm{E}-05$ & 28.2 \\
\hline bsa(315)-bsa(322) & Intra & 11 & 2 & 9 & - & - & $8.9 E-23$ & 9.3 \\
\hline bsa(471)-bsa(486) & Intra & 11 & 2 & 8 & 1 & - & $1.5 \mathrm{E}-06$ & 9.2 \\
\hline bsa(544)-bsa(557) & Intra & 10 & - & 5 & 2 & 3 & 8.9E-09 & 20.1 \\
\hline bsa(239)-bsa(244) & Intra & 10 & 1 & 2 & 2 & 5 & $3.1 \mathrm{E}-04$ & 8.5 \\
\hline bsa(280)-bsa(288) & Intra & 9 & 1 & 2 & 4 & 2 & $2.9 \mathrm{E}-07$ & 8.3 \\
\hline bsa(360)-bsa(377) & Intra & 8 & 7 & - & - & 1 & $1.3 \mathrm{E}-06$ & 21.1 \\
\hline bsa(359)-bsa(375) & Intra & 7 & - & 4 & 1 & 2 & $5.7 \mathrm{E}-08$ & 13.2 \\
\hline bsa(167)-bsa(180) & Intra & 6 & - & 4 & 2 & - & $9.2 \mathrm{E}-06$ & 9.5 \\
\hline bsa(273)-bsa(278) & Intra & 5 & - & - & 2 & 3 & $4.8 \mathrm{E}-07$ & 9.7 \\
\hline bsa(204)-bsa(460) & Intra & 4 & - & 1 & 3 & - & $3.2 \mathrm{E}-05$ & 10.9 \\
\hline bsa(90)-bsa(106) & Intra & 4 & 1 & 2 & - & 1 & $3.1 \mathrm{E}-04$ & 12.5 \\
\hline bsa(556)-bsa(566) & Intra & 4 & 1 & 2 & 1 & - & $3.8 \mathrm{E}-04$ & 8.3 \\
\hline Cbf5(114)-Cbf5(339) & Intra & 17 & 3 & 4 & 5 & 5 & $2.7 \mathrm{E}-07$ & 13.1 \\
\hline Gar1(77)-Gar1(94) & Intra & 17 & 5 & 4 & 5 & 3 & $6.1 \mathrm{E}-07$ & 6.8 \\
\hline Cbf5(137)-Cbf5(339) & Intra & 16 & 3 & 2 & 6 & 5 & $4.0 \mathrm{E}-07$ & 50.8 \\
\hline Cbf5(50)-Cbf5(339) & Intra & 13 & 1 & 1 & 7 & 4 & $1.5 \mathrm{E}-10$ & 21.0 \\
\hline Cbf5(180)-Cbf5(339) & Intra & 9 & 1 & - & 4 & 4 & $1.4 \mathrm{E}-05$ & 54.5 \\
\hline Cbf5(339)-Nop10(40) & Inter & 8 & - & - & 4 & 4 & $2.4 \mathrm{E}-10$ & 40.3 \\
\hline Cbf5(267)-Cbf5(339) & Intra & 7 & 2 & 1 & 3 & 1 & $1.8 \mathrm{E}-05$ & 16.2 \\
\hline Cbf5(339)-Cbf5(358) & Intra & 35 & 11 & 10 & 5 & 9 & $7.5 \mathrm{E}-17$ & 20.4[Cbf5(339)-Cbf5(350)] \\
\hline Cbf5(339)-Cbf5(383) & Intra & 19 & 5 & 5 & 5 & 4 & $1.2 \mathrm{E}-14$ & 20.4[Cbf5(339)-Cbf5(350)] \\
\hline Cbf5(339)-Cbf5(370) & Intra & 16 & 3 & 6 & 3 & 4 & $7.3 \mathrm{E}-10$ & 20.4[Cbf5(339)-Cbf5(350)] \\
\hline Cbf5(339)-Cbf5(367) & Intra & 6 & 3 & 3 & - & - & $5.4 \mathrm{E}-09$ & $\sim 20.4[\mathrm{Cbf5}(339)-\mathrm{Cbf5}(350)]$ \\
\hline \multicolumn{9}{|c|}{ GMBS } \\
\hline Site-pairs & Inter/Intra & $\begin{array}{c}\text { \#total } \\
\text { spec }\end{array}$ & \#Exp1 & \#Exp2 & \#Exp3 & \#Exp4 & Best E_value & Ca-Ca distance \\
\hline Aldolase(41)-Aldolase(134) & Intra & 137 & 35 & 36 & 35 & 31 & $2.6 \mathrm{E}-17$ & 28.4 \\
\hline Aldolase(100)-Aldolase(134) & Intra & 135 & 30 & 35 & 35 & 35 & $1.3 \mathrm{E}-12$ & 23.1 \\
\hline Aldolase(27)-Aldolase(134) & Intra & 99 & 25 & 25 & 28 & 21 & $5.5 \mathrm{E}-08$ & 24.4 \\
\hline Aldolase(98)-Aldolase(134) & Intra & 75 & 19 & 18 & 18 & 20 & $3.5 \mathrm{E}-11$ & 18.3 \\
\hline Aldolase(134)-Aldolase(329) & Intra & 71 & 19 & 17 & 20 & 15 & $8.6 \mathrm{E}-15$ & 33.0 \\
\hline Aldolase(134)-Aldolase(311) & Intra & 67 & 17 & 16 & 16 & 18 & 6.1E-09 & 33.9 \\
\hline
\end{tabular}




\begin{tabular}{|c|c|c|c|c|c|c|c|c|}
\hline Aldolase(134)-Aldolase(139) & Intra & 66 & 18 & 14 & 18 & 16 & $4.2 \mathrm{E}-11$ & 8.4 \\
\hline Aldolase(134)-Aldolase(146) & Intra & 55 & 13 & 18 & 11 & 13 & $3.0 \mathrm{E}-11$ & 11.2 \\
\hline Aldolase(27)-Aldolase(201) & Intra & 49 & 14 & 14 & 11 & 10 & $4.3 \mathrm{E}-11$ & 26.9 \\
\hline Aldolase(12)-Aldolase(201) & Inter & 47 & 15 & 15 & 9 & 8 & $7.4 \mathrm{E}-12$ & 19.3 \\
\hline Aldolase(1)-Aldolase(201) & Inter & 44 & 12 & 10 & 13 & 9 & $1.7 \mathrm{E}-10$ & 10.2 \\
\hline Aldolase(134)-Aldolase(316) & Intra & 44 & 11 & 11 & 12 & 10 & $5.0 \mathrm{E}-08$ & 35.3 \\
\hline Aldolase(201)-Aldolase(311) & Intra & 39 & 9 & 10 & 9 & 11 & $2.1 \mathrm{E}-12$ & 31.5 \\
\hline Aldolase(100)-Aldolase(149) & Intra & 38 & 12 & 8 & 12 & 6 & $4.9 \mathrm{E}-07$ & 30.0 \\
\hline Aldolase(41)-Aldolase(201) & Intra & 33 & 7 & 11 & 7 & 8 & $4.3 \mathrm{E}-15$ & 28.1 \\
\hline Aldolase(149)-Aldolase(329) & Intra & 33 & 8 & 10 & 10 & 5 & $8.5 \mathrm{E}-10$ & 29.7 \\
\hline Aldolase(146)-Aldolase(149) & Intra & 33 & 12 & 5 & 10 & 6 & $1.7 \mathrm{E}-06$ & 10.1 \\
\hline Aldolase(201)-Aldolase(329) & Intra & 31 & 6 & 7 & 9 & 9 & $1.3 \mathrm{E}-14$ & 30.4 \\
\hline Aldolase(201)-Aldolase(321) & Intra & 30 & 7 & 8 & 7 & 8 & $2.3 \mathrm{E}-12$ & 39.8 \\
\hline Aldolase(134)-Aldolase(321) & Intra & 30 & 6 & 9 & 9 & 6 & 4.1E-06 & 34.7 \\
\hline Aldolase(110)-Aldolase(149) & Intra & 28 & 8 & 7 & 7 & 6 & $6.1 \mathrm{E}-04$ & 10.5 \\
\hline Aldolase(329)-Aldolase(338) & Intra & 27 & 7 & 7 & 7 & 6 & $1.1 \mathrm{E}-11$ & 14.4 \\
\hline Aldolase(13)-Aldolase(134) & Intra & 27 & 8 & 7 & 5 & 7 & $3.2 \mathrm{E}-10$ & 18.2 \\
\hline Aldolase(134)-Aldolase(317) & Intra & 25 & 7 & 5 & 7 & 6 & 2.7E-10 & 34.3 \\
\hline Aldolase(201)-Aldolase(316) & Intra & 22 & 6 & 5 & 6 & 5 & $3.3 \mathrm{E}-12$ & 40.7 \\
\hline Aldolase(41)-Aldolase(149) & Intra & 22 & 9 & 4 & 5 & 4 & $1.2 \mathrm{E}-07$ & 21.1 \\
\hline Aldolase(98)-Aldolase(201) & Intra & 20 & 6 & 6 & 4 & 4 & 7.0E-12 & 37.0 \\
\hline Aldolase(139)-Aldolase(149) & Intra & 19 & 5 & 4 & 5 & 5 & $1.5 \mathrm{E}-06$ & 25.9 \\
\hline Aldolase(110)-Aldolase(134) & Intra & 18 & 8 & 4 & 4 & 2 & $2.2 \mathrm{E}-07$ & 13.7 \\
\hline Aldolase(27)-Aldolase(72) & Intra & 16 & 6 & 9 & 1 & - & $1.9 \mathrm{E}-11$ & 4.5 \\
\hline Aldolase(107)-Aldolase(149) & Intra & 15 & 3 & 5 & 3 & 4 & $5.2 \mathrm{E}-05$ & 12.2 \\
\hline Aldolase(139)-Aldolase(201) & Inter & 14 & 5 & 3 & 3 & 3 & $1.1 \mathrm{E}-15$ & 36.3 \\
\hline Aldolase(100)-Aldolase(201) & Intra & 14 & 4 & 3 & 3 & 4 & $3.3 \mathrm{E}-13$ & 37.2 \\
\hline Aldolase(149)-Aldolase(311) & Intra & 14 & 5 & 5 & 2 & 2 & $5.8 \mathrm{E}-05$ & 28.5 \\
\hline Aldolase(201)-Aldolase(317) & Intra & 13 & 3 & 3 & 4 & 3 & $1.5 \mathrm{E}-07$ & 42.2 \\
\hline Aldolase(149)-Aldolase(317) & Intra & 12 & 2 & 4 & 3 & 3 & $8.4 \mathrm{E}-06$ & 35.4 \\
\hline Aldolase(98)-Aldolase(149) & Intra & 11 & 5 & 2 & 2 & 2 & $2.3 \mathrm{E}-04$ & 27.7 \\
\hline Aldolase(110)-Aldolase(201) & Intra & 9 & 2 & 2 & 1 & 4 & $1.7 \mathrm{E}-05$ & 26.6 \\
\hline Aldolase(149)-Aldolase(316) & Intra & 9 & 3 & 1 & 3 & 2 & $9.5 \mathrm{E}-05$ & 34.6 \\
\hline Aldolase(41)-Aldolase(338) & Intra & 8 & 1 & 3 & 1 & 3 & $1.9 \mathrm{E}-08$ & 29.1 \\
\hline Aldolase(107)-Aldolase(134) & Intra & 7 & 1 & 1 & 3 & 2 & $9.8 \mathrm{E}-08$ & 10.2 \\
\hline Aldolase(12)-Aldolase(134) & Intra & 7 & 4 & 2 & 1 & - & $2.2 \mathrm{E}-07$ & 16.6 \\
\hline Aldolase(27)-Aldolase(338) & Intra & 7 & 4 & 2 & 1 & - & $3.5 \mathrm{E}-06$ & 7.3 \\
\hline Aldolase(139)-Aldolase(338) & Intra & 5 & 2 & 3 & - & - & $1.6 \mathrm{E}-10$ & 30.7 \\
\hline Aldolase(12)-Aldolase(149) & Intra & 5 & 2 & 2 & 1 & - & 6.7E-04 & 25.1 \\
\hline Aldolase(1)-Aldolase(149) & Inter & 5 & 1 & 2 & 1 & 1 & 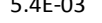 & 17.3 \\
\hline
\end{tabular}




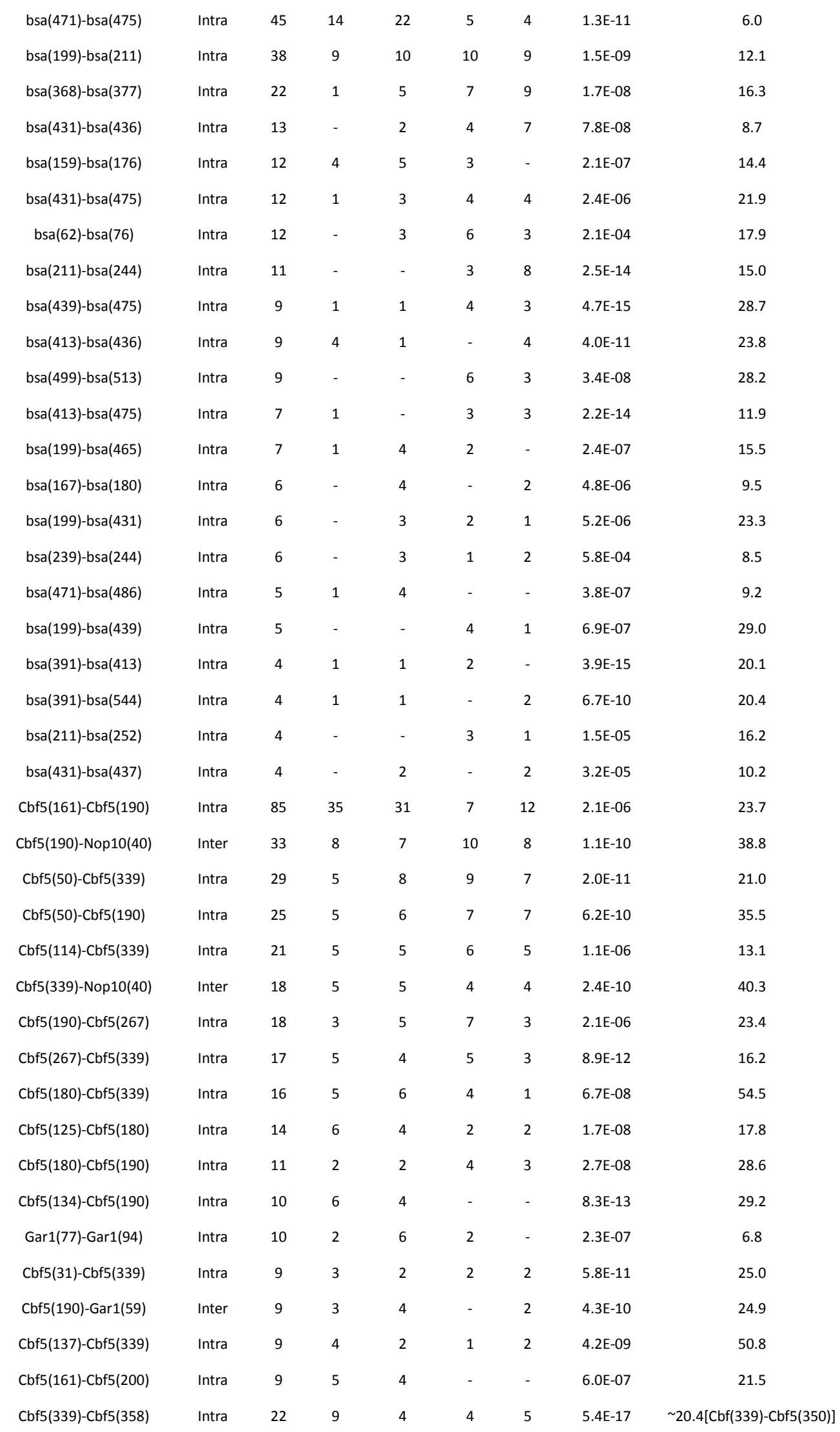




\begin{tabular}{|c|c|c|c|c|c|c|c|c|}
\hline Cbf5(339)-Cbf5(370) & Intra & 13 & 3 & 3 & 4 & 3 & $2.3 \mathrm{E}-12$ & $\sim 20.4[\mathrm{Cbf}(339)-\mathrm{Cbf5}(350)]$ \\
\hline Cbf5(339)-Cbf5(383) & Intra & 8 & 3 & - & 3 & 2 & 2.0E-08 & $\sim 20.4[\mathrm{Cbf}(339)-\mathrm{Cbf5}(350)]$ \\
\hline \multicolumn{9}{|c|}{ Sulfo-GMBS } \\
\hline Site-pairs & Inter/Intra & $\begin{array}{c}\text { \#total } \\
\text { spec }\end{array}$ & \#Exp1 & \#Exp2 & \#Exp3 & \#Exp4 & Best E_value & Ca-Ca distance \\
\hline Aldolase(100)-Aldolase(134) & Intra & 129 & 26 & 33 & 36 & 34 & $5.5 \mathrm{E}-11$ & 23.1 \\
\hline Aldolase(41)-Aldolase(134) & Intra & 118 & 26 & 31 & 29 & 32 & $4.2 \mathrm{E}-22$ & 28.4 \\
\hline Aldolase(27)-Aldolase(134) & Intra & 99 & 20 & 21 & 29 & 29 & $1.6 \mathrm{E}-08$ & 24.4 \\
\hline Aldolase(1)-Aldolase(201) & Inter & 94 & 20 & 23 & 25 & 26 & $3.3 \mathrm{E}-12$ & 10.2 \\
\hline Aldolase(134)-Aldolase(329) & Intra & 83 & 19 & 16 & 22 & 26 & $1.0 \mathrm{E}-19$ & 33.0 \\
\hline Aldolase(98)-Aldolase(134) & Intra & 77 & 20 & 19 & 19 & 19 & $5.6 \mathrm{E}-10$ & 18.3 \\
\hline Aldolase(134)-Aldolase(311) & Intra & 77 & 14 & 20 & 22 & 21 & 4.6E-09 & 33.9 \\
\hline Aldolase(134)-Aldolase(139) & Intra & 72 & 22 & 16 & 18 & 16 & $1.5 \mathrm{E}-11$ & 8.4 \\
\hline Aldolase(134)-Aldolase(316) & Intra & 63 & 11 & 13 & 19 & 20 & $3.2 \mathrm{E}-08$ & 35.3 \\
\hline Aldolase(134)-Aldolase(146) & Intra & 54 & 12 & 14 & 12 & 16 & $5.9 \mathrm{E}-11$ & 11.2 \\
\hline Aldolase(201)-Aldolase(321) & Intra & 49 & 11 & 12 & 14 & 12 & $8.1 \mathrm{E}-12$ & 39.8 \\
\hline Aldolase(12)-Aldolase(201) & Inter & 47 & 10 & 6 & 17 & 14 & $1.2 \mathrm{E}-11$ & 19.3 \\
\hline Aldolase(27)-Aldolase(201) & Intra & 46 & 11 & 8 & 14 & 13 & $3.4 \mathrm{E}-11$ & 26.9 \\
\hline Aldolase(13)-Aldolase(134) & Intra & 46 & 9 & 13 & 12 & 12 & 4.6E-10 & 18.2 \\
\hline Aldolase(107)-Aldolase(134) & Intra & 45 & 6 & 9 & 14 & 16 & $2.3 \mathrm{E}-12$ & 10.2 \\
\hline Aldolase(201)-Aldolase(311) & Intra & 42 & 10 & 11 & 11 & 10 & $5.7 \mathrm{E}-13$ & 31.5 \\
\hline Aldolase(134)-Aldolase(321) & Intra & 42 & 6 & 11 & 13 & 12 & $1.8 \mathrm{E}-06$ & 34.7 \\
\hline Aldolase(100)-Aldolase(149) & Intra & 41 & 10 & 9 & 10 & 12 & $3.2 \mathrm{E}-07$ & 30.0 \\
\hline Aldolase(41)-Aldolase(201) & Intra & 38 & 7 & 8 & 10 & 13 & $2.6 \mathrm{E}-15$ & 28.1 \\
\hline Aldolase(149)-Aldolase(329) & Intra & 37 & 6 & 8 & 12 & 11 & $1.0 \mathrm{E}-09$ & 29.7 \\
\hline Aldolase(201)-Aldolase(329) & Intra & 36 & 10 & 8 & 9 & 9 & $4.9 \mathrm{E}-14$ & 30.4 \\
\hline Aldolase(201)-Aldolase(316) & Intra & 35 & 9 & 6 & 10 & 10 & $2.3 \mathrm{E}-13$ & 40.7 \\
\hline Aldolase(134)-Aldolase(317) & Intra & 33 & 8 & 8 & 10 & 7 & $4.9 \mathrm{E}-10$ & 34.3 \\
\hline Aldolase(329)-Aldolase(338) & Intra & 33 & 8 & 9 & 7 & 9 & $3.3 \mathrm{E}-09$ & 14.4 \\
\hline Aldolase(107)-Aldolase(149) & Intra & 32 & 9 & 6 & 10 & 7 & $3.0 \mathrm{E}-05$ & 12.2 \\
\hline Aldolase(27)-Aldolase(72) & Intra & 31 & 1 & - & 20 & 10 & $7.2 \mathrm{E}-16$ & 4.5 \\
\hline Aldolase(41)-Aldolase(72) & Intra & 30 & - & - & 16 & 14 & $2.0 \mathrm{E}-05$ & 27.0 \\
\hline Aldolase(149)-Aldolase(311) & Intra & 30 & 6 & 6 & 10 & 8 & 5.7E-04 & 28.5 \\
\hline Aldolase(1)-Aldolase(134) & Intra & 28 & - & 5 & 11 & 12 & $1.6 \mathrm{E}-08$ & 29.0 \\
\hline Aldolase(98)-Aldolase(201) & Intra & 27 & 6 & 6 & 6 & 9 & $7.6 \mathrm{E}-13$ & 37.0 \\
\hline Aldolase(41)-Aldolase(338) & Intra & 24 & 3 & 7 & 7 & 7 & $4.2 \mathrm{E}-13$ & 29.1 \\
\hline Aldolase(100)-Aldolase(201) & Intra & 24 & 4 & 3 & 9 & 8 & $3.6 \mathrm{E}-12$ & 37.2 \\
\hline Aldolase(41)-Aldolase(149) & Intra & 22 & 4 & 4 & 8 & 6 & $1.2 \mathrm{E}-07$ & 21.1 \\
\hline Aldolase(146)-Aldolase(149) & Intra & 22 & 8 & 5 & 5 & 4 & $1.0 \mathrm{E}-06$ & 10.1 \\
\hline Aldolase(201)-Aldolase(317) & Intra & 21 & 4 & 4 & 6 & 7 & $1.8 \mathrm{E}-09$ & 42.2 \\
\hline Aldolase(110)-Aldolase(134) & Intra & 19 & 6 & 1 & 6 & 6 & $2.6 \mathrm{E}-08$ & 13.7 \\
\hline
\end{tabular}




\begin{tabular}{|c|c|c|c|c|c|c|c|c|}
\hline Aldolase(149)-Aldolase(316) & Intra & 19 & 4 & 4 & 6 & 5 & $4.8 \mathrm{E}-05$ & 34.6 \\
\hline Aldolase(98)-Aldolase(149) & Intra & 18 & 3 & 4 & 6 & 5 & $2.0 \mathrm{E}-05$ & 27.7 \\
\hline Aldolase(139)-Aldolase(149) & Intra & 17 & 3 & 5 & 4 & 5 & $6.2 \mathrm{E}-06$ & 25.9 \\
\hline Aldolase(149)-Aldolase(317) & Intra & 16 & 4 & 3 & 3 & 6 & $5.4 \mathrm{E}-05$ & 35.4 \\
\hline Aldolase(139)-Aldolase(201) & Inter & 13 & 3 & 3 & 4 & 3 & $6.7 \mathrm{E}-15$ & 36.3 \\
\hline Aldolase(27)-Aldolase(338) & Intra & 12 & 1 & 1 & 8 & 2 & $7.7 \mathrm{E}-08$ & 7.3 \\
\hline Aldolase(12)-Aldolase(134) & Intra & 12 & 1 & 3 & 3 & 5 & $1.3 \mathrm{E}-07$ & 16.6 \\
\hline Aldolase(110)-Aldolase(149) & Intra & 10 & 3 & 4 & 1 & 2 & $7.3 \mathrm{E}-04$ & 10.5 \\
\hline Aldolase(139)-Aldolase(338) & Intra & 8 & 2 & - & 3 & 3 & $7.3 \mathrm{E}-11$ & 30.7 \\
\hline Aldolase(321)-Aldolase(338) & Intra & 8 & - & 2 & 3 & 3 & $2.3 \mathrm{E}-07$ & 25.6 \\
\hline Aldolase(13)-Aldolase(149) & Intra & 8 & 3 & - & 1 & 4 & $2.8 \mathrm{E}-03$ & 27.2 \\
\hline Aldolase(149)-Aldolase(321) & Intra & 8 & 2 & 2 & 1 & 3 & $4.5 \mathrm{E}-03$ & 35.3 \\
\hline Aldolase(146)-Aldolase(201) & Intra & 7 & 2 & - & 3 & 2 & $3.3 \mathrm{E}-10$ & 23.2 \\
\hline Aldolase(13)-Aldolase(201) & Inter & 6 & 3 & - & 3 & - & $1.8 \mathrm{E}-11$ & 21.6 \\
\hline Aldolase(110)-Aldolase(338) & Intra & 6 & 1 & 1 & 2 & 2 & $2.4 \mathrm{E}-06$ & 29.8 \\
\hline Aldolase(98)-Aldolase(239) & Intra & 6 & 2 & 2 & 2 & - & $2.3 \mathrm{E}-05$ & 42.0 \\
\hline Aldolase(1)-Aldolase(149) & Inter & 6 & - & - & 4 & 2 & $4.8 \mathrm{E}-04$ & 17.3 \\
\hline Aldolase(110)-Aldolase(201) & Intra & 5 & - & 1 & 1 & 3 & $8.2 \mathrm{E}-05$ & 26.6 \\
\hline Aldolase(311)-Aldolase(338) & Intra & 4 & - & 1 & 3 & - & $2.9 \mathrm{E}-06$ & 24.8 \\
\hline Aldolase(12)-Aldolase(149) & Intra & 4 & - & - & 3 & 1 & $5.1 \mathrm{E}-03$ & 25.1 \\
\hline bsa(471)-bsa(475) & Intra & 31 & 15 & 13 & 3 & - & $1.9 \mathrm{E}-14$ & 6.0 \\
\hline bsa(199)-bsa(211) & Intra & 31 & 7 & 12 & 7 & 5 & $2.2 \mathrm{E}-09$ & 12.1 \\
\hline bsa(368)-bsa(377) & Intra & 28 & 1 & 3 & 11 & 13 & $9.0 \mathrm{E}-07$ & 16.3 \\
\hline bsa(159)-bsa(176) & Intra & 22 & 4 & 11 & 4 & 3 & $3.0 \mathrm{E}-07$ & 14.4 \\
\hline bsa(431)-bsa(436) & Intra & 21 & 4 & 2 & 9 & 6 & $2.4 \mathrm{E}-08$ & 8.7 \\
\hline bsa(211)-bsa(244) & Intra & 15 & 3 & 1 & 6 & 5 & $7.3 \mathrm{E}-15$ & 15.0 \\
\hline bsa(62)-bsa(76) & Intra & 15 & 1 & 4 & 7 & 3 & $4.9 \mathrm{E}-05$ & 17.9 \\
\hline bsa(431)-bsa(475) & Intra & 14 & 2 & 3 & 4 & 5 & $3.9 \mathrm{E}-06$ & 21.9 \\
\hline bsa(471)-bsa(486) & Intra & 13 & 2 & 7 & 3 & 1 & $1.9 \mathrm{E}-06$ & 9.2 \\
\hline bsa(413)-bsa(475) & Intra & 11 & 1 & 2 & 4 & 4 & $6.2 \mathrm{E}-15$ & 11.9 \\
\hline bsa(439)-bsa(475) & Intra & 10 & 2 & - & 4 & 4 & $4.3 \mathrm{E}-14$ & 28.7 \\
\hline bsa(199)-bsa(465) & Intra & 10 & 6 & 4 & - & - & $2.0 \mathrm{E}-09$ & 15.5 \\
\hline bsa(413)-bsa(436) & Intra & 8 & 2 & 4 & - & 2 & $1.2 \mathrm{E}-17$ & 23.8 \\
\hline bsa(377)-bsa(475) & Intra & 7 & - & - & 1 & 6 & $5.6 \mathrm{E}-15$ & 23.4 \\
\hline bsa(350)-bsa(475) & Intra & 7 & - & - & 5 & 2 & $2.7 \mathrm{E}-14$ & 16.2 \\
\hline bsa(465)-bsa(475) & Intra & 7 & - & - & 2 & 5 & $1.2 \mathrm{E}-09$ & 15.2 \\
\hline bsa(167)-bsa(180) & Intra & 7 & 1 & 3 & 1 & 2 & $6.4 \mathrm{E}-09$ & 9.5 \\
\hline bsa(199)-bsa(431) & Intra & 7 & - & 3 & 2 & 2 & $1.7 \mathrm{E}-06$ & 23.3 \\
\hline bsa(294)-bsa(436) & Intra & 7 & 2 & 2 & 2 & 1 & $4.7 \mathrm{E}-06$ & 18.9 \\
\hline bsa(239)-bsa(244) & Intra & 7 & - & 2 & 4 & 1 & $1.6 \mathrm{E}-04$ & 8.5 \\
\hline
\end{tabular}




\begin{tabular}{|c|c|c|c|c|c|c|c|c|}
\hline \\
\hline bsa(273)-bsa(277) & Intra & 6 & 1 & 5 & - & - & $4.3 \mathrm{E}-07$ & 8.2 \\
\hline bsa(396)-bsa(475) & Intra & 6 & - & - & 2 & 4 & $1.9 \mathrm{E}-05$ & 29.2 \\
\hline bsa(211)-bsa(252) & Intra & 6 & - & - & 2 & 4 & $3.4 \mathrm{E}-05$ & 16.2 \\
\hline bsa(499)-bsa(513) & Intra & 6 & - & - & 5 & 1 & $6.5 \mathrm{E}-04$ & 28.2 \\
\hline bsa(12)-bsa(475) & Intra & 5 & - & - & 3 & 2 & $1.8 \mathrm{E}-14$ & 45.2 \\
\hline bsa(76)-bsa(475) & Intra & 5 & - & - & 3 & 2 & $6.5 \mathrm{E}-12$ & 40.6 \\
\hline bsa(294)-bsa(315) & Intra & 5 & 1 & 3 & 1 & - & $1.2 \mathrm{E}-10$ & 32.5 \\
\hline bsa(436)-bsa(439) & Intra & 5 & - & - & 4 & 1 & $1.1 \mathrm{E}-08$ & 5.4 \\
\hline bsa(199)-bsa(439) & Intra & 5 & - & 1 & 2 & 2 & $2.2 \mathrm{E}-06$ & 29.0 \\
\hline bsa(431)-bsa(437) & Intra & 5 & - & 4 & - & 1 & 8.7E-05 & 10.2 \\
\hline bsa(391)-bsa(413) & Intra & 4 & 2 & 1 & - & 1 & $1.1 \mathrm{E}-21$ & 20.1 \\
\hline bsa(391)-bsa(544) & Intra & 4 & 3 & 1 & - & - & $5.6 \mathrm{E}-11$ & 20.4 \\
\hline Cbf5(161)-Cbf5(190) & Intra & 53 & 10 & 13 & 14 & 16 & $1.2 \mathrm{E}-05$ & 23.7 \\
\hline Gar1(77)-Gar1(94) & Intra & 29 & 8 & 5 & 9 & 7 & $1.9 \mathrm{E}-09$ & 6.8 \\
\hline Cbf5(161)-Cbf5(200) & Intra & 29 & 22 & 7 & - & - & $4.9 \mathrm{E}-08$ & 21.5 \\
\hline Cbf5(114)-Cbf5(339) & Intra & 28 & 5 & 6 & 9 & 8 & $3.2 \mathrm{E}-08$ & 13.1 \\
\hline Cbf5(50)-Cbf5(339) & Intra & 26 & 7 & 6 & 8 & 5 & $6.0 \mathrm{E}-12$ & 21.0 \\
\hline Cbf5(190)-Gar1(59) & Inter & 19 & 7 & 4 & 5 & 3 & $4.3 \mathrm{E}-11$ & 24.9 \\
\hline Cbf5(339)-Nop10(40) & Inter & 19 & 5 & 5 & 4 & 5 & $6.1 \mathrm{E}-11$ & 40.3 \\
\hline Cbf5(125)-Nop10(40) & Inter & 19 & 4 & 3 & 5 & 7 & $1.3 \mathrm{E}-10$ & 32.6 \\
\hline Cbf5(125)-Cbf5(180) & Intra & 19 & 7 & 3 & 2 & 7 & $2.1 \mathrm{E}-09$ & 17.8 \\
\hline Cbf5(190)-Nop10(40) & Inter & 18 & 8 & 4 & 2 & 4 & $3.5 \mathrm{E}-10$ & 38.8 \\
\hline Cbf5(50)-Cbf5(190) & Intra & 16 & 5 & 3 & 4 & 4 & $2.8 \mathrm{E}-08$ & 35.5 \\
\hline Cbf5(180)-Cbf5(339) & Intra & 15 & 4 & 4 & 4 & 3 & $2.2 \mathrm{E}-08$ & 54.5 \\
\hline Gar1(94)-Nop10(40) & Inter & 13 & 2 & 3 & 4 & 4 & $9.0 \mathrm{E}-07$ & 56.2 \\
\hline Cbf5(267)-Cbf5(339) & Intra & 12 & 2 & 3 & 4 & 3 & $9.7 \mathrm{E}-08$ & 16.2 \\
\hline Cbf5(134)-Cbf5(339) & Intra & 11 & - & 1 & 3 & 7 & $1.0 \mathrm{E}-06$ & 57.1 \\
\hline Cbf5(125)-Cbf5(134) & Intra & 10 & 6 & 4 & - & - & $1.9 \mathrm{E}-06$ & 22.1 \\
\hline Cbf5(137)-Cbf5(339) & Intra & 9 & 3 & 3 & 3 & - & $2.1 \mathrm{E}-08$ & 50.8 \\
\hline Cbf5(134)-Cbf5(190) & Intra & 8 & 5 & 3 & - & - & $1.4 \mathrm{E}-13$ & 29.2 \\
\hline Cbf5(31)-Cbf5(339) & Intra & 6 & 3 & 1 & 1 & 1 & $9.0 \mathrm{E}-11$ & 25.0 \\
\hline Cbf5(50)-Gar1(94) & Inter & 6 & - & - & 3 & 3 & 2.7E-08 & 65.9 \\
\hline Cbf5(339)-Nop10(19) & Inter & 6 & 2 & - & 3 & 1 & 8.7E-06 & 48.6 \\
\hline Cbf5(114)-Cbf5(190) & Intra & 5 & - & - & 1 & 4 & $3.9 \mathrm{E}-08$ & 17.1 \\
\hline Cbf5(161)-Gar1(94) & Inter & 5 & - & - & 2 & 3 & 1.7E-04 & 23.3 \\
\hline Cbf5(190)-Cbf5(267) & Intra & 4 & 1 & 1 & - & 2 & $4.2 \mathrm{E}-06$ & 23.4 \\
\hline Cbf5(50)-Cbf5(125) & Intra & 4 & - & - & 3 & 1 & $7.5 \mathrm{E}-04$ & 39.0 \\
\hline & & & & $\mathrm{BS}^{2} \mathrm{G}$ & & & & \\
\hline Site-pairs & Inter/Intra & $\begin{array}{c}\text { \#total } \\
\text { spec }\end{array}$ & \#Exp1 & \#Exp2 & \#Еxp3 & \#Exp4 & Best E_value & Ca-Ca distance \\
\hline dolase(41)-Aldolase(311) & Intra & 189 & 32 & 70 & 45 & 42 & $1.53 \mathrm{E}-16$ & 12.7 \\
\hline
\end{tabular}




\begin{tabular}{|c|c|c|c|c|c|c|c|c|}
\hline Aldolase(98)-Aldolase(138) & Intra & 71 & 13 & 21 & 20 & 17 & $9.31 \mathrm{E}-17$ & 13.4 \\
\hline Aldolase(311)-Aldolase(317) & Intra & 33 & 8 & 7 & 9 & 9 & $2.66 \mathrm{E}-10$ & 12.6 \\
\hline Aldolase(41)-Aldolase(138) & Intra & 32 & 9 & 7 & 9 & 7 & $2.29 \mathrm{E}-12$ & 29.9 \\
\hline Aldolase(139)-Aldolase(329) & Intra & 30 & 7 & 5 & 10 & 8 & 2.05E-11 & 32.6 \\
\hline Aldolase(110)-Aldolase(110) & Inter & 26 & 3 & 8 & 11 & 4 & $3.11 \mathrm{E}-14$ & 16.3 \\
\hline Aldolase(317)-Aldolase(329) & Intra & 25 & 5 & 4 & 9 & 7 & $8.95 \mathrm{E}-15$ & 19.4 \\
\hline Aldolase(98)-Aldolase(139) & Intra & 25 & 9 & 3 & 6 & 7 & 5.00E-14 & 13.9 \\
\hline Aldolase(13)-Aldolase(139) & Intra & 19 & 3 & 3 & 7 & 6 & 3.57E-15 & 18.2 \\
\hline Aldolase(316)-Aldolase(329) & Intra & 19 & 2 & 8 & 6 & 3 & 3.50E-10 & 19.7 \\
\hline Aldolase(100)-Aldolase(329) & Intra & 18 & 3 & 4 & 6 & 5 & $3.72 \mathrm{E}-18$ & 16.8 \\
\hline Aldolase(13)-Aldolase(138) & Intra & 18 & - & 6 & 7 & 5 & $5.42 \mathrm{E}-10$ & 16.0 \\
\hline Aldolase(107)-Aldolase(138) & Intra & 12 & 2 & 4 & 4 & 2 & 3.59E-09 & 12.3 \\
\hline Aldolase(138)-Aldolase(329) & Intra & 12 & - & 5 & 3 & 4 & $1.26 \mathrm{E}-08$ & 30.8 \\
\hline Aldolase(98)-Aldolase(329) & Intra & 12 & - & 4 & 7 & 1 & $8.18 \mathrm{E}-07$ & 20.7 \\
\hline Aldolase(316)-Aldolase(321) & Intra & 12 & - & 5 & 4 & 3 & $2.60 \mathrm{E}-04$ & 10.5 \\
\hline Aldolase(139)-Aldolase(311) & Intra & 11 & 1 & 3 & 6 & 1 & 1.67E-12 & 34.7 \\
\hline Aldolase(41)-Aldolase(329) & Intra & 11 & 2 & 3 & 3 & 3 & $6.71 \mathrm{E}-11$ & 23.5 \\
\hline Aldolase(138)-Aldolase(139) & Intra & 11 & - & 4 & 6 & 1 & $9.67 \mathrm{E}-10$ & 3.8 \\
\hline Aldolase(41)-Aldolase(139) & Intra & 10 & 5 & 2 & 2 & 1 & 5.52E-11 & 31.6 \\
\hline Aldolase(139)-Aldolase(316) & Intra & 9 & 1 & 3 & 3 & 2 & $1.55 \mathrm{E}-08$ & 34.3 \\
\hline Aldolase(100)-Aldolase(139) & Intra & 8 & - & 3 & 3 & 2 & $3.34 \mathrm{E}-14$ & 19.1 \\
\hline Aldolase(107)-Aldolase(146) & Intra & 8 & 1 & 3 & 2 & 2 & $3.14 \mathrm{E}-08$ & 4.4 \\
\hline Aldolase(98)-Aldolase(317) & Intra & 7 & 1 & 5 & 1 & - & $5.85 \mathrm{E}-09$ & 24.4 \\
\hline Aldolase(13)-Aldolase(329) & Intra & 6 & - & 3 & 3 & - & $3.56 \mathrm{E}-17$ & 33.9 \\
\hline Aldolase(139)-Aldolase(139) & Inter & 6 & 2 & 1 & 2 & 1 & $1.62 \mathrm{E}-05$ & 40.3 \\
\hline Aldolase(138)-Aldolase(146) & Intra & 5 & - & 1 & 2 & 2 & $2.34 \mathrm{E}-13$ & 13.3 \\
\hline Aldolase(107)-Aldolase(110) & Intra & 5 & - & 2 & 3 & - & $3.59 \mathrm{E}-10$ & 8.6 \\
\hline Aldolase(138)-Aldolase(321) & Intra & 5 & - & 1 & 1 & 3 & 2.00E-05 & 32.0 \\
\hline bsa(211)-bsa(242) & Intra & 144 & 47 & 32 & 35 & 30 & $1.40 \mathrm{E}-18$ & 10.5 \\
\hline bsa(520)-bsa(524) & Intra & 138 & 18 & 21 & 48 & 51 & $2.22 \mathrm{E}-18$ & 6.5 \\
\hline bsa(4)-bsa(12) & Intra & 117 & 30 & 32 & 26 & 29 & $9.80 \mathrm{E}-14$ & 12.6 \\
\hline bsa(211)-bsa(239) & Intra & 88 & 27 & 27 & 17 & 17 & $3.03 E-12$ & 9.5 \\
\hline bsa(204)-bsa(465) & Intra & 60 & 15 & 15 & 15 & 15 & 2.23E-10 & 13.5 \\
\hline bsa(431)-bsa(439) & Intra & 53 & 17 & 12 & 11 & 13 & 3.31E-09 & 13.4 \\
\hline bsa(413)-bsa(537) & Intra & 51 & 10 & 13 & 13 & 15 & $7.69 \mathrm{E}-15$ & 11.7 \\
\hline bsa(221)-bsa(294) & Intra & 48 & 12 & 11 & 14 & 11 & $6.19 \mathrm{E}-06$ & 4.2 \\
\hline bsa(159)-bsa(180) & Intra & 41 & 12 & 17 & 5 & 7 & 1.16E-09 & 10.0 \\
\hline bsa(12)-bsa(51) & Intra & 40 & 14 & 11 & 8 & 7 & $1.84 \mathrm{E}-12$ & 9.3 \\
\hline bsa(280)-bsa(294) & Intra & 40 & 12 & 8 & 10 & 10 & $1.21 \mathrm{E}-05$ & 18.4 \\
\hline bsa(211)-bsa(350) & Intra & 39 & 11 & 10 & 11 & 7 & $8.51 \mathrm{E}-12$ & 13.5 \\
\hline
\end{tabular}




\begin{tabular}{|c|c|c|c|c|c|c|c|c|}
\hline bsa(116)-bsa(431) & Intra & 35 & 11 & 9 & 8 & 7 & $3.13 \mathrm{E}-04$ & 21.2 \\
\hline bsa(396)-bsa(544) & Intra & 33 & 13 & 14 & 4 & 2 & $5.32 \mathrm{E}-27$ & 14.5 \\
\hline bsa(204)-bsa(242) & Intra & 32 & 5 & 4 & 15 & 8 & $9.60 \mathrm{E}-09$ & 12.6 \\
\hline bsa(204)-bsa(350) & Intra & 30 & 11 & 8 & 7 & 4 & $3.32 \mathrm{E}-10$ & 16.9 \\
\hline bsa(211)-bsa(322) & Intra & 28 & 8 & 3 & 9 & 8 & $6.91 \mathrm{E}-11$ & 14.4 \\
\hline bsa(294)-bsa(316) & Intra & 23 & 8 & 5 & 5 & 5 & $5.61 \mathrm{E}-12$ & 33.7 \\
\hline bsa(20)-bsa(132) & Intra & 22 & 5 & 8 & 6 & 3 & $9.53 \mathrm{E}-10$ & 11.5 \\
\hline bsa(273)-bsa(280) & Intra & 21 & 2 & 7 & 7 & 5 & $8.92 \mathrm{E}-12$ & 13.7 \\
\hline bsa(41)-bsa(132) & Intra & 21 & 10 & 8 & 1 & 2 & $4.70 \mathrm{E}-06$ & 12.6 \\
\hline bsa(127)-bsa(132) & Intra & 18 & 1 & 2 & 6 & 9 & $9.23 \mathrm{E}-15$ & 10.4 \\
\hline bsa(93)-bsa(106) & Intra & 16 & - & 2 & 10 & 4 & $5.32 \mathrm{E}-08$ & 17.1 \\
\hline bsa(221)-bsa(239) & Intra & 14 & 4 & 2 & 4 & 4 & $2.71 \mathrm{E}-07$ & 19.4 \\
\hline bsa(106)-bsa(116) & Intra & 14 & 4 & 1 & 8 & 1 & $1.77 \mathrm{E}-04$ & 24.0 \\
\hline bsa(362)-bsa(377) & Intra & 13 & 1 & 2 & 5 & 5 & $2.63 \mathrm{E}-06$ & 23.2 \\
\hline bsa(64)-bsa(465) & Intra & 12 & - & - & 5 & 7 & $2.41 \mathrm{E}-07$ & 32.4 \\
\hline bsa(294)-bsa(439) & Intra & 10 & 2 & 5 & 2 & 1 & $1.16 \mathrm{E}-07$ & 17.7 \\
\hline bsa(221)-bsa(439) & Intra & 10 & 2 & 6 & - & 2 & $2.88 \mathrm{E}-05$ & 19.9 \\
\hline bsa(322)-bsa(350) & Intra & 9 & 3 & 5 & 1 & - & $1.90 \mathrm{E}-21$ & 12.4 \\
\hline bsa(524)-bsa(544) & Intra & 9 & 5 & 4 & - & - & $1.49 \mathrm{E}-16$ & 13.3 \\
\hline bsa(413)-bsa(465) & Intra & 9 & - & 1 & 4 & 4 & $7.13 \mathrm{E}-15$ & 19.6 \\
\hline bsa(12)-bsa(132) & Intra & 9 & 3 & 2 & 1 & 3 & $6.02 \mathrm{E}-07$ & 22.6 \\
\hline bsa(322)-bsa(362) & Intra & 8 & 3 & 3 & 2 & - & $1.61 \mathrm{E}-08$ & 17.6 \\
\hline bsa(211)-bsa(362) & Intra & 7 & 3 & 3 & - & 1 & $1.82 \mathrm{E}-06$ & 30.0 \\
\hline bsa(204)-bsa(211) & Intra & 6 & 1 & 1 & 1 & 3 & $4.50 \mathrm{E}-15$ & 13.7 \\
\hline bsa(204)-bsa(439) & Intra & 6 & - & - & 3 & 3 & $4.78 \mathrm{E}-13$ & 33.0 \\
\hline bsa(221)-bsa(242) & Intra & 6 & - & - & 4 & 2 & $1.97 \mathrm{E}-05$ & 21.4 \\
\hline bsa(544)-bsa(563) & Inter & 5 & 1 & - & 1 & 3 & $4.67 \mathrm{E}-09$ & 22.2 \\
\hline bsa(211)-bsa(439) & Intra & 5 & 1 & - & 3 & 1 & $2.00 \mathrm{E}-08$ & 29.1 \\
\hline bsa(471)-bsa(537) & Intra & 5 & 1 & - & 3 & 1 & $8.89 \mathrm{E}-07$ & 19.1 \\
\hline bsa(12)-bsa(12) & Inter & 5 & 1 & 3 & - & 1 & $5.00 \mathrm{E}-06$ & 57.7 \\
\hline bsa(93)-bsa(114) & Intra & 5 & - & - & 3 & 2 & $2.16 \mathrm{E}-03$ & 34.9 \\
\hline Cbf5(87)-Cbf5(114) & Intra & 95 & 20 & 16 & 28 & 31 & $5.63 \mathrm{E}-12$ & 14.7 \\
\hline Cbf5(134)-Cbf5(180) & Intra & 91 & 24 & 23 & 23 & 21 & $6.95 \mathrm{E}-15$ & 11.0 \\
\hline Cbf5(114)-Cbf5(337) & Intra & 83 & 11 & 36 & 19 & 17 & $9.16 \mathrm{E}-14$ & 12.2 \\
\hline Cbf5(161)-Gar1(115) & Inter & 79 & 17 & 24 & 19 & 19 & $5.56 \mathrm{E}-09$ & 18.9 \\
\hline Gar1(104)-Gar1(115) & Intra & 65 & - & 4 & 27 & 34 & $5.22 \mathrm{E}-12$ & 24.7 \\
\hline Cbf5(180)-Nop10(19) & Inter & 62 & 20 & 24 & 9 & 9 & $1.94 \mathrm{E}-13$ & 15.5 \\
\hline Nop10(1)-Nop10(19) & Intra & 61 & 19 & 12 & 15 & 15 & $1.60 \mathrm{E}-20$ & 11.5 \\
\hline Cbf5(180)-Nop10(18) & Inter & 59 & 14 & 16 & 16 & 13 & $2.99 \mathrm{E}-08$ & 13.0 \\
\hline Cbf5(31)-Cbf5(267) & Intra & 57 & 19 & 18 & 10 & 10 & $5.07 \mathrm{E}-2$ & 4 \\
\hline
\end{tabular}




\begin{tabular}{|c|c|c|c|c|c|c|c|c|}
\hline Cbf5(114)-Cbf5(161) & Intra & 45 & 9 & 10 & 12 & 14 & $1.19 \mathrm{E}-09$ & 24.0 \\
\hline Gar1(77)-Gar1(115) & Intra & 38 & 7 & 17 & 4 & 10 & $3.63 \mathrm{E}-18$ & 11.0 \\
\hline Cbf5(80)-Cbf5(87) & Intra & 16 & - & - & 9 & 7 & $6.94 \mathrm{E}-08$ & 7.0 \\
\hline Cbf5(161)-Gar1(77) & Inter & 16 & 6 & 10 & - & - & $4.99 \mathrm{E}-06$ & 18.9 \\
\hline Nop10(28)-Nop10(40) & Intra & 13 & 1 & 6 & 3 & 3 & $1.02 \mathrm{E}-04$ & 24.4 \\
\hline Nhp2(61)-Nop10(40) & Inter & 68 & 12 & 20 & 19 & 17 & $4.95 \mathrm{E}-09$ & na \\
\hline Nop10(40)-Nop10(49) & Intra & 38 & 4 & 3 & 15 & 16 & $8.61 \mathrm{E}-11$ & 32.6[Nop10(40)-Nop10(48)] \\
\hline Nhp2(69)-Nop10(40) & Inter & 29 & 9 & 7 & 7 & 6 & $3.06 \mathrm{E}-06$ & na \\
\hline Nhp2(65)-Nop10(40) & Inter & 27 & 4 & 8 & 7 & 8 & $1.64 \mathrm{E}-12$ & na \\
\hline Nhp2(107)-Nop10(19) & Inter & 26 & 2 & 6 & 10 & 8 & $1.26 \mathrm{E}-07$ & na \\
\hline Nhp2(54)-Nhp2(138) & Intra & 24 & 3 & 3 & 8 & 10 & $3.56 \mathrm{E}-07$ & $\sim 15.6$ \\
\hline Nhp2(65)-Nhp2(69) & Intra & 24 & 4 & 3 & 8 & 9 & $6.17 \mathrm{E}-06$ & $\sim 5.8$ \\
\hline Nhp2(69)-Nhp2(131) & Intra & 23 & 5 & 4 & 6 & 8 & $2.16 \mathrm{E}-05$ & $\sim 11.8$ \\
\hline Cbf5(50)-Cbf5(383) & Intra & 22 & 3 & 6 & 9 & 4 & $4.78 \mathrm{E}-06$ & $\sim 27.8[\mathrm{Cbf5}(50)-\mathrm{Cbf5}(350)]$ \\
\hline Cbf5(363)-Cbf5(370) & Intra & 19 & 3 & 2 & 6 & 8 & $1.34 \mathrm{E}-06$ & na \\
\hline Cbf5(358)-Cbf5(360) & Intra & 17 & - & - & 8 & 9 & 4.99E-09 & na \\
\hline Cbf5(50)-Cbf5(370) & Intra & 14 & - & 3 & 6 & 5 & $8.90 \mathrm{E}-08$ & $\sim 27.8[\mathrm{Cbf} 5(50)-\mathrm{Cbf} 5(350)]$ \\
\hline Nhp2(45)-Nhp2(49) & Intra & 13 & 2 & 2 & 5 & 4 & 8.77E-06 & $\sim 6.1$ \\
\hline Nhp2(54)-Nhp2(131) & Intra & 12 & - & - & 5 & 7 & $2.73 \mathrm{E}-06$ & $\sim 17.5$ \\
\hline Cbf5(114)-Cbf5(383) & Intra & 11 & 2 & 3 & 3 & 3 & $1.24 \mathrm{E}-09$ & 33.3[Cbf5(114)-Cbf5(350)] \\
\hline Nop10(28)-Nop10(49) & Intra & 11 & - & - & 6 & 5 & $2.02 \mathrm{E}-05$ & 34.8[Nop10(28)-Nop10(48)] \\
\hline Nhp2(133)-Nhp2(143) & Intra & 9 & - & - & 4 & 5 & $1.83 \mathrm{E}-03$ & $\sim 16.4$ \\
\hline Cbf5(50)-Cbf5(358) & Intra & 8 & - & - & 3 & 5 & $3.44 \mathrm{E}-05$ & $\sim 27.8[\mathrm{Cbf5}(50)-\mathrm{Cbf} 5(350)]$ \\
\hline Cbf5(370)-Cbf5(383) & Intra & 7 & - & - & 4 & 3 & $3.72 \mathrm{E}-07$ & na \\
\hline Nhp2(57)-Nhp2(65) & Intra & 7 & - & - & 4 & 3 & $1.66 \mathrm{E}-05$ & $\sim 10.4$ \\
\hline Nhp2(131)-Nhp2(133) & Intra & 7 & - & 2 & 1 & 4 & $2.16 \mathrm{E}-04$ & $\sim 5.9$ \\
\hline Nhp2(38)-Nhp2(45) & Intra & 7 & 1 & - & 3 & 3 & $1.52 \mathrm{E}-03$ & $\sim 11.4$ \\
\hline Cbf5(358)-Cbf5(370) & Intra & 6 & - & - & 3 & 3 & $4.83 \mathrm{E}-09$ & na \\
\hline Cbf5(114)-Cbf5(370) & Intra & 6 & 3 & 2 & 1 & - & $4.41 \mathrm{E}-06$ & $\sim 33.3[\mathrm{Cbf5}(114)-\mathrm{Cbf5}(350)]$ \\
\hline Nhp2(15)-Nhp2(45) & Intra & 6 & - & - & 3 & 3 & $2.92 \mathrm{E}-03$ & 32.6[Nhp2(27)-Nhp2(45)] \\
\hline Nhp2(15)-Nop10(19) & Inter & 5 & 3 & - & 2 & - & $1.06 \mathrm{E}-12$ & na \\
\hline Nhp2(15)-Nhp2(41) & Intra & 5 & - & - & 3 & 2 & $1.73 \mathrm{E}-04$ & 28.0[Nhp2(27)-Nhp2(41)] \\
\hline Cbf5(50)-Cbf5(360) & Intra & 5 & - & - & 3 & 2 & $1.89 \mathrm{E}-03$ & 27.8[Cbf5(50)-Cbf5(350)] \\
\hline Nhp2(131)-Nhp2(143) & Intra & 4 & - & - & 2 & 2 & $2.72 \mathrm{E}-09$ & $\sim 15.6$ \\
\hline \multicolumn{9}{|c|}{$\mathrm{BS}^{3}$} \\
\hline Site-pairs & Inter/Intra & $\begin{array}{c}\text { \#total } \\
\text { spec }\end{array}$ & \#Exp1 & \#Exp2 & \#Ехр3 & \#Exp4 & Best E_value & Ca-Ca distance \\
\hline Aldolase(41)-Aldolase(311) & Intra & 699 & 126 & 148 & 191 & 234 & $9.5 \mathrm{E}-14$ & 12.7 \\
\hline Aldolase(1)-Aldolase(1) & Inter & 194 & 42 & 51 & 58 & 43 & $1.3 \mathrm{E}-19$ & 10.1 \\
\hline Aldolase(12)-Aldolase(199) & Inter & 95 & 22 & 28 & 23 & 22 & $5.8 \mathrm{E}-15$ & 15.2 \\
\hline
\end{tabular}




\begin{tabular}{|c|c|c|c|c|c|c|c|c|}
\hline Aldolase(98)-Aldolase(139) & Intra & 77 & 12 & 25 & 26 & 14 & 1.7E-17 & 13.9 \\
\hline Aldolase(98)-Aldolase(138) & Intra & 68 & 17 & 19 & 15 & 17 & $1.3 \mathrm{E}-15$ & 13.4 \\
\hline Aldolase(13)-Aldolase(199) & Inter & 64 & 14 & 19 & 13 & 18 & $9.9 \mathrm{E}-18$ & 17.2 \\
\hline Aldolase(1)-Aldolase(139) & Intra & 62 & 14 & 22 & 10 & 16 & $1.0 \mathrm{E}-18$ & 34.6 \\
\hline Aldolase(1)-Aldolase(207) & Inter & 53 & 16 & 11 & 12 & 14 & $2.2 \mathrm{E}-18$ & 7.8 \\
\hline Aldolase(1)-Aldolase(199) & Inter & 52 & 12 & 12 & 16 & 12 & $2.3 \mathrm{E}-14$ & 12.3 \\
\hline Aldolase(1)-Aldolase(41) & Inter & 47 & 16 & 7 & 14 & 10 & $5.0 \mathrm{E}-07$ & 33.0 \\
\hline Aldolase(86)-Aldolase(139) & Intra & 43 & 7 & 16 & 7 & 13 & $7.6 \mathrm{E}-12$ & 16.5 \\
\hline Aldolase(13)-Aldolase(138) & Intra & 40 & 12 & 9 & 11 & 8 & $2.9 \mathrm{E}-11$ & 16.0 \\
\hline Aldolase(317)-Aldolase(329) & Intra & 39 & 10 & 10 & 9 & 10 & $1.7 \mathrm{E}-13$ & 19.4 \\
\hline Aldolase(110)-Aldolase(110) & Inter & 36 & 6 & 15 & 6 & 9 & 4.4E-15 & 16.3 \\
\hline Aldolase(100)-Aldolase(321) & Intra & 35 & 11 & 5 & 11 & 8 & $2.3 \mathrm{E}-12$ & 17.7 \\
\hline Aldolase(13)-Aldolase(139) & Intra & 33 & 9 & 12 & 5 & 7 & $1.9 \mathrm{E}-18$ & 18.2 \\
\hline Aldolase(311)-Aldolase(329) & Intra & 30 & 7 & 6 & 10 & 7 & $3.1 \mathrm{E}-09$ & 13.9 \\
\hline Aldolase(316)-Aldolase(321) & Intra & 30 & 8 & 10 & 6 & 6 & 7.7E-05 & 10.5 \\
\hline Aldolase(146)-Aldolase(152) & Intra & 28 & 9 & 6 & 7 & 6 & $2.3 \mathrm{E}-10$ & 19.6 \\
\hline Aldolase(13)-Aldolase(329) & Intra & 24 & 5 & 8 & 3 & 8 & $6.7 \mathrm{E}-18$ & 33.9 \\
\hline Aldolase(311)-Aldolase(317) & Intra & 23 & 4 & 5 & 7 & 7 & $3.9 \mathrm{E}-10$ & 12.6 \\
\hline Aldolase(199)-Aldolase(207) & Intra & 20 & 4 & 8 & 4 & 4 & 3.7E-16 & 12.4 \\
\hline Aldolase(139)-Aldolase(321) & Intra & 19 & 3 & 5 & 6 & 5 & 5.2E-19 & 32.5 \\
\hline Aldolase(138)-Aldolase(139) & Intra & 19 & 8 & 5 & 3 & 3 & $9.9 \mathrm{k}-09$ & 3.8 \\
\hline Aldolase(41)-Aldolase(138) & Intra & 18 & 6 & f & 4 & 1 & $4.9 \mathrm{E}-13$ & 29.9 \\
\hline Aldolase(41)-Aldolase(107) & Intra & 14 & 3 & - & 11 & - & $6.6 \mathrm{E}-10$ & 18.9 \\
\hline Aldolase(138)-Aldolase(321) & Intra & 14 & 8 & - & 5 & 1 & $3.0 \mathrm{E}-08$ & 32.0 \\
\hline Aldolase(1)-Aldolase(138) & Intra & 10 & 3 & 3 & 2 & 2 & $2.0 \mathrm{E}-06$ & 32.0 \\
\hline Aldolase(12)-Aldolase(139) & Intra & 9 & 4 & 2 & 1 & 2 & $1.0 \mathrm{E}-15$ & 18.1 \\
\hline Aldolase(13)-Aldolase(98) & Intra & 9 & 6 & - & 2 & 1 & $1.1 \mathrm{E}-10$ & 19.6 \\
\hline Aldolase(139)-Aldolase(329) & Intra & 6 & 1 & 1 & 3 & 1 & 7.4E-15 & 32.6 \\
\hline Aldolase(107)-Aldolase(110) & Intra & 6 & 1 & 3 & - & 2 & $2.1 \mathrm{E}-12$ & 8.6 \\
\hline Aldolase(13)-Aldolase(146) & Intra & 6 & 1 & 4 & 1 & - & 8.1E-12 & 20.1 \\
\hline Aldolase(139)-Aldolase(139) & Inter & 6 & - & 4 & - & 2 & $1.4 \mathrm{E}-10$ & 40.3 \\
\hline Aldolase(138)-Aldolase(146) & Intra & 6 & - & 1 & - & 5 & $3.0 \mathrm{E}-10$ & 13.3 \\
\hline Aldolase(139)-Aldolase(317) & Intra & 6 & - & 4 & - & 2 & $1.2 \mathrm{E}-09$ & 32.5 \\
\hline Aldolase(138)-Aldolase(329) & Intra & 6 & 1 & 3 & 2 & - & $2.4 \mathrm{E}-07$ & 30.8 \\
\hline Aldolase(13)-Aldolase(207) & Inter & 5 & 3 & - & 2 & - & $3.8 \mathrm{E}-17$ & 21.6 \\
\hline Aldolase(139)-Aldolase(311) & Intra & 5 & 2 & - & 1 & 2 & $2.3 \mathrm{E}-09$ & 34.7 \\
\hline Aldolase(100)-Aldolase(138) & Intra & 5 & 2 & - & 3 & - & $8.6 \mathrm{E}-08$ & 18.5 \\
\hline Aldolase(138)-Aldolase(317) & Intra & 4 & 1 & - & 3 & - & $2.5 \mathrm{E}-10$ & 32.3 \\
\hline Aldolase(12)-Aldolase(138) & Intra & 4 & 2 & - & - & 2 & $8.2 \mathrm{E}-06$ & 15.6 \\
\hline bsa(211 & Intra & 399 & 83 & 91 & 128 & 97 & $2.4 \mathrm{E}-13$ & 3.5 \\
\hline
\end{tabular}




\begin{tabular}{|c|c|c|c|c|c|c|c|c|}
\hline bsa(520)-bsa(524) & Intra & 196 & 14 & 19 & 92 & 71 & $1.2 \mathrm{E}-20$ & 6.5 \\
\hline bsa(4)-bsa(12) & Intra & 165 & 46 & 40 & 41 & 38 & $4.1 \mathrm{E}-15$ & 12.6 \\
\hline bsa(221)-bsa(439) & Intra & 84 & 19 & 22 & 24 & 19 & $4.1 \mathrm{E}-08$ & 19.9 \\
\hline bsa(159)-bsa(180) & Intra & 81 & 20 & 28 & 16 & 17 & $1.2 \mathrm{E}-10$ & 10.0 \\
\hline bsa(211)-bsa(242) & Intra & 77 & 18 & 15 & 26 & 18 & $1.9 \mathrm{E}-18$ & 10.5 \\
\hline bsa(211)-bsa(239) & Intra & 77 & 18 & 23 & 19 & 17 & $6.0 \mathrm{E}-13$ & 9.5 \\
\hline bsa(294)-bsa(439) & Intra & 67 & 14 & 18 & 18 & 17 & $6.4 \mathrm{E}-16$ & 17.7 \\
\hline bsa(211)-bsa(362) & Intra & 67 & 17 & 2 & 33 & 15 & $3.5 \mathrm{E}-11$ & 30.0 \\
\hline bsa(431)-bsa(439) & Intra & 65 & 22 & 16 & 12 & 15 & $6.2 \mathrm{E}-09$ & 13.4 \\
\hline bsa(204)-bsa(465) & Intra & 63 & 15 & 15 & 17 & 16 & $1.3 \mathrm{E}-11$ & 13.5 \\
\hline bsa(413)-bsa(537) & Intra & 60 & 9 & 19 & 18 & 14 & $2.6 \mathrm{E}-14$ & 11.7 \\
\hline bsa(396)-bsa(544) & Intra & 53 & 22 & 20 & 6 & 5 & $1.2 \mathrm{E}-21$ & 14.5 \\
\hline bsa(204)-bsa(474) & Intra & 50 & 11 & 13 & 13 & 13 & $5.2 \mathrm{E}-16$ & 13.1 \\
\hline bsa(20)-bsa(132) & Intra & 47 & 10 & 14 & 13 & 10 & $1.3 \mathrm{E}-08$ & 11.5 \\
\hline bsa(204)-bsa(242) & Intra & 45 & 4 & 9 & 16 & 16 & $6.3 \mathrm{E}-09$ & 12.6 \\
\hline bsa(12)-bsa(51) & Intra & 41 & 8 & 7 & 15 & 11 & $2.6 \mathrm{E}-15$ & 9.3 \\
\hline bsa(294)-bsa(316) & Intra & 39 & 9 & 11 & 10 & 9 & $3.6 \mathrm{E}-11$ & 33.7 \\
\hline bsa(211)-bsa(322) & Intra & 37 & 8 & 9 & 10 & 10 & 3.1E-11 & 14.4 \\
\hline bsa(280)-bsa(294) & Intra & 36 & 14 & 9 & 6 & 7 & $4.5 \mathrm{E}-07$ & 18.4 \\
\hline bsa(204)-bsa(350) & Intra & 33 & 10 & 10 & 7 & 6 & $4.2 \mathrm{E}-10$ & 16.9 \\
\hline bsa(204)-bsa(431) & Intra & 30 & 9 & 10 & 4 & 7 & $2.0 \mathrm{E}-08$ & 26.9 \\
\hline bsa(204)-bsa(211) & Intra & 28 & 9 & 9 & 5 & 5 & 4.1E-14 & 13.7 \\
\hline bsa(524)-bsa(544) & Intra & 27 & 7 & 10 & 6 & 4 & $1.8 \mathrm{E}-15$ & 13.3 \\
\hline bsa(413)-bsa(465) & Intra & 27 & 2 & 8 & 11 & 6 & $3.8 \mathrm{E}-12$ & 19.6 \\
\hline bsa(221)-bsa(239) & Intra & 26 & 7 & 12 & 3 & 4 & $1.4 \mathrm{E}-09$ & 19.4 \\
\hline bsa(12)-bsa(12) & Inter & 23 & - & 2 & 14 & 7 & $2.8 \mathrm{E}-07$ & 57.7 \\
\hline bsa(12)-bsa(261) & Intra & 22 & 9 & 5 & 5 & 3 & $1.8 \mathrm{E}-17$ & 16.4 \\
\hline bsa(242)-bsa(465) & Intra & 22 & - & - & 14 & 8 & $3.0 \mathrm{E}-13$ & 21.6 \\
\hline bsa(159)-bsa(280) & Intra & 20 & 11 & 7 & 1 & 1 & 4.4E-08 & 14.2 \\
\hline bsa(350)-bsa(474) & Intra & 17 & 9 & 5 & 1 & 2 & $2.0 \mathrm{E}-21$ & 17.9 \\
\hline bsa(116)-bsa(431) & Intra & 17 & 3 & 5 & 6 & 3 & $1.9 \mathrm{E}-04$ & 21.2 \\
\hline bsa(273)-bsa(280) & Intra & 15 & - & 3 & 6 & 6 & $8.1 \mathrm{E}-13$ & 13.7 \\
\hline bsa(41)-bsa(132) & Intra & 15 & 9 & 5 & 1 & - & $1.2 \mathrm{E}-07$ & 12.6 \\
\hline bsa(465)-bsa(474) & Intra & 14 & 4 & 2 & 3 & 5 & $1.8 \mathrm{E}-20$ & 14.5 \\
\hline bsa(471)-bsa(537) & Intra & 14 & - & 4 & 5 & 5 & 7.4E-08 & 19.1 \\
\hline bsa(221)-bsa(232) & Intra & 14 & 3 & 5 & 3 & 3 & $4.0 \mathrm{E}-04$ & 14.8 \\
\hline bsa(12)-bsa(132) & Intra & 11 & 4 & 2 & 2 & 3 & $4.2 \mathrm{E}-08$ & 22.6 \\
\hline bsa(211)-bsa(221) & Intra & 11 & 5 & 2 & 2 & 2 & $1.6 \mathrm{E}-07$ & 15.1 \\
\hline bsa(159)-bsa(275) & Intra & 10 & 4 & 5 & 1 & - & $8.3 \mathrm{E}-08$ & 21.7 \\
\hline bsa(4)-bsa(64) & Intra & 9 & - & - & 3 & 6 & $2.5 \mathrm{E}-10$ & 9.6 \\
\hline
\end{tabular}




\begin{tabular}{|c|c|c|c|c|c|c|c|c|}
\hline bsa(204)-bsa(471) & Intra & 9 & 6 & 2 & 1 & - & $1.2 \mathrm{E}-07$ & 17.1 \\
\hline bsa(221)-bsa(294) & Intra & 9 & 5 & 3 & - & 1 & $1.5 \mathrm{E}-05$ & 4.2 \\
\hline bsa(64)-bsa(465) & Intra & 8 & - & - & 4 & 4 & $1.4 \mathrm{E}-10$ & 32.4 \\
\hline bsa(204)-bsa(221) & Intra & 8 & 4 & 4 & - & - & $3.8 \mathrm{E}-07$ & 26.5 \\
\hline bsa(93)-bsa(106) & Intra & 8 & - & 6 & 2 & - & $9.1 \mathrm{E}-07$ & 17.1 \\
\hline bsa(180)-bsa(431) & Intra & 8 & 3 & 3 & 1 & 1 & $2.1 \mathrm{E}-06$ & 19.5 \\
\hline bsa(322)-bsa(350) & Intra & 7 & 4 & 3 & - & - & $1.1 \mathrm{E}-22$ & 12.4 \\
\hline bsa(413)-bsa(474) & Intra & 7 & 3 & 4 & - & - & $3.8 \mathrm{E}-20$ & 13.0 \\
\hline bsa(211)-bsa(465) & Intra & 7 & - & - & 4 & 3 & $1.7 \mathrm{E}-09$ & 26.0 \\
\hline bsa(211)-bsa(232) & Intra & 7 & 2 & 1 & 3 & 1 & $1.7 \mathrm{E}-08$ & 10.8 \\
\hline bsa(504)-bsa(524) & Intra & 5 & 1 & - & 3 & 1 & $5.2 \mathrm{E}-19$ & 13.6 \\
\hline bsa(204)-bsa(439) & Intra & 5 & 2 & 1 & 1 & 1 & $6.7 \mathrm{E}-13$ & 33.0 \\
\hline bsa(180)-bsa(439) & Intra & 5 & 3 & 1 & 1 & - & $5.5 \mathrm{E}-08$ & 22.1 \\
\hline bsa(221)-bsa(242) & Intra & 5 & - & 1 & 1 & 3 & $2.1 \mathrm{E}-07$ & 21.4 \\
\hline bsa(413)-bsa(471) & Intra & 5 & 3 & 2 & - & - & $4.9 \mathrm{E}-07$ & 8.7 \\
\hline bsa(439)-bsa(537) & Intra & 5 & - & - & 3 & 2 & $9.1 \mathrm{E}-05$ & 35.2 \\
\hline bsa(114)-bsa(431) & Intra & 5 & 3 & 2 & - & - & $1.2 \mathrm{E}-03$ & 19.2 \\
\hline bsa(51)-bsa(76) & Intra & 5 & 3 & 2 & - & - & $1.5 \mathrm{E}-03$ & 0.3 \\
\hline bsa(439)-bsa(465) & Intra & 4 & - & - & 2 & 2 & $2.9 \mathrm{E}-08$ & 35.7 \\
\hline Cbf5(134)-Cbf5(180) & Intra & 114 & 39 & 38 & 25 & 12 & $4.2 \mathrm{E}-15$ & 11.0 \\
\hline Nop10(1)-Nop10(19) & Intra & 92 & 22 & 24 & 26 & 20 & 7.3E-17 & 11.5 \\
\hline Cbf5(161)-Gar1(115) & Inter & 69 & 11 & 19 & 21 & 18 & $2.6 \mathrm{E}-07$ & 18.9 \\
\hline Cbf5(180)-Nop10(19) & Inter & 54 & 15 & 16 & 18 & 5 & $1.4 \mathrm{E}-14$ & 15.5 \\
\hline Cbf5(31)-Cbf5(267) & Intra & 47 & 11 & 18 & 10 & 8 & $2.3 \mathrm{E}-22$ & 11.4 \\
\hline Cbf5(114)-Cbf5(161) & Intra & 35 & 9 & 10 & 11 & 5 & $3.8 \mathrm{E}-09$ & 24.0 \\
\hline Cbf5(9)-Cbf5(50) & Intra & 33 & 2 & 7 & 14 & 10 & $5.5 \mathrm{E}-09$ & 16.6 \\
\hline Cbf5(161)-Gar1(77) & Inter & 24 & 8 & 9 & 5 & 2 & $2.0 \mathrm{E}-06$ & 18.9 \\
\hline Gar1(77)-Gar1(115) & Intra & 20 & 5 & 10 & 2 & 3 & $4.2 \mathrm{E}-19$ & 11.0 \\
\hline Cbf5(161)-Gar1(59) & Inter & 19 & 4 & 8 & 6 & 1 & $2.0 \mathrm{E}-07$ & 22.0 \\
\hline Cbf5(50)-Cbf5(267) & Intra & 18 & 8 & 8 & 1 & 1 & 4.7E-07 & 23.0 \\
\hline Gar1(59)-Nop10(40) & Inter & 17 & 4 & 5 & 5 & 3 & $4.5 \mathrm{E}-06$ & 59.6 \\
\hline Cbf5(180)-Nop10(18) & Inter & 15 & 4 & 4 & 4 & 3 & $1.2 \mathrm{E}-06$ & 13.0 \\
\hline Cbf5(114)-Nop10(40) & Inter & 14 & 6 & 5 & 2 & 1 & $1.3 \mathrm{E}-08$ & 35.0 \\
\hline Nop10(19)-Nop10(40) & Intra & 14 & 3 & 4 & 4 & 3 & $6.9 \mathrm{E}-06$ & 30.6 \\
\hline Cbf5(161)-Nop10(40) & Inter & 14 & 4 & 6 & 4 & - & $7.2 \mathrm{E}-06$ & 44.1 \\
\hline Cbf5(50)-Cbf5(161) & Intra & 11 & 3 & 2 & 3 & 3 & $2.6 \mathrm{E}-05$ & 49.2 \\
\hline Cbf5(87)-Cbf5(114) & Intra & 9 & - & - & 4 & 5 & $1.1 \mathrm{E}-09$ & 14.7 \\
\hline Gar1(104)-Gar1(115) & Intra & 8 & - & - & 5 & 3 & $5.6 \mathrm{E}-07$ & 24.7 \\
\hline Cbf5(180)-Nop10(40) & Inter & 6 & 3 & 2 & 1 & - & $3.3 \mathrm{E}-11$ & 41.8 \\
\hline Cbf5(114)-Cbf5(337) & Intra & 6 & - & 1 & 2 & 3 & $1.1 \mathrm{E}-10$ & 12. \\
\hline
\end{tabular}




\begin{tabular}{|c|c|c|c|c|c|c|c|c|}
\hline Nop10(1)-Nop10(40) & Intra & 6 & - & - & 4 & 2 & $2.0 \mathrm{E}-06$ & 25.1 \\
\hline Cbf5(134)-Nop10(18) & Inter & 6 & - & - & 3 & 3 & $3.9 \mathrm{E}-05$ & 21.9 \\
\hline Cbf5(50)-Gar1(59) & Inter & 4 & - & 3 & 1 & - & $2.1 \mathrm{E}-07$ & 58.3 \\
\hline Nhp2(61)-Nop10(40) & Inter & 46 & 11 & 14 & 15 & 6 & $1.4 \mathrm{E}-09$ & na \\
\hline Nhp2(69)-Nop10(40) & Inter & 26 & 4 & 8 & 10 & 4 & 1.7E-05 & na \\
\hline Nhp2(65)-Nop10(40) & Inter & 20 & 6 & 2 & 8 & 4 & $2.3 \mathrm{E}-11$ & na \\
\hline Nhp2(65)-Nhp2(69) & Intra & 17 & 2 & 3 & 7 & 5 & $1.6 \mathrm{E}-07$ & $\sim 5.8$ \\
\hline Nop10(40)-Nop10(40) & Inter & 13 & 2 & 4 & 5 & 2 & $1.4 \mathrm{E}-09$ & na \\
\hline Gar1(59)-Gar1(59) & Inter & 10 & - & 5 & 4 & 1 & $3.9 \mathrm{E}-13$ & na \\
\hline Nhp2(45)-Nhp2(49) & Intra & 7 & - & - & 4 & 3 & 2.7E-05 & $\sim 6.1$ \\
\hline Cbf5(114)-Nhp2(69) & Inter & 4 & - & - & 3 & 1 & $1.1 \mathrm{E}-04$ & na \\
\hline \multicolumn{9}{|c|}{ DSS } \\
\hline Site-pairs & Inter/Intra & $\begin{array}{c}\text { \#total } \\
\text { spec }\end{array}$ & \#Exp1 & \#Exp2 & \#Еxp3 & \#Exp4 & Best E_value & Ca-Ca distance \\
\hline Aldolase(41)-Aldolase(311) & Intra & 645 & 54 & 160 & 205 & 226 & $1.10 \mathrm{E}-16$ & 12.7 \\
\hline Aldolase(12)-Aldolase(199) & Inter & 152 & 25 & 28 & 41 & 58 & $1.35 \mathrm{E}-16$ & 15.2 \\
\hline Aldolase(98)-Aldolase(139) & Intra & 98 & 50 & 15 & 13 & 20 & $1.70 \mathrm{E}-16$ & 13.9 \\
\hline Aldolase(86)-Aldolase(139) & Intra & 75 & 15 & 16 & 21 & 23 & $1.78 \mathrm{E}-11$ & 16.5 \\
\hline Aldolase(1)-Aldolase(1) & Inter & 64 & 24 & - & 19 & 21 & $2.12 \mathrm{E}-14$ & 10.1 \\
\hline Aldolase(98)-Aldolase(138) & Intra & 57 & 7 & 16 & 20 & 14 & $3.62 \mathrm{E}-14$ & 13.4 \\
\hline Aldolase(110)-Aldolase(110) & Inter & 54 & 9 & 13 & 17 & 15 & $2.05 E-16$ & 16.3 \\
\hline Aldolase(100)-Aldolase(321) & Intra & 46 & 12 & 12 & 13 & 9 & $5.41 \mathrm{E}-11$ & 17.7 \\
\hline Aldolase(311)-Aldolase(329) & Intra & 41 & 11 & 10 & 15 & 5 & $2.48 \mathrm{E}-09$ & 13.9 \\
\hline Aldolase(316)-Aldolase(321) & Intra & 37 & 6 & 12 & 11 & 8 & $8.72 \mathrm{E}-05$ & 10.5 \\
\hline Aldolase(12)-Aldolase(139) & Intra & 33 & 4 & 11 & 9 & 9 & $7.12 \mathrm{E}-17$ & 18.1 \\
\hline Aldolase(139)-Aldolase(321) & Intra & 27 & 3 & 8 & 8 & 8 & $1.93 \mathrm{E}-21$ & 32.5 \\
\hline Aldolase(317)-Aldolase(329) & Intra & 27 & 3 & 8 & 8 & 8 & $1.50 \mathrm{E}-12$ & 19.4 \\
\hline Aldolase(311)-Aldolase(317) & Intra & 27 & 3 & 8 & 8 & 8 & 4.13E-10 & 12.6 \\
\hline Aldolase(41)-Aldolase(138) & Intra & 25 & 8 & 5 & 6 & 6 & $7.54 \mathrm{E}-12$ & 29.9 \\
\hline Aldolase(107)-Aldolase(110) & Intra & 23 & 5 & 6 & 7 & 5 & $9.43 \mathrm{E}-16$ & 8.6 \\
\hline Aldolase(12)-Aldolase(138) & Intra & 21 & - & 4 & 10 & 7 & $1.00 \mathrm{E}-10$ & 15.6 \\
\hline Aldolase(100)-Aldolase(138) & Intra & 20 & 4 & 4 & 5 & 7 & $4.38 \mathrm{E}-11$ & 18.5 \\
\hline Aldolase(316)-Aldolase(329) & Intra & 19 & - & 3 & 10 & 6 & $4.95 \mathrm{E}-14$ & 19.7 \\
\hline Aldolase(12)-Aldolase(207) & Inter & 19 & 4 & 5 & 6 & 4 & $1.94 \mathrm{E}-13$ & 18.5 \\
\hline Aldolase(1)-Aldolase(139) & Intra & 19 & 5 & 2 & 5 & 7 & $2.20 \mathrm{E}-11$ & 34.6 \\
\hline Aldolase(139)-Aldolase(317) & Intra & 19 & - & 6 & 6 & 7 & $8.93 E-11$ & 32.5 \\
\hline Aldolase(41)-Aldolase(139) & Intra & 18 & 6 & 4 & 4 & 4 & $6.09 \mathrm{E}-19$ & 31.6 \\
\hline Aldolase(146)-Aldolase(152) & Intra & 18 & 6 & 5 & 4 & 3 & $3.64 \mathrm{E}-10$ & 19.6 \\
\hline Aldolase(139)-Aldolase(329) & Intra & 17 & 4 & 7 & 3 & 3 & $3.78 \mathrm{E}-15$ & 32.6 \\
\hline Aldolase(139)-Aldolase(139) & Inter & 17 & 5 & 4 & 3 & 5 & 2.47E-09 & 40.3 \\
\hline
\end{tabular}




\begin{tabular}{|c|c|c|c|c|c|c|c|c|}
\hline Aldolase(100)-Aldolase(139) & Intra & 16 & 3 & 4 & 3 & 6 & $1.03 \mathrm{E}-14$ & 19.1 \\
\hline Aldolase(199)-Aldolase(207) & Intra & 15 & 5 & 3 & 3 & 4 & $7.27 \mathrm{E}-17$ & 12.4 \\
\hline Aldolase(138)-Aldolase(139) & Intra & 14 & - & 4 & 4 & 6 & 7.57E-09 & 3.8 \\
\hline Aldolase(12)-Aldolase(329) & Intra & 12 & - & 2 & 7 & 3 & $3.45 \mathrm{E}-10$ & 35.4 \\
\hline Aldolase(27)-Aldolase(100) & Intra & 12 & 3 & 4 & 3 & 2 & $6.81 \mathrm{E}-09$ & 12.4 \\
\hline Aldolase(139)-Aldolase(311) & Intra & 10 & - & 6 & 4 & - & $3.57 \mathrm{E}-12$ & 34.7 \\
\hline Aldolase(107)-Aldolase(321) & Intra & 10 & 2 & - & 4 & 4 & $1.22 \mathrm{E}-06$ & 27.7 \\
\hline Aldolase(41)-Aldolase(152) & Intra & 9 & 5 & 2 & 1 & 1 & $1.26 \mathrm{E}-06$ & 22.3 \\
\hline Aldolase(100)-Aldolase(317) & Intra & 8 & - & 4 & - & 4 & $4.24 \mathrm{E}-10$ & 22.5 \\
\hline Aldolase(138)-Aldolase(321) & Intra & 8 & - & 2 & 2 & 4 & $2.03 \mathrm{E}-08$ & 32.0 \\
\hline Aldolase(13)-Aldolase(138) & Intra & 7 & 3 & 1 & 1 & 2 & $1.16 \mathrm{E}-10$ & 16.0 \\
\hline Aldolase(138)-Aldolase(329) & Intra & 7 & - & - & 3 & 4 & $3.55 \mathrm{E}-07$ & 30.8 \\
\hline Aldolase(86)-Aldolase(317) & Intra & 7 & 6 & 1 & - & - & $9.81 \mathrm{E}-04$ & 16.7 \\
\hline Aldolase(100)-Aldolase(329) & Intra & 6 & 3 & - & 3 & - & 1.09E-13 & 16.8 \\
\hline Aldolase(100)-Aldolase(146) & Intra & 6 & - & 2 & 2 & 2 & $1.34 \mathrm{E}-12$ & 20.7 \\
\hline Aldolase(1)-Aldolase(138) & Intra & 6 & - & 1 & 1 & 4 & $9.12 \mathrm{E}-04$ & 32.0 \\
\hline Aldolase(41)-Aldolase(317) & Intra & 5 & 2 & - & 3 & - & $2.42 \mathrm{E}-10$ & 19.3 \\
\hline Aldolase(138)-Aldolase(317) & Intra & 5 & - & 1 & 3 & 1 & $1.16 \mathrm{E}-08$ & 32.3 \\
\hline Aldolase(13)-Aldolase(199) & Inter & 4 & - & - & 3 & 1 & $1.73 \mathrm{E}-13$ & 17.2 \\
\hline Aldolase(13)-Aldolase(139) & Intra & 4 & - & 2 & - & 2 & $1.65 \mathrm{E}-06$ & 18.2 \\
\hline Aldolase(316)-Aldolase(317) & Intra & 4 & - & - & 2 & 2 & $9.32 \mathrm{E}-06$ & 3.8 \\
\hline bsa(211)-bsa(350) & Intra & 514 & 87 & 134 & 153 & 140 & $1.51 \mathrm{E}-14$ & 13.5 \\
\hline bsa(520)-bsa(524) & Intra & 285 & 51 & 64 & 96 & 74 & $3.47 \mathrm{E}-20$ & 6.5 \\
\hline bsa(211)-bsa(362) & Intra & 149 & 49 & 37 & 35 & 28 & $3.05 \mathrm{E}-10$ & 30.0 \\
\hline bsa(4)-bsa(12) & Intra & 140 & 30 & 27 & 43 & 40 & $6.79 \mathrm{E}-14$ & 12.6 \\
\hline bsa(211)-bsa(242) & Intra & 119 & 30 & 22 & 33 & 34 & $2.47 \mathrm{E}-18$ & 10.5 \\
\hline bsa(211)-bsa(239) & Intra & 105 & 29 & 28 & 26 & 22 & $4.87 \mathrm{E}-13$ & 9.5 \\
\hline bsa(20)-bsa(132) & Intra & 103 & 31 & 31 & 21 & 20 & $1.97 \mathrm{E}-13$ & 11.5 \\
\hline bsa(221)-bsa(439) & Intra & 80 & 18 & 21 & 21 & 20 & $2.12 \mathrm{E}-08$ & 19.9 \\
\hline bsa(159)-bsa(180) & Intra & 79 & 15 & 17 & 24 & 23 & $9.12 \mathrm{E}-11$ & 10.0 \\
\hline bsa(204)-bsa(242) & Intra & 75 & 8 & 13 & 27 & 27 & $4.86 \mathrm{E}-11$ & 12.6 \\
\hline bsa(294)-bsa(439) & Intra & 71 & 14 & 20 & 19 & 18 & $1.80 \mathrm{E}-11$ & 17.7 \\
\hline bsa(431)-bsa(439) & Intra & 62 & 19 & 13 & 16 & 14 & $1.95 \mathrm{E}-11$ & 13.4 \\
\hline bsa(204)-bsa(465) & Intra & 61 & 15 & 15 & 15 & 16 & $1.58 \mathrm{E}-11$ & 13.5 \\
\hline bsa(413)-bsa(537) & Intra & 54 & 7 & 17 & 16 & 14 & $2.18 \mathrm{E}-20$ & 11.7 \\
\hline bsa(4)-bsa(64) & Intra & 54 & 15 & 10 & 17 & 12 & $1.25 \mathrm{E}-10$ & 9.6 \\
\hline bsa(12)-bsa(51) & Intra & 50 & 12 & 13 & 12 & 13 & $5.53 \mathrm{E}-17$ & 9.3 \\
\hline bsa(396)-bsa(544) & Intra & 47 & 20 & 17 & 5 & 5 & $3.88 \mathrm{E}-28$ & 14.5 \\
\hline bsa(204)-bsa(474) & Intra & 40 & 9 & 6 & 13 & 12 & $8.47 \mathrm{E}-17$ & 13.1 \\
\hline bsa(204)-bsa(350) & Intra & 39 & 9 & 10 & 11 & 9 & $1.96 \mathrm{E}-10$ & 16.9 \\
\hline
\end{tabular}




\begin{tabular}{|c|c|c|c|c|c|c|c|c|}
\hline bsa(280)-bsa(294) & Intra & 39 & 11 & 9 & 7 & 12 & $8.06 E-07$ & 18.4 \\
\hline bsa(211)-bsa(322) & Intra & 36 & 8 & 9 & 10 & 9 & 8.49E-11 & 14.4 \\
\hline bsa(350)-bsa(474) & Intra & 35 & 9 & 12 & 9 & 5 & $1.19 \mathrm{E}-23$ & 17.9 \\
\hline bsa(221)-bsa(239) & Intra & 35 & 14 & 8 & 8 & 5 & $1.95 \mathrm{E}-09$ & 19.4 \\
\hline bsa(224)-bsa(275) & Intra & 33 & 7 & 13 & 5 & 8 & 4.70E-17 & 15.3 \\
\hline bsa(350)-bsa(375) & Intra & 29 & 10 & 12 & 4 & 3 & $9.06 E-14$ & 12.1 \\
\hline bsa(524)-bsa(544) & Intra & 28 & 9 & 10 & 7 & 2 & $1.13 E-17$ & 13.3 \\
\hline bsa(294)-bsa(316) & Intra & 26 & 6 & 7 & 5 & 8 & $2.28 \mathrm{E}-11$ & 33.7 \\
\hline bsa(12)-bsa(132) & Intra & 25 & 10 & 6 & 5 & 4 & $2.99 \mathrm{E}-13$ & 22.6 \\
\hline bsa(273)-bsa(280) & Intra & 25 & 2 & 5 & 11 & 7 & $9.02 \mathrm{E}-10$ & 13.7 \\
\hline bsa(221)-bsa(294) & Intra & 25 & 3 & 8 & 10 & 4 & $7.04 \mathrm{E}-06$ & 4.2 \\
\hline bsa(221)-bsa(242) & Intra & 24 & 6 & 7 & 7 & 4 & $3.14 \mathrm{E}-07$ & 21.4 \\
\hline bsa(180)-bsa(439) & Intra & 23 & - & - & 14 & 9 & $1.32 \mathrm{E}-14$ & 22.1 \\
\hline bsa(180)-bsa(431) & Intra & 22 & - & - & 12 & 10 & $3.66 \mathrm{E}-07$ & 19.5 \\
\hline bsa(204)-bsa(431) & Intra & 21 & 2 & 5 & 7 & 7 & $3.33 \mathrm{E}-09$ & 26.9 \\
\hline bsa(413)-bsa(465) & Intra & 20 & - & - & 10 & 10 & $2.92 \mathrm{E}-12$ & 19.6 \\
\hline bsa(159)-bsa(280) & Intra & 20 & 10 & 7 & 1 & 2 & $1.56 \mathrm{E}-07$ & 14.2 \\
\hline bsa(204)-bsa(211) & Intra & 17 & 3 & 4 & 5 & 5 & $1.38 \mathrm{E}-14$ & 13.7 \\
\hline bsa(242)-bsa(465) & Intra & 17 & - & - & 6 & 11 & $1.56 \mathrm{E}-14$ & 21.6 \\
\hline bsa(211)-bsa(232) & Intra & 17 & 3 & 2 & 5 & 7 & $2.15 \mathrm{E}-09$ & 10.8 \\
\hline bsa(4)-bsa(431) & Intra & 16 & - & - & 9 & 7 & $3.12 \mathrm{E}-08$ & 49.8 \\
\hline bsa(4)-bsa(204) & Intra & 15 & - & - & 8 & 7 & $1.77 \mathrm{E}-11$ & 33.3 \\
\hline bsa(322)-bsa(350) & Intra & 14 & 4 & 8 & 1 & 1 & 8.19E-31 & 12.4 \\
\hline bsa(504)-bsa(524) & Intra & 12 & 6 & 5 & - & 1 & 4.49E-18 & 13.6 \\
\hline bsa(413)-bsa(431) & Intra & 12 & - & - & 5 & 7 & 7.76E-07 & 19.5 \\
\hline bsa(413)-bsa(471) & Intra & 11 & - & - & 4 & 7 & $1.87 \mathrm{E}-08$ & 8.7 \\
\hline bsa(211)-bsa(221) & Intra & 11 & 3 & 2 & 3 & 3 & $2.19 \mathrm{E}-08$ & 15.1 \\
\hline bsa(93)-bsa(106) & Intra & 11 & - & 5 & 3 & 3 & 7.83E-06 & 17.1 \\
\hline bsa(116)-bsa(431) & Intra & 11 & 5 & 6 & - & - & $6.72 \mathrm{E}-05$ & 21.2 \\
\hline bsa(221)-bsa(232) & Intra & 11 & 3 & 3 & 3 & 2 & $2.01 \mathrm{E}-04$ & 14.8 \\
\hline bsa(322)-bsa(362) & Intra & 10 & 6 & 3 & - & 1 & $2.04 \mathrm{E}-08$ & 17.6 \\
\hline bsa(41)-bsa(132) & Intra & 10 & 8 & 2 & - & - & $1.03 E-06$ & 12.6 \\
\hline bsa(159)-bsa(275) & Intra & 10 & 4 & 5 & 1 & - & $1.67 \mathrm{E}-06$ & 21.7 \\
\hline bsa(64)-bsa(465) & Intra & 9 & - & - & 4 & 5 & 1.79E-11 & 32.4 \\
\hline bsa(204)-bsa(221) & Intra & 9 & 5 & 4 & - & - & $4.18 \mathrm{E}-06$ & 26.5 \\
\hline bsa(465)-bsa(474) & Intra & 8 & - & - & 4 & 4 & $3.14 \mathrm{E}-16$ & 14.5 \\
\hline bsa(12)-bsa(12) & Inter & 8 & - & 1 & 5 & 2 & $2.46 \mathrm{E}-06$ & 57.7 \\
\hline bsa(431)-bsa(465) & Intra & 8 & - & - & 4 & 4 & $1.85 \mathrm{E}-03$ & 25.0 \\
\hline bsa(127)-bsa(132) & Intra & 7 & 3 & 4 & - & - & 4.16E-10 & 10.4 \\
\hline bsa(362)-bsa(474) & Intra & 7 & 2 & 2 & 1 & 2 & $6.81 \mathrm{E}-10$ & 30.7 \\
\hline
\end{tabular}




\begin{tabular}{|c|c|c|c|c|c|c|c|c|}
\hline bsa(224)-bsa(280) & Intra & 7 & 6 & - & - & 1 & $1.49 \mathrm{E}-08$ & 22.8 \\
\hline bsa(93)-bsa(465) & Intra & 7 & - & - & 4 & 3 & $1.22 \mathrm{E}-07$ & 22.2 \\
\hline bsa(64)-bsa(471) & Intra & 6 & - & - & 3 & 3 & $1.23 \mathrm{E}-06$ & 44.2 \\
\hline bsa(431)-bsa(537) & Intra & 6 & - & - & 3 & 3 & $9.29 \mathrm{E}-05$ & 26.1 \\
\hline bsa(204)-bsa(439) & Intra & 5 & - & - & 4 & 1 & $1.02 \mathrm{E}-12$ & 33.0 \\
\hline bsa(431)-bsa(471) & Intra & 5 & - & - & 2 & 3 & $1.04 \mathrm{E}-08$ & 23.8 \\
\hline bsa(204)-bsa(322) & Intra & 5 & - & - & 2 & 3 & $1.58 \mathrm{E}-06$ & 21.4 \\
\hline bsa(4)-bsa(242) & Intra & 5 & - & 1 & 4 & - & $1.67 \mathrm{E}-06$ & 22.5 \\
\hline bsa(114)-bsa(431) & Intra & 5 & 3 & 2 & - & - & $2.84 \mathrm{E}-05$ & 19.2 \\
\hline bsa(273)-bsa(294) & Intra & 5 & - & 3 & - & 2 & $3.30 \mathrm{E}-04$ & 7.2 \\
\hline bsa(12)-bsa(64) & Intra & 4 & - & - & 2 & 2 & $1.58 \mathrm{E}-13$ & 16.4 \\
\hline bsa(4)-bsa(439) & Intra & 4 & - & - & 1 & 3 & 7.34E-11 & 52.5 \\
\hline bsa(204)-bsa(362) & Intra & 4 & - & - & 3 & 1 & $1.33 \mathrm{E}-07$ & 32.3 \\
\hline bsa(471)-bsa(537) & Intra & 4 & - & 3 & - & 1 & $4.02 \mathrm{E}-06$ & 19.1 \\
\hline bsa(413)-bsa(535) & Intra & 4 & 1 & - & 3 & - & $1.27 \mathrm{E}-03$ & 13.5 \\
\hline Cbf5(134)-Cbf5(180) & Intra & 115 & 27 & 41 & 22 & 25 & 7.96E-16 & 11.0 \\
\hline Cbf5(114)-Cbf5(337) & Intra & 104 & 14 & 18 & 27 & 45 & $3.17 \mathrm{E}-13$ & 12.2 \\
\hline Nop10(1)-Nop10(19) & Intra & 102 & 20 & 25 & 26 & 31 & $6.15 E-18$ & L.5 \\
\hline Cbf5(9)-Cbf5(50) & Intra & 97 & 29 & 32 & 18 & 18 & $4.34 \mathrm{E}-12$ & 16.6 \\
\hline Cbf5(161)-Gar1(115) & Inter & 91 & 19 & 23 & 23 & 26 & $5.22 \mathrm{E}-09$ & 18.9 \\
\hline Cbf5(87)-Cbf5(114) & Intra & 88 & 13 & 12 & 29 & 34 & $1.43 \mathrm{E}-13$ & 14.7 \\
\hline Cbf5(180)-Nop10(19) & Inter & 72 & 12 & 23 & 16 & 21 & $1.99 \mathrm{E}-14$ & 15.5 \\
\hline Gar1(77)-Gar1(115) & Intra & 67 & 7 & 23 & 18 & 19 & 1.19E-19 & 11.0 \\
\hline Cbf5(180)-Nop10(18) & Inter & 59 & 11 & 20 & 12 & 16 & $7.86 \mathrm{E}-07$ & 13.0 \\
\hline Cbf5(114)-Cbf5(161) & Intra & 52 & 13 & 15 & 11 & 13 & $5.16 \mathrm{E}-09$ & 24.0 \\
\hline Cbf5(31)-Cbf5(267) & Intra & 39 & 10 & 9 & 10 & 10 & $1.83 \mathrm{E}-20$ & 11.4 \\
\hline Cbf5(50)-Cbf5(267) & Intra & 39 & 8 & 7 & 11 & 13 & $3.56 \mathrm{E}-06$ & 23.0 \\
\hline Cbf5(161)-Gar1(59) & Inter & 36 & 10 & 9 & 8 & 9 & $5.81 \mathrm{E}-07$ & 22.0 \\
\hline Cbf5(161)-Gar1(77) & Inter & 26 & 5 & 9 & 6 & 6 & $8.23 \mathrm{E}-10$ & 18.9 \\
\hline Cbf5(80)-Cbf5(87) & Intra & 25 & 7 & 6 & 3 & 9 & $1.34 \mathrm{E}-08$ & 7.0 \\
\hline Nop10(28)-Nop10(40) & Intra & 19 & 4 & 2 & 7 & 6 & $1.45 \mathrm{E}-06$ & 24.4 \\
\hline Cbf5(137)-Nop10(18) & Inter & 18 & 3 & 6 & 4 & 5 & $1.61 \mathrm{E}-07$ & 19.0 \\
\hline Gar1(104)-Gar1(115) & Intra & 17 & - & - & 10 & 7 & $1.31 \mathrm{E}-14$ & 24.7 \\
\hline Cbf5(114)-Nop10(40) & Inter & 17 & 4 & 5 & 4 & 4 & $3.44 \mathrm{E}-06$ & 35.0 \\
\hline Cbf5(54)-Cbf5(87) & Intra & 15 & 2 & 2 & 5 & 6 & $3.94 \mathrm{E}-08$ & 13.5 \\
\hline Nop10(19)-Nop10(40) & Intra & 15 & 2 & 3 & 4 & 6 & $8.00 \mathrm{E}-07$ & 30.6 \\
\hline Gar1(59)-Nop10(40) & Inter & 15 & 4 & 2 & 5 & 4 & $2.62 \mathrm{E}-06$ & 59.6 \\
\hline Cbł5(50)-Cbł5(309) & Intra & 11 & - & - & 6 & 5 & $3.00 \mathrm{E}-09$ & 9.9 \\
\hline Cbf5(134)-Nop10(18) & Inter & 11 & 3 & 3 & 3 & 2 & $2.41 \mathrm{E}-07$ & 21.9 \\
\hline Cbf5(134)-Nop10(40) & Inter & 11 & 1 & - & 4 & 6 & $1.93 \mathrm{E}-05$ & 51 \\
\hline
\end{tabular}




\begin{tabular}{|c|c|c|c|c|c|c|c|c|}
\hline Cbf5(161)-Nop10(40) & Inter & 11 & 5 & 2 & 1 & 3 & $1.66 \mathrm{E}-04$ & 44.1 \\
\hline Cbf5(27)-Cbf5(50) & Intra & 10 & - & 2 & 3 & 5 & $2.78 \mathrm{E}-07$ & 15.6 \\
\hline Cbf5(50)-Nop10(40) & Inter & 10 & - & 1 & 5 & 4 & $5.08 \mathrm{E}-06$ & 31.8 \\
\hline Cbf5(50)-Cbf5(114) & Intra & 9 & 6 & 3 & - & - & $4.31 \mathrm{E}-13$ & 26.4 \\
\hline Cbf5(50)-Cbf5(161) & Intra & 8 & 1 & - & 2 & 5 & 2.99E-04 & 49.2 \\
\hline Cbf5(9)-Cbf5(31) & Intra & 7 & 3 & 4 & - & - & $6.35 \mathrm{E}-05$ & 14.9 \\
\hline Cbf5(50)-Cbf5(134) & Intra & 5 & - & - & 2 & 3 & $6.66 \mathrm{E}-08$ & 60.2 \\
\hline Cbf5(121)-Cbf5(161) & Intra & 5 & 3 & 2 & - & - & $1.20 \mathrm{E}-05$ & 25.2 \\
\hline Cbf5(114)-Gar1(59) & Inter & 4 & 2 & 2 & - & - & $1.69 \mathrm{E}-09$ & 33.9 \\
\hline Cbf5(121)-Cbf5(137) & Intra & 4 & 2 & 2 & - & - & $2.96 \mathrm{E}-07$ & 26.2 \\
\hline Cbf5(80)-Cbf5(114) & Intra & 4 & 1 & - & 1 & 2 & $3.31 \mathrm{E}-06$ & 19.0 \\
\hline Nop10(1)-Nop10(40) & Intra & 4 & - & - & 1 & 3 & $1.66 \mathrm{E}-05$ & 25.1 \\
\hline Nop10(1)-Nop10(18) & Intra & 4 & - & 1 & 1 & 2 & $6.85 \mathrm{E}-05$ & 11.9 \\
\hline Nhp2(61)-Nop10(40) & Inter & 91 & 22 & 23 & 23 & 23 & $1.63 \mathrm{E}-10$ & na \\
\hline Nhp2(65)-Nop10(40) & Inter & 46 & 7 & 9 & 15 & 15 & $1.97 \mathrm{E}-14$ & na \\
\hline Nhp2(69)-Nhp2(131) & Intra & 42 & 10 & 12 & 10 & 10 & $9.60 \mathrm{E}-05$ & $\sim 11.8$ \\
\hline Nhp2(69)-Nop10(40) & Inter & 35 & 7 & 8 & 10 & 10 & $3.55 \mathrm{E}-06$ & na \\
\hline Nhp2(131)-Nhp2(143) & Intra & 34 & 8 & 9 & 9 & 8 & $1.91 \mathrm{E}-17$ & $\sim 15.6$ \\
\hline Nhp2(107)-Nop10(1) & Inter & 30 & 4 & 11 & 8 & 7 & $5.79 \mathrm{E}-14$ & na \\
\hline Cbf5(358)-Cbf5(360) & Intra & 29 & 6 & 7 & 7 & 9 & $9.69 \mathrm{E}-10$ & na \\
\hline Cbf5(114)-Cbf5(383) & Intra & 28 & 7 & 8 & 6 & 7 & $1.43 \mathrm{E}-11$ & 33.3[Cbf5(114)-Cbf5(350)] \\
\hline Nhp2(131)-Nhp2(133) & Intra & 27 & 4 & 7 & 8 & 8 & 9.77E-05 & $\sim 5.9$ \\
\hline Cbf5(370)-Cbf5(383) & Intra & 26 & 8 & 6 & 5 & 7 & $1.31 \mathrm{E}-10$ & na \\
\hline Nhp2(9)-Nhp2(15) & Intra & 26 & 8 & 7 & 5 & 6 & $1.53 \mathrm{E}-09$ & na \\
\hline Cbf5(363)-Cbf5(370) & Intra & 25 & 6 & 8 & 6 & 5 & $3.90 \mathrm{E}-07$ & na \\
\hline Nhp2(132)-Nhp2(143) & Intra & 24 & 6 & 9 & 5 & 4 & 2.63E-07 & $\sim 18.1$ \\
\hline Cbf5(50)-Cbf5(383) & Intra & 23 & 2 & 4 & 9 & 8 & $5.06 \mathrm{E}-10$ & 27.8[Cbf5(50)-Cbf5(350)] \\
\hline Cbf5(50)-Cbf5(370) & Intra & 22 & 3 & 6 & 5 & 8 & 1.39E-09 & $\sim 27.8[\mathrm{Cbf5}(50)-\mathrm{Cbf5}(350)]$ \\
\hline Nhp2(45)-Nhp2(49) & Intra & 22 & 6 & 6 & 4 & 6 & $1.64 \mathrm{E}-07$ & $\sim 6.1$ \\
\hline Nhp2(133)-Nhp2(143) & Intra & 22 & 5 & 5 & 6 & 6 & $7.16 \mathrm{E}-05$ & $\sim 16.4$ \\
\hline Nhp2(61)-Nhp2(69) & Intra & 21 & 3 & 5 & 6 & 7 & $6.41 \mathrm{E}-05$ & $\sim 13.6$ \\
\hline Nhp2(15)-Nop10(19) & Inter & 20 & 3 & 3 & 7 & 7 & $1.29 \mathrm{E}-16$ & na \\
\hline Nop10(40)-Nop10(49) & Intra & 20 & 2 & 3 & 6 & 9 & $3.58 \mathrm{E}-08$ & 32.6[Nop10(40)-Nop10(48)] \\
\hline Cbf5(114)-Cbf5(370) & Intra & 19 & 8 & 4 & 4 & 3 & $1.55 \mathrm{E}-08$ & 33.3[Cbf5(114)-Cbf5(350)] \\
\hline Nhp2(65)-Nhp2(69) & Intra & 19 & 2 & 2 & 8 & 7 & $2.51 E-05$ & $\sim 5.8$ \\
\hline Nhp2(54)-Nhp2(138) & Intra & 18 & 4 & 3 & 7 & 4 & $5.68 \mathrm{E}-06$ & $\sim 15.6$ \\
\hline Cbf5(358)-Cbf5(370) & Intra & 17 & 6 & 3 & 7 & 1 & $6.23 \mathrm{E}-11$ & na \\
\hline Nhp2(38)-Nhp2(45) & Intra & 17 & 4 & 5 & 3 & 5 & $3.76 \mathrm{E}-05$ & $\sim 11.4$ \\
\hline Nhp2(107)-Nop10(19) & Inter & 15 & 2 & 2 & 7 & 4 & 7.44E-07 & na \\
\hline Gar1(59)-Gar1(59) & Inter & 13 & 1 & 3 & 5 & 4 & $1.74 \mathrm{E}-11$ & na \\
\hline
\end{tabular}




\begin{tabular}{|c|c|c|c|c|c|c|c|c|}
\hline Nhp2(15)-Nhp2(41) & Intra & 13 & 3 & 5 & 3 & 2 & $2.40 \mathrm{E}-07$ & $\sim 28.0[\mathrm{Nhp} 2(27)-\mathrm{Nhp} 2(41)]$ \\
\hline Nhp2(45)-Nhp2(52) & Intra & 13 & - & 6 & 3 & 4 & $1.04 \mathrm{E}-06$ & $\sim 11.0$ \\
\hline Cbf5(360)-Cbf5(370) & Intra & 12 & - & 1 & 7 & 4 & 3.75E-09 & na \\
\hline Nhp2(57)-Nhp2(65) & Intra & 12 & 2 & 4 & 4 & 2 & 2.87E-06 & $\sim 10.4$ \\
\hline Nhp2(54)-Nhp2(131) & Intra & 12 & 1 & 1 & 5 & 5 & $1.14 \mathrm{E}-05$ & $\sim 17.5$ \\
\hline Nhp2(15)-Nhp2(32) & Intra & 11 & 3 & 1 & 2 & 5 & $5.96 \mathrm{E}-14$ & 12.4[Nhp2(27)-Nhp2(32)] \\
\hline Cbf5(161)-Cbf5(383) & Intra & 11 & 3 & 2 & 3 & 3 & $8.39 \mathrm{E}-05$ & $\sim 54.4[\mathrm{Cbf5}(161)-\mathrm{Cbf5}(350)]$ \\
\hline Nhp2(15)-Nhp2(45) & Intra & 11 & 3 & 5 & 1 & 2 & $3.13 \mathrm{E}-04$ & 32.6[Nhp2(27)-Nhp2(45)] \\
\hline Nhp2(65)-Nhp2(131) & Intra & 10 & 1 & 3 & 3 & 3 & $1.76 \mathrm{E}-09$ & $\sim 17.0$ \\
\hline Nhp2(6)-Nhp2(15) & Intra & 10 & 1 & 6 & 1 & 2 & $5.59 E-08$ & na \\
\hline Nhp2(107)-Nop10(28) & Inter & 10 & 3 & 3 & 2 & 2 & $9.16 \mathrm{E}-06$ & na \\
\hline Nhp2(57)-Nhp2(132) & Intra & 10 & 3 & 5 & 2 & - & $1.73 \mathrm{E}-05$ & $\sim 23.8$ \\
\hline Nhp2(2)-Nhp2(12) & Intra & 10 & 5 & 5 & - & - & $3.26 \mathrm{E}-05$ & na \\
\hline Nhp2(32)-Nhp2(54) & Intra & 10 & - & - & 6 & 4 & $2.45 \mathrm{E}-04$ & $\sim 30.6$ \\
\hline Nop10(28)-Nop10(49) & Intra & 9 & - & - & 4 & 5 & $7.26 \mathrm{E}-07$ & 34.8[Nop10(28)-Nop10(48)] \\
\hline Cbf5(284)-Cbf5(383) & Intra & 9 & 3 & 1 & 2 & 3 & $3.60 \mathrm{E}-06$ & 16.9[Cbf5(284)-Cbf5(350)] \\
\hline $\mathrm{Cbf5}(80)-\mathrm{Cbf} 5(383)$ & Intra & 9 & 2 & - & 4 & 3 & $2.51 \mathrm{E}-04$ & $\sim 37.6[\mathrm{Cbf5}(80)-\mathrm{Cbf5}(350)]$ \\
\hline Cbf5(180)-Nhp2(15) & Inter & 8 & 2 & - & 5 & 1 & $5.48 \mathrm{E}-07$ & na \\
\hline Nhp2(41)-Nhp2(45) & Intra & 8 & 3 & 1 & - & 4 & $1.12 \mathrm{E}-06$ & $\sim 6.7$ \\
\hline Cbf5(114)-Cbf5(114) & Inter & 8 & 2 & 2 & 2 & 2 & $1.01 \mathrm{E}-05$ & na \\
\hline Nhp2(41)-Nhp2(48) & Intra & 8 & - & 2 & 3 & 3 & $1.29 \mathrm{E}-04$ & $\sim 11.4$ \\
\hline Nhp2(15)-Nhp2(48) & Intra & 8 & - & 2 & 3 & 3 & $3.00 \mathrm{E}-04$ & 35.0[Nhp2(27)-Nhp2(48)] \\
\hline Nhp2(57)-Nhp2(131) & Intra & 8 & 2 & 2 & 1 & 3 & $3.68 \mathrm{E}-04$ & $\sim 20.0$ \\
\hline Cbf5(50)-Cbf5(358) & Intra & 7 & - & 1 & 3 & 3 & $6.12 \mathrm{E}-08$ & $\sim 27.8[\mathrm{Cbf} 5(50)-\mathrm{Cbf5}(350)]$ \\
\hline Cbf5(114)-Nhp2(69) & Inter & 7 & - & 3 & 3 & 1 & 3.19E-04 & na \\
\hline Cbf5(134)-Nhp2(32) & Inter & 6 & - & - & 2 & 4 & $3.91 \mathrm{E}-11$ & na \\
\hline Nhp2(37)-Nhp2(41) & Intra & 6 & 2 & 3 & - & 1 & $1.51 \mathrm{E}-04$ & $\sim 6.2$ \\
\hline Cbf5(284)-Cbf5(358) & Intra & 5 & 1 & 2 & 2 & - & $1.25 \mathrm{E}-09$ & 16.9[Cbf5(284)-Cbf5(350)] \\
\hline Cbf5(360)-Cbf5(367) & Intra & 5 & - & - & 3 & 2 & $9.27 \mathrm{E}-09$ & na \\
\hline Nhp2(37)-Nhp2(45) & Intra & 5 & - & - & 2 & 3 & $7.43 \mathrm{E}-07$ & $\sim 12.8$ \\
\hline Nhp2(12)-Nop10(19) & Inter & 5 & 2 & - & 1 & 2 & $1.01 \mathrm{E}-05$ & na \\
\hline Cbf5(137)-Nhp2(6) & Inter & 5 & 2 & 3 & - & - & $1.46 \mathrm{E}-05$ & na \\
\hline Nhp2(54)-Nhp2(132) & Intra & 5 & - & - & 3 & 2 & $1.16 \mathrm{E}-04$ & $\sim 21.2$ \\
\hline Cbf5(137)-Nhp2(69) & Inter & 5 & 1 & - & 4 & - & $1.80 \mathrm{E}-04$ & na \\
\hline Nhp2(6)-Nhp2(12) & Intra & 5 & 1 & 3 & - & 1 & $5.18 \mathrm{E}-04$ & na \\
\hline Cbf5(180)-Nhp2(12) & Inter & 4 & 1 & - & - & 3 & $2.19 \mathrm{E}-06$ & na \\
\hline Nhp2(117)-Nop10(19) & Inter & 4 & - & - & 2 & 2 & $2.05 \mathrm{E}-05$ & na \\
\hline \multirow[t]{2}{*}{ Nhp2(57)-Nhp2(61) } & Intra & 4 & - & 1 & 1 & 2 & $2.23 \mathrm{E}-03$ & $\sim 10.9$ \\
\hline & & & & EGS & & & & \\
\hline
\end{tabular}




\begin{tabular}{|c|c|c|c|c|c|c|c|c|}
\hline Site-pairs & Inter/Intra & $\begin{array}{c}\text { \#total } \\
\text { spec }\end{array}$ & \#Exp1 & \#Exp2 & \#Еxp3 & \#Exp4 & Best E_value & Ca-Ca distance \\
\hline Aldolase(98)-Aldolase(139) & Intra & 470 & 125 & 124 & 109 & 112 & $1.2 \mathrm{E}-20$ & 13.9 \\
\hline Aldolase(86)-Aldolase(139) & Intra & 255 & 51 & 53 & 69 & 82 & $2.2 \mathrm{E}-18$ & 16.5 \\
\hline Aldolase(41)-Aldolase(311) & Intra & 242 & 53 & 69 & 66 & 54 & 4.0E-25 & 12.7 \\
\hline Aldolase(110)-Aldolase(110) & Inter & 173 & 44 & 46 & 40 & 43 & $4.2 \mathrm{E}-19$ & 16.3 \\
\hline Aldolase(100)-Aldolase(321) & Intra & 124 & 30 & 32 & 34 & 28 & $3.9 \mathrm{E}-12$ & 17.7 \\
\hline Aldolase(41)-Aldolase(152) & Intra & 91 & 21 & 19 & 25 & 26 & $3.8 \mathrm{E}-14$ & 22.3 \\
\hline Aldolase(139)-Aldolase(139) & Inter & 72 & 18 & 15 & 20 & 19 & $8.2 \mathrm{E}-09$ & 40.3 \\
\hline Aldolase(139)-Aldolase(321) & Intra & 67 & 18 & 13 & 17 & 19 & $1.1 \mathrm{E}-15$ & 32.5 \\
\hline Aldolase(41)-Aldolase(107) & Intra & 60 & 15 & 18 & 14 & 13 & $8.3 \mathrm{E}-20$ & 18.9 \\
\hline Aldolase(12)-Aldolase(199) & Inter & 60 & 15 & 12 & 18 & 15 & 7.1E-16 & 15.2 \\
\hline Aldolase(41)-Aldolase(139) & Intra & 58 & 14 & 16 & 15 & 13 & $3.3 \mathrm{E}-24$ & 31.6 \\
\hline Aldolase(41)-Aldolase(329) & Intra & 42 & 7 & 11 & 13 & 11 & $3.9 \mathrm{E}-11$ & 23.5 \\
\hline Aldolase(139)-Aldolase(329) & Intra & 35 & 9 & 9 & 8 & 9 & $6.5 \mathrm{E}-17$ & 32.6 \\
\hline Aldolase(100)-Aldolase(329) & Intra & 30 & 5 & 6 & 11 & 8 & $1.2 \mathrm{E}-11$ & 16.8 \\
\hline Aldolase(139)-Aldolase(317) & Intra & 28 & 7 & 4 & 11 & 6 & $2.0 \mathrm{E}-13$ & 32.5 \\
\hline Aldolase(110)-Aldolase(138) & Intra & 28 & 8 & 8 & 6 & 6 & $1.1 \mathrm{E}-11$ & 18.2 \\
\hline Aldolase(311)-Aldolase(317) & Intra & 28 & - & - & 11 & 17 & $6.6 \mathrm{E}-09$ & 12.6 \\
\hline Aldolase(41)-Aldolase(100) & Intra & 26 & 4 & 10 & 7 & 5 & 4.0E-20 & 28.8 \\
\hline Aldolase(311)-Aldolase(329) & Intra & 26 & 9 & 8 & 5 & 4 & $2.8 \mathrm{E}-11$ & 13.9 \\
\hline Aldolase(41)-Aldolase(146) & Intra & 24 & 4 & 8 & 7 & 5 & $9.5 \mathrm{E}-16$ & 21.1 \\
\hline Aldolase(317)-Aldolase(329) & Intra & 22 & 6 & 2 & 7 & 7 & $9.8 \mathrm{E}-08$ & 19.4 \\
\hline Aldolase(139)-Aldolase(311) & Intra & 21 & 5 & 4 & 6 & 6 & $1.2 \mathrm{E}-17$ & 34.7 \\
\hline Aldolase(41)-Aldolase(110) & Intra & 19 & 5 & 6 & 5 & 3 & $2.8 \mathrm{E}-14$ & 18.9 \\
\hline Aldolase(329)-Aldolase(329) & Inter & 9 & 1 & 2 & 3 & 3 & $5.9 \mathrm{E}-15$ & 60.3 \\
\hline Aldolase(107)-Aldolase(138) & Intra & 6 & 2 & 1 & 3 & - & 3.0E-06 & 12.3 \\
\hline Aldolase(13)-Aldolase(199) & Inter & 6 & 1 & 1 & 2 & 2 & $1.0 \mathrm{E}-05$ & 17.2 \\
\hline Aldolase(138)-Aldolase(152) & Inter & 5 & 1 & 1 & 3 & - & $1.5 \mathrm{E}-05$ & 31.8 \\
\hline Aldolase(13)-Aldolase(329) & Intra & 4 & 1 & 1 & - & 2 & $2.3 \mathrm{E}-06$ & 33.9 \\
\hline bsa(211)-bsa(350) & Intra & 419 & 84 & 84 & 124 & 127 & $9.6 \mathrm{E}-13$ & 13.5 \\
\hline bsa(520)-bsa(524) & Intra & 369 & 68 & 82 & 102 & 117 & 7.5E-24 & 6.5 \\
\hline bsa(211)-bsa(242) & Intra & 360 & 110 & 99 & 83 & 68 & $7.0 \mathrm{E}-17$ & 10.5 \\
\hline bsa(431)-bsa(439) & Intra & 294 & 67 & 76 & 76 & 75 & $3.0 \mathrm{E}-12$ & 13.4 \\
\hline bsa(221)-bsa(439) & Intra & 205 & 54 & 46 & 54 & 51 & $8.0 \mathrm{E}-12$ & 19.9 \\
\hline bsa(204)-bsa(465) & Intra & 172 & 47 & 48 & 41 & 36 & $1.5 \mathrm{E}-18$ & 13.5 \\
\hline bsa(396)-bsa(413) & Intra & 157 & 40 & 40 & 40 & 37 & $1.8 \mathrm{E}-30$ & 22.4 \\
\hline bsa(211)-bsa(239) & Intra & 155 & 38 & 42 & 39 & 36 & $2.8 \mathrm{E}-13$ & 9.5 \\
\hline bsa(413)-bsa(537) & Intra & 146 & 36 & 33 & 39 & 38 & $3.5 \mathrm{E}-16$ & 11.7 \\
\hline bsa(396)-bsa(544) & Intra & 98 & 9 & 25 & 22 & 42 & $8.3 E-24$ & 14.5 \\
\hline
\end{tabular}




\begin{tabular}{|c|c|c|c|c|c|c|c|c|}
\hline bsa(4)-bsa(12) & Intra & 80 & 13 & 20 & 24 & 23 & 4.7E-13 & 12.6 \\
\hline bsa(204)-bsa(242) & Intra & 73 & 12 & 15 & 24 & 22 & $2.6 \mathrm{E}-15$ & 12.6 \\
\hline bsa(224)-bsa(273) & Intra & 68 & 19 & 15 & 18 & 16 & $5.8 \mathrm{E}-19$ & 10.9 \\
\hline bsa(377)-bsa(413) & Intra & 56 & 14 & 12 & 16 & 14 & $3.5 \mathrm{E}-23$ & 28.3 \\
\hline bsa(261)-bsa(285) & Intra & 54 & 8 & 11 & 17 & 18 & $5.6 \mathrm{E}-13$ & 9.2 \\
\hline bsa(224)-bsa(275) & Intra & 53 & 13 & 14 & 14 & 12 & 3.6E-19 & 15.3 \\
\hline bsa(431)-bsa(524) & Intra & 50 & 10 & 9 & 14 & 17 & $8.4 \mathrm{E}-08$ & 17.1 \\
\hline bsa(204)-bsa(474) & Intra & 47 & 17 & 11 & 10 & 9 & $4.3 \mathrm{E}-23$ & 13.1 \\
\hline bsa(114)-bsa(431) & Intra & 41 & 14 & 9 & 9 & 9 & $1.3 \mathrm{E}-05$ & 19.2 \\
\hline bsa(377)-bsa(474) & Intra & 40 & 13 & 9 & 10 & 8 & $2.9 \mathrm{E}-17$ & 26.0 \\
\hline bsa(524)-bsa(544) & Intra & 40 & 10 & 10 & 10 & 10 & $1.1 \mathrm{E}-13$ & 13.3 \\
\hline bsa(211)-bsa(232) & Intra & 39 & 5 & 9 & 14 & 11 & $5.5 \mathrm{E}-08$ & 10.8 \\
\hline bsa(93)-bsa(465) & Intra & 38 & 4 & 10 & 14 & 10 & $1.2 \mathrm{E}-24$ & 22.2 \\
\hline bsa(106)-bsa(116) & Intra & 34 & 8 & 10 & 8 & 8 & $1.5 \mathrm{E}-11$ & 24.0 \\
\hline bsa(211)-bsa(322) & Intra & 31 & 7 & 5 & 10 & 9 & $4.0 \mathrm{E}-07$ & 14.4 \\
\hline bsa(280)-bsa(294) & Intra & 28 & 7 & 5 & 9 & 7 & $1.1 \mathrm{E}-08$ & 18.4 \\
\hline bsa(12)-bsa(261) & Intra & 27 & 6 & 8 & 4 & 9 & $8.8 \mathrm{E}-16$ & 16.4 \\
\hline bsa(204)-bsa(211) & Intra & 24 & 4 & 4 & 8 & 8 & $2.5 \mathrm{E}-09$ & 13.7 \\
\hline bsa(221)-bsa(431) & Intra & 24 & 6 & 5 & 6 & 7 & 3.6E-08 & 25.9 \\
\hline bsa(93)-bsa(106) & Intra & 23 & 3 & 7 & 7 & 6 & $6.2 \mathrm{E}-12$ & 17.1 \\
\hline bsa(204)-bsa(413) & Intra & 23 & 3 & 6 & 5 & 9 & $1.7 \mathrm{E}-08$ & 23.8 \\
\hline bsa(159)-bsa(180) & Intra & 23 & 4 & 3 & 8 & 8 & $2.2 \mathrm{E}-08$ & 10.0 \\
\hline bsa(211)-bsa(413) & Intra & 23 & 2 & 2 & 9 & 10 & $4.5 \mathrm{E}-08$ & 32.0 \\
\hline bsa(211)-bsa(221) & Intra & 22 & 6 & 6 & 6 & 4 & $2.2 \mathrm{E}-07$ & 15.1 \\
\hline bsa(159)-bsa(280) & Intra & 17 & - & 6 & 6 & 5 & $9.0 \mathrm{E}-08$ & 14.2 \\
\hline bsa(204)-bsa(221) & Intra & 17 & 3 & 4 & 4 & 6 & $2.9 \mathrm{E}-07$ & 26.5 \\
\hline bsa(187)-bsa(439) & Intra & 17 & 5 & 10 & 1 & 1 & $4.5 \mathrm{E}-07$ & 17.5 \\
\hline bsa(180)-bsa(431) & Intra & 17 & 5 & 3 & 4 & 5 & $6.3 \mathrm{E}-07$ & 19.5 \\
\hline bsa(12)-bsa(51) & Intra & 16 & 1 & 5 & 5 & 5 & $3.8 \mathrm{E}-13$ & 9.3 \\
\hline bsa(51)-bsa(132) & Intra & 16 & 2 & 2 & 8 & 4 & $3.5 \mathrm{E}-08$ & 21.0 \\
\hline bsa(239)-bsa(242) & Intra & 15 & 2 & 4 & 5 & 4 & $1.5 \mathrm{E}-14$ & 5.0 \\
\hline bsa(204)-bsa(431) & Intra & 15 & 3 & 4 & 2 & 6 & $2.8 \mathrm{E}-09$ & 26.9 \\
\hline bsa(76)-bsa(413) & Intra & 13 & 4 & 2 & 4 & 3 & 1.7E-11 & 46.4 \\
\hline bsa(20)-bsa(132) & Intra & 13 & 3 & 2 & 7 & 1 & 1.7E-09 & 11.5 \\
\hline bsa(413)-bsa(474) & Intra & 11 & 1 & 5 & 2 & 3 & $1.0 \mathrm{E}-15$ & 13.0 \\
\hline bsa(114)-bsa(127) & Intra & 10 & - & 2 & 2 & 6 & $1.1 \mathrm{E}-09$ & 23.7 \\
\hline bsa(180)-bsa(280) & Intra & 10 & - & 3 & 2 & 5 & $5.8 \mathrm{E}-09$ & 21.8 \\
\hline bsa(116)-bsa(136) & Intra & 9 & - & - & 4 & 5 & 7.3E-13 & 14.4 \\
\hline bsa(232)-bsa(377) & Intra & 9 & - & - & 4 & 5 & $4.8 \mathrm{E}-12$ & 30.2 \\
\hline bsa(413)-bsa(431) & Intra & 8 & 1 & 3 & 2 & 2 & 6.6E-07 & 19.5 \\
\hline
\end{tabular}




\begin{tabular}{|c|c|c|c|c|c|c|c|c|}
\hline bsa(294)-bsa(439) & Intra & 7 & 4 & - & 2 & 1 & $5.1 \mathrm{E}-12$ & 17.7 \\
\hline bsa(51)-bsa(76) & Intra & 7 & - & - & 4 & 3 & $1.1 \mathrm{E}-08$ & 20.3 \\
\hline bsa(413)-bsa(439) & Intra & 7 & - & - & 3 & 4 & $2.0 \mathrm{E}-07$ & 28.7 \\
\hline bsa(413)-bsa(471) & Intra & 7 & 2 & 2 & 1 & 2 & $2.3 \mathrm{E}-06$ & 8.7 \\
\hline bsa(204)-bsa(350) & Intra & 6 & - & 2 & 1 & 3 & $2.2 \mathrm{E}-11$ & 16.9 \\
\hline bsa(64)-bsa(413) & Intra & 5 & - & - & 4 & 1 & 4.0E-16 & 50.0 \\
\hline bsa(261)-bsa(280) & Intra & 5 & 1 & 2 & 1 & 1 & $1.5 \mathrm{E}-04$ & 11.2 \\
\hline bsa(377)-bsa(396) & Intra & 4 & - & - & 1 & 3 & $1.2 \mathrm{E}-10$ & 30.0 \\
\hline Cbf5(134)-Cbf5(180) & Intra & 284 & 74 & 65 & 70 & 75 & $2.2 \mathrm{E}-21$ & 11.0 \\
\hline Cbf5(114)-Cbf5(161) & Intra & 268 & 74 & 66 & 68 & 60 & $2.3 \mathrm{E}-21$ & 24.0 \\
\hline Cbf5(137)-Nop10(18) & Inter & 234 & 61 & 64 & 47 & 62 & $2.0 \mathrm{E}-17$ & 19.0 \\
\hline Cbf5(114)-Cbf5(337) & Intra & 204 & 39 & 54 & 52 & 59 & $1.4 \mathrm{E}-17$ & 12.2 \\
\hline Cbf5(87)-Cbf5(114) & Intra & 181 & 42 & 44 & 48 & 47 & $1.1 \mathrm{E}-15$ & 14.7 \\
\hline Cbf5(180)-Nop10(18) & Inter & 160 & 42 & 35 & 43 & 40 & $6.1 \mathrm{E}-15$ & 13.0 \\
\hline Gar1(77)-Gar1(115) & Intra & 153 & 51 & 42 & 38 & 22 & $7.8 \mathrm{E}-16$ & 11.0 \\
\hline Nop10(1)-Nop10(19) & Intra & 145 & 37 & 35 & 39 & 34 & $9.5 \mathrm{E}-18$ & 11.5 \\
\hline Cbf5(180)-Nop10(19) & Inter & 98 & 30 & 23 & 22 & 23 & $2.0 \mathrm{E}-18$ & 15.5 \\
\hline Cbf5(80)-Cbf5(114) & Intra & 95 & 31 & 23 & 25 & 16 & $2.4 \mathrm{E}-26$ & 19.0 \\
\hline Cbf5(137)-Cbf5(180) & Intra & 84 & 15 & 20 & 24 & 25 & $5.0 \mathrm{E}-27$ & 13.6 \\
\hline Cbf5(161)-Gar1(115) & Inter & 68 & 12 & 16 & 26 & 14 & $1.5 \mathrm{E}-14$ & 18.9 \\
\hline Cbf5(9)-Cbf5(50) & Intra & 50 & 13 & 15 & 11 & 11 & $9.6 \mathrm{E}-12$ & 16.6 \\
\hline Nop10(28)-Nop10(40) & Intra & 44 & 6 & 12 & 13 & 13 & 7.1E-10 & 24.4 \\
\hline Cbf5(114)-Nop10(40) & Inter & 31 & 9 & 8 & 6 & 8 & 3.2E-11 & 35.0 \\
\hline Cbf5(134)-Gar1(104) & Inter & 29 & 6 & 9 & 6 & 8 & $2.3 \mathrm{E}-15$ & 18.4 \\
\hline Cbf5(31)-Cbf5(267) & Intra & 27 & 5 & 6 & 8 & 8 & $1.6 \mathrm{E}-17$ & 11.4 \\
\hline Cbf5(66)-Cbf5(114) & Intra & 27 & 2 & 8 & 9 & 8 & $1.7 \mathrm{E}-11$ & 19.4 \\
\hline Cbf5(161)-Gar1(77) & Inter & 19 & 5 & 5 & 4 & 5 & $1.8 \mathrm{E}-06$ & 18.9 \\
\hline Cbf5(50)-Nop10(40) & Inter & 17 & 1 & 8 & 4 & 4 & $4.9 \mathrm{E}-09$ & 31.8 \\
\hline Cbf5(180)-Nop10(40) & Inter & 16 & 4 & 5 & 4 & 3 & $1.8 \mathrm{E}-10$ & 41.8 \\
\hline Gar1(59)-Nop10(40) & Inter & 14 & 4 & 2 & 4 & 4 & $1.7 \mathrm{E}-06$ & 59.6 \\
\hline Cbf5(80)-Nop10(40) & Inter & 12 & - & 3 & 4 & 5 & $2.6 \mathrm{E}-10$ & 18.3 \\
\hline Nop10(18)-Nop10(28) & Intra & 12 & 3 & 3 & 2 & 4 & $9.2 \mathrm{E}-08$ & 9.7 \\
\hline Gar1(59)-Gar1(115) & Intra & 12 & - & 4 & 5 & 3 & $1.1 \mathrm{E}-07$ & 9.4 \\
\hline Nop10(19)-Nop10(40) & Intra & 10 & 2 & 4 & 1 & 3 & 2.7E-10 & 30.6 \\
\hline CDfS(114)-Nop10(28) & Inter & 8 & 4 & 1 & 3 & - & $1.8 \mathrm{E}-13$ & 33.1 \\
\hline СDf5(SO)-Cbł5(114) & Intra & 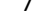 & - & 3 & 1 & 3 & $1.3 \mathrm{E}-12$ & 26.4 \\
\hline Cbł5(180)-Nop10(1) & Inter & 6 & - & 5 & 1 & - & $3.2 \mathrm{E}-15$ & 17.0 \\
\hline Cbf5(134)-Nop10(40) & Inter & 6 & 2 & 4 & - & - & $9.3 \mathrm{E}-09$ & 51.1 \\
\hline Cbf5(31)-Gar1(115) & Inter & 6 & 3 & 1 & 2 & - & $3.2 \mathrm{E}-06$ & 58.0 \\
\hline Cbf5(134)-Nop10(18) & Inter & 5 & - & 3 & 2 & - & $5.1 \mathrm{E}-05$ & 21.9 \\
\hline
\end{tabular}




\begin{tabular}{|c|c|c|c|c|c|c|c|c|}
\hline Cbf5(31)-Cbf5(50) & Intra & 4 & - & - & 2 & 2 & $4.4 \mathrm{E}-10$ & 23.5 \\
\hline Nhp2(32)-Nop10(28) & Inter & 178 & 44 & 42 & 45 & 47 & $1.0 \mathrm{E}-17$ & na \\
\hline Nhp2(61)-Nop10(40) & Inter & 129 & 27 & 34 & 32 & 36 & $3.8 \mathrm{E}-11$ & na \\
\hline Nhp2(65)-Nop10(40) & Inter & 87 & 21 & 18 & 23 & 25 & $3.8 \mathrm{E}-16$ & na \\
\hline Nhp2(69)-Nop10(40) & Inter & 84 & 19 & 19 & 21 & 25 & $7.3 \mathrm{E}-07$ & na \\
\hline Nop10(28)-Nop10(49) & Intra & 66 & 17 & 19 & 16 & 14 & $1.7 \mathrm{E}-07$ & na \\
\hline Nhp2(131)-Nhp2(133) & Intra & 63 & 18 & 15 & 14 & 16 & $7.0 \mathrm{E}-06$ & $\sim 5.9$ \\
\hline Cbf5(50)-Cbf5(370) & Intra & 48 & 10 & 10 & 13 & 15 & $1.0 \mathrm{E}-08$ & $\sim 27.8[\mathrm{Cbf} 5(50)-\mathrm{Cbf} 5(350)]$ \\
\hline Nhp2(65)-Nhp2(69) & Intra & 45 & 9 & 8 & 14 & 14 & $8.0 \mathrm{E}-06$ & $\sim 5.8$ \\
\hline Nhp2(69)-Nhp2(131) & Intra & 42 & 10 & 14 & 6 & 12 & $1.0 \mathrm{E}-05$ & $\sim 11.8$ \\
\hline Nhp2(45)-Nhp2(49) & Intra & 40 & 9 & 11 & 9 & 11 & $2.9 \mathrm{E}-06$ & $\sim 6.1$ \\
\hline Nhp2(61)-Nhp2(69) & Intra & 38 & 10 & 7 & 11 & 10 & $1.9 \mathrm{E}-05$ & $\sim 13.6$ \\
\hline Cbf5(114)-Cbf5(114) & Inter & 33 & 14 & 15 & 4 & - & $5.4 \mathrm{E}-08$ & na \\
\hline Nop10(40)-Nop10(49) & Intra & 31 & 9 & 8 & 7 & 7 & $2.4 \mathrm{E}-06$ & 32.6[Nop10(40)-Nop10(48)] \\
\hline Cbf5(363)-Cbf5(370) & Intra & 27 & 10 & 9 & 4 & 4 & $1.7 \mathrm{E}-06$ & na \\
\hline Cbf5(370)-Cbf5(383) & Intra & 26 & 5 & 7 & 6 & 8 & $7.6 \mathrm{E}-13$ & na \\
\hline Cbf5(114)-Cbf5(383) & Intra & 26 & 5 & 6 & 8 & 7 & $3.3 \mathrm{E}-09$ & 33.3[Cbf5(114)-Cbf5(350)] \\
\hline Cbf5(114)-Cbf5(370) & Intra & 22 & 3 & 4 & 8 & 7 & $8.8 \mathrm{E}-07$ & 33.3[Cbf5(114)-Cbf5(350)] \\
\hline Nhp2(54)-Nhp2(138) & Intra & 19 & 7 & 6 & 2 & 4 & $5.0 \mathrm{E}-06$ & $\sim 15.6$ \\
\hline Cbf5(180)-Nhp2(6) & Inter & 15 & 5 & 5 & 4 & 1 & $2.4 \mathrm{E}-12$ & na \\
\hline Cbf5(180)-Nhp2(12) & Inter & 15 & 4 & 5 & 4 & 2 & $1.8 \mathrm{E}-07$ & na \\
\hline Nhp2(6)-Nhp2(12) & Intra & 15 & 4 & 4 & 4 & 3 & 5.7E-07 & na \\
\hline Nhp2(54)-Nhp2(69) & Intra & 12 & 3 & 2 & 4 & 3 & 5.7E-05 & $\sim 17.6$ \\
\hline Nhp2(15)-Nop10(19) & Inter & 10 & 4 & 2 & 4 & - & $1.5 \mathrm{E}-14$ & na \\
\hline Cbf5(50)-Cbf5(383) & Intra & 10 & - & - & 5 & 5 & $2.0 \mathrm{E}-08$ & $\sim 27.8[\mathrm{Cbf5}(50)-\mathrm{Cbf5}(350)]$ \\
\hline Nhp2(133)-Nhp2(143) & Intra & 10 & 1 & 3 & 2 & 4 & 7.1E-05 & $\sim 16.4$ \\
\hline Nhp2(32)-Nhp2(38) & Intra & 9 & - & 3 & - & 6 & $1.2 \mathrm{E}-06$ & $\sim 16.3$ \\
\hline Cbf5(358)-Cbf5(370) & Intra & 7 & - & - & 4 & 3 & $1.9 \mathrm{E}-06$ & na \\
\hline Nhp2(57)-Nhp2(65) & Intra & 7 & - & 3 & 2 & 2 & $1.8 \mathrm{E}-05$ & $\sim 10.4$ \\
\hline Nhp2(117)-Nhp2(131) & Intra & 4 & 2 & 1 & 1 & - & $1.9 \mathrm{E}-08$ & $\sim 33.4$ \\
\hline Nhp2(136)-Nhp2(143) & Intra & 4 & - & - & 3 & 1 & $3.5 \mathrm{E}-05$ & $\sim 8.2$ \\
\hline Cbf5(358)-Cbf5(367) & Intra & 4 & - & 2 & - & 2 & 4.3E-05 & na \\
\hline Cbf5(134)-Nhp2(133) & Inter & 4 & - & - & 2 & 2 & 7.4E-05 & na \\
\hline \multicolumn{9}{|c|}{ EDC } \\
\hline Site-pairs & Inter/Intra & $\begin{array}{c}\text { \#total } \\
\text { spec }\end{array}$ & \#Exp1 & \#Exp2 & \#Еxp3 & \#Exp4 & Best E_value & Ca-Ca distance \\
\hline Aldolase(311)-Aldolase(354) & Intra & 40 & 8 & 7 & 11 & 14 & $1.5 \mathrm{E}-09$ & 11.8 \\
\hline Aldolase(52)-Aldolase(316) & Intra & 22 & - & 6 & 6 & 10 & 7.3E-09 & 15.4 \\
\hline Aldolase(41)-Aldolase(53) & Intra & 17 & 2 & 6 & 2 & 7 & $1.2 \mathrm{E}-06$ & 15.9 \\
\hline Aldolase(53)-Aldolase(317) & Intra & 13 & - & 4 & 4 & 5 & $3.1 \mathrm{E}-10$ & 11.8 \\
\hline Aldolase(52)-Aldolase(317) & Intra & 11 & 1 & - & 2 & 8 & $1.1 \mathrm{E}-08$ & 15.1 \\
\hline
\end{tabular}




\begin{tabular}{|c|c|c|c|c|c|c|c|c|}
\hline Aldolase(88)-Aldolase(317) & Intra & 11 & - & 1 & 4 & 6 & $1.5 \mathrm{E}-08$ & 12.3 \\
\hline Aldolase(317)-Aldolase(325) & Intra & 11 & - & 4 & 2 & 5 & $1.3 \mathrm{E}-07$ & 13.8 \\
\hline Aldolase(14)-Aldolase(100) & Intra & 10 & - & 5 & 2 & 3 & $1.8 \mathrm{E}-09$ & 20.1 \\
\hline Aldolase(41)-Aldolase(49) & Intra & 8 & - & 3 & 1 & 4 & $3.2 \mathrm{E}-11$ & 10.1 \\
\hline Aldolase(49)-Aldolase(317) & Intra & 8 & - & 3 & 2 & 3 & $6.5 \mathrm{E}-11$ & 13.3 \\
\hline Aldolase(10)-Aldolase(98) & Intra & 8 & - & 1 & 3 & 4 & $2.1 \mathrm{E}-10$ & 22.1 \\
\hline Aldolase(49)-Aldolase(316) & Intra & 7 & - & 3 & 3 & 1 & $5.4 \mathrm{E}-10$ & 11.4 \\
\hline Aldolase(1)-Aldolase(53) & Inter & 7 & - & 3 & 1 & 3 & $2.7 \mathrm{E}-04$ & 45.3 \\
\hline Aldolase(1)-Aldolase(33) & Inter & 6 & - & 2 & - & 4 & $9.9 \mathrm{E}-07$ & 31.1 \\
\hline Aldolase(41)-Aldolase(52) & Intra & 5 & 3 & - & - & 2 & $2.3 \mathrm{E}-09$ & 14.3 \\
\hline Aldolase(52)-Aldolase(98) & Intra & 5 & - & - & 2 & 3 & $1.9 \mathrm{E}-08$ & 22.6 \\
\hline Aldolase(49)-Aldolase(100) & Intra & 5 & - & 3 & 1 & 1 & $1.3 \mathrm{E}-04$ & 29.1 \\
\hline Aldolase(14)-Aldolase(329) & Intra & 4 & - & 1 & - & 3 & 7.4E-15 & 32.0 \\
\hline Aldolase(67)-Aldolase(100) & Intra & 4 & - & 2 & - & 2 & $4.4 \mathrm{E}-07$ & 6.9 \\
\hline Aldolase(316)-Aldolase(326) & Intra & 4 & - & 2 & 1 & 1 & $1.2 \mathrm{E}-05$ & 14.8 \\
\hline Aldolase(53)-Aldolase(98) & Intra & 4 & - & 3 & 1 & - & $3.3 \mathrm{E}-04$ & 22.1 \\
\hline Aldolase(52)-Aldolase(100) & Intra & 4 & - & - & 1 & 3 & $6.3 \mathrm{E}-04$ & 24.0 \\
\hline bsa(1)-bsa(12) & Intra & 83 & 27 & 20 & 20 & 16 & $9.4 \mathrm{E}-17$ & $\sim 10.4[\mathrm{bsa}(3)-\mathrm{bsa}(12)]$ \\
\hline bsa(45)-bsa(76) & Intra & 67 & 22 & 15 & 15 & 15 & $8.6 \mathrm{E}-13$ & 11.9 \\
\hline bsa(494)-bsa(537) & Intra & 46 & 9 & 13 & 13 & 11 & $5.2 \mathrm{E}-10$ & 9.3 \\
\hline bsa(130)-bsa(132) & Intra & 35 & 8 & 8 & 6 & 13 & $1.6 \mathrm{E}-15$ & 5.5 \\
\hline bsa(524)-bsa(555) & Intra & 32 & 8 & 9 & 8 & 7 & $8.8 \mathrm{E}-21$ & 12.5 \\
\hline bsa(204)-bsa(464) & Intra & 30 & 8 & 7 & 7 & 8 & $2.4 \mathrm{E}-13$ & 10.9 \\
\hline bsa(93)-bsa(107) & Intra & 28 & 4 & 10 & 5 & 9 & 3.7E-05 & 20.3 \\
\hline bsa(388)-bsa(441) & Intra & 21 & 9 & 5 & 3 & 4 & $2.3 \mathrm{E}-06$ & 9.2 \\
\hline bsa(464)-bsa(471) & Intra & 20 & 8 & 5 & 3 & 4 & $8.5 \mathrm{E}-13$ & 11.9 \\
\hline bsa(395)-bsa(431) & Intra & 17 & 4 & 5 & 4 & 4 & $2.7 \mathrm{E}-10$ & 12.3 \\
\hline bsa(6)-bsa(12) & Intra & 17 & 6 & 4 & 4 & 3 & 3.7E-09 & 9.9 \\
\hline bsa(4)-bsa(229) & Intra & 16 & 6 & 5 & 1 & 4 & $1.5 \mathrm{E}-08$ & 30.2 \\
\hline bsa(322)-bsa(351) & Intra & 15 & 7 & 1 & 3 & 4 & $3.8 \mathrm{E}-18$ & 14.1 \\
\hline bsa(399)-bsa(431) & Intra & 14 & 7 & 4 & 2 & 1 & $2.0 \mathrm{E}-07$ & 6.5 \\
\hline bsa(129)-bsa(132) & Intra & 13 & 4 & 2 & 3 & 4 & $6.3 \mathrm{E}-12$ & 5.5 \\
\hline bsa(4)-bsa(13) & Intra & 13 & 5 & 2 & 3 & 3 & $3.3 \mathrm{E}-09$ & 14.0 \\
\hline bsa(239)-bsa(243) & Intra & 13 & 5 & 3 & 4 & 1 & $1.4 \mathrm{E}-07$ & 5.9 \\
\hline bsa(211)-bsa(323) & Intra & 12 & 5 & 4 & 1 & 2 & $5.0 \mathrm{E}-12$ & 10.6 \\
\hline bsa(125)-bsa(136) & Intra & 12 & 4 & 4 & 1 & 3 & $6.8 \mathrm{E}-09$ & 10.2 \\
\hline bsa(12)-bsa(57) & Intra & 11 & 3 & 4 & 1 & 3 & $5.1 \mathrm{E}-16$ & 11.9 \\
\hline bsa(12)-bsa(56) & Intra & 11 & 5 & 3 & 3 & - & $6.0 \mathrm{E}-16$ & 12.7 \\
\hline bsa(166)-bsa(180) & Intra & 11 & - & 3 & 2 & 6 & $1.6 \mathrm{E}-10$ & 10.9 \\
\hline bsa(322)-bsa(363) & Intra & 11 & 3 & 3 & 1 & 4 & $3.9 \mathrm{E}-10$ & 20.0 \\
\hline
\end{tabular}




\begin{tabular}{|c|c|c|c|c|c|c|c|c|}
\hline bsa(182)-bsa(431) & Intra & 10 & 3 & 3 & 1 & 3 & $3.2 \mathrm{E}-07$ & 15.2 \\
\hline bsa(116)-bsa(182) & Intra & 10 & 4 & 2 & 2 & 2 & 2.7E-04 & 11.0 \\
\hline bsa(224)-bsa(299) & Intra & 9 & 5 & 3 & - & 1 & $2.4 \mathrm{E}-10$ & 9.6 \\
\hline bsa(37)-bsa(76) & Intra & 9 & - & 3 & 4 & 2 & $1.5 \mathrm{E}-06$ & 16.3 \\
\hline bsa(524)-bsa(548) & Intra & 8 & - & 3 & 3 & 2 & $1.1 \mathrm{E}-16$ & 11.4 \\
\hline bsa(535)-bsa(540) & Intra & 8 & 3 & 1 & 4 & - & $2.2 \mathrm{E}-05$ & 11.8 \\
\hline bsa(4)-bsa(57) & Intra & 7 & 2 & - & 3 & 2 & $2.5 \mathrm{E}-11$ & 6.4 \\
\hline bsa(4)-bsa(56) & Intra & 7 & 5 & 1 & 1 & - & $9.0 \mathrm{E}-11$ & 10.2 \\
\hline bsa(431)-bsa(443) & Intra & 7 & 4 & 2 & 1 & - & $6.5 \mathrm{E}-08$ & 16.3 \\
\hline bsa(424)-bsa(431) & Intra & 7 & 3 & 3 & 1 & - & 2.7E-06 & 10.9 \\
\hline bsa(4)-bsa(540) & Intra & 7 & 5 & 2 & - & - & $6.4 \mathrm{E}-06$ & 67.2 \\
\hline bsa(395)-bsa(439) & Intra & 7 & 3 & 1 & 1 & 2 & 7.4E-06 & 12.4 \\
\hline bsa(471)-bsa(540) & Intra & 7 & 2 & 2 & 2 & 1 & $1.3 \mathrm{E}-05$ & 23.6 \\
\hline bsa(431)-bsa(449) & Intra & 7 & - & 4 & - & 3 & $1.5 \mathrm{E}-05$ & 13.0 \\
\hline bsa(443)-bsa(471) & Intra & 6 & 2 & 2 & 1 & 1 & 3.7E-10 & 30.4 \\
\hline bsa(17)-bsa(131) & Intra & 6 & 2 & - & 2 & 2 & $5.3 \mathrm{E}-07$ & 9.5 \\
\hline bsa(471)-bsa(478) & Intra & 6 & 1 & 1 & 1 & 3 & $6.2 \mathrm{E}-06$ & 10.3 \\
\hline bsa(125)-bsa(132) & Intra & 5 & 2 & 3 & - & - & $1.3 \mathrm{E}-10$ & 8.5 \\
\hline bsa(4)-bsa(441) & Intra & 5 & 3 & 1 & 1 & - & $9.4 \mathrm{E}-10$ & 55.6 \\
\hline bsa(273)-bsa(276) & Intra & 5 & 1 & 2 & - & 2 & 4.7E-09 & 7.7 \\
\hline bsa(1)-bsa(275) & Intra & 5 & 4 & 1 & - & - & $5.4 \mathrm{E}-07$ & 32.9[bsa(3)-bsa(275)] \\
\hline bsa(4)-bsa(236) & Intra & 5 & 2 & - & - & 3 & $2.0 \mathrm{E}-06$ & 22.1 \\
\hline bsa(470)-bsa(474) & Intra & 5 & - & 1 & 3 & 1 & 7.9E-06 & 5.9 \\
\hline bsa(464)-bsa(474) & Intra & 5 & 2 & 3 & - & - & $8.8 \mathrm{E}-06$ & 11.0 \\
\hline bsa(441)-bsa(537) & Intra & 5 & 2 & 2 & 1 & - & $8.6 \mathrm{E}-05$ & 34.8 \\
\hline bsa(322)-bsa(353) & Intra & 4 & 2 & - & 2 & - & $1.4 \mathrm{E}-10$ & 9.0 \\
\hline bsa(4)-bsa(63) & Intra & 4 & 2 & - & 1 & 1 & $2.0 \mathrm{E}-08$ & 6.4 \\
\hline bsa(124)-bsa(136) & Intra & 4 & - & 2 & 2 & - & $6.9 \mathrm{E}-08$ & 13.8 \\
\hline bsa(56)-bsa(221) & Intra & 4 & 2 & 2 & - & - & $1.4 \mathrm{E}-05$ & 43.7 \\
\hline bsa(236)-bsa(537) & Intra & 4 & - & - & 2 & 2 & $6.6 \mathrm{E}-04$ & 51.5 \\
\hline bsa(93)-bsa(108) & Intra & 4 & 3 & - & 1 & - & 2.0E-03 & 23.2 \\
\hline Cbf5(267)-Cbf5(273) & Intra & 55 & 15 & 12 & 13 & 15 & $4.6 \mathrm{E}-15$ & 19.1 \\
\hline Nop10(18)-Nop10(22) & Intra & 28 & 5 & 6 & 8 & 9 & $1.5 \mathrm{E}-13$ & 8.8 \\
\hline Cbf5(18)-Cbf5(50) & Intra & 22 & 4 & 6 & 5 & 7 & $7.5 \mathrm{E}-10$ & 21.0 \\
\hline Cbf5(107)-Cbf5(114) & Intra & 9 & 3 & - & 3 & 3 & $2.1 \mathrm{E}-17$ & 14.1 \\
\hline Gar1(104)-Gar1(114) & Intra & 5 & - & 3 & - & 2 & $6.7 \mathrm{E}-08$ & 27.0 \\
\hline Nhp2(45)-Nhp2(144) & Intra & 55 & 9 & 17 & 11 & 18 & $6.4 \mathrm{E}-08$ & $\sim 15.2$ \\
\hline Nhp2(18)-Nhp2(37) & Intra & 37 & 8 & 13 & 8 & 8 & $2.8 \mathrm{E}-14$ & 23.2[Nhp2(27)-Nhp2(37)] \\
\hline Nhp2(132)-Nhp2(139) & Intra & 27 & 5 & 7 & 7 & 8 & $1.5 \mathrm{E}-08$ & $\sim 13.0$ \\
\hline Cbf5(367)-Cbf5(376) & Intra & 25 & 4 & 6 & 9 & 6 & $3.3 \mathrm{E}-12$ & na \\
\hline
\end{tabular}




\begin{tabular}{|c|c|c|c|c|c|c|c|c|}
\hline Nhp2(42)-Nhp2(148) & Intra & 14 & 3 & 5 & 1 & 5 & $1.2 \mathrm{E}-03$ & $\sim 12.5$ \\
\hline Cbf5(360)-Cbf5(376) & Intra & 12 & 3 & 4 & 2 & 3 & 4.4E-05 & na \\
\hline Cbf5(342)-Cbf5(358) & Intra & 11 & 2 & 4 & 1 & 4 & 3.3E-05 & 17.6[Cbf5(342)-Cbf5(350)] \\
\hline Cbf5(370)-Cbf5(376) & Intra & 10 & 4 & 1 & 2 & 3 & 8.1E-10 & na \\
\hline Cbf5(363)-Cbf5(376) & Intra & 10 & 2 & 5 & 1 & 2 & $7.2 \mathrm{E}-09$ & na \\
\hline Nhp2(132)-Nhp2(140) & Intra & 9 & 5 & 1 & 3 & - & 4.1E-08 & $\sim 15.8$ \\
\hline Cbf5(114)-Cbf5(376) & Intra & 7 & 1 & 2 & 2 & 2 & $2.0 \mathrm{E}-10$ & 33.3[Cbf5(114)-Cbf5(350)] \\
\hline Cbf5(367)-Cbf5(380) & Intra & 6 & 3 & 2 & 1 & - & $9.0 \mathrm{E}-12$ & na \\
\hline Nhp2(18)-Nhp2(38) & Intra & 6 & - & 4 & 2 & - & $6.8 \mathrm{E}-07$ & 25.9[Nhp2(27)-Nhp2(38)] \\
\hline Nhp2(9)-Nhp2(13) & Intra & 5 & 3 & 2 & - & - & $1.8 \mathrm{E}-06$ & na \\
\hline Nhp2(133)-Nhp2(140) & Intra & 5 & 1 & 2 & - & 2 & $1.4 \mathrm{E}-05$ & $\sim 14.8$ \\
\hline Nhp2(143)-Nhp2(152) & Intra & 5 & 3 & - & 1 & 1 & $2.4 \mathrm{E}-04$ & $\sim 14.2$ \\
\hline Nhp2(45)-Nhp2(140) & Intra & 5 & 1 & 1 & 3 & - & $6.6 \mathrm{E}-04$ & $\sim 17.6$ \\
\hline Nhp2(132)-Nhp2(144) & Intra & 4 & - & 1 & 1 & 2 & $8.4 \mathrm{E}-07$ & $\sim 20.6$ \\
\hline Nhp2(9)-Nop10(22) & Inter & 4 & 3 & - & 1 & - & $5.8 \mathrm{E}-06$ & na \\
\hline Nhp2(21)-Nhp2(38) & Intra & 4 & 1 & 1 & 2 & - & $1.9 \mathrm{E}-05$ & 25.9[Nhp2(27)-Nhp2(38)] \\
\hline Cbf5(171)-Nhp2(54) & Inter & 4 & - & 1 & 1 & 2 & $3.1 \mathrm{E}-03$ & na \\
\hline \multicolumn{9}{|c|}{ TFCS } \\
\hline Site-pairs & Inter/Intra & $\begin{array}{c}\text { \#total } \\
\text { spec }\end{array}$ & \#Exp1 & \#Exp2 & \#Еxp3 & \#Exp4 & Best E_value & Ca-Ca distance \\
\hline Aldolase(66)-Aldolase(86) & Intra & 128 & 29 & 29 & 45 & 25 & $1.9 \mathrm{E}-06$ & 17.3 \\
\hline Aldolase(229)-Aldolase(246) & Intra & 62 & 16 & 16 & 19 & 11 & $1.5 \mathrm{E}-13$ & 20.3 \\
\hline Aldolase(224)-Aldolase(242) & Inter & 40 & 5 & 14 & 11 & 10 & $2.7 \mathrm{E}-12$ & 26.8 \\
\hline Aldolase(318)-Aldolase(329) & Intra & 24 & 6 & 4 & 2 & 12 & $3.0 \mathrm{E}-06$ & 18.9 \\
\hline Aldolase(12)-Aldolase(14) & Intra & 17 & 9 & 5 & 3 & - & $1.5 \mathrm{E}-17$ & 5.4 \\
\hline Aldolase(89)-Aldolase(98) & Intra & 16 & 3 & 3 & 3 & 7 & $1.8 \mathrm{E}-04$ & 15.2 \\
\hline Aldolase(67)-Aldolase(86) & Intra & 12 & 4 & 4 & 2 & 2 & $4.9 \mathrm{E}-07$ & 18.7 \\
\hline Aldolase(311)-Aldolase(318) & Intra & 9 & 4 & 3 & 2 & - & 6.7E-07 & 13.0 \\
\hline Aldolase(316)-Aldolase(318) & Intra & 8 & 2 & 2 & 2 & 2 & $2.1 \mathrm{E}-04$ & 5.3 \\
\hline bsa(284)-bsa(294) & Intra & 45 & 3 & 15 & 16 & 11 & $8.4 \mathrm{E}-08$ & 18.1 \\
\hline bsa(556)-bsa(564) & Intra & 36 & 11 & 8 & 8 & 9 & $3.1 \mathrm{E}-14$ & 12.0 \\
\hline bsa(106)-bsa(118) & Inter & 31 & - & 9 & 12 & 10 & $2.0 \mathrm{E}-07$ & 25.8 \\
\hline bsa(279)-bsa(285) & Intra & 28 & 4 & 11 & 8 & 5 & 5.1E-09 & 7.2 \\
\hline bsa(375)-bsa(381) & Intra & 24 & 8 & 5 & 5 & 6 & $7.8 \mathrm{E}-10$ & 10.9 \\
\hline bsa(294)-bsa(313) & Intra & 24 & 3 & 8 & 4 & 9 & 1.7E-06 & 32.7 \\
\hline bsa(254)-bsa(261) & Intra & 20 & 2 & 6 & 8 & 4 & $9.8 \mathrm{E}-18$ & 10.8 \\
\hline bsa(93)-bsa(107) & Intra & 19 & - & 3 & 8 & 8 & $5.8 \mathrm{E}-05$ & 20.3 \\
\hline bsa(242)-bsa(258) & Intra & 17 & 4 & 7 & 4 & 2 & $4.8 \mathrm{E}-05$ & 15.3 \\
\hline bsa(293)-bsa(312) & Intra & 15 & 2 & 5 & 6 & 2 & $1.2 \mathrm{E}-06$ & 37.1 \\
\hline bsa(45)-bsa(64) & Intra & 13 & 4 & 5 & 1 & 3 & $2.6 \mathrm{E}-13$ & 15.8 \\
\hline bsa(544)-bsa(564) & Intra & 8 & 1 & 2 & 1 & 4 & $6.3 \mathrm{E}-09$ & 25.0 \\
\hline
\end{tabular}




\begin{tabular}{|c|c|c|c|c|c|c|c|c|}
\hline bsa(544)-bsa(561) & Inter & 8 & 3 & 3 & 2 & - & 4.4E-08 & 16.8 \\
\hline bsa(114)-bsa(118) & Intra & 8 & - & 2 & 2 & 4 & 3.6E-07 & 12.6 \\
\hline bsa(111)-bsa(116) & Intra & 7 & - & 2 & 4 & 1 & 9.7E-05 & 14.8 \\
\hline bsa(93)-bsa(100) & Intra & 6 & 2 & 2 & - & 2 & $1.1 \mathrm{E}-07$ & 9.9 \\
\hline bsa(107)-bsa(116) & Intra & 6 & - & - & 3 & 3 & 6.7E-06 & 21.6 \\
\hline bsa(152)-bsa(173) & Intra & 5 & 2 & 3 & - & - & $1.4 \mathrm{E}-06$ & 26.4 \\
\hline bsa(255)-bsa(261) & Intra & 4 & - & 1 & - & 3 & 2.1E-17 & 10.0 \\
\hline
\end{tabular}


Supplemental Table 2. Cross-links identified in the $70 \mathrm{~S}$ ribosome.

\begin{tabular}{|c|c|c|c|c|c|c|c|c|c|}
\hline \multicolumn{10}{|c|}{ GMBS } \\
\hline Site-pairs & \#total spec & \#Exp1 & \#Exp2 & \#Еxp3 & \#Exp4 & \#Exp5 & \#Exp6 & Best E_value & $\begin{array}{c}\mathrm{Ca}-\mathrm{Ca} \\
\text { distance }\end{array}$ \\
\hline \multicolumn{10}{|l|}{ Inter Molecular } \\
\hline RL32(57)-RL17(100) & 26 & 3 & 3 & 5 & 4 & 5 & 6 & 5.9E-08 & 12.0 \\
\hline RS3(16)-RS21(23) & 5 & & 4 & & & & 1 & 9.7E-07 & 45.7 \\
\hline \multicolumn{10}{|l|}{ Intra Molecular } \\
\hline RL17(35)-RL17(100) & 19 & & 4 & 4 & 6 & 2 & 3 & $5.0 \mathrm{E}-06$ & 8.9 \\
\hline \multicolumn{10}{|c|}{ Sulfo-GMBS } \\
\hline Site-pairs & \#total spec & \#Exp1 & \#Еxp2 & \#Exp3 & \#Exp4 & \#Exp5 & \#Exp6 & Best E_value & $\begin{array}{c}\mathrm{Ca}-\mathrm{Ca} \\
\text { distance }\end{array}$ \\
\hline \multicolumn{10}{|l|}{ Inter Molecular } \\
\hline RS3(16)-RS21(23) & 8 & & & 3 & 3 & & 2 & $9.9 \mathrm{E}-07$ & 45.7 \\
\hline RS11(70)-RL7(71) & 5 & 3 & 2 & & & & & 1.7E-06 & - \\
\hline RL32(57)-RL17(100) & 5 & & & 2 & 1 & 1 & 1 & $3.3 \mathrm{E}-07$ & 12.0 \\
\hline RS11(70)-RS7(149) & 4 & 1 & 3 & & & & & $3.2 \mathrm{E}-10$ & - \\
\hline RL21(1)-RL31(40) & 3 & 1 & 2 & & & & & $1.5 \mathrm{E}-06$ & - \\
\hline RS11(70)-RS7(131) & 2 & 1 & 1 & & & & & $8.8 \mathrm{E}-06$ & 31.4 \\
\hline \multicolumn{10}{|l|}{ Intra Molecular } \\
\hline RL17(35)-RL17(100) & 9 & 1 & 2 & 4 & 1 & & 1 & $3.8 \mathrm{E}-05$ & 8.9 \\
\hline RL2(6)-RL2(18) & 6 & 3 & 3 & & & & & $6.5 \mathrm{E}-08$ & 7.7 \\
\hline \multicolumn{10}{|c|}{$\mathrm{BS}^{2} \mathrm{G}$} \\
\hline Site-pairs & \#total spec & \#Exp1 & \#Exp2 & \#Еxp3 & \#Exp4 & \#Exp5 & \#Exp6 & Best E_value & $\begin{array}{l}\text { CanaCa } \\
\text { distance }\end{array}$ \\
\hline \multicolumn{10}{|l|}{ Inter Molecular } \\
\hline RS7(11)-RS9(100) & 80 & 14 & 14 & 12 & 12 & 13 & 15 & $2.4 \mathrm{E}-12$ & 14.3 \\
\hline RS8(89)-RS5(167) & 75 & 12 & 11 & 15 & 11 & 12 & 14 & 3.0E-20 & na \\
\hline RS8(22)-RS2(28) & 67 & 10 & 12 & 17 & 16 & 5 & 7 & $4.2 \mathrm{E}-16$ & 12.5 \\
\hline RL6(29)-RL7(71) & 63 & 11 & 11 & 8 & 12 & 12 & 9 & 3.0E-11 & na \\
\hline RL7(85)-RL6(86) & 63 & 10 & 12 & 10 & 9 & 9 & 13 & $1.3 \mathrm{E}-16$ & na \\
\hline RL7(71)-RL6(86) & 57 & 16 & 16 & 7 & 7 & 8 & 3 & 1.7E-11 & na \\
\hline RS3(108)-RS1(464) & 53 & 5 & 10 & 9 & 9 & 10 & 10 & $1.1 \mathrm{E}-10$ & na \\
\hline RS18(9)-RS21(25) & 52 & 16 & 15 & 3 & 3 & 10 & 5 & $2.2 \mathrm{E}-08$ & na \\
\hline RL6(29)-RL7(85) & 49 & 10 & 11 & 9 & 6 & 5 & 8 & $2.0 \mathrm{E}-13$ & na \\
\hline RS19(87)-RS13(118) & 37 & 4 & 4 & 7 & 5 & 8 & 9 & 1.7E-11 & na \\
\hline RL32(32)-RL17(121) & 31 & 14 & 7 & 1 & 3 & 4 & 2 & $1.4 \mathrm{E}-08$ & na \\
\hline RL19(2)-RL3(7) & 27 & 2 & 6 & 6 & 6 & 4 & 3 & $8.7 \mathrm{E}-12$ & 11.9 \\
\hline RS19(87)-RS13(114) & 23 & 5 & 3 & 4 & 3 & 5 & 3 & 4.5E-11 & na \\
\hline RS19(28)-RL31(70) & 23 & 4 & 3 & 3 & 5 & 3 & 5 & $1.2 \mathrm{E}-09$ & na \\
\hline RL33(33)-RL1(141) & 22 & 4 & 6 & 4 & 6 & 1 & 1 & $2.8 \mathrm{E}-07$ & na \\
\hline RS3(108)-RS1(450) & 21 & 3 & 4 & 7 & 7 & & & $5.0 \mathrm{E}-10$ & na \\
\hline RS19(17)-RL31(70) & 20 & 10 & 10 & & & & & $1.0 \mathrm{E}-09$ & na \\
\hline GyrB(14)-RL7(85) & 20 & 5 & 3 & 2 & 5 & 5 & & 3.5E-05 & na \\
\hline RS10(82)-RS1(450) & 18 & 6 & 6 & 3 & 3 & & & $5.1 \mathrm{E}-13$ & na \\
\hline RL1(14)-RL9(112) & 16 & 1 & 6 & 2 & 3 & 2 & 2 & $2.4 \mathrm{E}-08$ & na \\
\hline RL9(42)-RL28(44) & 15 & 9 & 6 & & & & & $3.4 \mathrm{E}-12$ & 21.2 \\
\hline
\end{tabular}




\begin{tabular}{|c|c|c|c|c|c|c|c|c|c|}
\hline RL7(71)-RL11(82) & 15 & 2 & 2 & 3 & 2 & 3 & 3 & $5.6 \mathrm{E}-08$ & na \\
\hline RS18(9)-RS6(93) & 14 & 2 & 4 & 5 & 3 & & & $7.9 \mathrm{E}-10$ & na \\
\hline RS18(30)-RS6(104) & 13 & 7 & 6 & & & & & $1.2 \mathrm{E}-14$ & na \\
\hline RL5(2)-RL31(39) & 13 & 9 & 4 & & & & & $1.5 \mathrm{E}-20$ & na \\
\hline RS2(1)-RS1(14) & 10 & 6 & 4 & & & & & $2.3 \mathrm{E}-07$ & na \\
\hline RL23(9)-RL29(54) & 10 & 2 & 3 & 2 & 2 & & 1 & $7.6 \mathrm{E}-06$ & 16.2 \\
\hline RS18(30)-RS6(106) & 9 & 3 & 6 & & & & & $1.2 \mathrm{E}-05$ & na \\
\hline RL31(70)-RL5(120) & 9 & 1 & & 1 & 2 & 3 & 2 & $1.6 \mathrm{E}-08$ & na \\
\hline RS18(9)-RS1(158) & 7 & & 1 & 1 & 3 & 2 & & $6.0 \mathrm{E}-06$ & na \\
\hline RS19(87)-RS13(117) & 7 & & & 2 & 1 & 3 & 1 & $4.6 \mathrm{E}-08$ & na \\
\hline RL13(12)-RL20(103) & 7 & 4 & 3 & & & & & $7.3 \mathrm{E}-09$ & 15.9 \\
\hline RL33(30)-RL1(141) & 7 & 3 & 2 & & 2 & & & $1.2 \mathrm{E}-04$ & na \\
\hline RS16(12)-RS21(54) & 7 & & & & & 4 & 3 & $2.1 \mathrm{E}-07$ & na \\
\hline RS1(162)-RS21(58) & 6 & & & 1 & & 2 & 3 & $9.3 \mathrm{E}-09$ & na \\
\hline RL5(2)-RL31(23) & 6 & 4 & 2 & & & & & $1.6 \mathrm{E}-11$ & na \\
\hline RL32(53)-RL17(121) & 6 & 3 & 1 & 2 & & & & $4.4 \mathrm{E}-06$ & na \\
\hline RS15(65)-RS7(136) & 5 & & & 3 & 1 & & 1 & $6.2 \mathrm{E}-07$ & 102.1 \\
\hline RL1(1)-RS12(108) & 5 & 3 & 2 & & & & & $8.5 \mathrm{E}-08$ & na \\
\hline CH60(226)-ATPA(384) & 5 & & & 1 & 3 & 1 & & $1.6 \mathrm{E}-11$ & na \\
\hline RL22(16)-RL22(98) & 4 & 1 & & 2 & 1 & & & $6.6 \mathrm{E}-08$ & 12.8 \\
\hline RS19(87)-RPOB(1028) & 4 & 2 & & 1 & 1 & & & $2.8 \mathrm{E}-05$ & na \\
\hline RL28(62)-RL1(198) & 4 & 2 & 2 & & & & & $4.2 \mathrm{E}-06$ & na \\
\hline RS10(30)-RL7(82) & 4 & 1 & 2 & & 1 & & & $3.1 \mathrm{E}-10$ & na \\
\hline RL1(54)-RS1(464) & 3 & 2 & & & & 1 & & $6.1 \mathrm{E}-10$ & na \\
\hline GyrB(14)-RL7(96) & 3 & 2 & & & 1 & & & $3.4 \mathrm{E}-04$ & na \\
\hline $\operatorname{HPF}(87)-R S 3(219)$ & 3 & & & 1 & & 1 & 1 & $3.6 \mathrm{E}-06$ & na \\
\hline RS8(50)-RPOC(345) & 3 & 1 & 2 & & & & & $1.5 \mathrm{E}-05$ & na \\
\hline YfiA(2)-RS19(87) & 3 & & & 1 & 2 & & & $2.0 \mathrm{E}-08$ & na \\
\hline RS2(66)-RL10(105) & 3 & & & & 1 & 1 & 1 & $8.5 \mathrm{E}-09$ & na \\
\hline RS2(128)-RS3(147) & 3 & 1 & 2 & & & & & $1.0 \mathrm{E}-05$ & 26.1 \\
\hline RL11(95)-DicA(113) & 2 & & 1 & & & 1 & & $5.7 \mathrm{E}-09$ & na \\
\hline RL1(3)-RS12(108) & 2 & 1 & 1 & & & & & $8.1 \mathrm{E}-08$ & na \\
\hline RS12(30)-RS3(108) & 2 & & & & 1 & & 1 & $4.1 \mathrm{E}-06$ & 77.9 \\
\hline RS18(50)-RS6(93) & 2 & & & 1 & 1 & & & $1.3 \mathrm{E}-05$ & na \\
\hline \multicolumn{10}{|l|}{ Intra Molecular } \\
\hline RL22(28)-RL22(70) & 132 & 52 & 53 & 5 & 5 & 10 & 7 & $2.2 \mathrm{E}-25$ & 3.9 \\
\hline RL1(54)-RL1(205) & 115 & 14 & 20 & 17 & 20 & 21 & 23 & $2.7 \mathrm{E}-18$ & na \\
\hline RS20(34)-RS20(49) & 108 & 18 & 21 & 18 & 18 & 16 & 17 & $2.9 \mathrm{E}-24$ & 9.6 \\
\hline RL7(71)-RL7(85) & 107 & 21 & 22 & 17 & 19 & 18 & 10 & $3.6 \mathrm{E}-10$ & na \\
\hline RL1(141)-RL1(198) & 95 & 19 & 17 & 18 & 17 & 15 & 9 & $4.2 \mathrm{E}-15$ & na \\
\hline RS3(108)-RS3(219) & 71 & 15 & 13 & 11 & 13 & 9 & 10 & $8.4 \mathrm{E}-15$ & na \\
\hline RL1(197)-RL1(200) & 69 & 16 & 17 & 8 & 10 & 9 & 9 & $2.3 \mathrm{E}-16$ & na \\
\hline RL6(6)-RL6(44) & 65 & 14 & 11 & 10 & 11 & 10 & 9 & $2.8 \mathrm{E}-21$ & 10.8 \\
\hline RS20(16)-RS20(19) & 61 & 7 & 9 & 11 & 14 & 13 & 7 & $5.1 \mathrm{E}-07$ & 5.9 \\
\hline RS9(2)-RS9(22) & 59 & 8 & 7 & 9 & 9 & 14 & 12 & $3.3 \mathrm{E}-11$ & na \\
\hline RS4(151)-RS4(156) & 57 & 12 & 18 & 7 & 9 & 4 & 7 & $8.0 \mathrm{E}-07$ & 5.1 \\
\hline RL5(47)-RL5(69) & 56 & 17 & 15 & 6 & 8 & 5 & 5 & $2.5 \mathrm{E}-17$ & 12.4 \\
\hline RS12(108)-RS12(120) & 55 & 9 & 16 & 3 & 6 & 11 & 10 & $1.8 \mathrm{E}-19$ & 9.6 \\
\hline
\end{tabular}




\begin{tabular}{|c|c|c|c|c|c|c|c|c|c|}
\hline RL10(37)-RL10(105) & 45 & 21 & 16 & 2 & & 5 & 1 & $5.6 \mathrm{E}-07$ & na \\
\hline RS12(108)-RS12(111) & 42 & 5 & 4 & 9 & 8 & 9 & 7 & $9.6 \mathrm{E}-10$ & 8.9 \\
\hline RS18(9)-RS18(30) & 41 & 9 & 11 & 3 & 6 & 7 & 5 & $1.7 \mathrm{E}-07$ & na \\
\hline RL1(54)-RL1(141) & 39 & 9 & 11 & 7 & 5 & 4 & 3 & $1.8 \mathrm{E}-12$ & na \\
\hline RL11(81)-RL11(87) & 38 & 10 & 11 & 5 & 4 & 4 & 4 & $3.0 \mathrm{E}-13$ & 4.8 \\
\hline RS3(86)-RS3(89) & 36 & 12 & 10 & 4 & 4 & 4 & 2 & $4.0 \mathrm{E}-07$ & 5.6 \\
\hline RL4(123)-RL4(137) & 36 & 14 & 15 & 3 & 2 & 1 & 1 & $1.1 \mathrm{E}-07$ & 12.7 \\
\hline RL1(141)-RL1(167) & 36 & 13 & 18 & 2 & 3 & & & $1.1 \mathrm{E}-13$ & na \\
\hline RS19(21)-RS19(29) & 35 & 3 & 4 & 12 & 9 & 5 & 2 & $9.4 \mathrm{E}-11$ & 13.2 \\
\hline RS2(2)-RS2(11) & 32 & 14 & 12 & 2 & 4 & & & $4.2 \mathrm{E}-09$ & na \\
\hline ATPA(384)-ATPA(388) & 32 & 6 & 6 & 5 & 5 & 5 & 5 & $4.3 \mathrm{E}-15$ & na \\
\hline RL2(183)-RL2(265) & 31 & 3 & 5 & 7 & 6 & 3 & 7 & $1.0 \mathrm{E}-09$ & 8.3 \\
\hline RS4(83)-RS4(185) & 31 & 11 & 8 & 4 & 2 & 4 & 2 & $2.4 \mathrm{E}-06$ & 14.8 \\
\hline RL16(118)-RL16(127) & 28 & 8 & 5 & 4 & 5 & 4 & 2 & $9.4 \mathrm{E}-07$ & 11.2 \\
\hline RL22(1)-RL22(73) & 27 & 4 & 11 & 4 & 3 & 2 & 3 & $3.8 \mathrm{E}-10$ & 12.7 \\
\hline RS1(279)-RS1(347) & 26 & 10 & 6 & 1 & 1 & 6 & 2 & $2.8 \mathrm{E}-10$ & na \\
\hline RL4(1)-RL4(137) & 25 & 7 & 7 & 3 & 3 & 2 & 3 & $1.2 \mathrm{E}-06$ & 28.4 \\
\hline RS2(28)-RS2(45) & 25 & 13 & 12 & & & & & $7.2 \mathrm{E}-12$ & 11.3 \\
\hline RL11(51)-RL11(82) & 21 & 7 & 7 & 3 & 4 & & & $2.7 \mathrm{E}-06$ & 12.0 \\
\hline RS10(1)-RS10(82) & 21 & 4 & 4 & 5 & 4 & & 4 & $3.1 \mathrm{E}-07$ & na \\
\hline RL24(26)-RL24(61) & 21 & 12 & 9 & & & & & $1.9 \mathrm{E}-12$ & 11.4 \\
\hline RL25(25)-RL25(46) & 19 & 6 & 7 & 2 & 3 & 1 & & $2.4 \mathrm{E}-10$ & 9.4 \\
\hline RS1(450)-RS1(504) & 19 & 13 & 6 & & & & & $4.2 \mathrm{E}-13$ & na \\
\hline RS11(2)-RS11(75) & 18 & 1 & 2 & 4 & 4 & 4 & 3 & $9.6 \mathrm{E}-07$ & na \\
\hline RL1(141)-RL1(200) & 17 & 5 & 8 & 4 & & & & $2.8 \mathrm{E}-08$ & na \\
\hline RL6(29)-RL6(134) & 17 & 9 & 8 & & & & & $2.1 \mathrm{E}-21$ & 14.0 \\
\hline RS3(49)-RS3(79) & 17 & 10 & 7 & & & & & $3.2 \mathrm{E}-10$ & 16.5 \\
\hline RL31(62)-RL31(70) & 16 & 6 & 2 & 2 & 1 & 2 & 3 & $2.2 \mathrm{E}-09$ & na \\
\hline RL1(54)-RL1(167) & 15 & 7 & 7 & & & & 1 & $4.3 \mathrm{E}-15$ & na \\
\hline RL25(10)-RL25(25) & 15 & 5 & 2 & 3 & 2 & 2 & 1 & $1.4 \mathrm{E}-05$ & 13.5 \\
\hline RL6(86)-RL6(134) & 14 & 4 & 10 & & & & & $2.7 \mathrm{E}-36$ & 8.4 \\
\hline RL24(91)-RL24(104) & 13 & & & 4 & 2 & 1 & 6 & $1.3 \mathrm{E}-08$ & na \\
\hline RL7(2)-RL7(71) & 13 & 1 & 2 & 3 & 2 & 2 & 3 & $4.1 \mathrm{E}-08$ & na \\
\hline RS19(18)-RS19(29) & 13 & 1 & 1 & 6 & 4 & 1 & & $3.3 \mathrm{E}-06$ & 11.8 \\
\hline RL7(66)-RL7(85) & 13 & 2 & & 1 & 4 & 4 & 2 & $4.2 \mathrm{E}-07$ & na \\
\hline RS1(150)-RS1(247) & 13 & 5 & 5 & & & 3 & & $1.7 \mathrm{E}-05$ & na \\
\hline RL7(71)-RL7(121) & 12 & 4 & 4 & 2 & 2 & & & $3.0 \mathrm{E}-07$ & na \\
\hline RL17(99)-RL17(42) & 10 & 1 & & 3 & 3 & 3 & & $2.0 \mathrm{E}-05$ & 10.3 \\
\hline RS3(108)-RS3(204) & 10 & 6 & 4 & & & & & $1.6 \mathrm{E}-08$ & na \\
\hline RL19(2)-RL19(6) & 9 & & & 4 & 3 & 2 & & $5.4 \mathrm{E}-06$ & na \\
\hline RL1(54)-RL1(200) & 9 & 2 & & 2 & 1 & 4 & & $1.3 \mathrm{E}-10$ & na \\
\hline RL33(33)-RL33(50) & 8 & 4 & 4 & & & & & $1.6 \mathrm{E}-07$ & 7.1 \\
\hline RL3(1)-RL3(204) & 8 & & 1 & 3 & 4 & & & $7.5 \mathrm{E}-09$ & 7.8 \\
\hline RL28(54)-RL28(62) & 7 & 2 & 3 & 2 & & & & $1.6 \mathrm{E}-06$ & 11.4 \\
\hline RL28(54)-RL28(61) & 6 & & 2 & & 1 & 3 & & $1.3 \mathrm{E}-05$ & 9.8 \\
\hline RL9(1)-RL9(22) & 6 & 1 & 1 & 4 & & & & $9.3 \mathrm{E}-22$ & 6.0 \\
\hline RL7(101)-RL7(109) & 5 & 1 & 2 & 1 & 1 & & & $2.8 \mathrm{E}-09$ & na \\
\hline RS9(2)-RS9(13) & 5 & & & 1 & 1 & 2 & 1 & $3.9 \mathrm{E}-06$ & na \\
\hline
\end{tabular}




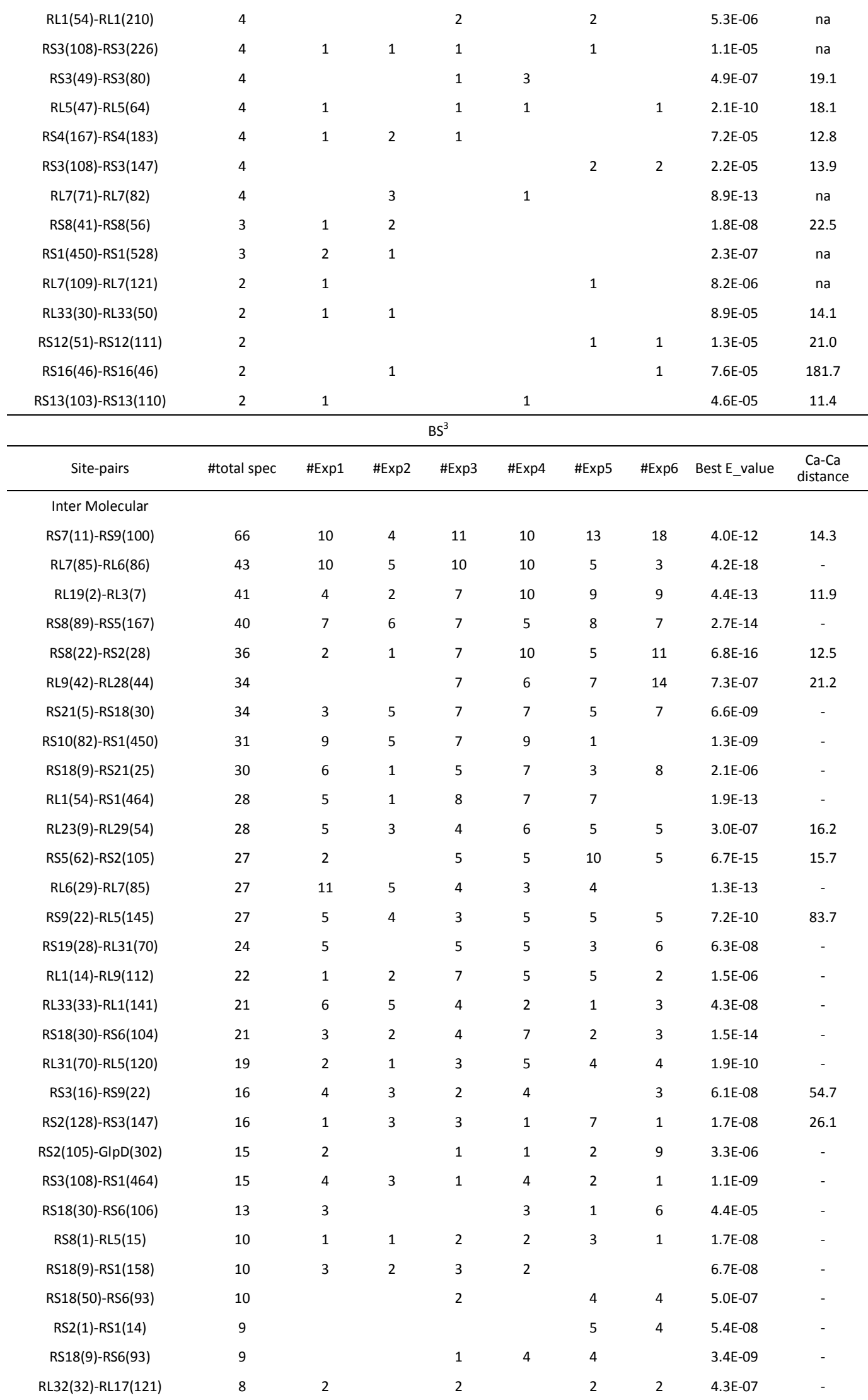




\begin{tabular}{|c|c|c|c|c|c|c|c|c|c|}
\hline RL24(43)-RS7(131) & 8 & & & & & 5 & 3 & $6.4 \mathrm{E}-10$ & 183.2 \\
\hline RS19(28)-RL5(120) & 7 & & & 3 & & 4 & & $4.4 \mathrm{E}-08$ & 24.3 \\
\hline RS14(7)-RS3(38) & 7 & & & 2 & 1 & 2 & 2 & $2.3 \mathrm{E}-07$ & 17.7 \\
\hline RL32(57)-RL17(121) & 7 & & & 2 & & 2 & 3 & $1.1 \mathrm{E}-05$ & - \\
\hline RS13(103)-RL5(120) & 7 & & & 3 & & 3 & 1 & $2.6 \mathrm{E}-04$ & 46.9 \\
\hline RL25(83)-RL16(127) & 5 & & & & & 4 & 1 & $6.3 \mathrm{E}-06$ & 12.6 \\
\hline RL32(53)-RL17(121) & 5 & 2 & & 1 & & & 2 & 4.9E-05 & - \\
\hline RL7(71)-RL6(86) & 5 & 3 & & & 2 & & & $1.5 \mathrm{E}-05$ & - \\
\hline RS3(16)-RL9(42) & 4 & 1 & & 2 & & 1 & & 7.6E-08 & 125.0 \\
\hline RS2(112)-RS3(147) & 4 & 1 & & 1 & 2 & & & $2.4 \mathrm{E}-04$ & 20.6 \\
\hline RL25(46)-RS7(131) & 4 & & & 2 & 2 & & & $2.5 \mathrm{E}-10$ & 135.4 \\
\hline RL7(71)-RL1(154) & 4 & 1 & & 1 & & 2 & & $1.2 \mathrm{E}-05$ & - \\
\hline RL31(70)-LPD(237) & 4 & & & 1 & 2 & 1 & & $2.3 \mathrm{E}-04$ & - \\
\hline RL4(123)-PROQ(160) & 3 & & & 1 & 1 & 1 & & 4.7E-05 & - \\
\hline RS16(46)-NUOB(204) & 3 & 1 & & & 1 & 1 & & $3.4 \mathrm{E}-05$ & - \\
\hline RS16(46)-RF2(334) & 3 & 1 & & & & 1 & 1 & $1.5 \mathrm{E}-04$ & - \\
\hline RL5(15)-RS22(16) & 3 & 1 & & & & 1 & 1 & $1.3 \mathrm{E}-04$ & - \\
\hline RS3(80)-TIG(287) & 3 & 1 & & & & 2 & & $2.1 \mathrm{E}-09$ & - \\
\hline RL22(73)-NUOCD(405) & 2 & 1 & & 1 & & & & $6.2 \mathrm{E}-05$ & - \\
\hline RL20(78)-TIG(272) & 2 & & & & 1 & 1 & & $5.0 \mathrm{E}-04$ & - \\
\hline RS5(14)-Gcd(514) & 2 & 1 & & & 1 & & & $7.3 \mathrm{E}-05$ & - \\
\hline RS15(1)-RL19(6) & 2 & & 1 & & 1 & & & $2.1 \mathrm{E}-05$ & - \\
\hline RL27(72)-RS12(108) & 2 & & & 1 & & & 1 & $1.7 \mathrm{E}-04$ & 120.2 \\
\hline RS14(47)-RL31(70) & 2 & & & & & 1 & 1 & $2.0 \mathrm{E}-08$ & - \\
\hline $\operatorname{SdhB}(1)-\operatorname{RS} 4(83)$ & 2 & & & 1 & & 1 & & $1.3 \mathrm{E}-04$ & - \\
\hline RL31(39)-RL5(145) & 2 & 1 & & 1 & & & & $4.0 \mathrm{E}-04$ & - \\
\hline Intra Molecular & & & & & & & & & \\
\hline RL1(141)-RL1(167) & 251 & 62 & 28 & 51 & 43 & 33 & 34 & $1.5 \mathrm{E}-21$ & - \\
\hline RL7(71)-RL7(85) & 218 & 62 & 31 & 43 & 32 & 28 & 22 & $1.2 \mathrm{E}-08$ & - \\
\hline RL22(1)-RL22(70) & 211 & 32 & 12 & 41 & 34 & 44 & 48 & $2.6 \mathrm{E}-17$ & 11.4 \\
\hline RL2(97)-RL2(125) & 152 & 8 & 1 & 29 & 29 & 38 & 47 & $8.6 \mathrm{E}-17$ & 18.2 \\
\hline RL18(63)-RL18(76) & 88 & 12 & 7 & 14 & 15 & 18 & 22 & $6.7 \mathrm{E}-14$ & 16.2 \\
\hline RL5(47)-RL5(64) & 88 & 13 & 7 & 18 & 15 & 20 & 15 & $1.8 \mathrm{E}-18$ & 18.1 \\
\hline RL6(6)-RL6(44) & 82 & 15 & 9 & 14 & 13 & 14 & 17 & $6.7 \mathrm{E}-20$ & 10.8 \\
\hline RS4(151)-RS4(156) & 79 & 10 & 4 & 13 & 12 & 21 & 19 & $7.0 \mathrm{E}-07$ & 5.1 \\
\hline RL20(78)-RL20(85) & 75 & 7 & 4 & 13 & 11 & 16 & 24 & $3.1 \mathrm{E}-15$ & 10.0 \\
\hline RS3(108)-RS3(147) & 73 & 15 & 6 & 12 & 16 & 11 & 13 & $2.2 \mathrm{E}-08$ & 13.9 \\
\hline RL1(141)-RL1(198) & 72 & 14 & 11 & 15 & 14 & 9 & 9 & $1.1 \mathrm{E}-18$ & - \\
\hline RS20(34)-RS20(49) & 69 & 10 & 5 & 12 & 13 & 12 & 17 & $1.7 \mathrm{E}-20$ & 9.6 \\
\hline RL5(47)-RL5(69) & 67 & 13 & 10 & 14 & 12 & 11 & 7 & $3.3 \mathrm{E}-23$ & 12.4 \\
\hline RL10(37)-RL10(105) & 59 & 10 & 3 & 12 & 6 & 11 & 17 & $1.0 \mathrm{E}-07$ & - \\
\hline RL4(1)-RL4(137) & 55 & 8 & 7 & 10 & 9 & 8 & 13 & $1.6 \mathrm{E}-08$ & 28.4 \\
\hline RL22(1)-RL22(73) & 55 & 3 & 4 & 11 & 10 & 15 & 12 & $1.0 \mathrm{E}-13$ & 12.7 \\
\hline RL2(183)-RL2(265) & 47 & 5 & 4 & 8 & 8 & 12 & 10 & $6.2 \mathrm{E}-09$ & 8.3 \\
\hline RL2(108)-RL2(125) & 46 & 7 & 3 & 10 & 9 & 9 & 8 & $3.9 \mathrm{E}-11$ & 10.7 \\
\hline RS9(2)-RS9(27) & 43 & 3 & 3 & 6 & 6 & 11 & 14 & $2.4 \mathrm{E}-16$ & - \\
\hline RS10(1)-RS10(82) & 42 & 8 & 2 & 9 & 8 & 9 & 6 & $5.2 \mathrm{E}-07$ & - \\
\hline RS12(108)-RS12(111) & 39 & 1 & & 9 & 10 & 9 & 10 & $6.3 \mathrm{E}-08$ & 8.9 \\
\hline
\end{tabular}




\begin{tabular}{|c|c|c|c|c|c|c|c|c|c|}
\hline RL25(25)-RL25(46) & 38 & 5 & & 9 & 4 & 9 & 11 & $9.6 \mathrm{E}-10$ & 9.4 \\
\hline RS9(2)-RS9(22) & 37 & 3 & 1 & 8 & 7 & 8 & 10 & $9.0 \mathrm{E}-09$ & - \\
\hline RL1(54)-RL1(205) & 36 & 4 & 3 & 2 & 3 & 11 & 13 & $1.0 \mathrm{E}-10$ & - \\
\hline RS10(30)-RS10(82) & 35 & 6 & 2 & 7 & 6 & 6 & 8 & $1.0 \mathrm{E}-26$ & 10.4 \\
\hline RL5(120)-RL5(179) & 31 & 5 & 1 & 7 & 7 & 8 & 3 & $2.2 \mathrm{E}-12$ & - \\
\hline RS3(86)-RS3(89) & 30 & 4 & 1 & 6 & 7 & 7 & 5 & $7.4 \mathrm{E}-06$ & 5.6 \\
\hline RL25(10)-RL25(25) & 30 & 5 & 4 & 6 & 4 & 6 & 5 & $8.2 \mathrm{E}-06$ & 13.5 \\
\hline RL11(81)-RL11(87) & 24 & 4 & 7 & 4 & 4 & 3 & 2 & $2.9 \mathrm{E}-14$ & 4.8 \\
\hline RL9(1)-RL9(22) & 23 & 4 & 3 & 4 & 4 & 4 & 4 & $2.0 \mathrm{E}-17$ & 6.0 \\
\hline ATPA(384)-ATPA(388) & 21 & 4 & 4 & 2 & 4 & 4 & 3 & $2.1 \mathrm{E}-12$ & - \\
\hline RL1(54)-RL1(141) & 19 & 10 & 4 & 3 & & 2 & & $1.0 \mathrm{E}-14$ & - \\
\hline RL22(28)-RL22(70) & 19 & 2 & & 3 & & 14 & & $3.5 \mathrm{E}-13$ & 3.9 \\
\hline RS4(83)-RS4(185) & 19 & & & 7 & 2 & 5 & 5 & $2.7 \mathrm{E}-06$ & 14.8 \\
\hline RS1(279)-RS1(347) & 18 & 2 & & 3 & 4 & 5 & 4 & $2.4 \mathrm{E}-11$ & - \\
\hline RL31(62)-RL31(70) & 18 & 3 & & 3 & 5 & 2 & 5 & $1.4 \mathrm{E}-10$ & - \\
\hline $\mathrm{RS} 2(28)-\mathrm{RS} 2(45)$ & 18 & & & & 4 & 2 & 12 & $2.8 \mathrm{E}-11$ & 11.3 \\
\hline RL20(85)-RL20(112) & 18 & 1 & & 2 & 1 & 6 & 8 & 3.3E-12 & 9.7 \\
\hline RL9(35)-RL9(112) & 17 & 3 & & 5 & 2 & 4 & 3 & $1.1 \mathrm{E}-18$ & 42.5 \\
\hline RL4(6)-RL4(137) & 17 & 3 & 2 & 4 & 3 & 3 & 2 & $7.2 \mathrm{E}-06$ & 14.5 \\
\hline RS3(49)-RS3(79) & 17 & 8 & 2 & 3 & 4 & & & $6.2 \mathrm{E}-12$ & 16.5 \\
\hline RS2(64)-RS2(115) & 16 & 2 & 1 & 4 & 4 & 4 & 1 & $2.1 \mathrm{E}-16$ & 24.1 \\
\hline RS2(2)-RS2(59) & 14 & 4 & 1 & 4 & 4 & 1 & & $3.8 \mathrm{E}-11$ & - \\
\hline RS3(80)-RS3(89) & 14 & 5 & 5 & 2 & 2 & & & $3.6 \mathrm{E}-10$ & 15.6 \\
\hline RL7(71)-RL7(82) & 14 & 9 & 5 & & & & & $9.6 \mathrm{E}-15$ & - \\
\hline RS1(247)-RS1(260) & 13 & 2 & & 3 & 5 & 1 & 2 & $1.9 \mathrm{E}-05$ & - \\
\hline RS3(45)-RS3(89) & 13 & 2 & & 3 & 4 & 4 & & $8.6 \mathrm{E}-08$ & 15.8 \\
\hline RL16(118)-RL16(127) & 13 & 3 & 1 & 1 & 4 & 2 & 2 & $6.1 E-06$ & 11.2 \\
\hline RS4(167)-RS4(183) & 12 & 1 & & 3 & 2 & 1 & 5 & $9.9 \mathrm{E}-07$ & 12.8 \\
\hline RL9(8)-RL9(35) & 11 & & & & 3 & 5 & 3 & $3.8 \mathrm{E}-05$ & 8.0 \\
\hline RL1(141)-RL1(200) & 11 & 4 & 3 & 2 & 2 & & & $6.0 \mathrm{E}-09$ & - \\
\hline RL7(66)-RL7(85) & 10 & 7 & 3 & & & & & $3.2 \mathrm{E}-06$ & - \\
\hline RS18(9)-RS18(30) & 9 & 3 & & 3 & & 3 & & $1.9 \mathrm{E}-06$ & - \\
\hline RS3(108)-RS3(219) & 9 & 3 & 2 & 2 & 2 & & & 4.4E-06 & - \\
\hline RS7(114)-RS7(131) & 9 & & & & 2 & 5 & 2 & $6.2 \mathrm{E}-08$ & 18.9 \\
\hline RL1(197)-RL1(200) & 9 & 1 & & 4 & 3 & & 1 & $1.8 \mathrm{E}-14$ & - \\
\hline RL1(54)-RL1(167) & 8 & & & & 2 & 1 & 5 & $1.2 \mathrm{E}-12$ & - \\
\hline RS2(66)-RS2(115) & 8 & 3 & & 1 & & 2 & 2 & $2.6 \mathrm{E}-19$ & 20.1 \\
\hline RL19(87)-RL19(106) & 8 & & & 2 & 3 & 3 & & $5.9 \mathrm{E}-05$ & 19.6 \\
\hline RS16(46)-RS16(80) & 8 & 1 & & 2 & 1 & 3 & 1 & $3.8 \mathrm{E}-05$ & 19.3 \\
\hline RS20(16)-RS20(19) & 6 & & & & & 2 & 4 & $7.2 \mathrm{E}-09$ & 5.9 \\
\hline RS12(108)-RS12(120) & 6 & & & 2 & & 2 & 2 & $9.0 \mathrm{E}-13$ & 9.6 \\
\hline RL16(34)-RL16(127) & 5 & 4 & 1 & & & & & $5.2 \mathrm{E}-14$ & 9.0 \\
\hline YfiA(2)-YfiA(29) & 5 & 1 & & 1 & & 3 & & $1.4 \mathrm{E}-08$ & - \\
\hline RS9(2)-RS9(13) & 5 & & 1 & 2 & 2 & & & $4.0 \mathrm{E}-06$ & - \\
\hline RS13(78)-RS13(103) & 5 & & & 1 & & 2 & 2 & $1.9 \mathrm{E}-07$ & 26.3 \\
\hline RS1(150)-RS1(247) & 5 & 2 & 2 & 1 & & & & $3.3 E-06$ & - \\
\hline RS19(28)-RS19(29) & 4 & & 2 & & & 1 & 1 & $1.3 \mathrm{E}-07$ & 3.8 \\
\hline RS2(112)-RS2(115) & 4 & & & 1 & 1 & 1 & 1 & $1.6 \mathrm{E}-05$ & 5.0 \\
\hline
\end{tabular}




\begin{tabular}{|c|c|c|c|c|c|c|c|c|c|}
\hline $\mathrm{CH} 60$ (34)-CH60(117) & 3 & & & 1 & 1 & 1 & & $2.4 \mathrm{E}-11$ & - \\
\hline RS2(2)-RS2(11) & 3 & & & & 1 & & 2 & $9.8 \mathrm{E}-09$ & - \\
\hline RL7(109)-RL7(121) & 2 & & & & 1 & 1 & & $7.5 \mathrm{E}-05$ & - \\
\hline RS4(77)-RS4(83) & 2 & & & & 1 & 1 & & $1.3 \mathrm{E}-04$ & 11.8 \\
\hline RL1(54)-RL1(210) & 2 & & & 1 & 1 & & & $1.6 \mathrm{E}-05$ & - \\
\hline $\operatorname{RL7}(2)-\operatorname{RL} 7(71)$ & 2 & 1 & 1 & & & & & $8.9 \mathrm{E}-06$ & - \\
\hline RS4(156)-RS4(177) & 2 & & & & 1 & & 1 & $1.3 \mathrm{E}-12$ & 11.3 \\
\hline RS8(41)-RS8(56) & 2 & & & & & 1 & 1 & $1.2 \mathrm{E}-06$ & 22.5 \\
\hline RS11(2)-RS11(75) & 2 & & & 1 & & & 1 & $3.2 \mathrm{E}-04$ & - \\
\hline \multicolumn{10}{|c|}{ DSS } \\
\hline Site-pairs & \#total spec & \#Exp1 & \#Exp2 & \#Ехp3 & \#Exp4 & \#Exp5 & \#Exp6 & Best E_value & $\begin{array}{c}\mathrm{Ca}-\mathrm{Ca} \\
\text { distance }\end{array}$ \\
\hline \multicolumn{10}{|l|}{ Inter Molecular } \\
\hline RS18(9)-RS21(25) & 89 & 11 & 15 & 17 & 17 & 15 & 14 & $1.4 \mathrm{E}-08$ & - \\
\hline RS18(30)-RS6(104) & 77 & 11 & 11 & 19 & 10 & 12 & 14 & $1.5 \mathrm{E}-22$ & - \\
\hline RS18(30)-RS6(106) & 72 & 12 & 11 & 13 & 12 & 12 & 12 & $1.1 \mathrm{E}-05$ & - \\
\hline RL9(42)-RL28(44) & 69 & 16 & 14 & 9 & 14 & 8 & 8 & $2.1 \mathrm{E}-14$ & 21.2 \\
\hline RL7(85)-RL6(86) & 53 & 9 & 9 & 6 & 10 & 9 & 10 & $5.1 \mathrm{E}-24$ & - \\
\hline RS21(5)-RS18(30) & 53 & 7 & 6 & 11 & 9 & 13 & 7 & $8.8 \mathrm{E}-10$ & - \\
\hline RL32(32)-RL17(121) & 51 & 8 & 8 & 8 & 7 & 9 & 11 & $1.0 \mathrm{E}-10$ & - \\
\hline RS2(128)-RS3(219) & 48 & 7 & 10 & 14 & 9 & 3 & 5 & $9.6 \mathrm{E}-15$ & - \\
\hline RS21(25)-RS18(38) & 46 & 9 & 7 & 8 & 4 & 10 & 8 & 1.7E-14 & 13.8 \\
\hline $\mathrm{RS7}(11)-\mathrm{RS} 9(100)$ & 41 & 9 & 7 & 4 & 5 & 8 & 8 & $9.3 \mathrm{E}-13$ & 14.3 \\
\hline RS18(9)-RS1(158) & 37 & 6 & 7 & 5 & 7 & 6 & 6 & $7.5 \mathrm{E}-08$ & - \\
\hline RS3(108)-RS1(464) & 33 & 7 & 8 & 5 & 9 & & 4 & $1.4 \mathrm{E}-10$ & - \\
\hline RL7(71)-RL11(82) & 33 & 7 & 8 & 3 & 6 & 6 & 3 & $3.3 \mathrm{E}-08$ & - \\
\hline RL23(9)-RL29(54) & 32 & 6 & 7 & 6 & 1 & 6 & 6 & $4.5 \mathrm{E}-06$ & 16.2 \\
\hline RS10(82)-RS3(225) & 32 & 5 & 6 & 8 & 6 & 4 & 3 & $2.0 \mathrm{E}-14$ & - \\
\hline RS10(30)-RS3(226) & 31 & 8 & 4 & 7 & 7 & 3 & 2 & $4.1 \mathrm{E}-14$ & - \\
\hline RS8(89)-RS5(167) & 31 & 6 & 8 & 1 & 3 & 6 & 7 & $1.3 \mathrm{E}-20$ & - \\
\hline RL7(71)-RL6(86) & 30 & 6 & 6 & 3 & 4 & 4 & 7 & 3.7E-10 & - \\
\hline RS2(128)-RS3(226) & 29 & 13 & 8 & 3 & & & 5 & $6.0 \mathrm{E}-10$ & - \\
\hline RS2(128)-RS3(225) & 28 & 5 & 7 & 8 & 7 & 1 & & $4.6 \mathrm{E}-19$ & - \\
\hline RL18(17)-RL27(62) & 26 & 8 & 6 & 1 & 6 & 3 & 2 & $1.8 \mathrm{E}-16$ & 15.2 \\
\hline RL6(29)-RL7(85) & 25 & 8 & 9 & & 8 & & & $3.2 \mathrm{E}-13$ & - \\
\hline RS2(128)-RS3(147) & 24 & 5 & 3 & 4 & 5 & 4 & 3 & $7.5 \mathrm{E}-10$ & 26.1 \\
\hline RS10(82)-RS3(226) & 24 & 4 & 7 & 2 & 6 & 2 & 3 & $1.3 \mathrm{E}-05$ & - \\
\hline RS19(28)-RL31(70) & 23 & 4 & 5 & 3 & 4 & 3 & 4 & $1.9 \mathrm{E}-09$ & - \\
\hline RL33(33)-RL1(141) & 22 & 6 & 5 & 3 & 4 & 1 & 3 & $2.9 \mathrm{E}-08$ & - \\
\hline RS10(30)-RS3(225) & 18 & 6 & 2 & 5 & 5 & & & $3.9 \mathrm{E}-13$ & - \\
\hline RL19(2)-RL3(7) & 17 & 2 & 3 & 2 & 4 & 3 & 3 & $6.6 \mathrm{E}-14$ & 11.9 \\
\hline RL28(44)-RL9(57) & 15 & & 3 & 5 & 4 & 3 & & $3.1 \mathrm{E}-09$ & 34.6 \\
\hline RL11(82)-RL7(85) & 14 & 3 & 5 & & 4 & 1 & 1 & $1.2 \mathrm{E}-05$ & - \\
\hline RS2(1)-RS1(14) & 14 & 3 & & 1 & 5 & 5 & & $3.8 \mathrm{E}-10$ & - \\
\hline $\mathrm{RS} 10(82)-\operatorname{RS} 1(450)$ & 13 & 5 & 4 & 1 & 3 & & & $1.7 \mathrm{E}-11$ & - \\
\hline RL9(89)-RL2(183) & 12 & 3 & 3 & & 3 & & 3 & $1.0 \mathrm{E}-10$ & 88.7 \\
\hline RL7(96)-RL10(105) & 12 & 4 & 4 & & 4 & & & $9.2 \mathrm{E}-08$ & - \\
\hline RL21(1)-RL32(7) & 11 & 3 & 1 & 1 & 3 & 3 & & $1.2 \mathrm{E}-05$ & 62.4 \\
\hline
\end{tabular}




\begin{tabular}{|c|c|c|c|c|c|c|c|c|c|}
\hline RL32(37)-RL22(48) & 9 & 3 & 3 & & & & 3 & $1.3 \mathrm{E}-08$ & 16.7 \\
\hline RL22(28)-RL32(37) & 9 & 3 & 4 & & & & 2 & $2.6 \mathrm{E}-06$ & 16.8 \\
\hline RS19(17)-RL31(70) & 8 & 1 & 1 & 5 & & & 1 & $1.4 \mathrm{E}-06$ & - \\
\hline RS21(25)-RS1(158) & 8 & 3 & 3 & & 2 & & & $2.5 \mathrm{E}-05$ & - \\
\hline RL1(54)-RS1(464) & 7 & 1 & 3 & & 3 & & & $1.9 \mathrm{E}-10$ & - \\
\hline RS10(30)-RS3(219) & 6 & & 1 & 1 & 4 & & & $2.3 \mathrm{E}-10$ & - \\
\hline SUCC(56)-RS1(363) & 6 & & 3 & 2 & 1 & & & $5.2 \mathrm{E}-06$ & - \\
\hline RS14(7)-RS3(38) & 5 & 1 & & & 2 & 1 & 1 & $5.3 \mathrm{E}-06$ & 17.7 \\
\hline RL27(5)-RL17(40) & 5 & 3 & 2 & & & & & $4.1 \mathrm{E}-05$ & - \\
\hline RS3(108)-RS1(450) & 4 & & 2 & 2 & & & & $9.3 \mathrm{E}-10$ & - \\
\hline RS6(106)-RS1(158) & 4 & 3 & & & & 1 & & $2.0 \mathrm{E}-06$ & - \\
\hline RL25(71)-RS1(363) & 4 & & 2 & & 2 & & & $1.5 \mathrm{E}-05$ & - \\
\hline RS9(2)-RS3(226) & 4 & & 2 & & & 1 & 1 & $5.8 \mathrm{E}-04$ & - \\
\hline RL32(53)-RL17(121) & 4 & 1 & & & 1 & 2 & & $8.8 \mathrm{E}-06$ & - \\
\hline RL19(106)-RL19(111) & 3 & & & & & 1 & 2 & $4.8 \mathrm{E}-04$ & 11.0 \\
\hline RS17(16)-RS15(71) & 3 & & & & & 1 & 2 & $3.8 \mathrm{E}-13$ & 24.6 \\
\hline RS9(27)-RL5(145) & 3 & & 1 & 2 & & & & $2.5 \mathrm{E}-07$ & 73.3 \\
\hline YfiA(2)-RS13(114) & 3 & & 1 & & 1 & & 1 & $1.6 \mathrm{E}-05$ & - \\
\hline RL7(85)-RL6(99) & 3 & 2 & 1 & & & & & $2.2 \mathrm{E}-14$ & - \\
\hline RL32(57)-RL17(121) & 3 & & 2 & & & 1 & & $1.7 \mathrm{E}-04$ & - \\
\hline RL5(69)-RhlE(418) & 3 & 1 & 1 & & 1 & & & $2.1 \mathrm{E}-05$ & - \\
\hline RL19(6)-RL3(7) & 2 & 1 & & & 1 & & & $2.3 \mathrm{E}-04$ & 16.5 \\
\hline RL22(1)-RL2(71) & 2 & & 1 & & & & 1 & 4.7E-05 & 129.6 \\
\hline $\mathrm{CH} 10(13)-\operatorname{Sec} Y(20)$ & 2 & 1 & & & & 1 & & $6.4 \mathrm{E}-06$ & - \\
\hline RS22(45)-RL2(147) & 2 & & & & & 1 & 1 & $1.5 \mathrm{E}-04$ & - \\
\hline RPOC(2)-RS1(363) & 2 & & 1 & 1 & & & & $1.6 \mathrm{E}-08$ & - \\
\hline RL31(70)-RL5(120) & 2 & & & & & 1 & 1 & $1.7 \mathrm{E}-10$ & - \\
\hline \multicolumn{10}{|l|}{ Intra Molecular } \\
\hline RL7(71)-RL7(85) & 192 & 31 & 34 & 66 & 22 & 19 & 20 & $3.1 \mathrm{E}-09$ & - \\
\hline RL1(141)-RL1(167) & 190 & 38 & 35 & 41 & 33 & 18 & 25 & $2.0 \mathrm{E}-23$ & - \\
\hline RL6(6)-RL6(44) & 138 & 20 & 17 & 27 & 19 & 29 & 26 & $2.7 \mathrm{E}-25$ & 10.8 \\
\hline RS3(86)-RS3(89) & 125 & 20 & 22 & 22 & 19 & 20 & 22 & $3.7 \mathrm{E}-09$ & 5.6 \\
\hline RL22(1)-RL22(73) & 105 & 19 & 18 & 19 & 17 & 17 & 15 & $1.4 \mathrm{E}-12$ & 12.7 \\
\hline RS4(151)-RS4(156) & 102 & 15 & 19 & 21 & 18 & 17 & 12 & $1.4 \mathrm{E}-06$ & 5.1 \\
\hline RL22(6)-RL22(73) & 90 & 7 & 12 & 35 & 13 & 11 & 12 & $1.4 \mathrm{E}-21$ & 11.3 \\
\hline RS20(34)-RS20(49) & 88 & 13 & 12 & 13 & 10 & 24 & 16 & $7.0 \mathrm{E}-24$ & 9.6 \\
\hline RL18(63)-RL18(76) & 82 & 12 & 12 & 18 & 11 & 15 & 14 & $8.6 \mathrm{E}-13$ & 16.2 \\
\hline RL2(97)-RL2(125) & 78 & 15 & 12 & 22 & 12 & 6 & 11 & $5.2 \mathrm{E}-25$ & 18.2 \\
\hline RL1(141)-RL1(198) & 78 & 10 & 9 & 24 & 15 & 10 & 10 & $2.3 \mathrm{E}-18$ & - \\
\hline RS3(108)-RS3(219) & 62 & 10 & 10 & 13 & 9 & 10 & 10 & $5.6 \mathrm{E}-15$ & - \\
\hline RL7(101)-RL7(109) & 56 & 12 & 15 & 8 & 6 & 8 & 7 & $1.8 \mathrm{E}-16$ & - \\
\hline RL7(71)-RL7(82) & 50 & 9 & 8 & 11 & 7 & 9 & 6 & $3.3 \mathrm{E}-15$ & - \\
\hline RL7(66)-RL7(85) & 50 & 7 & 10 & 9 & 7 & 9 & 8 & $1.2 \mathrm{E}-08$ & - \\
\hline RL11(81)-RL11(87) & 49 & 9 & 10 & 10 & 9 & 4 & 7 & $2.7 \mathrm{E}-14$ & 4.8 \\
\hline RS3(108)-RS3(147) & 49 & 8 & 9 & 11 & 7 & 6 & 8 & $1.3 \mathrm{E}-08$ & 13.9 \\
\hline RL5(47)-RL5(64) & 49 & 5 & 11 & 7 & 9 & 10 & 7 & $1.3 \mathrm{E}-15$ & 18.1 \\
\hline RL10(37)-RL10(105) & 49 & 7 & 8 & 9 & 8 & 9 & 8 & $1.0 \mathrm{E}-07$ & - \\
\hline RL5(47)-RL5(69) & 48 & 10 & 8 & 6 & 14 & 5 & 5 & $2.6 \mathrm{E}-17$ & 12.4 \\
\hline
\end{tabular}




\begin{tabular}{|c|c|c|c|c|c|c|c|c|c|}
\hline RS4(83)-RS4(185) & 48 & 7 & 9 & 2 & 9 & 12 & 9 & $2.8 \mathrm{E}-07$ & 14.8 \\
\hline RS3(49)-RS3(79) & 46 & 9 & 6 & 7 & 9 & 7 & 8 & $1.5 \mathrm{E}-12$ & 16.5 \\
\hline RL1(54)-RL1(205) & 44 & 6 & 7 & 11 & 9 & 9 & 2 & $3.0 \mathrm{E}-16$ & - \\
\hline RL2(183)-RL2(265) & 41 & 6 & 6 & 5 & 12 & 4 & 8 & $2.8 \mathrm{E}-10$ & 8.3 \\
\hline RL2(108)-RL2(125) & 41 & 10 & 9 & 4 & 9 & 5 & 4 & $5.9 \mathrm{E}-11$ & 10.7 \\
\hline RS12(108)-RS12(111) & 39 & 6 & 7 & 4 & 8 & 8 & 6 & $1.4 \mathrm{E}-09$ & 8.9 \\
\hline RL22(42)-RL22(49) & 38 & 15 & 12 & 4 & 7 & & & $2.2 \mathrm{E}-12$ & 11.2 \\
\hline RS3(80)-RS3(89) & 37 & 5 & 5 & 5 & 5 & 8 & 9 & $5.4 \mathrm{E}-13$ & 15.6 \\
\hline RL9(35)-RL9(42) & 37 & 9 & 5 & & & 11 & 12 & $2.5 \mathrm{E}-17$ & 17.6 \\
\hline RL1(154)-RL1(184) & 36 & 4 & 4 & 8 & 7 & 5 & 8 & $6.5 \mathrm{E}-20$ & - \\
\hline RS4(167)-RS4(183) & 34 & 2 & 5 & 3 & 4 & 10 & 10 & $2.0 \mathrm{E}-06$ & 12.8 \\
\hline RS10(1)-RS10(82) & 34 & 7 & 8 & 7 & 6 & 4 & 2 & $2.0 \mathrm{E}-07$ & - \\
\hline RL1(197)-RL1(200) & 33 & 8 & 8 & 3 & 8 & 3 & 3 & $1.5 \mathrm{E}-17$ & - \\
\hline RL16(118)-RL16(127) & 32 & 6 & 7 & 1 & 1 & 9 & 8 & $1.3 \mathrm{E}-09$ & 11.2 \\
\hline RL22(1)-RL22(70) & 32 & 9 & 5 & 5 & 5 & 3 & 5 & $3.5 \mathrm{E}-14$ & 11.4 \\
\hline $\mathrm{RS} 3(38)-\mathrm{RS} 3(45)$ & 30 & 5 & 4 & 10 & 4 & 4 & 3 & $1.1 \mathrm{E}-11$ & 10.7 \\
\hline RL25(10)-RL25(25) & 30 & 4 & 7 & 4 & 3 & 6 & 6 & $2.8 \mathrm{E}-06$ & 13.5 \\
\hline $\operatorname{RL7}(66)-R L 7(82)$ & 30 & 5 & 5 & 6 & 5 & 5 & 4 & $3.2 \mathrm{E}-16$ & - \\
\hline RL9(1)-RL9(22) & 29 & 4 & 5 & 5 & 5 & 5 & 5 & $1.1 \mathrm{E}-24$ & 6.0 \\
\hline RL11(51)-RL11(82) & 29 & 8 & 9 & 6 & 6 & & & $9.7 \mathrm{E}-06$ & 12.0 \\
\hline RL9(42)-RL9(57) & 28 & 5 & 6 & 2 & 6 & 5 & 4 & $1.3 \mathrm{E}-22$ & 21.3 \\
\hline RL7(85)-RL7(96) & 28 & 9 & 7 & 5 & 6 & 1 & & $1.3 \mathrm{E}-15$ & - \\
\hline RL4(6)-RL4(137) & 26 & 3 & 5 & 4 & 6 & 4 & 4 & 4.7E-06 & 14.5 \\
\hline RL7(96)-RL7(108) & 26 & 5 & 5 & 3 & 3 & 5 & 5 & $7.0 \mathrm{E}-12$ & - \\
\hline RL29(44)-RL29(54) & 25 & 9 & 10 & & & 2 & 4 & $7.1 \mathrm{E}-27$ & 15.7 \\
\hline RL4(1)-RL4(137) & 25 & 2 & 1 & 9 & 2 & 5 & 6 & $3.9 \mathrm{E}-08$ & 28.4 \\
\hline RS3(45)-RS3(89) & 24 & 8 & 7 & 5 & 4 & & & $2.0 \mathrm{E}-06$ & 15.8 \\
\hline RL3(1)-RL3(204) & 23 & 4 & 5 & & 4 & 4 & 6 & $1.2 \mathrm{E}-13$ & 7.8 \\
\hline RL16(62)-RL16(133) & 23 & 4 & 4 & & 4 & 5 & 6 & $1.2 \mathrm{E}-06$ & 14.5 \\
\hline RS5(156)-RS5(159) & 23 & 10 & 8 & & & & 5 & $3.4 \mathrm{E}-29$ & - \\
\hline RS2(64)-RS2(115) & 22 & 6 & 5 & & 4 & & 7 & $2.4 \mathrm{E}-19$ & 24.1 \\
\hline RL9(35)-RL9(112) & 22 & 4 & 5 & 10 & & 3 & & $1.6 \mathrm{E}-14$ & 42.5 \\
\hline RL20(85)-RL20(112) & 21 & 7 & 4 & 1 & 3 & 2 & 4 & $6.4 \mathrm{E}-14$ & 9.7 \\
\hline RS3(45)-RS3(86) & 21 & 8 & 8 & & 5 & & & $4.0 \mathrm{E}-12$ & 12.3 \\
\hline RS20(16)-RS20(19) & 20 & & 3 & & & 9 & 8 & $4.8 \mathrm{E}-08$ & 5.9 \\
\hline RL25(25)-RL25(46) & 20 & 5 & 5 & 5 & 5 & & & $8.6 \mathrm{E}-13$ & 9.4 \\
\hline RS12(108)-RS12(120) & 20 & 2 & 3 & 1 & 4 & 6 & 4 & $7.1 \mathrm{E}-15$ & 9.6 \\
\hline RS21(40)-RS21(49) & 20 & 7 & 7 & & & 3 & 3 & $9.2 \mathrm{E}-10$ & 14.4 \\
\hline RS4(77)-RS4(83) & 19 & 9 & 10 & & & & & $1.7 \mathrm{E}-09$ & 11.8 \\
\hline RL14(59)-RL14(111) & 19 & 4 & 4 & & 3 & 2 & 6 & $1.6 \mathrm{E}-06$ & 13.6 \\
\hline RS9(2)-RS9(22) & 18 & 1 & 2 & 6 & 3 & 3 & 3 & $4.5 \mathrm{E}-07$ & - \\
\hline RS18(9)-RS18(30) & 18 & 4 & 2 & 1 & 6 & 1 & 4 & $5.5 \mathrm{E}-07$ & - \\
\hline YggL(87)-YggL(93) & 18 & 5 & 3 & & 4 & 2 & 4 & $9.1 \mathrm{E}-09$ & - \\
\hline RS2(132)-RS2(143) & 17 & 4 & 4 & & & 5 & 4 & $1.2 \mathrm{E}-13$ & 17.1 \\
\hline RL7(66)-RL7(96) & 17 & 4 & 4 & 4 & 4 & 1 & & $4.8 \mathrm{E}-11$ & - \\
\hline RL9(8)-RL9(35) & 16 & & & 7 & & 5 & 4 & $3.2 \mathrm{E}-08$ & 8.0 \\
\hline RL29(44)-RL29(60) & 16 & 9 & 7 & & & & & $5.6 \mathrm{E}-12$ & 23.9 \\
\hline RL9(35)-RL9(57) & 16 & 3 & 1 & 7 & 5 & & & $1.7 \mathrm{E}-17$ & 25.3 \\
\hline
\end{tabular}




\begin{tabular}{|c|c|c|c|c|c|c|c|c|c|}
\hline RS9(2)-RS9(13) & 16 & 3 & 2 & 1 & 1 & 4 & 5 & $2.9 \mathrm{E}-06$ & - \\
\hline RL25(10)-RL25(68) & 15 & & 4 & 2 & 4 & 3 & 2 & $6.3 \mathrm{E}-11$ & 17.6 \\
\hline RS1(279)-RS1(347) & 15 & 2 & 3 & 4 & & 4 & 2 & $8.7 \mathrm{E}-13$ & - \\
\hline RS2(2)-RS2(59) & 15 & & 4 & 4 & 4 & 1 & 2 & $9.3 \mathrm{E}-10$ & - \\
\hline RL18(76)-RL18(88) & 14 & 4 & 4 & & & 2 & 4 & $1.5 \mathrm{E}-08$ & 13.3 \\
\hline RL9(22)-RL9(35) & 14 & 4 & 6 & & & 3 & 1 & $3.6 \mathrm{E}-11$ & 14.3 \\
\hline RL24(91)-RL24(104) & 14 & & 4 & & 4 & 3 & 3 & $2.3 \mathrm{E}-08$ & - \\
\hline RS1(450)-RS1(528) & 14 & 4 & 9 & 1 & & & & $4.1 \mathrm{E}-15$ & - \\
\hline RS3(108)-RS3(225) & 14 & 3 & 5 & 4 & 2 & & & $2.6 \mathrm{E}-18$ & - \\
\hline RL20(78)-RL20(85) & 13 & 4 & 4 & & & 3 & 2 & $1.8 \mathrm{E}-12$ & 10.0 \\
\hline RS2(128)-RS2(132) & 12 & 2 & 4 & & & 5 & 1 & $9.7 \mathrm{E}-11$ & 8.1 \\
\hline RL2(68)-RL2(71) & 12 & 2 & 3 & & & 4 & 3 & $1.3 \mathrm{E}-09$ & 9.5 \\
\hline RL28(54)-RL28(62) & 12 & 4 & 4 & & 2 & & 2 & $1.1 \mathrm{E}-05$ & 11.4 \\
\hline RL7(71)-RL7(96) & 12 & 5 & 2 & & 2 & 2 & 1 & $1.1 \mathrm{E}-05$ & - \\
\hline RL4(123)-RL4(137) & 11 & 1 & 3 & 5 & 1 & & 1 & $2.9 \mathrm{E}-05$ & 12.7 \\
\hline RL3(1)-RL3(208) & 11 & 3 & 3 & & & 3 & 2 & $5.1 \mathrm{E}-17$ & 13.1 \\
\hline RS2(66)-RS2(115) & 11 & 6 & 3 & 2 & & & & $2.2 \mathrm{E}-19$ & 20.1 \\
\hline RL31(62)-RL31(70) & 11 & 1 & 2 & & & 3 & 5 & $1.3 \mathrm{E}-07$ & - \\
\hline RL7(82)-RL7(96) & 11 & 1 & 3 & 3 & 4 & & & $5.1 \mathrm{E}-09$ & - \\
\hline RS1(150)-RS1(247) & 11 & 6 & 3 & & 1 & & 1 & $1.5 \mathrm{E}-04$ & - \\
\hline RL22(28)-RL22(70) & 10 & & 2 & 6 & 2 & & & $9.6 \mathrm{E}-21$ & 3.9 \\
\hline RL28(54)-RL28(61) & 10 & 3 & 3 & & & 1 & 3 & $2.4 \mathrm{E}-05$ & 9.8 \\
\hline RL3(1)-RL3(8) & 10 & 3 & 3 & & & 1 & 3 & $2.3 \mathrm{E}-07$ & 21.3 \\
\hline RS6(93)-RS6(106) & 10 & 1 & 4 & 3 & 2 & & & $1.0 \mathrm{E}-06$ & - \\
\hline RS4(183)-RS4(185) & 9 & 1 & 6 & & & 1 & 1 & $3.5 \mathrm{E}-05$ & 7.2 \\
\hline RL15(129)-RL15(141) & 9 & 3 & 3 & & & 1 & 2 & $5.7 \mathrm{E}-07$ & 13.6 \\
\hline RS15(71)-RS15(73) & 8 & 2 & 1 & & & 4 & 1 & $2.1 \mathrm{E}-08$ & 5.5 \\
\hline RS4(156)-RS4(167) & 8 & 4 & 4 & & & & & $1.8 \mathrm{E}-19$ & 16.5 \\
\hline RS2(112)-RS2(115) & 7 & 1 & 2 & 1 & & 2 & 1 & $6.3 \mathrm{E}-05$ & 5.0 \\
\hline RS20(71)-RS20(85) & 7 & 2 & 3 & & & 1 & 1 & $1.0 \mathrm{E}-05$ & 21.2 \\
\hline ATPA(384)-ATPA(388) & 7 & 1 & 3 & & 3 & & & $1.2 \mathrm{E}-13$ & - \\
\hline RS1(272)-RS1(279) & 7 & 4 & 2 & & & 1 & & 4.4E-10 & - \\
\hline RS1(247)-RS1(260) & 7 & 3 & 1 & 1 & 1 & & 1 & $1.1 \mathrm{E}-04$ & - \\
\hline RS9(2)-RS9(27) & 7 & 2 & 2 & 1 & & 1 & 1 & $4.5 \mathrm{E}-09$ & - \\
\hline RL33(33)-RL33(50) & 6 & & & 2 & 4 & & & $8.0 \mathrm{E}-07$ & 7.1 \\
\hline RS10(30)-RS10(82) & 6 & & & 1 & & & 5 & $7.9 \mathrm{E}-14$ & 10.4 \\
\hline RL4(6)-RL4(185) & 6 & & & 1 & 4 & 1 & & $2.1 \mathrm{E}-10$ & 12.4 \\
\hline RS3(108)-RS3(226) & 6 & 3 & 3 & & & & & $9.9 \mathrm{E}-06$ & - \\
\hline RS19(28)-RS19(29) & 5 & 1 & 1 & & 1 & 1 & 1 & $2.2 \mathrm{E}-06$ & 3.8 \\
\hline RL11(72)-RL11(82) & 5 & 1 & 2 & & & 1 & 1 & $1.1 \mathrm{E}-04$ & 18.9 \\
\hline RS3(49)-RS3(80) & 5 & 3 & 2 & & & & & $2.8 \mathrm{E}-09$ & 19.1 \\
\hline RS11(2)-RS11(75) & 5 & 1 & 1 & & & 1 & 2 & $4.7 \mathrm{E}-05$ & - \\
\hline RL7(2)-RL7(71) & 5 & 1 & 1 & & 3 & & & $3.4 \mathrm{E}-07$ & - \\
\hline RL1(141)-RL1(157) & 5 & & 4 & & 1 & & & $7.3 \mathrm{E}-08$ & - \\
\hline RS3(49)-RS3(86) & 4 & & & & 1 & 1 & 2 & $2.8 \mathrm{E}-06$ & 14.5 \\
\hline RS12(44)-RS12(51) & 4 & 2 & 2 & & & & & $1.9 \mathrm{E}-05$ & 17.1 \\
\hline RS21(25)-RS21(40) & 4 & & 1 & & & & 3 & $7.5 \mathrm{E}-13$ & 22.0 \\
\hline RL1(54)-RL1(141) & 4 & & 2 & 2 & & & & $5.5 \mathrm{E}-06$ & - \\
\hline
\end{tabular}




\begin{tabular}{|c|c|c|c|c|c|c|c|c|c|}
\hline RL17(99)-RL17(121) & 4 & 1 & & & & 3 & & $3.9 \mathrm{E}-04$ & - \\
\hline RL7(101)-RL7(121) & 4 & 1 & 3 & & & & & $3.0 \mathrm{E}-17$ & - \\
\hline RL24(43)-RL24(47) & 3 & 1 & 1 & & & & 1 & $2.1 \mathrm{E}-18$ & 12.1 \\
\hline RL27(19)-RL27(24) & 3 & 1 & 2 & & & & & $7.4 \mathrm{E}-12$ & 13.4 \\
\hline RL3(7)-RL3(56) & 3 & & 1 & & & & 2 & $1.6 \mathrm{E}-07$ & 17.6 \\
\hline RS20(16)-RS20(71) & 3 & & 2 & & & & 1 & $3.1 \mathrm{E}-07$ & 24.0 \\
\hline RL1(157)-RL1(184) & 3 & 1 & 2 & & & & & $5.6 \mathrm{E}-10$ & - \\
\hline RS3(219)-RS3(226) & 3 & & 2 & 1 & & & & $7.0 \mathrm{E}-05$ & - \\
\hline DnaK(246)-DnaK(304) & 3 & 2 & & & 1 & & & $4.9 \mathrm{E}-06$ & - \\
\hline RL7(60)-RL7(85) & 2 & 1 & 1 & & & & & $2.6 \mathrm{E}-10$ & - \\
\hline RL7(96)-RL7(101) & 2 & 1 & 1 & & & & & $1.2 \mathrm{E}-10$ & - \\
\hline RL7(2)-RL7(96) & 2 & & 1 & & & 1 & & $1.1 \mathrm{E}-04$ & - \\
\hline \multicolumn{10}{|c|}{ EGS } \\
\hline Site-pairs & \#total spec & \#Exp1 & \#Exp2 & \#Exp3 & \#Exp4 & \#Exp5 & \#Exp6 & Best E_value & $\begin{array}{c}\mathrm{Ca}-\mathrm{Ca} \\
\text { distance }\end{array}$ \\
\hline \multicolumn{10}{|l|}{ Inter Molecular } \\
\hline RS7(11)-RS9(100) & 152 & 31 & 27 & 15 & 15 & 32 & 32 & $3.0 \mathrm{E}-13$ & 14.3 \\
\hline RL32(32)-RL17(121) & 142 & 30 & 34 & 12 & 18 & 21 & 27 & $9.8 \mathrm{E}-10$ & - \\
\hline RL9(42)-RL28(44) & 107 & 22 & 25 & 15 & 22 & 11 & 12 & 3.0E-11 & 21.2 \\
\hline RS21(5)-RS18(30) & 90 & 18 & 19 & 8 & 12 & 17 & 16 & $2.5 \mathrm{E}-08$ & - \\
\hline RS17(16)-RS15(71) & 75 & 15 & 15 & 4 & 5 & 20 & 16 & $2.2 \mathrm{E}-19$ & 24.6 \\
\hline RS8(22)-RS2(28) & 70 & 16 & 14 & 2 & 7 & 16 & 15 & $3.4 \mathrm{E}-17$ & 12.5 \\
\hline RL18(17)-RL27(62) & 64 & 8 & 10 & 10 & 9 & 12 & 15 & $1.3 \mathrm{E}-20$ & 15.2 \\
\hline RS18(9)-RS21(25) & 57 & 11 & 13 & 3 & 8 & 11 & 11 & $6.3 E-06$ & - \\
\hline RL9(35)-RL28(44) & 52 & 9 & 10 & 8 & 6 & 11 & 8 & $4.0 \mathrm{E}-07$ & 15.9 \\
\hline RL9(112)-RL2(183) & 48 & 6 & 8 & 6 & 9 & 9 & 10 & $2.2 \mathrm{E}-10$ & 72.2 \\
\hline RS15(47)-RL2(108) & 46 & & 9 & 14 & 5 & 5 & 13 & $1.4 \mathrm{E}-10$ & 23.0 \\
\hline RS19(28)-RL31(70) & 44 & 9 & 8 & 7 & 8 & 4 & 8 & $4.1 \mathrm{E}-12$ & - \\
\hline RS21(25)-RS18(30) & 38 & & & 8 & 14 & 8 & 8 & $1.9 \mathrm{E}-19$ & 22.8 \\
\hline RS14(7)-RS3(38) & 36 & 7 & 5 & 3 & 4 & 8 & 9 & $1.3 \mathrm{E}-08$ & 17.7 \\
\hline RL9(89)-RL2(183) & 32 & 4 & 5 & 8 & 7 & 4 & 4 & $6.6 \mathrm{E}-15$ & 88.7 \\
\hline RL23(9)-RL29(54) & 30 & 5 & 7 & 3 & 3 & 5 & 7 & $4.0 \mathrm{E}-06$ & 16.2 \\
\hline RS19(17)-RL31(70) & 28 & & 5 & 10 & & 8 & 5 & $6.3 \mathrm{E}-08$ & - \\
\hline RL5(78)-RS13(103) & 28 & 4 & 3 & 2 & 4 & 7 & 8 & $6.1 \mathrm{E}-07$ & 38.5 \\
\hline RL28(44)-RL9(57) & 27 & 4 & 5 & 3 & 8 & 3 & 4 & $4.5 \mathrm{E}-14$ & 34.6 \\
\hline RS8(89)-RS5(167) & 25 & 4 & 4 & 4 & 4 & 5 & 4 & 4.4E-11 & - \\
\hline RL25(83)-RL16(127) & 24 & 5 & 7 & & & 8 & 4 & $1.3 \mathrm{E}-07$ & 12.6 \\
\hline RS2(128)-RS3(147) & 24 & 7 & 5 & 4 & 2 & 2 & 4 & $4.3 \mathrm{E}-08$ & 26.1 \\
\hline RL10(37)-RL11(72) & 22 & 1 & 3 & 9 & & 7 & 2 & $1.3 \mathrm{E}-07$ & - \\
\hline RS13(78)-RL5(120) & 20 & 3 & 3 & 2 & 4 & 4 & 4 & $9.7 \mathrm{E}-10$ & 23.4 \\
\hline RS4(83)-RS5(167) & 20 & 2 & 5 & & 4 & 5 & 4 & $6.3 \mathrm{E}-17$ & - \\
\hline RS14(47)-RL31(70) & 19 & 5 & & 3 & 6 & & 5 & $1.4 \mathrm{E}-08$ & - \\
\hline RL6(29)-RL7(71) & 17 & 5 & 7 & 1 & 1 & 1 & 2 & 7.1E-10 & - \\
\hline RS8(94)-RS5(167) & 16 & & 1 & & 7 & 5 & 3 & $4.9 \mathrm{E}-11$ & - \\
\hline RS10(1)-RS3(62) & 15 & 3 & 4 & 3 & 5 & & & $1.9 \mathrm{E}-08$ & - \\
\hline RS13(103)-RL5(120) & 15 & 1 & & 2 & 2 & 6 & 4 & $1.1 \mathrm{E}-06$ & 46.9 \\
\hline RS3(108)-RS1(464) & 14 & & 2 & 5 & 3 & 2 & 2 & $3.1 \mathrm{E}-07$ & - \\
\hline RS19(28)-RL5(120) & 12 & 5 & & 2 & 3 & & 2 & $2.5 \mathrm{E}-11$ & 24.3 \\
\hline
\end{tabular}




\begin{tabular}{|c|c|c|c|c|c|c|c|c|c|}
\hline RL32(53)-RL17(121) & 11 & & 2 & 2 & 2 & 3 & 2 & $1.4 \mathrm{E}-05$ & - \\
\hline $\operatorname{RL7}(71)-R L 6(86)$ & 10 & 1 & 4 & 4 & 1 & & & $1.1 \mathrm{E}-06$ & - \\
\hline RL17(35)-RL32(53) & 9 & & & 2 & & 3 & 4 & $3.4 \mathrm{E}-08$ & 16.3 \\
\hline RS18(30)-RS6(104) & 7 & 5 & 2 & & & & & $8.2 \mathrm{E}-16$ & - \\
\hline RL28(44)-RL9(112) & 7 & & 4 & & 2 & & 1 & $1.2 \mathrm{E}-06$ & 50.9 \\
\hline RS19(17)-RS14(47) & 7 & & & 4 & & 2 & 1 & $2.4 \mathrm{E}-12$ & 12.0 \\
\hline RS2(81)-RS3(108) & 6 & & & 2 & & 3 & 1 & $1.8 \mathrm{E}-09$ & 53.4 \\
\hline RL18(1)-RL5(161) & 6 & & & 1 & 1 & & 4 & $5.0 \mathrm{E}-05$ & - \\
\hline RS17(43)-RL27(78) & 6 & & 1 & & & 1 & 4 & $1.1 \mathrm{E}-06$ & 158.3 \\
\hline RL1(14)-RL9(112) & 6 & 3 & 1 & 1 & & 1 & & $1.7 \mathrm{E}-06$ & - \\
\hline RS2(105)-GlpD(302) & 6 & 3 & & & & 2 & 1 & $7.4 \mathrm{E}-05$ & - \\
\hline RL31(70)-RL5(120) & 6 & & & 4 & 2 & & & $3.3 \mathrm{E}-10$ & - \\
\hline RS18(30)-RS6(106) & 5 & 2 & 3 & & & & & $3.5 \mathrm{E}-04$ & - \\
\hline RS12(108)-GapA(225) & 5 & & 1 & 2 & 2 & & & $8.9 \mathrm{E}-07$ & - \\
\hline RL22(28)-RL32(37) & 5 & 3 & 2 & & & & & $3.7 \mathrm{E}-06$ & 16.8 \\
\hline RL31(8)-RL5(145) & 5 & & & 4 & 1 & & & $1.2 \mathrm{E}-09$ & - \\
\hline RL31(70)-CRL(14) & 4 & 1 & 1 & & 1 & 1 & & $6.8 \mathrm{E}-05$ & - \\
\hline RS12(108)-СyoA(221) & 4 & & & 2 & & 1 & 1 & $6.0 \mathrm{E}-07$ & - \\
\hline RS15(71)-RS17(81) & 4 & 2 & & 1 & & 1 & & $1.5 \mathrm{E}-04$ & - \\
\hline RS11(1)-RL21(81) & 4 & & & & & 2 & 2 & $3.3 \mathrm{E}-05$ & - \\
\hline RL9(57)-RS4(185) & 4 & & & & & 1 & 3 & $2.6 \mathrm{E}-05$ & 66.7 \\
\hline RS8(69)-RS5(86) & 4 & & & & & 3 & 1 & $1.6 \mathrm{E}-09$ & 19.5 \\
\hline RS21(5)-RS11(80) & 3 & 1 & & 2 & & & & $7.9 \mathrm{E}-05$ & - \\
\hline $\mathrm{CH} 10(13)-\operatorname{Sec} Y(20)$ & 2 & 1 & & & 1 & & & $3.8 \mathrm{E}-05$ & - \\
\hline $\operatorname{RS} 12(2)-\operatorname{RS} 3(27)$ & 2 & 1 & 1 & & & & & $1.1 \mathrm{E}-04$ & 102.9 \\
\hline $\operatorname{RL7}(85)-R L 6(86)$ & 2 & 1 & 1 & & & & & $4.2 \mathrm{E}-14$ & - \\
\hline RS14(23)-RL31(70) & 2 & & 1 & & & 1 & & $2.8 \mathrm{E}-06$ & - \\
\hline RL27(4)-RS21(54) & 2 & & 1 & & & & 1 & $1.4 \mathrm{E}-04$ & - \\
\hline RS3(89)-RL17(121) & 2 & & & & & 1 & 1 & $5.2 \mathrm{E}-04$ & - \\
\hline Intra Molecular & & & & & & & & & \\
\hline RS20(34)-RS20(49) & 321 & 60 & 63 & 12 & 27 & 78 & 81 & $6.1 \mathrm{E}-23$ & 9.6 \\
\hline RS4(151)-RS4(156) & 303 & 74 & 70 & 16 & 15 & 59 & 69 & $3.2 \mathrm{E}-07$ & 5.1 \\
\hline RL2(97)-RL2(125) & 239 & 49 & 68 & 21 & 34 & 31 & 36 & $1.3 \mathrm{E}-23$ & 18.2 \\
\hline RL20(78)-RL20(85) & 193 & 32 & 33 & 26 & 28 & 37 & 37 & $4.0 \mathrm{E}-17$ & 10.0 \\
\hline RL18(63)-RL18(76) & 172 & 38 & 23 & 15 & 20 & 41 & 35 & $7.3 \mathrm{E}-15$ & 16.2 \\
\hline RL2(183)-RL2(265) & 167 & 30 & 24 & 20 & 16 & 37 & 40 & $3.5 \mathrm{E}-13$ & 8.3 \\
\hline RL1(54)-RL1(167) & 164 & 27 & 34 & 25 & 22 & 32 & 24 & $6.4 \mathrm{E}-21$ & - \\
\hline RS4(83)-RS4(185) & 161 & 22 & 26 & 13 & 30 & 34 & 36 & 4.4E-07 & 14.8 \\
\hline RS9(2)-RS9(60) & 160 & 36 & 37 & 10 & 11 & 34 & 32 & $1.3 \mathrm{E}-19$ & - \\
\hline RL6(6)-RL6(44) & 126 & 27 & 27 & 13 & 29 & 14 & 16 & $1.8 \mathrm{E}-26$ & 10.8 \\
\hline RL22(6)-RL22(73) & 118 & 27 & 27 & 15 & 14 & 16 & 19 & $5.7 \mathrm{E}-20$ & 11.3 \\
\hline RL22(1)-RL22(73) & 115 & 15 & 25 & 14 & 16 & 23 & 22 & $2.8 \mathrm{E}-12$ & 12.7 \\
\hline RL31(62)-RL31(70) & 115 & 28 & 14 & 12 & 12 & 28 & 21 & $2.5 \mathrm{E}-09$ & - \\
\hline RS20(16)-RS20(19) & 111 & 4 & & 8 & 8 & 44 & 47 & $1.2 \mathrm{E}-09$ & 5.9 \\
\hline RL2(108)-RL2(125) & 106 & 20 & 20 & 10 & 23 & 17 & 16 & $5.8 \mathrm{E}-10$ & 10.7 \\
\hline RL18(76)-RL18(88) & 100 & 21 & 18 & 10 & 10 & 21 & 20 & $5.0 \mathrm{E}-10$ & 13.3 \\
\hline RL9(42)-RL9(57) & 88 & 37 & 11 & 7 & 7 & 16 & 10 & $6.6 \mathrm{E}-24$ & 21.3 \\
\hline RS9(2)-RS9(27) & 85 & 15 & 13 & 10 & 9 & 18 & 20 & $1.8 \mathrm{E}-18$ & - \\
\hline
\end{tabular}




\begin{tabular}{|c|c|c|c|c|c|c|c|c|c|}
\hline RS7(114)-RS7(131) & 77 & 18 & 18 & 9 & 7 & 12 & 13 & $1.3 \mathrm{E}-09$ & 18.9 \\
\hline RS12(108)-RS12(111) & 71 & 14 & 16 & 4 & 5 & 16 & 16 & $4.8 \mathrm{E}-09$ & 8.9 \\
\hline RL19(63)-RL19(87) & 56 & 7 & 11 & 9 & 8 & 12 & 9 & $1.1 \mathrm{E}-26$ & 12.9 \\
\hline RL1(54)-RL1(205) & 52 & 10 & 11 & 12 & 9 & 6 & 4 & $3.0 \mathrm{E}-16$ & - \\
\hline RS9(2)-RS9(22) & 46 & 10 & 9 & 5 & 4 & 8 & 10 & $2.2 \mathrm{E}-11$ & - \\
\hline RS10(1)-RS10(82) & 45 & 9 & 9 & 8 & 5 & 6 & 8 & $4.5 \mathrm{E}-07$ & - \\
\hline RL24(26)-RL24(61) & 44 & 4 & 14 & & & 11 & 15 & $1.1 \mathrm{E}-09$ & 11.4 \\
\hline RS13(78)-RS13(103) & 43 & 2 & 4 & 6 & 5 & 13 & 13 & $3.4 \mathrm{E}-08$ & 26.3 \\
\hline YggL(87)-YggL(93) & 41 & 4 & 6 & 6 & 6 & 9 & 10 & $3.7 \mathrm{E}-08$ & - \\
\hline RS12(108)-RS12(120) & 41 & 11 & 8 & 6 & 7 & 3 & 6 & $5.1 \mathrm{E}-13$ & 9.6 \\
\hline $\operatorname{RL7}(71)-R L 7(85)$ & 41 & 14 & 13 & 4 & 4 & 2 & 4 & $1.2 \mathrm{E}-07$ & - \\
\hline RS3(86)-RS3(89) & 39 & 13 & 6 & 10 & 4 & 4 & 2 & $1.7 \mathrm{E}-06$ & 5.6 \\
\hline RL24(91)-RL24(104) & 38 & 5 & 8 & 3 & 3 & 8 & 11 & $1.4 \mathrm{E}-07$ & - \\
\hline RS19(18)-RS19(29) & 37 & 12 & 12 & 8 & 5 & & & $1.7 \mathrm{E}-05$ & 11.8 \\
\hline RL22(28)-RL22(70) & 36 & 11 & 11 & 5 & & 4 & 5 & $3.9 \mathrm{E}-25$ & 3.9 \\
\hline RS3(38)-RS3(62) & 35 & 10 & 3 & 8 & 2 & 6 & 6 & 3.7E-16 & 16.6 \\
\hline RL22(1)-RL22(70) & 34 & 10 & 9 & 6 & 1 & 3 & 5 & $3.0 \mathrm{E}-15$ & 11.4 \\
\hline RL16(62)-RL16(133) & 33 & 2 & 4 & 3 & 6 & 11 & 7 & $1.1 \mathrm{E}-07$ & 14.5 \\
\hline $\operatorname{RS} 3(38)-\operatorname{RS} 3(45)$ & 32 & 7 & 9 & 4 & 5 & 4 & 3 & $3.9 \mathrm{E}-13$ & 10.7 \\
\hline RL9(35)-RL9(112) & 31 & 4 & & 6 & 5 & 7 & 9 & $1.0 \mathrm{E}-20$ & 42.5 \\
\hline RL15(129)-RL15(141) & 30 & 8 & 3 & 3 & 3 & 6 & 7 & $1.2 \mathrm{E}-06$ & 13.6 \\
\hline RS10(30)-RS10(82) & 27 & 5 & 7 & 5 & & 5 & 5 & $5.2 \mathrm{E}-23$ & 10.4 \\
\hline RL16(118)-RL16(127) & 27 & & 3 & 8 & 4 & 7 & 5 & $3.0 \mathrm{E}-09$ & 11.2 \\
\hline RL1(54)-RL1(210) & 27 & 8 & 6 & 6 & 5 & 1 & 1 & $4.9 \mathrm{E}-09$ & - \\
\hline RS4(167)-RS4(183) & 26 & 5 & 8 & 3 & 1 & 6 & 3 & $7.8 \mathrm{E}-06$ & 12.8 \\
\hline RL3(55)-RL3(62) & 26 & 3 & 2 & 3 & 4 & 6 & 8 & $2.8 \mathrm{E}-14$ & 13.3 \\
\hline RL7(101)-RL7(109) & 25 & 11 & 8 & 2 & 4 & & & $2.9 \mathrm{E}-10$ & - \\
\hline RS16(46)-RS16(80) & 23 & 3 & 1 & 2 & 8 & 6 & 3 & $1.9 \mathrm{E}-05$ & 19.3 \\
\hline RS19(21)-RS19(29) & 22 & 4 & 10 & 1 & 7 & & & $2.1 \mathrm{E}-06$ & 13.2 \\
\hline RS3(45)-RS3(89) & 22 & 7 & 5 & 6 & & & 4 & $2.1 \mathrm{E}-07$ & 15.8 \\
\hline RL5(47)-RL5(69) & 19 & 4 & 1 & 5 & 5 & 4 & & $1.1 \mathrm{E}-12$ & 12.4 \\
\hline RS7(56)-RS7(131) & 17 & 5 & 11 & & 1 & & & $1.5 \mathrm{E}-10$ & 17.3 \\
\hline RL5(2)-RL5(15) & 17 & 6 & 6 & 3 & & 2 & & $1.4 \mathrm{E}-16$ & 18.6 \\
\hline RL5(47)-RL5(64) & 16 & 8 & 1 & 4 & 1 & 2 & & $2.9 \mathrm{E}-11$ & 18.1 \\
\hline RS3(108)-RS3(147) & 15 & 2 & 4 & 1 & & 6 & 2 & $1.7 \mathrm{E}-05$ & 13.9 \\
\hline RL1(141)-RL1(167) & 14 & 5 & 8 & 1 & & & & $6.3 \mathrm{E}-21$ & - \\
\hline RL19(63)-RL19(106) & 12 & & & 2 & 2 & 2 & 6 & $7.0 \mathrm{E}-06$ & 14.5 \\
\hline RS17(16)-RS17(43) & 12 & & 1 & 3 & 2 & 2 & 4 & $6.4 \mathrm{E}-07$ & 14.5 \\
\hline RS4(156)-RS4(177) & 11 & & 2 & 5 & & 2 & 2 & $1.1 \mathrm{E}-15$ & 11.3 \\
\hline RL27(62)-RL27(72) & 11 & 2 & & 2 & 1 & 4 & 2 & $5.2 \mathrm{E}-07$ & 19.4 \\
\hline RL10(37)-RL10(105) & 11 & 4 & 4 & & 3 & & & $2.3 \mathrm{E}-05$ & - \\
\hline RL29(60)-RL29(4) & 10 & 3 & 2 & & 2 & 1 & 2 & $2.4 \mathrm{E}-04$ & 13.7 \\
\hline $\operatorname{RS3}(27)-\operatorname{RS} 3(62)$ & 10 & 2 & 5 & & 2 & & 1 & $4.3 \mathrm{E}-12$ & 14.4 \\
\hline RL1(141)-RL1(198) & 10 & 5 & 5 & & & & & $1.6 \mathrm{E}-14$ & - \\
\hline RL25(34)-RL25(73) & 10 & 4 & 4 & 2 & & & & $2.0 \mathrm{E}-11$ & 15.2 \\
\hline RS4(151)-RS4(177) & 9 & & 1 & 2 & & 2 & 4 & $4.9 \mathrm{E}-06$ & 9.7 \\
\hline RL32(32)-RL32(53) & 9 & & & 4 & 5 & & & $8.1 \mathrm{E}-08$ & 10.8 \\
\hline RL1(197)-RL1(200) & 9 & 3 & 6 & & & & & $1.1 \mathrm{E}-12$ & - \\
\hline
\end{tabular}




\begin{tabular}{|c|c|c|c|c|c|c|c|c|c|}
\hline RS4(156)-RS4(167) & 9 & & 1 & 6 & 2 & & & $1.1 \mathrm{E}-19$ & 16.5 \\
\hline RL9(1)-RL9(22) & 8 & 1 & 2 & 5 & & & & $4.8 \mathrm{E}-22$ & 6.0 \\
\hline RS9(2)-RS9(13) & 7 & & 1 & & & 3 & 3 & $9.2 \mathrm{E}-05$ & - \\
\hline RL3(7)-RL3(56) & 7 & & & & & 3 & 4 & $5.7 \mathrm{E}-07$ & 17.6 \\
\hline RL4(1)-RL4(137) & 7 & 4 & 3 & & & & & $7.5 \mathrm{E}-07$ & 28.4 \\
\hline RS12(51)-RS12(108) & 7 & 2 & & & 5 & & & $1.5 \mathrm{E}-07$ & 15.6 \\
\hline RS13(103)-RS13(110) & 6 & & & 3 & 3 & & & $9.6 \mathrm{E}-06$ & 11.4 \\
\hline RL4(58)-RL4(63) & 6 & 2 & & 1 & 3 & & & $3.2 \mathrm{E}-05$ & 11.9 \\
\hline RS3(108)-RS3(219) & 6 & 2 & 3 & 1 & & & & $2.2 \mathrm{E}-15$ & - \\
\hline RS8(31)-RS8(41) & 6 & & & 3 & & 2 & 1 & $4.0 \mathrm{E}-06$ & 15.4 \\
\hline RS14(23)-RS14(47) & 6 & & 2 & 1 & & 1 & 2 & $1.2 \mathrm{E}-06$ & 16.0 \\
\hline RS21(54)-RS21(58) & 5 & 2 & 2 & & & & 1 & $1.3 \mathrm{E}-04$ & - \\
\hline RL4(6)-RL4(185) & 5 & 1 & & & 4 & & & $5.4 \mathrm{E}-15$ & 12.4 \\
\hline RS3(62)-RS3(89) & 5 & & & 1 & & 3 & 1 & $2.7 \mathrm{E}-07$ & 15.7 \\
\hline RS19(18)-RS19(28) & 5 & & 4 & & 1 & & & $7.6 \mathrm{E}-05$ & 11.0 \\
\hline RS18(9)-RS18(30) & 5 & & & 4 & & 1 & & $2.4 \mathrm{E}-07$ & - \\
\hline RL25(10)-RL25(25) & 4 & 3 & & & 1 & & & $1.1 \mathrm{E}-04$ & 13.5 \\
\hline RL4(132)-RL4(139) & 4 & 1 & 3 & & & & & $1.3 \mathrm{E}-10$ & 10.7 \\
\hline RL5(15)-RL5(120) & 4 & 1 & & 3 & & & & $3.4 \mathrm{E}-08$ & 21.2 \\
\hline RL1(31)-RL1(184) & 4 & & & 2 & 2 & & & $4.8 \mathrm{E}-07$ & - \\
\hline RS1(279)-RS1(347) & 4 & & & 3 & 1 & & & $2.0 \mathrm{E}-08$ & - \\
\hline RL3(105)-RL3(190) & 3 & & 1 & & 1 & & 1 & $1.8 \mathrm{E}-05$ & 10.3 \\
\hline RL4(1)-RL4(194) & 3 & 2 & 1 & & & & & $3.0 \mathrm{E}-05$ & 13.7 \\
\hline RS2(112)-RS2(115) & 3 & & 2 & & & & 1 & $2.8 \mathrm{E}-04$ & 5.0 \\
\hline RS20(33)-RS20(49) & 3 & & 1 & & & 1 & 1 & $3.6 \mathrm{E}-04$ & 12.8 \\
\hline RL17(35)-RL17(121) & 2 & & & 1 & 1 & & & $4.4 \mathrm{E}-04$ & - \\
\hline RS2(2)-RS2(11) & 2 & & 1 & & 1 & & & $1.6 \mathrm{E}-05$ & - \\
\hline RL4(1)-RL4(185) & 2 & & 1 & 1 & & & & $7.1 \mathrm{E}-05$ & 19.8 \\
\hline \multicolumn{10}{|c|}{ EDC } \\
\hline Site-pairs & \#total spec & \#Exp1 & \#Ехр2 & \#Ехр3 & \#Exp4 & \#Exp5 & \#Exp6 & Best E_value & $\begin{array}{c}\text { Ca-Ca } \\
\text { distance }\end{array}$ \\
\hline \multicolumn{10}{|l|}{ Inter Molecular } \\
\hline RS10(24)-RS3(219) & 59 & 5 & 3 & 15 & 9 & 14 & 13 & $5.9 E-18$ & - \\
\hline RL3(8)-RS7(67) & 31 & 7 & 4 & 4 & 5 & 4 & 7 & $2.9 \mathrm{E}-11$ & 174.8 \\
\hline RL5(15)-RL17(106) & 26 & 4 & 2 & 5 & 5 & 5 & 5 & $3.0 \mathrm{E}-13$ & 168.5 \\
\hline RS8(73)-RS5(156) & 22 & & 2 & 5 & 5 & 6 & 4 & $3.2 \mathrm{E}-08$ & 10.1 \\
\hline RL7(97)-RL11(113) & 20 & 6 & 3 & 5 & 5 & 1 & & $6.2 \mathrm{E}-18$ & - \\
\hline RS3(89)-RS2(241) & 16 & 1 & 1 & 2 & 4 & 4 & 4 & $2.8 \mathrm{E}-22$ & - \\
\hline RL28(10)-RL15(10) & 15 & & 4 & 3 & & 4 & 4 & $2.8 \mathrm{E}-07$ & 76.7 \\
\hline RS16(46)-DeaD(310) & 11 & 1 & 1 & 4 & 2 & 1 & 2 & 7.4E-10 & - \\
\hline RS3(89)-RL17(106) & 8 & & & 1 & & 4 & 3 & $2.6 \mathrm{E}-20$ & 178.8 \\
\hline RS3(89)-RL1(93) & 8 & & & 3 & & 2 & 3 & $7.6 \mathrm{E}-18$ & - \\
\hline RL7(85)-RL11(108) & 7 & 1 & & & 3 & & 3 & $2.2 \mathrm{E}-07$ & - \\
\hline RL9(86)-RL2(125) & 7 & & & & & 3 & 4 & $7.2 \mathrm{E}-10$ & 98.1 \\
\hline RS6(82)-RL9(89) & 7 & & & & & 4 & 3 & $6.6 \mathrm{E}-06$ & 85.4 \\
\hline RS5(156)-OmpA(331) & 7 & 1 & & 2 & 2 & & 2 & $6.1 \mathrm{E}-08$ & - \\
\hline YfiA(113)-RS7(149) & 6 & & & 1 & & 2 & 3 & $3.3 \mathrm{E}-20$ & - \\
\hline RL31(50)-RL5(179) & 6 & & & & & 2 & 4 & 7.7E-12 & - \\
\hline
\end{tabular}




\begin{tabular}{|c|c|c|c|c|c|c|c|c|c|}
\hline RS7(56)-RS2(188) & 5 & 2 & & 2 & & & 1 & 4.7E-16 & 90.5 \\
\hline RS7(17)-RS9(42) & 4 & & & 2 & & & 2 & $4.0 \mathrm{E}-04$ & 11.1 \\
\hline RS19(24)-RL31(70) & 4 & & & 2 & 2 & & & $2.4 \mathrm{E}-06$ & - \\
\hline RS9(89)-RS3(225) & 4 & & & 3 & 1 & & & 2.0E-11 & - \\
\hline RL7(85)-RL6(166) & 4 & & & 1 & & & 3 & $8.4 \mathrm{E}-07$ & - \\
\hline RL1(22)-NUOCD(200) & 3 & & 1 & 1 & & & 1 & $3.4 \mathrm{E}-05$ & - \\
\hline RL14(4)-RL29(9) & 3 & & & 1 & 1 & 1 & & $3.6 \mathrm{E}-08$ & 130.2 \\
\hline RS7(56)-RS2(175) & 3 & 1 & 1 & & 1 & & & $1.7 \mathrm{E}-12$ & 91.6 \\
\hline RL1(22)-RL17(121) & 3 & & & & & 1 & 2 & $1.3 \mathrm{E}-06$ & - \\
\hline RS10(27)-RS3(219) & 2 & & 1 & & & 1 & & $4.0 \mathrm{E}-12$ & - \\
\hline RL11(37)-PutA(1099) & 2 & & & 1 & & 1 & & $3.0 \mathrm{E}-06$ & - \\
\hline RS7(40)-RS2(59) & 2 & & & & & 1 & 1 & $1.6 \mathrm{E}-06$ & 97.7 \\
\hline RL7(96)-RPOB(937) & 2 & & 1 & & 1 & & & $1.1 \mathrm{E}-06$ & - \\
\hline \multicolumn{10}{|l|}{ Intra Molecular } \\
\hline RS1(527)-RS1(536) & 100 & 7 & 15 & 21 & 10 & 24 & 23 & $1.3 \mathrm{E}-11$ & - \\
\hline RL7(96)-RL7(102) & 74 & 15 & 11 & 18 & 18 & 5 & 7 & $6.6 \mathrm{E}-14$ & - \\
\hline RS8(50)-RS8(58) & 67 & 6 & 8 & 11 & 7 & 18 & 17 & $8.5 \mathrm{E}-16$ & 8.5 \\
\hline RL1(183)-RL1(186) & 60 & 4 & 6 & 9 & 11 & 16 & 14 & $7.2 \mathrm{E}-13$ & - \\
\hline RL23(26)-RL23(100) & 35 & 4 & 1 & 9 & 4 & 8 & 9 & $7.2 \mathrm{E}-14$ & - \\
\hline RL17(82)-RL17(121) & 29 & & 3 & 7 & 8 & 5 & 6 & $7.0 \mathrm{E}-15$ & - \\
\hline RL18(63)-RL18(69) & 19 & 4 & 1 & 4 & 4 & 3 & 3 & $6.2 \mathrm{E}-14$ & 8.4 \\
\hline RL7(71)-RL7(97) & 11 & 1 & & 2 & & 4 & 4 & $3.8 \mathrm{E}-08$ & - \\
\hline RS8(41)-RS8(52) & 9 & & 1 & 1 & & 2 & 5 & $3.7 \mathrm{E}-09$ & 16.6 \\
\hline RS1(264)-RS1(272) & 5 & 2 & & 1 & 1 & 1 & & $6.6 \mathrm{E}-08$ & - \\
\hline RL23(26)-RL23(94) & 4 & & & 1 & 3 & & & $6.5 \mathrm{E}-08$ & - \\
\hline RS9(1)-RS9(89) & 4 & & & & & 2 & 2 & $2.7 \mathrm{E}-10$ & - \\
\hline RL20(84)-RL20(89) & 4 & & & 1 & 1 & 1 & 1 & $8.1 \mathrm{E}-08$ & 5.8 \\
\hline RL9(83)-RL9(149) & 3 & & & & 1 & 1 & 1 & 4.7E-12 & 5.5 \\
\hline RL17(106)-RL17(121) & 2 & & & & 1 & 1 & & $4.5 \mathrm{E}-07$ & - \\
\hline RS7(40)-RS7(56) & 2 & 1 & & & 1 & & & $1.7 \mathrm{E}-12$ & 21.4 \\
\hline RS9(1)-RS9(3) & 2 & & & & & 1 & 1 & $3.3 \mathrm{E}-04$ & - \\
\hline RS8(50)-RS8(60) & 2 & & & 1 & & & 1 & $2.9 \mathrm{E}-06$ & 5.1 \\
\hline RL16(84)-RL16(90) & 2 & & & & & 1 & 1 & $9.3 \mathrm{E}-05$ & 17.2 \\
\hline
\end{tabular}


Supplemental Table 3. Cross-links identified between Cbf5 and Gar1.

\begin{tabular}{cccccccccc}
\hline Site pairs & $\begin{array}{c}\text { Total } \\
\text { Spec }\end{array}$ & BS $^{2} \mathrm{G}$ & BS $^{3}$ & DSS & EGS & GMBS & S-GMBS & $\begin{array}{c}\text { Best } \\
\text { E-value }\end{array}$ & $\begin{array}{c}\mathrm{C}_{\alpha}-\mathrm{C}_{\alpha} \\
\text { distance }^{{ }^{a}}\end{array}$ \\
\hline Cbf5(161)-Gar1(115) & 307 & 79 & 69 & 91 & 68 & & & $1.5 \mathrm{E}-14$ & 18.9 \\
Cbf5(161)-Gar1(77) & 85 & 16 & 24 & 26 & 19 & & & $8.2 \mathrm{E}-10$ & 18.9 \\
Cbf5(161)-Gar1(59) & 55 & & 19 & 36 & & & & $2.0 \mathrm{E}-07$ & 22.0 \\
Cbf5(134)-Gar1(104) & 29 & & & & 29 & & & $2.3 \mathrm{E}-15$ & 18.4 \\
Cbf5(190)-Gar1(59) & 28 & & & & & 9 & 29 & $4.3 \mathrm{E}-11$ & 24.9 \\
\hline
\end{tabular}

${ }^{\mathrm{a}}$ The $\mathrm{C}_{\alpha}-\mathrm{C}_{\alpha}$ distance is calculated based on the crystal structure of the CNG subcomplex (3U28). 
Supplemental Table 4. Cross-links identified between Nop10 and Nhp2.

\begin{tabular}{|c|c|c|c|c|c|c|c|c|}
\hline Site pairs & $\begin{array}{l}\text { Total } \\
\text { Spec }\end{array}$ & $\mathrm{BS}^{2} \mathrm{G}$ & $\mathrm{BS}^{3}$ & DSS & EGS & EDC & $\begin{array}{c}\text { Best } \\
\text { E-value }\end{array}$ & $\begin{array}{c}\mathrm{C}_{\alpha}-\mathrm{C}_{\alpha} \\
\text { distance }^{\mathrm{a}}\end{array}$ \\
\hline Nhp2(61)-Nop10(40) & 334 & 68 & 46 & 91 & 129 & & $3.8 \mathrm{E}-11$ & 12.3 \\
\hline Nhp2(65)-Nop10(40) & 180 & 27 & 20 & 46 & 87 & & $3.8 \mathrm{E}-16$ & 14.0 \\
\hline Nhp2(32)-Nop10(28) & 178 & & & & 178 & & $1.0 \mathrm{E}-17$ & 18.1 \\
\hline Nhp2(69)-Nop10(40) & 174 & 29 & 26 & 35 & 84 & & $7.3 \mathrm{E}-07$ & 17.2 \\
\hline Nhp2(107)-Nop10(19) & 41 & 26 & & 15 & & & $1.3 \mathrm{E}-07$ & 13.3 \\
\hline Nhp2(107)-Nop10(1) & 30 & & & 30 & & & $5.8 \mathrm{E}-14$ & 17.3 \\
\hline Nhp2(107)-Nop10(28) & 10 & & & 10 & & & $9.2 \mathrm{E}-06$ & 14.8 \\
\hline Nhp2(15)-Nop10(19) ${ }^{b}$ & 35 & 5 & & 20 & 10 & & $1.3 \mathrm{E}-16$ & - \\
\hline Nhp2(9)-Nop10(22) ${ }^{b}$ & 4 & & & & & 4 & $5.8 \mathrm{E}-06$ & - \\
\hline
\end{tabular}

Cross-links that are uniquely identified with one cross-linker are bolded.

${ }^{a}$ The $\mathrm{C}_{\alpha}-\mathrm{C}_{\alpha}$ distance is calculated based on the homology model of the CNGP complex.

${ }^{\mathrm{b}}$ They cannot be used for modeling because the first 26 amino acids are missing in the homology model of Nhp2. 
Supplemental Table 5. Clusters from docking of Gar1 onto Cbf5-Nop10 using Rosetta.

\begin{tabular}{|c|c|c|c|c|c|}
\hline \multirow[t]{2}{*}{ Cluster } & \multirow{2}{*}{$\begin{array}{c}\text { Energy } \\
\text { Rank }\end{array}$} & \multirow{2}{*}{$\begin{array}{c}\text { Cluster } \\
\text { size } \\
\end{array}$} & \multirow{2}{*}{ Energy } & \multicolumn{2}{|c|}{ RMSD to native } \\
\hline & & & & Low_res docking & Local refine \\
\hline \multicolumn{6}{|c|}{ No constraints } \\
\hline cluster 1 & 1 & 19 & -81.838 & 46.86 & 49.42 \\
\hline cluster2 & 17 & 17 & -68.562 & 35.57 & 39.42 \\
\hline cluster3 & 6 & 15 & -72.538 & 42.70 & 42.38 \\
\hline cluster 4 & 9 & 13 & -71.093 & 50.99 & 55.24 \\
\hline cluster5 & 14 & 12 & -69.170 & 57.75 & 59.38 \\
\hline cluster6 & 2 & 10 & -78.305 & 64.16 & 63.54 \\
\hline cluster7 & 10 & 10 & -70.541 & 44.30 & 44.95 \\
\hline cluster8 & 3 & 9 & -75.604 & 31.70 & 33.65 \\
\hline cluster9 & 19 & 9 & -67.314 & 46.70 & 47.31 \\
\hline cluster 10 & 15 & 8 & -68.741 & 47.14 & 43.33 \\
\hline cluster11 & 16 & 8 & -68.672 & 52.16 & 49.29 \\
\hline cluster12 & 7 & 7 & -71.968 & 33.65 & 33.24 \\
\hline cluster13 & 8 & 7 & -71.273 & 37.14 & 33.58 \\
\hline cluster14 & 4 & 6 & -75.386 & 19.89 & 20.03 \\
\hline cluster 15 & 18 & 5 & -68.021 & 75.77 & 79.63 \\
\hline cluster16 & 24 & 5 & -66.827 & 34.86 & 36.57 \\
\hline cluster17 & 27 & 5 & -65.631 & 49.68 & 51.26 \\
\hline cluster18 & 20 & 4 & -67.238 & 76.96 & 80.59 \\
\hline cluster19 & 25 & 4 & -66.446 & 38.35 & 39.53 \\
\hline cluster20 & 26 & 4 & -65.897 & 30.19 & 26.60 \\
\hline cluster21 & 5 & 3 & -75.018 & 38.95 & 36.07 \\
\hline cluster22 & 11 & 3 & -70.336 & 60.93 & 63.28 \\
\hline cluster23 & 22 & 3 & -67.195 & 60.71 & 65.26 \\
\hline cluster24 & 23 & 3 & -67.146 & 39.37 & 42.45 \\
\hline cluster25 & 28 & 3 & -64.803 & 74.11 & 82.17 \\
\hline cluster26 & 12 & 2 & -69.560 & 40.69 & 43.04 \\
\hline cluster27 & 13 & 2 & -69.214 & 18.75 & 26.23 \\
\hline cluster28 & 21 & 2 & -67.228 & 55.07 & 58.56 \\
\hline cluster29 & 29 & 2 & -62.170 & 70.01 & 73.23 \\
\hline cluster30 & 21 & 2 & -67.228 & 55.07 & 58.56 \\
\hline cluster31 & 13 & 2 & -69.214 & 18.75 & 26.23 \\
\hline cluster32 & 12 & 2 & -69.560 & 40.69 & 43.04 \\
\hline \multicolumn{6}{|c|}{ Three constraints from $\mathrm{BS}^{3}$ and DSS } \\
\hline cluster1 & 1 & 22 & -70.611 & 19.70 & 19.88 \\
\hline cluster2 & 6 & 20 & -66.699 & 44.53 & 44.96 \\
\hline cluster3 & 10 & 20 & -65.033 & 31.97 & 31.27 \\
\hline cluster4 & 15 & 17 & -62.672 & 23.73 & 25.15 \\
\hline cluster5 & 4 & 16 & -68.064 & 37.25 & 36.24 \\
\hline
\end{tabular}




\begin{tabular}{|c|c|c|c|c|c|}
\hline cluster6 & 11 & 16 & -64.725 & 41.25 & 43.44 \\
\hline cluster7 & 2 & 13 & -69.706 & 34.09 & 33.57 \\
\hline cluster8 & 7 & 13 & -65.841 & 41.62 & 45.83 \\
\hline cluster9 & 8 & 11 & -65.420 & 41.73 & 43.38 \\
\hline cluster10 & 5 & 8 & -67.020 & 34.19 & 36.71 \\
\hline cluster11 & 12 & 7 & -63.898 & 7.25 & 1.83 \\
\hline cluster12 & 13 & 7 & -63.681 & 41.32 & 43.89 \\
\hline cluster13 & 16 & 7 & -62.518 & 13.15 & 2.72 \\
\hline cluster14 & 14 & 6 & -63.424 & 35.43 & 32.93 \\
\hline cluster15 & 17 & 6 & -60.101 & 35.95 & 34.78 \\
\hline cluster16 & 9 & 5 & -65.120 & 40.15 & 36.26 \\
\hline cluster17 & 18 & 4 & -59.700 & 23.91 & 26.58 \\
\hline cluster18 & 3 & 2 & -69.296 & 41.17 & 38.82 \\
\hline \multicolumn{6}{|c|}{ Three constraints plus additional filter } \\
\hline cluster1 & 8 & 21 & -64.725 & 41.25 & 43.44 \\
\hline cluster2 & 7 & 20 & -65.033 & 31.97 & 31.27 \\
\hline cluster3 & 1 & 17 & -69.706 & 34.09 & 33.57 \\
\hline cluster 4 & 13 & 17 & -62.166 & 18.00 & 19.83 \\
\hline cluster5 & 4 & 15 & -66.699 & 44.53 & 44.96 \\
\hline cluster6 & 5 & 13 & -65.420 & 41.73 & 43.38 \\
\hline cluster7 & 11 & 12 & -63.424 & 35.43 & 32.93 \\
\hline cluster8 & 17 & 10 & -60.101 & 35.95 & 34.78 \\
\hline cluster9 & 2 & 9 & -69.350 & 19.46 & 20.14 \\
\hline cluster10 & 9 & 9 & -63.898 & 7.25 & 1.83 \\
\hline cluster11 & 14 & 9 & -61.542 & 26.14 & 32.65 \\
\hline cluster12 & 16 & 9 & -61.375 & 30.90 & 31.10 \\
\hline cluster13 & 6 & 8 & -65.120 & 40.15 & 36.26 \\
\hline cluster14 & 12 & 8 & -63.151 & 39.26 & 43.35 \\
\hline cluster15 & 10 & 7 & -63.681 & 41.32 & 42.44 \\
\hline cluster16 & 15 & 6 & -61.486 & 33.74 & 31.78 \\
\hline cluster17 & 3 & 5 & -67.020 & 34.19 & 36.71 \\
\hline cluster18 & 18 & 5 & -57.373 & 39.25 & 40.01 \\
\hline \multicolumn{6}{|c|}{ Four constraints (with $\mathrm{BS}^{3}$, DSS and Sulfo-GMBS) } \\
\hline cluster1 & 2 & 25 & -69.074 & 47.85 & 45.64 \\
\hline cluster2 & 3 & 24 & -68.772 & 36.86 & 37.30 \\
\hline cluster3 & 10 & 19 & -64.087 & 41.57 & 42.86 \\
\hline cluster 4 & 1 & 17 & -69.814 & 32.37 & 39.56 \\
\hline cluster5 & 5 & 17 & -67.023 & 36.40 & 30.95 \\
\hline cluster6 & 7 & 13 & -65.563 & 20.97 & 19.71 \\
\hline cluster7 & 9 & 11 & -64.370 & 33.49 & 28.54 \\
\hline cluster8 & 12 & 10 & -63.049 & 19.08 & 25.59 \\
\hline cluster9 & 6 & 9 & -66.074 & 36.15 & 33.30 \\
\hline cluster10 & 15 & 8 & -62.783 & 37.27 & 33.80 \\
\hline
\end{tabular}




\begin{tabular}{|c|c|c|c|c|c|}
\hline cluster11 & 17 & 8 & -61.083 & 29.72 & 27.19 \\
\hline cluster12 & 4 & 7 & -68.591 & 8.59 & 2.27 \\
\hline cluster13 & 8 & 7 & -64.624 & 30.77 & 39.58 \\
\hline cluster14 & 16 & 7 & -62.167 & 40.50 & 34.24 \\
\hline cluster15 & 11 & 5 & -63.567 & 25.99 & 26.82 \\
\hline cluster16 & 18 & 3 & -59.336 & 39.96 & 42.90 \\
\hline cluster17 & 19 & 3 & -59.027 & 25.82 & 26.66 \\
\hline cluster18 & 20 & 3 & -57.422 & 18.96 & 17.56 \\
\hline cluster19 & 13 & 2 & -62.892 & 40.68 & 46.64 \\
\hline cluster20 & 14 & 2 & -62.788 & 43.59 & 40.83 \\
\hline \multicolumn{6}{|c|}{ Four constraints (with $\mathrm{BS}^{3}$, DSS and Sulfo-GMBS) plus additional filter } \\
\hline cluster1 & 9 & 23 & -64.113 & 39.61 & 38.43 \\
\hline cluster 2 & 1 & 20 & -69.814 & 32.37 & 39.56 \\
\hline cluster3 & 2 & 19 & -69.074 & 47.85 & 45.64 \\
\hline cluster 4 & 4 & 15 & -68.591 & 8.59 & 2.27 \\
\hline cluster 5 & 7 & 15 & -64.981 & 31.28 & 23.12 \\
\hline cluster6 & 8 & 14 & -64.441 & 19.98 & 20.11 \\
\hline cluster7 & 6 & 10 & -66.074 & 36.15 & 34.74 \\
\hline cluster8 & 12 & 10 & -63.049 & 19.08 & 24.86 \\
\hline cluster9 & 5 & 9 & -68.490 & 35.23 & 39.91 \\
\hline cluster10 & 11 & 9 & -63.509 & 23.43 & 25.11 \\
\hline cluster11 & 16 & 9 & -59.756 & 47.82 & 47.61 \\
\hline cluster12 & 19 & 9 & -58.445 & 51.72 & 51.39 \\
\hline cluster13 & 14 & 8 & -62.167 & 40.50 & 32.44 \\
\hline cluster14 & 3 & 7 & -68.772 & 36.86 & 37.30 \\
\hline cluster15 & 10 & 6 & -63.964 & 32.31 & 26.97 \\
\hline cluster16 & 13 & 6 & -62.892 & 40.68 & 46.64 \\
\hline cluster17 & 15 & 5 & -60.484 & 30.84 & 26.27 \\
\hline cluster18 & 17 & 3 & -59.603 & 24.56 & 20.53 \\
\hline cluster19 & 18 & 3 & -59.027 & 25.82 & 26.66 \\
\hline \multicolumn{6}{|c|}{ Four constraints (with $\mathrm{BS}^{3}$, DSS and EGS ) } \\
\hline cluster1 & 5 & 27 & -63.657 & 30.86 & 25.77 \\
\hline cluster2 & 2 & 21 & -65.797 & 31.54 & 28.15 \\
\hline cluster3 & 6 & 21 & -62.269 & 11.05 & 10.24 \\
\hline cluster4 & 7 & 21 & -61.910 & 26.58 & 30.28 \\
\hline cluster5 & 3 & 17 & -64.501 & 19.76 & 20.21 \\
\hline cluster6 & 9 & 13 & -61.114 & 12.73 & 5.07 \\
\hline cluster7 & 11 & 13 & -59.926 & 34.34 & 34.36 \\
\hline cluster8 & 8 & 12 & -61.394 & 25.11 & 25.68 \\
\hline cluster9 & 13 & 12 & -58.029 & 25.55 & 30.38 \\
\hline cluster10 & 4 & 11 & -63.691 & 37.76 & 35.40 \\
\hline cluster11 & 10 & 10 & -60.795 & 5.39 & 2.23 \\
\hline cluster12 & 14 & 8 & -56.610 & 28.96 & 29.78 \\
\hline
\end{tabular}




\begin{tabular}{|c|c|c|c|c|c|}
\hline cluster13 & 1 & 6 & -66.661 & 33.88 & 38.62 \\
\hline cluster14 & 12 & 6 & -59.777 & 33.77 & 34.57 \\
\hline cluster 15 & 15 & 2 & -56.248 & 41.23 & 43.06 \\
\hline \multicolumn{6}{|c|}{ Four constraints(with $\mathrm{BS}^{3}$, DSS and EGS) plus additional filter } \\
\hline cluster1 & 3 & 33 & -62.269 & 11.05 & 10.24 \\
\hline cluster2 & 5 & 30 & -60.847 & 31.61 & 33.91 \\
\hline cluster3 & 2 & 26 & -63.657 & 30.86 & 25.77 \\
\hline cluster4 & 4 & 25 & -61.910 & 26.58 & 30.28 \\
\hline cluster5 & 6 & 19 & -60.795 & 5.39 & 2.23 \\
\hline cluster6 & 7 & 18 & -60.198 & 12.93 & 2.70 \\
\hline cluster7 & 10 & 15 & -54.773 & 19.02 & 25.61 \\
\hline cluster8 & 8 & 14 & -58.092 & 15.74 & 15.20 \\
\hline cluster9 & 1 & 9 & -63.691 & 37.76 & 35.40 \\
\hline cluster10 & 9 & 8 & -55.036 & 18.25 & 20.12 \\
\hline cluster11 & 11 & 3 & -53.147 & 25.53 & 28.54 \\
\hline \multicolumn{6}{|c|}{ All five constraints } \\
\hline cluster1 & 4 & 27 & -64.650 & 32.74 & 40.81 \\
\hline cluster2 & 8 & 22 & -62.307 & 6.83 & 2.54 \\
\hline cluster3 & 6 & 20 & -63.069 & 33.85 & 29.17 \\
\hline cluster4 & 13 & 19 & -58.906 & 17.31 & 19.23 \\
\hline cluster5 & 11 & 17 & -59.459 & 22.05 & 23.37 \\
\hline cluster6 & 7 & 15 & -62.939 & 34.51 & 33.71 \\
\hline cluster7 & 9 & 14 & -61.629 & 7.52 & 2.58 \\
\hline cluster8 & 15 & 14 & -56.249 & 17.70 & 24.77 \\
\hline cluster9 & 1 & 13 & -71.174 & 32.67 & 27.79 \\
\hline cluster10 & 10 & 13 & -61.458 & 41.07 & 45.40 \\
\hline cluster11 & 12 & 13 & -59.078 & 8.47 & 2.09 \\
\hline cluster12 & 3 & 5 & -64.679 & 35.76 & 37.53 \\
\hline cluster13 & 2 & 3 & -66.084 & 34.00 & 39.43 \\
\hline cluster14 & 14 & 3 & -56.280 & 36.31 & 37.36 \\
\hline cluster 15 & 5 & 2 & -64.383 & 37.12 & 45.71 \\
\hline \multicolumn{6}{|c|}{ All five constraints plus additional filter } \\
\hline cluster1 & 4 & 26 & -60.327 & 4.63 & 2.34 \\
\hline cluster2 & 1 & 25 & -64.650 & 32.74 & 40.81 \\
\hline cluster3 & 2 & 23 & -62.307 & 6.83 & 2.54 \\
\hline cluster4 & 3 & 23 & -61.629 & 7.52 & 2.58 \\
\hline cluster5 & 7 & 21 & -58.906 & 17.31 & 19.23 \\
\hline cluster6 & 10 & 18 & -54.061 & 9.98 & 1.98 \\
\hline cluster7 & 5 & 14 & -59.459 & 22.05 & 23.37 \\
\hline cluster8 & 8 & 13 & -57.817 & 29.56 & 29.52 \\
\hline cluster9 & 9 & 13 & -54.859 & 18.52 & 25.34 \\
\hline cluster10 & 6 & 11 & -59.370 & 21.13 & 21.18 \\
\hline cluster11 & 11 & 11 & -53.804 & 12.35 & 20.11 \\
\hline
\end{tabular}


cluster12 
Supplemental Table 6. Clusters from docking of Nhp2onto Cbf5-Nop10-Gar1 using Rosetta.

\begin{tabular}{|c|c|c|c|c|c|}
\hline \multirow{2}{*}{ Cluster } & \multirow{2}{*}{ Energy Rank } & \multirow{2}{*}{$\begin{array}{c}\text { Cluster } \\
\text { size }\end{array}$} & \multirow{2}{*}{ Energy } & \multicolumn{2}{|c|}{ RMSD to homology model } \\
\hline & & & & Low_res docking & Local refine \\
\hline \multicolumn{6}{|c|}{ No constraints } \\
\hline cluster1 & 8 & 7 & -100.446 & 58.37 & 64.15 \\
\hline cluster2 & 14 & 7 & -96.585 & 64.04 & 67.98 \\
\hline cluster3 & 11 & 6 & -96.887 & 60.44 & 66.42 \\
\hline cluster4 & 13 & 6 & -96.833 & 62.11 & 61.84 \\
\hline cluster5 & 17 & 6 & -96.040 & 67.14 & 78.42 \\
\hline cluster6 & 2 & 5 & -103.473 & 45.47 & 40.53 \\
\hline cluster7 & 3 & 5 & -102.932 & 62.84 & 64.14 \\
\hline cluster8 & 19 & 5 & -95.343 & 49.19 & 50.87 \\
\hline cluster9 & 29 & 5 & -93.068 & 58.09 & 60.87 \\
\hline cluster 10 & 33 & 5 & -92.299 & 46.55 & 45.98 \\
\hline cluster11 & 4 & 4 & -101.915 & 61.47 & 62.19 \\
\hline cluster12 & 15 & 4 & -96.092 & 55.31 & 55.31 \\
\hline cluster13 & 23 & 4 & -94.116 & 51.40 & 48.19 \\
\hline cluster14 & 25 & 4 & -93.640 & 29.78 & 25.33 \\
\hline cluster 15 & 28 & 4 & -93.119 & 47.34 & 50.81 \\
\hline cluster16 & 30 & 4 & -92.686 & 36.41 & 37.57 \\
\hline cluster17 & 32 & 4 & -92.540 & 42.80 & 48.22 \\
\hline cluster 18 & 35 & 4 & -91.979 & 59.90 & 59.45 \\
\hline cluster19 & 39 & 4 & -91.629 & 68.11 & 74.02 \\
\hline cluster20 & 46 & 4 & -90.819 & 47.33 & 46.70 \\
\hline cluster 21 & 50 & 4 & -90.168 & 73.51 & 76.09 \\
\hline cluster22 & 52 & 4 & -90.145 & 42.74 & 47.79 \\
\hline cluster 23 & 1 & 3 & -107.388 & 57.83 & 55.78 \\
\hline cluster24 & 5 & 3 & -101.616 & 58.22 & 54.47 \\
\hline cluster 25 & 7 & 3 & -101.345 & 67.76 & 72.45 \\
\hline cluster26 & 18 & 3 & -96.030 & 56.43 & 58.28 \\
\hline cluster 27 & 22 & 3 & -94.273 & 47.35 & 52.45 \\
\hline cluster28 & 26 & 3 & -93.461 & 74.25 & 75.13 \\
\hline cluster29 & 31 & 3 & -92.669 & 61.78 & 73.01 \\
\hline cluster30 & 36 & 3 & -91.937 & 35.77 & 39.03 \\
\hline cluster31 & 37 & 3 & -91.838 & 23.44 & 22.33 \\
\hline cluster32 & 42 & 3 & -91.391 & 66.32 & 74.00 \\
\hline cluster33 & 66 & 3 & -88.920 & 63.42 & 67.68 \\
\hline cluster34 & 9 & 2 & -97.695 & 35.81 & 40.27 \\
\hline cluster 35 & 10 & 2 & -97.481 & 36.17 & 42.30 \\
\hline cluster36 & 12 & 2 & -96.835 & 39.10 & 36.81 \\
\hline
\end{tabular}




\begin{tabular}{|c|c|c|c|c|c|}
\hline cluster37 & 16 & 2 & -96.062 & 49.71 & 48.87 \\
\hline cluster38 & 20 & 2 & -95.031 & 61.48 & 61.41 \\
\hline cluster39 & 21 & 2 & -94.383 & 15.08 & 11.70 \\
\hline cluster 40 & 24 & 2 & -93.956 & 15.40 & 14.66 \\
\hline cluster41 & 27 & 2 & -93.345 & 36.13 & 41.65 \\
\hline cluster42 & 34 & 2 & -92.108 & 72.94 & 72.88 \\
\hline cluster 43 & 38 & 2 & -91.762 & 71.03 & 79.20 \\
\hline cluster44 & 40 & 2 & -91.494 & 63.66 & 65.75 \\
\hline cluster45 & 41 & 2 & -91.411 & 66.27 & 65.80 \\
\hline cluster46 & 43 & 2 & -91.221 & 42.44 & 44.91 \\
\hline cluster47 & 45 & 2 & -90.960 & 18.65 & 22.54 \\
\hline cluster 48 & 47 & 2 & -90.789 & 70.68 & 73.76 \\
\hline cluster49 & 48 & 2 & -90.500 & 72.86 & 82.53 \\
\hline cluster50 & 49 & 2 & -90.314 & 54.50 & 59.68 \\
\hline cluster51 & 54 & 2 & -90.037 & 23.44 & 24.27 \\
\hline cluster52 & 57 & 2 & -89.858 & 59.19 & 61.15 \\
\hline cluster53 & 59 & 2 & -89.595 & 62.09 & 71.01 \\
\hline cluster54 & 61 & 2 & -89.555 & 31.20 & 29.99 \\
\hline cluster55 & 65 & 2 & -89.022 & 56.82 & 62.41 \\
\hline cluster56 & 67 & 2 & -88.519 & 57.17 & 58.54 \\
\hline cluster57 & 69 & 2 & -88.314 & 84.94 & 88.86 \\
\hline cluster 58 & 6 & 1 & -101.542 & 41.19 & 42.16 \\
\hline cluster59 & 44 & 1 & -91.008 & 25.79 & 24.56 \\
\hline cluster60 & 51 & 1 & -90.151 & 61.21 & 59.99 \\
\hline cluster61 & 53 & 1 & -90.082 & 69.10 & 71.71 \\
\hline cluster62 & 55 & 1 & -90.026 & 58.09 & 62.50 \\
\hline cluster63 & 56 & 1 & -89.880 & 58.80 & 63.37 \\
\hline cluster64 & 58 & 1 & -89.751 & 76.20 & 82.50 \\
\hline cluster65 & 60 & 1 & -89.587 & 56.11 & 63.07 \\
\hline cluster66 & 62 & 1 & -89.176 & 62.10 & 58.76 \\
\hline cluster67 & 63 & 1 & -89.058 & 43.36 & 48.79 \\
\hline cluster68 & 64 & 1 & -89.029 & 43.91 & 42.89 \\
\hline cluster69 & 68 & 1 & -88.349 & 51.85 & 68.95 \\
\hline cluster70 & 70 & 1 & -88.063 & 37.65 & 46.17 \\
\hline cluster71 & 71 & 1 & -88.032 & 55.92 & 65.08 \\
\hline \multicolumn{6}{|c|}{ No constraints with additional filter } \\
\hline cluster1 & 1 & 18 & -94.383 & 15.08 & 15.47 \\
\hline cluster2 & 13 & 14 & -84.456 & 21.38 & 21.16 \\
\hline cluster3 & 5 & 12 & -90.96 & 18.65 & 31.4 \\
\hline cluster4 & 14 & 12 & -84.042 & 16.03 & 18.76 \\
\hline cluster5 & 3 & 11 & -93.309 & 38.15 & 42 \\
\hline
\end{tabular}




\begin{tabular}{|c|c|c|c|c|c|}
\hline cluster6 & 20 & 10 & -80.127 & 11.06 & 13.79 \\
\hline cluster7 & 21 & 9 & -79.954 & 14.35 & 16 \\
\hline cluster8 & 8 & 9 & -87.332 & 16.03 & 15.32 \\
\hline cluster9 & 6 & 8 & -89.562 & 9.68 & 6.49 \\
\hline cluster10 & 7 & 8 & -89.36 & 24.77 & 16.69 \\
\hline cluster11 & 11 & 8 & -85.918 & 21.68 & 22.08 \\
\hline cluster12 & 9 & 7 & -87.32 & 10.07 & 11.6 \\
\hline cluster13 & 28 & 7 & -77.06 & 11.4 & 13.75 \\
\hline cluster14 & 2 & 7 & -93.956 & 15.4 & 17.06 \\
\hline cluster 15 & 12 & 7 & -84.547 & 26.29 & 31.42 \\
\hline cluster16 & 19 & 5 & -80.328 & 24.81 & 27.1 \\
\hline cluster17 & 25 & 5 & -78.169 & 26.27 & 25.68 \\
\hline cluster18 & 26 & 5 & -78.136 & 20.65 & 22.1 \\
\hline cluster19 & 22 & 4 & -79.569 & 17.06 & 14.92 \\
\hline cluster20 & 10 & 4 & -86.828 & 19.78 & 20.25 \\
\hline cluster21 & 4 & 4 & -91.008 & 25.79 & 26.84 \\
\hline cluster22 & 16 & 4 & -81.75 & 26.48 & 20.47 \\
\hline cluster23 & 17 & 3 & -81.456 & 18.97 & 18.46 \\
\hline cluster24 & 23 & 3 & -79.417 & 29.94 & 13.33 \\
\hline cluster 25 & 24 & 3 & -79.293 & 26.13 & 29.69 \\
\hline cluster26 & 15 & 3 & -81.811 & 29.86 & 34.79 \\
\hline cluster 27 & 27 & 3 & -77.93 & 26.85 & 23.75 \\
\hline cluster28 & 29 & 3 & -76.849 & 19.3 & 23.4 \\
\hline cluster29 & 18 & 2 & -80.607 & 29.1 & 20.35 \\
\hline cluster30 & 30 & 2 & -76.061 & 25.82 & 25 \\
\hline \multicolumn{6}{|c|}{ DSS constraints } \\
\hline cluster1 & 17 & 12 & -83.964 & 39.9 & 38.35 \\
\hline cluster2 & 9 & 10 & -86.681 & 17.88 & 18.84 \\
\hline cluster3 & 5 & 10 & -87.941 & 19.49 & 27.13 \\
\hline cluster 4 & 1 & 10 & -90.889 & 25.93 & 22.02 \\
\hline cluster5 & 23 & 8 & -82.254 & 21.28 & 23.69 \\
\hline cluster6 & 22 & 8 & -82.764 & 48.78 & 49.34 \\
\hline cluster7 & 10 & 8 & -86.476 & 27.77 & 36.67 \\
\hline cluster8 & 37 & 6 & -80.091 & 22.59 & 27.93 \\
\hline cluster9 & 2 & 6 & -88.691 & 39.7 & 46.63 \\
\hline cluster 10 & 15 & 6 & -84.945 & 40.33 & 35.32 \\
\hline cluster 11 & 13 & 6 & -85.609 & 47.31 & 50.49 \\
\hline cluster12 & 8 & 5 & -87.161 & 36.95 & 40.27 \\
\hline cluster13 & 7 & 5 & -87.273 & 22.32 & 23.35 \\
\hline cluster14 & 35 & 5 & -80.383 & 22.44 & 23.59 \\
\hline cluster15 & 34 & 5 & -80.688 & 28.01 & 27.06 \\
\hline
\end{tabular}




\begin{tabular}{|c|c|c|c|c|c|}
\hline cluster16 & 32 & 5 & -81.181 & 25.94 & 24.95 \\
\hline cluster17 & 29 & 5 & -81.568 & 21.36 & 22.75 \\
\hline cluster 18 & 18 & 5 & -83.472 & 23.48 & 23.25 \\
\hline cluster19 & 11 & 5 & -86.124 & 22.5 & 17.83 \\
\hline cluster20 & 39 & 4 & -79.907 & 21.49 & 24.34 \\
\hline cluster21 & 3 & 4 & -88.595 & 25.35 & 19.79 \\
\hline cluster22 & 31 & 4 & -81.35 & 30.15 & 34.08 \\
\hline cluster 23 & 25 & 4 & -82 & 30.21 & 31.47 \\
\hline cluster24 & 16 & 4 & -84.754 & 31 & 37.48 \\
\hline cluster 25 & 14 & 4 & -85.343 & 18.31 & 28.02 \\
\hline cluster26 & 6 & 3 & -87.356 & 22.91 & 26.13 \\
\hline cluster27 & 4 & 3 & -88.489 & 27.89 & 27.85 \\
\hline cluster28 & 38 & 3 & -79.956 & 16.47 & 21.49 \\
\hline cluster29 & 36 & 3 & -80.289 & 20.68 & 21.33 \\
\hline cluster30 & 27 & 3 & -81.776 & 31.73 & 29.04 \\
\hline cluster31 & 24 & 3 & -82.248 & 23.6 & 24.78 \\
\hline cluster32 & 21 & 3 & -82.891 & 12.95 & 12.65 \\
\hline cluster33 & 20 & 3 & -83.006 & 33.41 & 42.08 \\
\hline cluster34 & 19 & 3 & -83.375 & 26.4 & 23.08 \\
\hline cluster35 & 12 & 3 & -85.87 & 39.37 & 42.32 \\
\hline cluster36 & 43 & 2 & -78.466 & 26.37 & 25.54 \\
\hline cluster37 & 42 & 2 & -78.71 & 33.16 & 38.11 \\
\hline cluster38 & 40 & 2 & -79.834 & 32.98 & 33.59 \\
\hline cluster39 & 33 & 2 & -80.738 & 29.5 & 33.93 \\
\hline cluster40 & 30 & 2 & -81.543 & 40.83 & 38.32 \\
\hline cluster41 & 28 & 2 & -81.735 & 51.38 & 55.21 \\
\hline cluster42 & 26 & 2 & -81.813 & 18.05 & 15.63 \\
\hline cluster43 & 44 & 1 & -78.199 & 20.66 & 17.64 \\
\hline cluster44 & 41 & 1 & -78.778 & 40.85 & 46.33 \\
\hline \multicolumn{6}{|c|}{ DSS constraints with additional filter } \\
\hline cluster1 & 18 & 10 & -80.555 & 25.36 & 27.27 \\
\hline cluster2 & 8 & 9 & -84.754 & 31 & 35.21 \\
\hline cluster3 & 29 & 9 & -77.413 & 18.63 & 15.9 \\
\hline cluster4 & 5 & 8 & -86.681 & 17.88 & 25.63 \\
\hline cluster5 & 3 & 8 & -87.941 & 19.49 & 17.8 \\
\hline cluster6 & 24 & 8 & -78.768 & 17.14 & 15.13 \\
\hline cluster7 & 23 & 8 & -79.11 & 16.08 & 15.98 \\
\hline cluster8 & 22 & 8 & -79.121 & 19.65 & 17.96 \\
\hline cluster9 & 14 & 8 & -81.568 & 21.36 & 34.34 \\
\hline cluster10 & 30 & 7 & -77.354 & 27.99 & 26.76 \\
\hline cluster11 & 13 & 7 & -82.791 & 20.47 & 15.54 \\
\hline
\end{tabular}




\begin{tabular}{|c|c|c|c|c|c|}
\hline cluster12 & 6 & 6 & -86.476 & 27.77 & 29.63 \\
\hline cluster13 & 4 & 6 & -87.273 & 22.32 & 23.41 \\
\hline cluster14 & 27 & 6 & -78.199 & 20.66 & 25.95 \\
\hline cluster 15 & 1 & 6 & -88.595 & 25.35 & 19.2 \\
\hline cluster16 & 7 & 5 & -85.343 & 18.31 & 18.33 \\
\hline cluster17 & 32 & 5 & -77.154 & 21.26 & 30.33 \\
\hline cluster18 & 28 & 5 & -77.675 & 36.53 & 27.51 \\
\hline cluster19 & 21 & 5 & -79.357 & 22.11 & 32.14 \\
\hline cluster20 & 15 & 5 & -80.985 & 42.18 & 52.26 \\
\hline cluster 21 & 10 & 5 & -83.964 & 39.9 & 37.22 \\
\hline cluster22 & 9 & 4 & -84.17 & 26.14 & 27.47 \\
\hline cluster 23 & 37 & 4 & -74.398 & 19.53 & 22.32 \\
\hline cluster24 & 36 & 4 & -74.857 & 8.14 & 13.31 \\
\hline cluster 25 & 35 & 4 & -75.142 & 39.75 & 47.31 \\
\hline cluster26 & 33 & 4 & -76.927 & 19.52 & 18.79 \\
\hline cluster27 & 20 & 4 & -79.691 & 22.33 & 21.97 \\
\hline cluster 28 & 12 & 4 & -82.891 & 12.95 & 14.24 \\
\hline cluster29 & 11 & 4 & -83.341 & 35.63 & 32.82 \\
\hline cluster30 & 40 & 3 & -74.198 & 26.26 & 30.89 \\
\hline cluster31 & 39 & 3 & -74.233 & 26.16 & 29.17 \\
\hline cluster32 & 26 & 3 & -78.207 & 22.78 & 34.42 \\
\hline cluster33 & 17 & 3 & -80.799 & 28.03 & 26.16 \\
\hline cluster34 & 38 & 2 & -74.359 & 36.79 & 45.36 \\
\hline cluster35 & 31 & 2 & -77.192 & 21.64 & 22.78 \\
\hline cluster36 & 25 & 2 & -78.368 & 24.8 & 26.6 \\
\hline cluster37 & 2 & 2 & -88.489 & 27.89 & 49.11 \\
\hline cluster38 & 41 & 1 & -73.974 & 49.07 & 53.46 \\
\hline cluster39 & 34 & 1 & -75.661 & 20.68 & 15.22 \\
\hline cluster 40 & 19 & 1 & -79.834 & 32.98 & 30.65 \\
\hline cluster41 & 16 & 1 & -80.87 & 24.71 & 22.29 \\
\hline \multicolumn{6}{|c|}{ Seven constraints } \\
\hline cluster1 & 7 & 14 & -88.148 & 31.16 & 37.13 \\
\hline cluster2 & 30 & 10 & -83.545 & 19.34 & 18.39 \\
\hline cluster3 & 16 & 9 & -86.142 & 19.69 & 20.60 \\
\hline cluster4 & 3 & 8 & -90.854 & 36.92 & 40.61 \\
\hline cluster5 & 11 & 8 & -87.173 & 22.84 & 24.43 \\
\hline cluster6 & 26 & 8 & -84.102 & 22.86 & 21.30 \\
\hline cluster7 & 14 & 7 & -86.741 & 20.76 & 23.41 \\
\hline cluster8 & 19 & 7 & -85.420 & 21.43 & 20.37 \\
\hline cluster9 & 22 & 7 & -84.779 & 34.86 & 45.50 \\
\hline cluster10 & 6 & 6 & -88.969 & 22.46 & 21.36 \\
\hline
\end{tabular}




\begin{tabular}{|c|c|c|c|c|c|}
\hline cluster11 & 8 & 6 & -88.097 & 21.59 & 27.94 \\
\hline cluster12 & 17 & 6 & -86.062 & 20.36 & 21.56 \\
\hline cluster13 & 20 & 6 & -85.190 & 22.03 & 21.55 \\
\hline cluster14 & 37 & 6 & -81.661 & 30.84 & 32.52 \\
\hline cluster 15 & 1 & 5 & -97.022 & 34.28 & 32.16 \\
\hline cluster16 & 5 & 5 & -89.391 & 25.77 & 24.26 \\
\hline cluster17 & 13 & 5 & -87.011 & 30.88 & 45.87 \\
\hline cluster18 & 21 & 5 & -84.812 & 30.73 & 39.82 \\
\hline cluster19 & 23 & 5 & -84.607 & 24.61 & 24.39 \\
\hline cluster20 & 25 & 5 & -84.463 & 16.29 & 17.01 \\
\hline cluster21 & 27 & 5 & -84.098 & 39.64 & 45.92 \\
\hline cluster22 & 36 & 5 & -81.749 & 27.84 & 30.42 \\
\hline cluster23 & 2 & 4 & -91.826 & 31.39 & 29.80 \\
\hline cluster24 & 4 & 4 & -90.361 & 12.06 & 14.96 \\
\hline cluster 25 & 9 & 4 & -88.059 & 15.72 & 15.69 \\
\hline cluster26 & 10 & 4 & -87.701 & 21.30 & 23.08 \\
\hline cluster27 & 18 & 4 & -85.460 & 40.64 & 37.57 \\
\hline cluster28 & 35 & 4 & -81.880 & 25.99 & 19.43 \\
\hline cluster29 & 31 & 3 & -82.984 & 24.97 & 24.24 \\
\hline cluster30 & 32 & 3 & -82.924 & 41.02 & 39.61 \\
\hline cluster31 & 33 & 3 & -82.582 & 22.04 & 19.29 \\
\hline cluster32 & 34 & 3 & -82.487 & 22.28 & 22.28 \\
\hline cluster33 & 38 & 3 & -81.423 & 28.60 & 27.81 \\
\hline cluster34 & 12 & 2 & -87.115 & 23.68 & 26.80 \\
\hline cluster35 & 15 & 2 & -86.598 & 27.44 & 22.83 \\
\hline cluster36 & 24 & 2 & -84.508 & 24.26 & 23.78 \\
\hline cluster37 & 29 & 2 & -83.664 & 25.29 & 21.62 \\
\hline cluster38 & 40 & 2 & -79.845 & 33.92 & 40.99 \\
\hline cluster39 & 28 & 1 & -83.798 & 25.41 & 23.58 \\
\hline cluster40 & 39 & 1 & -79.904 & 34.48 & 37.06 \\
\hline cluster41 & 41 & 1 & -78.805 & 21.94 & 35.25 \\
\hline \multicolumn{6}{|c|}{ Seven constraints with additional filter } \\
\hline cluster1 & 12 & 13 & -84.472 & 19.67 & 24.59 \\
\hline cluster2 & 3 & 12 & -88.059 & 15.72 & 16.74 \\
\hline cluster3 & 8 & 12 & -86.062 & 20.36 & 19.43 \\
\hline cluster 4 & 2 & 11 & -88.097 & 21.59 & 32.91 \\
\hline cluster5 & 13 & 10 & -84.463 & 16.29 & 15.16 \\
\hline cluster6 & 1 & 9 & -90.361 & 12.06 & 10.62 \\
\hline cluster7 & 6 & 9 & -86.741 & 20.76 & 24.50 \\
\hline cluster8 & 7 & 9 & -86.142 & 19.69 & 20.92 \\
\hline cluster9 & 10 & 9 & -84.655 & 26.96 & 30.29 \\
\hline
\end{tabular}




\begin{tabular}{llllll} 
cluster10 & 16 & 8 & -83.444 & 21.30 & 21.94 \\
cluster11 & 19 & 8 & -82.023 & 9.75 & 10.30 \\
cluster12 & 21 & 8 & -81.880 & 25.99 & 20.50 \\
cluster13 & 14 & 7 & -83.907 & 19.02 & 20.56 \\
cluster14 & 25 & 7 & -80.283 & 19.31 & 22.37 \\
cluster15 & 4 & 6 & -87.701 & 21.30 & 15.26 \\
cluster16 & 11 & 6 & -84.551 & 25.65 & 29.24 \\
cluster17 & 29 & 6 & -77.420 & 14.79 & 16.54 \\
cluster18 & 17 & 5 & -83.358 & 22.33 & 19.22 \\
cluster19 & 18 & 5 & -82.487 & 22.28 & 22.29 \\
cluster20 & 24 & 5 & -80.764 & 19.29 & 19.07 \\
cluster21 & 28 & 5 & -77.587 & 25.26 & 24.52 \\
cluster22 & 15 & 4 & -83.471 & 34.76 & 35.37 \\
cluster23 & 22 & 4 & -81.166 & 26.72 & 30.70 \\
cluster24 & 5 & 3 & -87.115 & 23.68 & 25.11 \\
cluster25 & 9 & 3 & -84.812 & 30.73 & 35.19 \\
cluster26 & 20 & 3 & -82.003 & 37.44 & 44.94 \\
cluster27 & 23 & 3 & -80.958 & 25.75 & 33.89 \\
cluster28 & 26 & 3 & -79.921 & 20.20 & 19.54 \\
cluster29 & 27 & 3 & -79.083 & 30.96 & 27.68 \\
cluster30 & 31 & 2 & -75.267 & 19.35 & 30.27 \\
cluster31 & 30 & 1 & -76.274 & 27.37 & \\
cluster32 & 32 & 1 & -74.911 & & \\
\hline & & & & 25 \\
\hline
\end{tabular}

\section{References:}

(1) Li, S.; Duan, J.; Li, D.; Yang, B.; Dong, M.; Ye, K. Genes Dev. 2011, 25, 2409-2421.

(2) Guo, Q.; Yuan, Y.; Xu, Y.; Feng, B.; Liu, L.; Chen, K.; Sun, M.; Yang, Z.; Lei, J.; Gao, N. Proceedings of the National Academy of Sciences 2011, 108, 13100-13105.

(3) Yang, B.; Wu, Y. J.; Zhu, M.; Fan, S. B.; Lin, J.; Zhang, K.; Li, S.; Chi, H.; Li, Y. X.; Chen, H. F.; Luo, S. K.; Ding, Y. H.; Wang, L. H.; Hao, Z.; Xiu, L. Y.; Chen, S.; Ye, K.; He, S. M.; Dong, M. Q. Nat. Methods 2012, 9 , 904-906.

(4) Kahraman, A.; Herzog, F.; Leitner, A.; Rosenberger, G.; Aebersold, R.; Malmström, L. PLoS ONE 2013, 8, e73411.

(5) Schrodinger, LLC. 2010.

(6) Majorek, K. A.; Porebski, P. J.; Dayal, A.; Zimmerman, M. D.; Jablonska, K.; Stewart, A. J.; Chruszcz, M.; Minor, W. Mol. Immunol. 2012, 52, 174-182.

(7) St-Jean, M.; Lafrance-Vanasse, J.; Liotard, B.; Sygusch, J. J. Biol. Chem. 2005, 280, 27262-27270. 